\title{
Development of the Iron Electrode for Utility Scale Energy Storage
}

\author{
Barnes, Alistair D.
}

How to cite:

Barnes, Alistair D. (2019) Development of the Iron Electrode for Utility Scale Energy Storage. Doctoral thesis, Swansea University.

http://cronfa.swan.ac.uk/Record/cronfa51309

Use policy:

This item is brought to you by Swansea University. Any person downloading material is agreeing to abide by the terms of the repository licence: copies of full text items may be used or reproduced in any format or medium, without prior permission for personal research or study, educational or non-commercial purposes only. The copyright for any work remains with the original author unless otherwise specified. The full-text must not be sold in any format or medium without the formal permission of the copyright holder. Permission for multiple reproductions should be obtained from the original author.

Authors are personally responsible for adhering to copyright and publisher restrictions when uploading content to the repository.

Please link to the metadata record in the Swansea University repository, Cronfa (link given in the citation reference above.)

http://www.swansea.ac.uk/library/researchsupport/ris-support/ 


\title{
Development of the Iron Electrode for
}

\section{Utility Scale Energy Storage}

by

\author{
Alistair David Barnes
}

\author{
A thesis \\ submitted to Swansea University \\ in fulfilment of the \\ requirements for the degree of \\ Doctor of Engineering
}

College of Engineering, Swansea University, 2019

(C) Alistair Barnes, 2019 


\begin{abstract}
Iron electrodes, used in Nickel Iron $(\mathrm{NiFe})$ batteries, are known for their electrode stability, 25-year cycle life and suitability for utility scale energy storage. Key challenges addressed here include rapid electrode manufacture, analytical techniques and, cathodic hydrogen evolution.

Electrode manufacture was investigated in order to produce a low-cost iron electrode suitable for scale up. Ultrafast heating techniques, including near infrared, were used to produce electrodes on substrates with various surface modifications. Low temperature PTFE bound electrodes and sintered electrodes were produced via rapid heating. Electrochemical testing showed mixed results with sintered electrodes cycling successfully and no discernible effect from substrate surface modification.

The in-situ Scanning Vibrating Electrode Technique (SVET) has been used here for the first time in energy storage to study the parasitic hydrogen evolution reaction (HER) which occurs during charging and compromises round trip efficiency. SVET was used to map HER current density distribution across an iron electrode with respect to time and accuracy was verified against a traditional gas collection technique. Thiourea was shown to offer a $92 \%$ reduction in hydrogen evolution and the SVET was also shown to operate on sintered iron electrodes manufactured in earlier work.

The Scanning Kelvin Probe was used on iron electrodes to spatially resolve the Volta potential depression effect which occurs due to presence of atomic hydrogen. The mechanisms of HER inhibitors, benzotriazole and thiourea were compared against those described in literature. Differing mechanisms were confirmed, further demonstrating the potential of scanning electrochemistry in energy storage research.
\end{abstract}




\section{Author's Declaration}

This work has not previously been accepted in substance for any degree and is not being concurrently submitted in candidature for any degree.

Signed:

Date:

This thesis is the result of my own investigations, except where otherwise stated. Other sources are acknowledged by footnotes giving explicit references.

Signed:

Date:

I hereby give consent for my thesis, if accepted, to be available for photocopying and for inter-library loan and for the title and summary to be made available to outside organisations.

Signed:

Date: 


\section{Table of Contents}

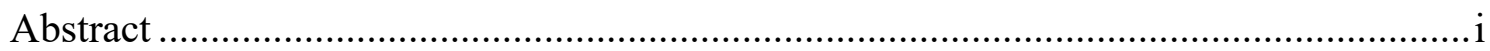

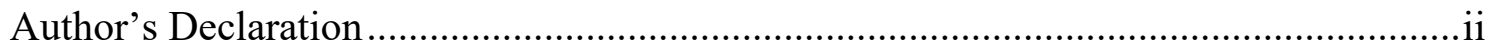

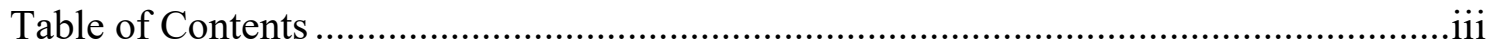

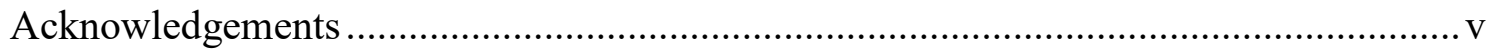

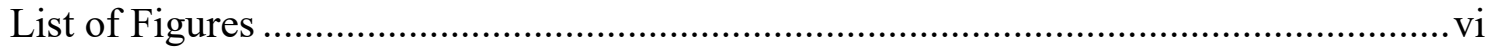

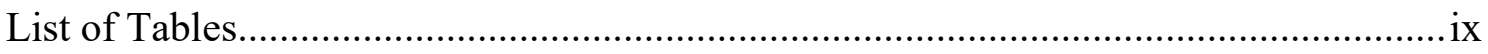

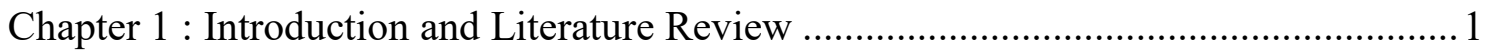

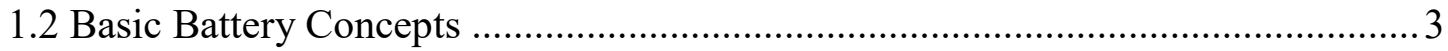

1.3 Commercially Available Secondary Battery Chemistries ..................................... 7

1.4 Key Research Areas in Secondary Battery Chemistries ..................................... 14

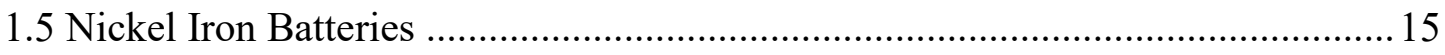

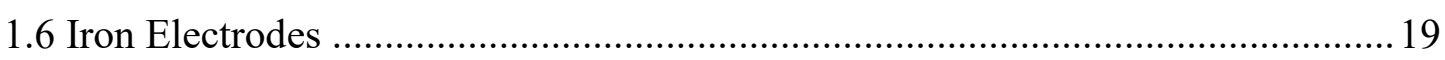

1.7 Scanning Electrochemistry for Energy Storage Research.................................2 27

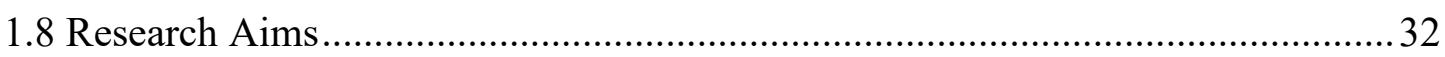

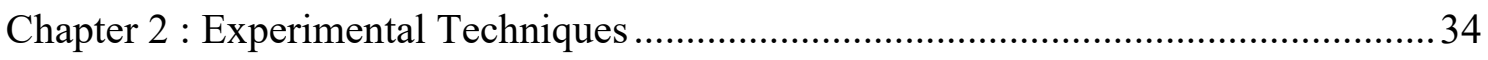

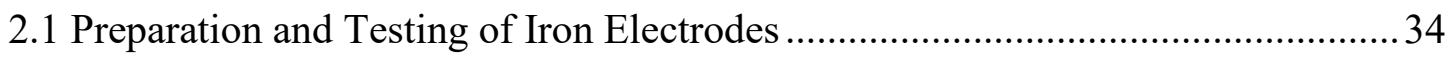

2.2 Scanning Vibrating Electrode Technique (SVET) for Investigation of Battery

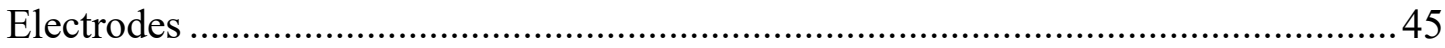

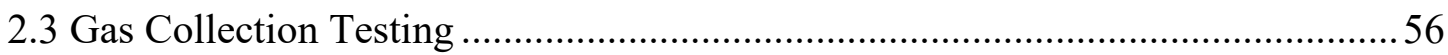

2.4 Scanning Kelvin Probe (SKP) for Iron Electrode Investigation ...........................59

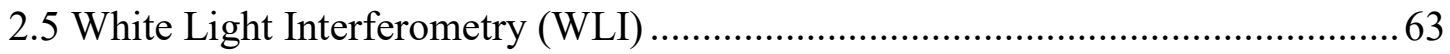

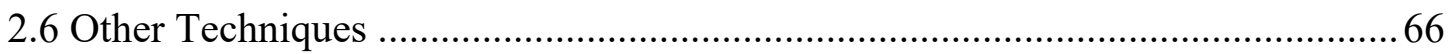

Chapter 3 : Printed Iron Electrodes for Low Cost Alkaline Batteries - Low Temperature

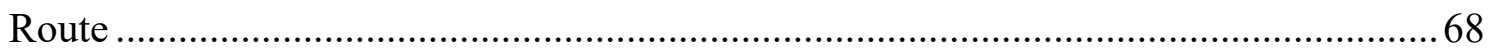

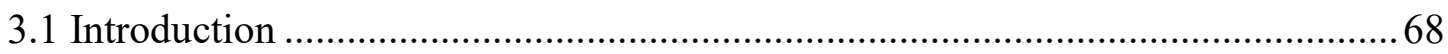

3.2 Preliminary Investigations: Low Temperature Route .................................... 70

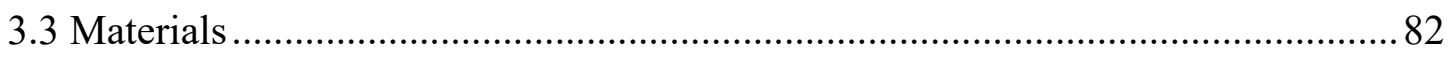

3.4 Rapid Low Temperature Electrode Manufacture Route ...................................... 83

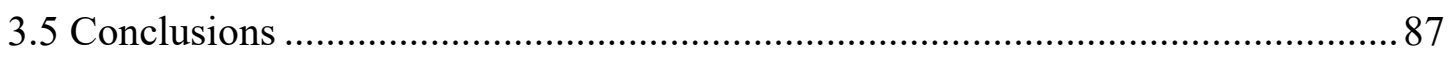

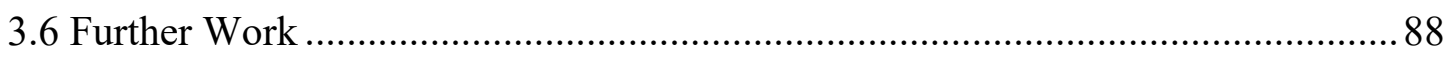


Chapter 4 : Printed Sintered Iron Electrodes for Low Cost Alkaline Batteries - High

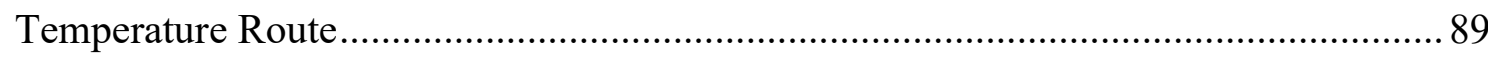

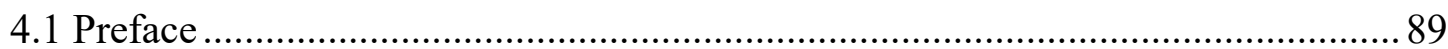

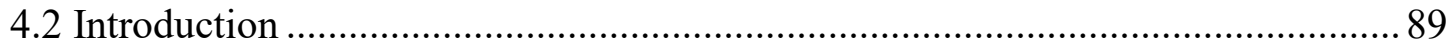

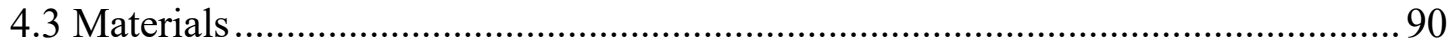

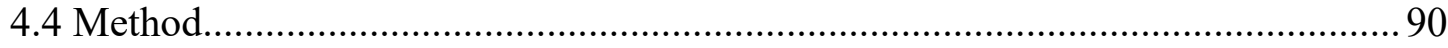

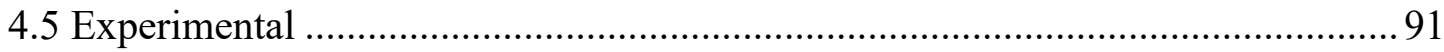

4.6 Sintered Electrodes for Electrochemical Testing ............................................. 111

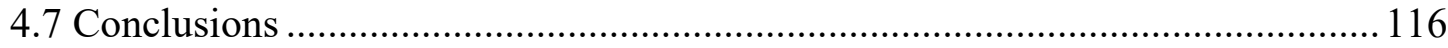

4.8 Blowing Agent Electrode for Further Investigation....................................... 117

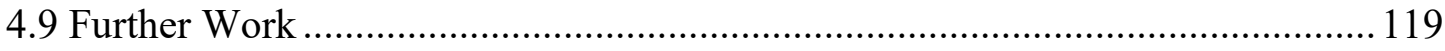

Chapter 5 : A Scanning Vibrating Electrode Technique for the Study of Hydrogen

Evolving from an Iron Electrode Battery.............................................................. 120

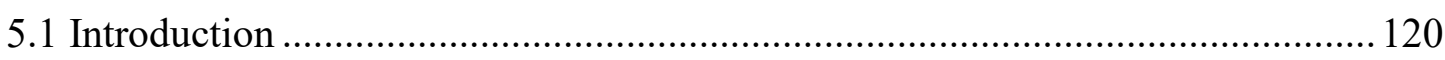

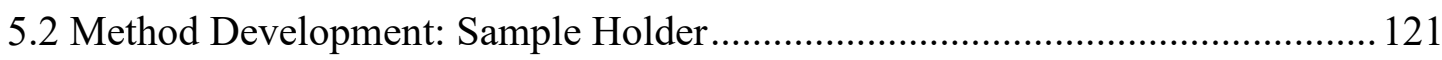

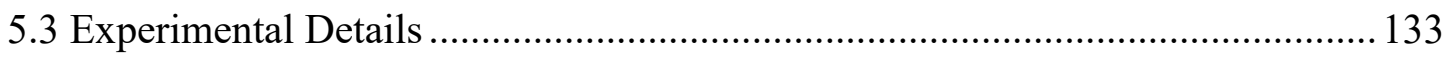

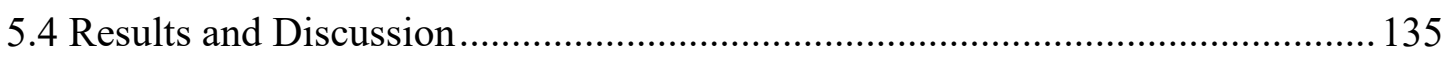

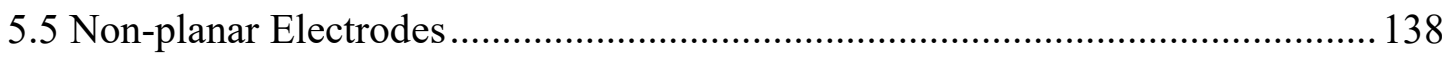

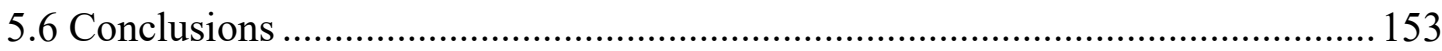

Chapter 6 : A Scanning Kelvin Probe Study of the Hydrogen Absorption on the Iron

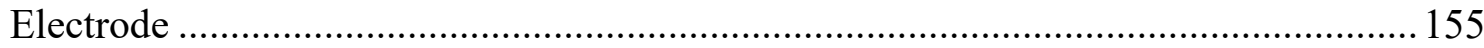

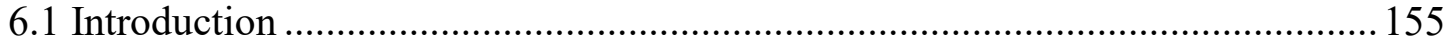

6.2 Ranking Performance of inhibitors using SVET .............................................. 156

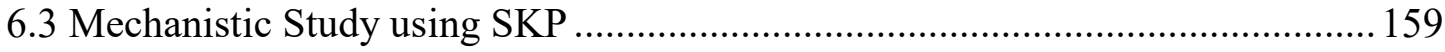

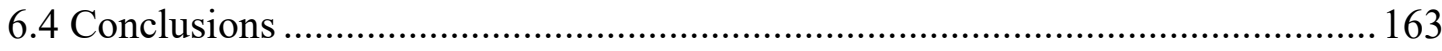

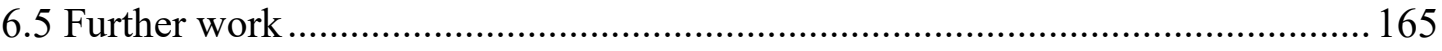

Chapter 7 : Conclusions and Further Work ….......................................................... 167

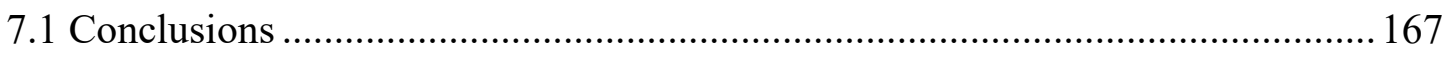

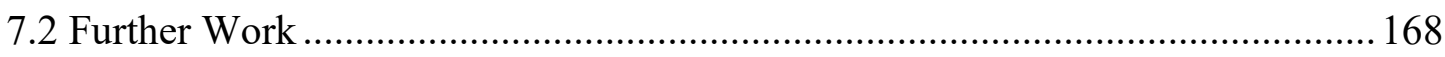

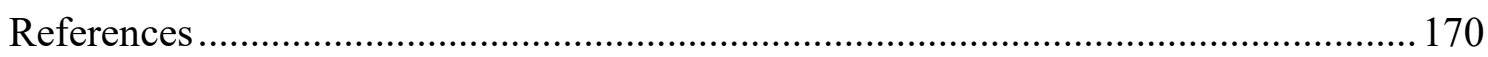

Appendix A Gwent Electronic Materials Ltd. Iron Inks.......................................... 185 


\section{Acknowledgements}

This has been a long journey and there are many people who are deserving of my appreciation for their extensive support. I've received unstinting supervision from Ian Mabbett, Jon Elvins, Jim Sullivan and Geraint Williams. I also wish to thank the batteries team, Carol, Jordan and Subbu, for their companionship and dedication.

I am proud to have been involved with SPECIFIC and the intelligent and talented people developing 'Buildings as Power Stations'. Assistance in the workshop from Lynsey, Stu, Leighton, Tom G and John D has been pivotal to the success of this project. I am also indebted to the expertise and generosity provided by Robin and Tina of Gwent Electronic Materials.

I have benefitted from the extensive and tireless coordination and support from those who make Swansea University's Doctoral Training Centres possible. Thank you. Swansea University staff and fellow students have helped extensively over the years. In particular, I appreciate the assistance, past and present, with various complex and temperamental equipment, educational and moral support, friendship, favours and the chore of travelling all the way to Hawaii with me. In order of increasing number of letters in their name and then alphabetically, thank you to: Amy, Ant, Des, Don, Jon, Nat, Ron, Sha, Tim, Tom D, Tom L, Alex, Dave S, Dave W, Khal, Kurt, Nath, Neil, Omar, Paul, Phil, Rhys andre, Chris, Becky B, Becky W, Bruce, Emily S, Emily R, James B, James E, Paddy, Steve, Calvin, Robbie and Margaret.

This work would not have been possible without funding from ESF, EPSRC, WAG and TATA Steel UK. These organisations have been generous enough to fund not only tuition, equipment and living expenses, but also to allow me to pursue my cycling interests. However, this has also necessitated further acknowledgement to the NHS fracture clinics for patching me up after various 'unintended excursions'. Also, 'shout out' to Hospital records for providing the soundtrack to this work.

Thanks also to my family, including both Dr Fanes, for the support. Sarah, thank you for your infinite patience, selflessness and endless encouragement. I wouldn't be me without you. Apologies, again, for forgetting to take you to Hawaii.

More recently, thank you to my colleagues at GKN Aerospace Additive Bristol for their understanding and motivation.

I have learnt a lot during these studies, some of it useful, but all of it valuable. I'm not sure if I'd do it again, but I certainly wouldn't have done it this time without those mentioned above and many others. Thank you all. 


\section{List of Figures}

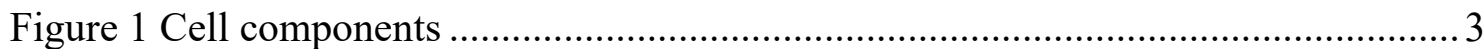

Figure 2 Electrochemical operation of a cell during charge[26] .................................. 4

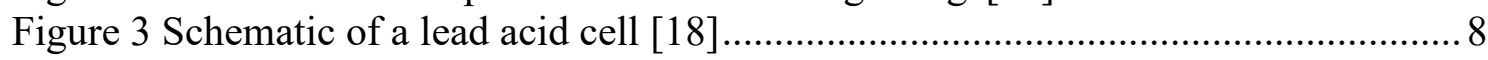

Figure 4 Sealed nickel cadmium cylindrical cell [26] .............................................. 10

Figure 5 Charge and Discharge reactions in a metal hydride electrode [34] .................. 11

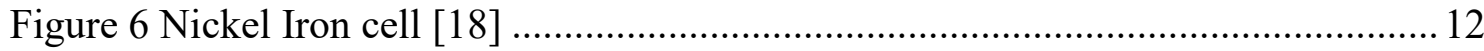

Figure 7 Lithium ion cell showing movment of ions during charge/dischage [34] ....... 13

Figure 8 SVET-derived image of corrosion activitiy [154] ........................................29

Figure 9 Scanning Kelvin Probe scan of a latent fingerprint [172] .............................. 30

Figure 10 Electron transfer reactions on the iron surface during hydrogen

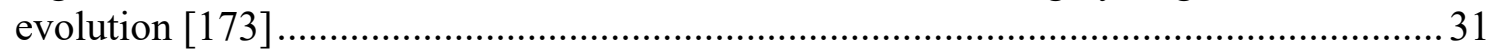

Figure 11 Jones Shipman 540 surface grinder fitted with magnetic bed chuck ............. 36

Figure 12 Flexovit grinding wheel above magnetic bed chuck .................................. 36

Figure 13 Adcock and Shipley manufactured series I milling machine ....................... 38

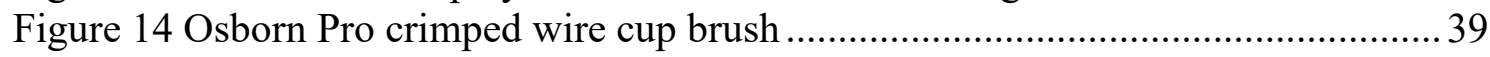

Figure 15 Example of tape casting using a glass rod ............................................... 40

Figure 16 NIR heating of iron-containing ink on a low carbon steel substrate .............. 42

Figure 17 Iwitani Rhesca Hot Dip Process Simulator. Furnace - left. Control system -

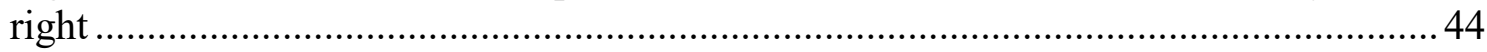

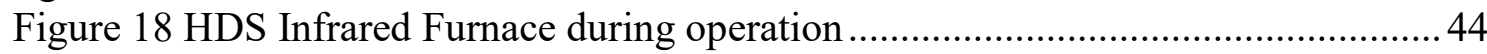

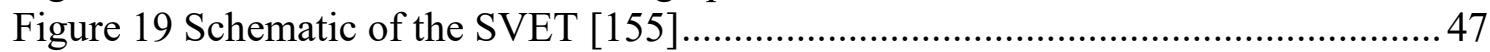

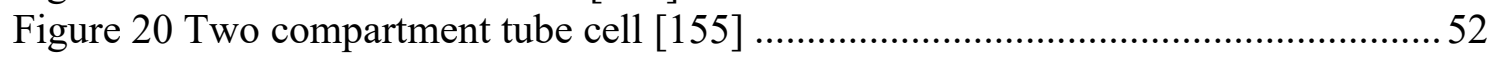

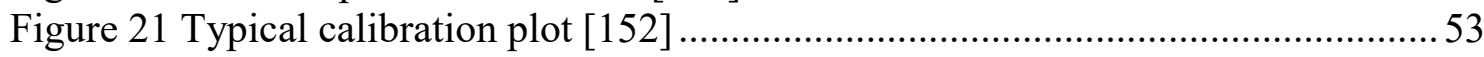

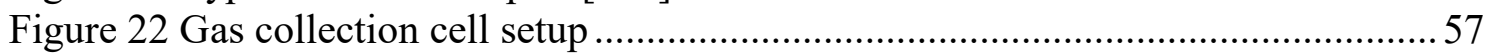

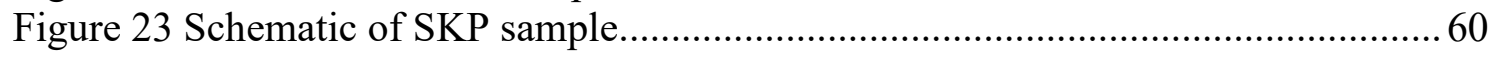

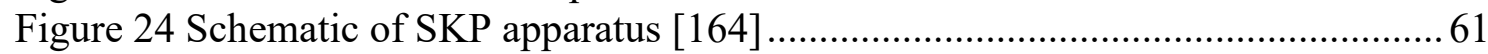

Figure 25 SKP-derived line scan across the reverse of the hydrogen evolution region .63

Figure 26 Schematic of a white light interferometry apparatus (adapted from [192]) ... 65

Figure 27 Results of 330 hour immersion test of coated iron and nickel substrates with

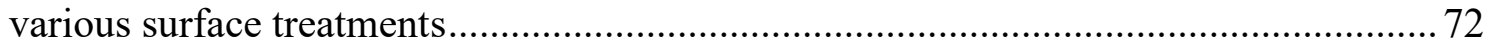

Figure 28 TGA of Gwent Electronic Materials Ltd. iron inks in air. Secondary axis is

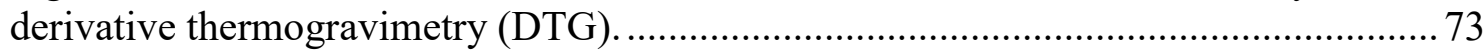

Figure 29 Preliminary test: C2150413D4 heated to $260{ }^{\circ} \mathrm{C}$ for 5 minutes .................... 76

Figure 30 EDS performed on iron containing ink in order to identify key phases ......... 77

Figure 31 Preliminary test: C2150413D4 Heated to $280{ }^{\circ} \mathrm{C}$ for 5 Minutes..................... 77

Figure $32 \mathrm{C} 2150413 \mathrm{D} 4$ Heated to $300^{\circ} \mathrm{C}$ for 5 Minutes ............................................ 78

Figure $33 \mathrm{C} 2150413 \mathrm{D} 4$ Heated to $320^{\circ} \mathrm{C}$ for 5 Minutes ............................................ 79

Figure 34 SEM of C2150413D4 Heated to $340{ }^{\circ} \mathrm{C}$ for 5 Minutes (initial hot plate

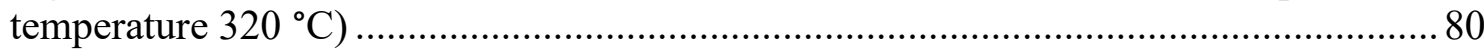

Figure 35 EDS of C2150413D4 heated to $340^{\circ} \mathrm{C}$ for 5 Minutes (initial hot plate

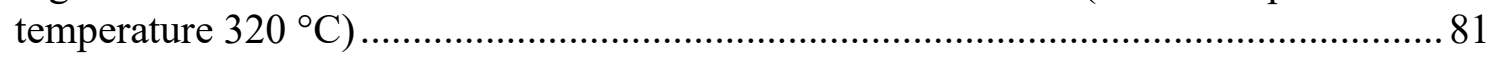

Figure 36 PTFE containing Iron ink sample heated on AdPhos NIR ............................ 86

Figure 37 Thermocouple data and HDS programmed set temperature from initial sintering conditions..... 
Figure 38 Initial sintering conditions sample (shown after sintering) .94

Figure 39 SEM image of sintered iron produced using initial sinter conditions 150x ...95 Figure 40 SEM image of sintered iron produced using initial sinter conditions 2000x . 96 Figure 41 SEM image of sintered iron produced using initial sinter conditions 4000x .97 Figure 42 Electrode with thicker layer of active material - evidence of ink running

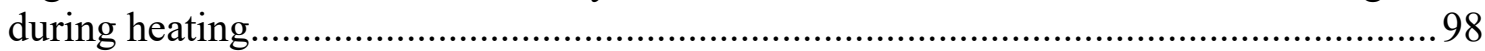

Figure 43 Sample sintered in HDS showing evidence of coating debonding ............... 100 Figure 44 SEM image of sintered iron which has partially debonded 30x ................... 101

Figure 45 SEM image of sintered iron which has partially debonded 250x ................. 102

Figure 46 SEM image of sintered iron which has partially debonded 500x .................. 103

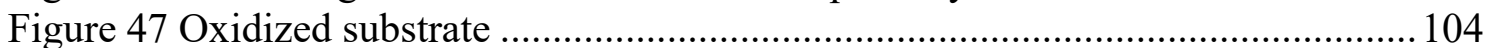

Figure 48 Oxidised pre-treatment after sintering in HDS ......................................... 105

Figure 49 Temperature log from HDS with reduced cooling rate ............................... 108

Figure 50 Sintered sample which had been cooled at a slower rate............................. 109

Figure 51 Iron coated samples sintered with increased cooling rate (nitrogen quench)

Figure 52 Average percentage utilization of sintered iron samples (error bars show standard error)

Figure 53 Charge and discharge capacity attained in cycling of sample 'Ground A'....114 Figure 54 Discharge/charge voltage profile vs. $\mathrm{Hg} / \mathrm{HgO}$ reference during

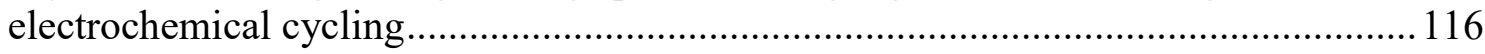

Figure 55 Various volume fractions of Expancel blowing agent added to iron ink ..... 118

Figure 56 Sintered iron using Expancel blowing agent ........................................... 119

Figure 57 Sample masked using Loctite Double Bubble Adhesive.............................. 123

Figure 58 Adhesive delamination as a result of hydrogen evolution........................... 123

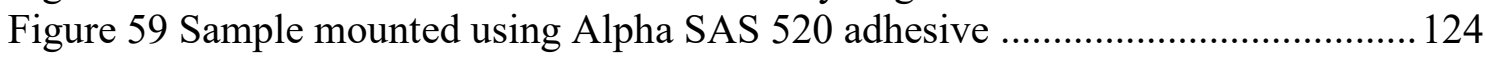

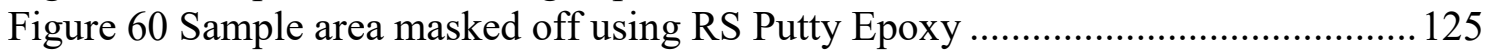

Figure 61 Top profile of sample holder showing shaded chamfer cut.........................126

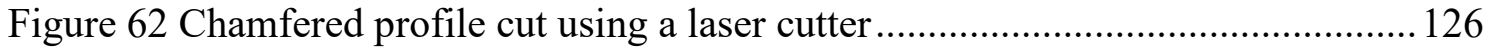

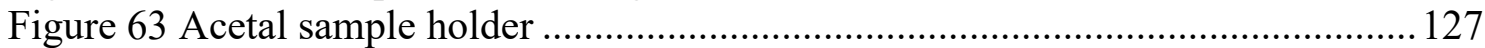

Figure 64 Rendered image of the designed sample holder ........................................ 128

Figure 65 Cross section drawing of the base of the sample holder............................. 128

Figure 66 Assembled sample holder ..................................................................... 129

Figure 67 EPDM gasket in underside of sample holder lid ......................................129

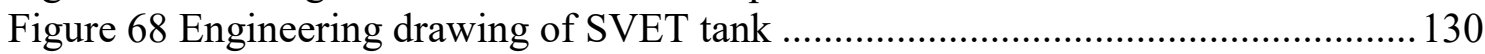

Figure 69 SVET tank with adjustable levelling stage and sample holder..................... 131

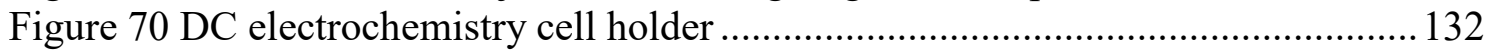

Figure 71 Completed sample holder and stage in Delrin ${ }^{\circledR}$ electrolyte tank ................ 133

Figure 72 Typical cyclic voltammograms for a solid iron electrode in $30 \% \mathrm{w} / \mathrm{v} \mathrm{KOH}$ electrolyte in the region in which hydrogen evolution occurs. With of i) thiourea, ii) sulfur, iii) lithium hydroxide and iv) no additions .................................................... 136 Figure 73 A typical SVET-derived current density surface map obtained by scanning an iron electrode surface when fully immersed in $30 \% \mathrm{w} / \mathrm{v} \mathrm{KOH}$ electrolyte, a) unpolarised and $b$ ), polarised to a potential of $-1.4 \mathrm{~V}$ vs. $\mathrm{Hg} / \mathrm{HgO}$ 137 Figure 74 a) Plots showing the amount of hydrogen evolved from an iron electrode charging potentiostatically at $-1.4 \mathrm{~V}$ vs. $\mathrm{Hg} / \mathrm{HgO}$ when fully immersed in $\mathrm{KOH}(30 \%$ 
w/v solution) measured using the SVET where i) no inhibitor, ii) $\mathrm{LiOH}$, iii) $\mathrm{S}$ and iv) Thiourea are present in the electrolyte. b) Plot showing the change in hydrogen evolution rate $\left(\Delta \mathrm{k}_{\mathrm{i}}\right)$ of hydrogen evolved measured using the SVET and the volumetric technique

Figure 75 Image of the SVET setup for the half ground sample

Figure 76 Detail view of experimentation showing apparent increased hydrogen evolution on ground area of sample....

Figure 77 WLI scan of as received surface of steel sample

Figure 78 Line scan showing measurement between a peak and a trough on as received

surface of steel sample

Figure 79 WLI scan of as ground surface of steel sample

Figure 80 Line scan showing measurement between a peak and a trough on ground surface of steel sample...

Figure 81 Height scan of half ground sample with annotation for the area which had been ground prior to height scan.

Figure 82 SVET (scan 1) of half ground substrate (ground area lowermost) ............... 145

Figure 83 Macro image of substrate with an area of sintered iron .............................. 146

Figure 84 WLI scan showing structure of sintered iron (left) on substrate (right) ....... 147

Figure 85 Cross section view of two sintered iron troughs......................................... 148

Figure 86 Wireframe representation of 3D height scan performed by SVET .............. 150

Figure 87 Current density as recorded by the 3D SVET, scan $1 \ldots \ldots \ldots \ldots \ldots \ldots \ldots \ldots \ldots \ldots . . . . . . . . . . . . .151$

Figure 88 Contour map showing current density as recorded by the 3D SVET (colored shading), with overlaid contour lines (grey solid lines) showing regions of lower height

as recorded by the height scan

Figure 89 3D wireframe with SVET-derived current density (scan 1) overlaid........... 153

Figure 90 Plot showing amount of hydrogen evolved from an iron electrode charging potentistatically at $-1.4 \mathrm{~V}$ vs. $\mathrm{Hg} / \mathrm{HgO}$ when fully immersed in $\mathrm{KOH}(30 \% \mathrm{w} / \mathrm{v})$ with inhibitors of thiourea $(0.1 \mathrm{M})$ and benzotriazole $(0.1 \mathrm{M})$

Figure 91 Cathodic current density maps from representative SVET scans. Left: $0.1 \mathrm{M}$ thiourea addition repeat $5,24^{\text {th }}$ scan (6 hours). Right: $0.1 \mathrm{M}$ thiourea addition repeat 7 , $9^{\text {th }} \operatorname{scan}(2.25$ hours $)$

Figure 92 Example 2d map from SKP-derived data of a sample post charging ........... 161 Figure 93 Volta potential drop vs. charging time resulting from hydrogen evolution on an iron susbstrate with a $0.2 \mathrm{M}$ potassium hydroxide electrolyte and addititions of benzotriazole and thiourea 


\section{List of Tables}

Table 1 Tata Steel DX54 low carbon steel chemical analysis, as reported via a personal comunciation to this authour 35

Table 2 Preliminary NIR Heating of 10\% PTFE Gwent Electronic Materials Fe Ink on $0.3 \mathrm{~mm}$ low carbon steel substrate. 85

Table 3 Utilization of sintered iron samples coated on to various substrates $\left(55^{\text {th }}\right.$ cycle) 


\section{Chapter 1: Introduction and Literature Review}

\subsubsection{Changes in Energy Generation}

The requirement for energy storage stems from the changes in the way electricity is generated. In the past, the majority of energy in the UK was generated from fossil fuels. This made storage simple; fossil fuels could be stored and electricity could be generated when it was required. UK demand could be forecast with high levels of accuracy and it could be ensured that supply would always meet demand.

The continual decrease in the cost of silicon photovoltaics is reducing the levelized cost of energy (LCOE) and subsequently, the need for a feed-in tariff to stimulate uptake of photovoltaic in the UK. Costs of crystalline silicon PV modules have fallen from over 75 \$/Watt [1] in 1977 to as low as 0.50 \$/Watt [2], according to research reported in the Elsevier journal, 'Solar Energy Materials \& Solar Cells'.

Germany, in particular, has invested heavily in photovoltaic and other renewable energy (RE) generation installations and is generally seen as a leader in RE in Europe. In 2017 it met more than $35 \%$ of its electricity generation needs from RE and at the start of 2018 the country was briefly powered entirely by RE [3], [4]. Total electricity generation from renewables became three times that of coal and Britain saw the first $24 \mathrm{~h}$ period without using any coal for electricity generation since the Industrial Revolution [5], [6].

Some authors say that as energy generation increasingly turns towards renewables, generation can only occur when environmental conditions are suitable [7]. Various models have been developed to show the effect of increased renewable energy generation on the stability of the electricity supply [8]-[11]. These authors argue that increased ratio of generation from unpredictable renewable energy technologies could lead to grid instabilities, as in certain conditions supply would not meet demand. However, Chauhan et al have proposed that certain artificial intelligence algorithms show promise in helping to overcome these challenges, albeit in stand-alone systems.

Integral to this transition is that industry and the residential sectors (henceforward, the 'utility scale') are gradually moving towards generating their own electricity with reduced reliance on large Power Stations. This author considerers large scale 'micro-generation' to represent a fundamental shift in energy generation and consumption. 
UK supermarket chain Sainsbury's was reported as being Europe's largest rooftop solar photovoltaic generator with installations with 170,000 panels split over 200 sites and a total generating capacity of $41 \mathrm{MWp}$ [12], [13]. It is reported on the website 'environment journal' that the UK now has around 800,000 domestic houses with solar photovoltaic installations, out of 27 million houses, however a source for this statement is not cited [14], [15].

The low financial reward on selling power to the grid may incentivize storage, given the correct technology. Specifically, Zheng et al state peak shaving is already viable with certain technologies within a US market [16]. Renewable Energy Storage is increasingly entering public awareness, with Nissan recently launching their Lithium Ion based storage offering [17]; however, safety, lifetime and cost are frequently raised as concerns with the current lithium based technology [18]-[21].

This technology need is a key driver for research in the field of energy storage.

\subsubsection{Characteristics of an Ideal Electrical Energy Storage System}

According to various sources, viable energy storage systems will have to meet the following requirements [18], [22], [23]:

1. Low installed cost $(<\$ 100 / \mathrm{kWh})$

2. Very long operating life of over 5000 cycles, preferably a 25 -year life time

3. High round trip efficiency $(>80 \%)$

4. Scalability to MWh sized systems

5. Safety

In particular, 100/kWh is seen by many as an 'inflection point' and described in 'biofuels digest' as a 'holy grail' [24], [25].

This thesis will focus on the understanding and development of technology which would enable these requirements. 


\subsection{Basic Battery Concepts}

A battery is an electrochemical device used for the storage of electrical energy in a chemical form and this thesis deals exclusively with rechargeable (secondary) batteries.

\subsubsection{Cell Components}

Electrochemical cells consist of several key constituents: positive electrodes (cathodes), negative electrodes (anodes), electrolyte (usually a liquid) and a means of physical separation of the two electrodes (Figure 1). In this thesis, the terms 'positive' and 'negative' will be used to describe electrodes, as opposed to 'cathode' and 'anode', to avoid confusion when discussing discharging and charging states.

Separators avoid electrical contact of the two electrodes, allowing transmission of ionic species only, thus ensuring the electrons must travel around the external circuit.

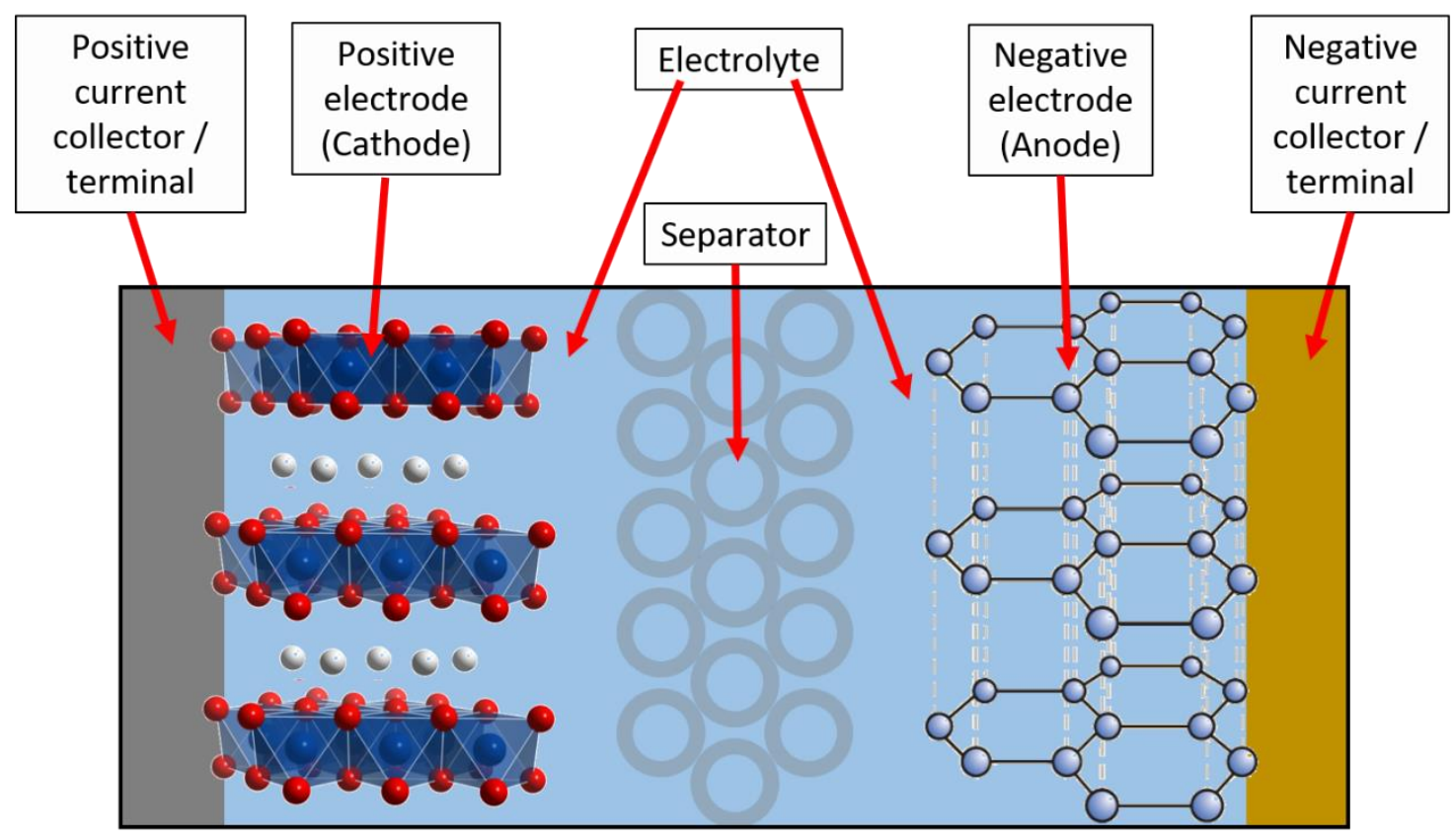

Figure 1 Cell components

\subsubsection{Operation of a Cell}

Cell discharge is initiated by connection of a cell to an external load. During discharge, the negative electrode is oxidized and the positive electrode is reduced. The reverse 
occurs during charge - the negative electrode is reduced and positive electrode is oxidized. To complete the circuit, ionic species will migrate between the positive and negative electrodes (Figure 2) [26].

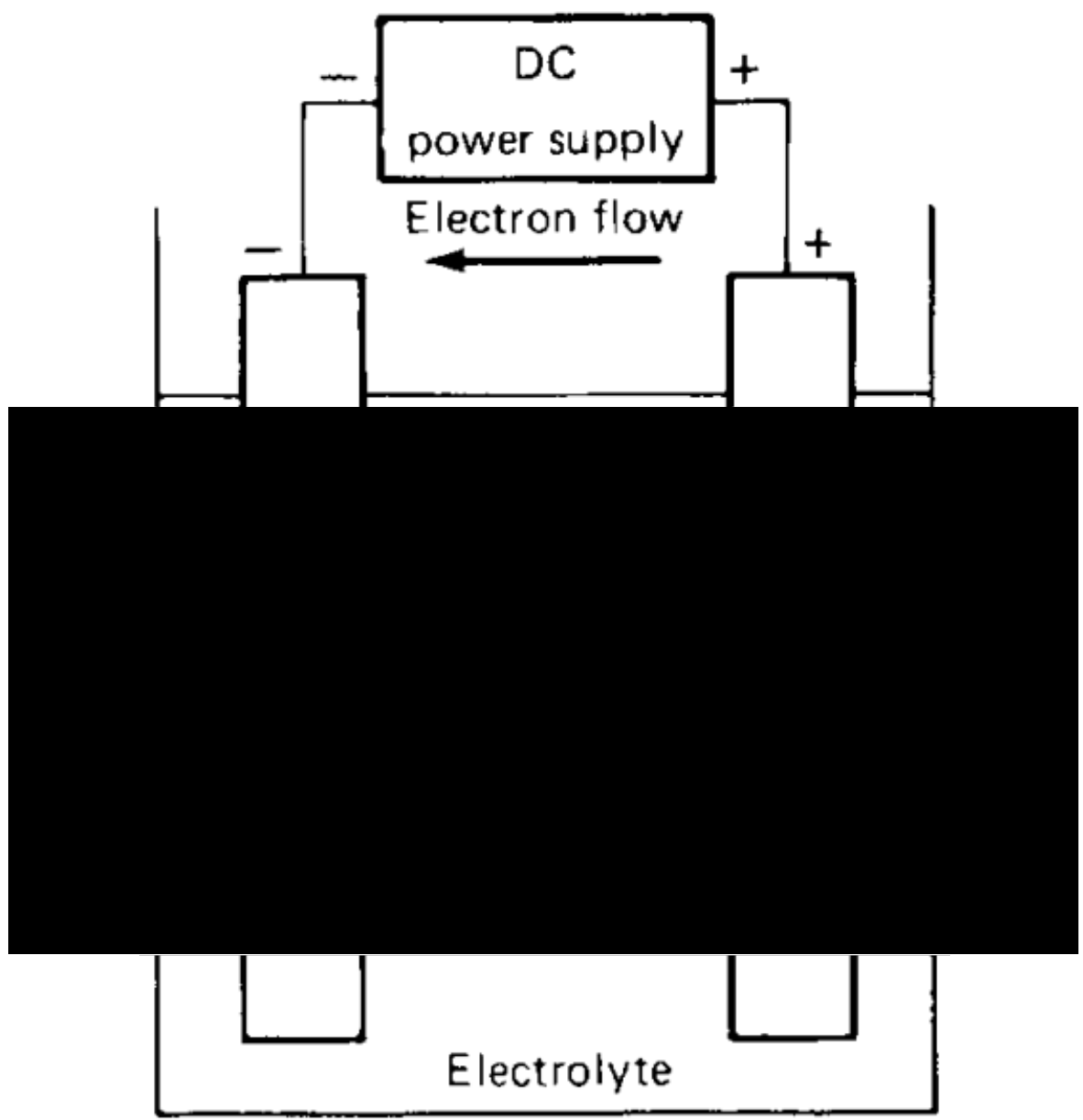

Figure 2 Electrochemical operation of a cell during charge[26]

Upon connection of an external power supply to a secondary cell, these reactions are effectively reversed and ions flow in the opposite direction to how they did during discharge. Galvanostatic or potentiostatic charging (or a combination) are chosen based on the chemistry of the cell. Charging endpoint detection also varies based on the chemistry in question. Some chemistries, such as lead acid, are typically 'float charged' to negate the effects of self-discharge (float charging is a constant low energy charge). Other cell chemistries may require particular charging regimes to reduce the chance of thermal runaway or extend cycle life [27]. 


\subsubsection{Definitions for Standard Terms}

\section{Theoretical cell voltage}

The theoretical cell voltage is a function of the materials which make up the positive and negative electrodes in a cell. Independently, the reactions occurring on both materials are termed 'half-cell reactions' and the combination of two half cells results in a complete cell. Accurate cell voltages can be determined experimentally, or calculations can be completed using the Nernst equation to account for non-equilibrium conditions; however an approximate value can be obtained using the standard oxidation and reduction potentials of the electrode materials, as shown in Equation 1.2-1 [26]:

$$
\begin{aligned}
& \text { Negative electrode material (oxidation potential) } \\
& + \text { positive electrode material (reduction potential) } \\
& \approx \text { standard cell potential }
\end{aligned}
$$

Using a Nickel Iron cell as an example to calculate theoretical open circuit voltage, the oxidation and reduction potentials are shown in Equations 1.2-2 and 1.2-3 (where $E^{0}$ is the electrode potential for the reduction reactions):

$$
\begin{array}{ll}
\mathrm{Fe}(\mathrm{OH})_{2}+2 e^{-} \leftrightarrow \mathrm{Fe}+2 \mathrm{OH}^{-} & E^{o}=-0.88 \mathrm{Vvs.SHE} \\
\mathrm{NiOOH}+\mathrm{H}_{2} \mathrm{O}+e^{-} \leftrightarrow \mathrm{Ni}(\mathrm{OH})_{2}+\mathrm{OH}^{-} & E^{o}=-0.49 \mathrm{Vvs.SHE}
\end{array}
$$

And combining these for the discharge reaction results in the Equation 1.2-4 theoretical cell voltage:

$$
-(-0.88 V)+0.49 V=1.37 V
$$

It should be noted that the nominal voltage (V) during discharge of a Nickel Iron cell is actually around $1.2 \mathrm{~V}$ [26]. Differences between the actual cell voltage and the theoretical cell voltage occur due to factors including internal resistance and the overpotential required for key reactions to occur. For example, in the above system solubility of reaction intermediaries result in a high overpotential [29]. 


\section{Theoretical capacity}

The theoretical capacity of a cell is related to the amount of active material in the cell which is able to react during discharge and thus the amount of electricity obtained from the active materials. Fundamentally this is based on the material properties of the anode and cathode. This is normally given in ampere hours (Ah) per gram (g), although volumetric calculations are possible. For a Nickel $(\mathrm{NiOOH})$ and Iron cell the calculation on discharge would be as per Equations 1.2-5 and 1.2-6 [26]:

$$
\begin{gathered}
\mathrm{Fe}+2 \mathrm{NiOOH}+2\left(\mathrm{H}_{2} \mathrm{O}\right) \rightarrow 2 \mathrm{Ni}(\mathrm{OH})_{2}+\mathrm{Fe}(\mathrm{OH})_{2} \\
1.04 \mathrm{~g} / \mathrm{Ah}+3.42 \mathrm{~g} / \mathrm{Ah}=4.46 \mathrm{~g} / \text { Ah or } 0.224 \mathrm{Ah} / \mathrm{g}
\end{gathered}
$$

However, this unrealistically assumes the complete oxidation/reduction of the electrode materials, therefore it is typical that the practical capacity of the cell is far less than the theoretical capacity. This is due to practical limitations such as availability of the active material, typically limited to the surface of the electrode only. For this reason, electrode structuring is important, as only the surface material will active and able to react, which is the reasoning behind a whole new area research in microbatteries [20], [30]. Good electrode design is fundamental not only to the capacity but also high rate discharge.

In principle, a more accurate theoretical capacity could be calculated by making a cell model based on assumptions (or experimental values) for electrode utilization and allowances for the separator and electrolyte which add mass without contributing to the capacity of a cell. This would result in a theoretical capacity similar to the actual or practical capacity.

\section{Cycle life and calendar life}

Life of a battery system is highly dependent on the design, discharge rate, charging conditions and depth of discharge. A cycle of a battery is a discharge and charge cycle and the batteries cycle life is said to end when capacity drops below $80 \%$ of the capacity at manufacture [26]. 


\section{Charge rates}

Charge rates (generally expressed as C-rates) are commonly used to describe galvanostatic charging and discharging in a way which normalizes against the battery capacity and therefore allows comparisons between different sized cells and chemistries. A C rate of $\mathrm{C} / 5$ (also written as $0.2 \mathrm{C}$ ) would discharge a 1 Ah cell over five hours at 0.2 Amps (200 mA) and likewise, the cell could be fully recharged in five hours if provided with 0.2 Amps [31].

Whilst C-rates are common place in literature, work by Linden suggests that they can be confusion as they are dimensionally incorrect in terms of units [26].

\subsection{Commercially Available Secondary Battery Chemistries}

Lead acid and lithium ion rechargeable batteries make up the majority of the secondary battery market.

Many secondary batteries use an aqueous alkaline electrolyte such as potassium hydroxide or sulfuric acid. As they are water based, despite their strong $\mathrm{pH}$, they are popular due the safety advantages of being non-flammable. Acid electrolytes, such as the sulfuric acid used in lead acid batteries are prone to reacting with the electrode materials, which can be detrimental to the cycle life of the battery. Conversely, non-aqueous batteries are typically highly flammable, whilst they generally offer better performance than the aqueous alternatives.

Generally, this work focuses on aqueous batteries due to their inherent safety advantages. Non-aqueous chemistries are also considered for the purpose of context but more so because there are interesting technical aspects which may have application in aqueous systems.

Here, secondary chemistries which are widely commercially available are discussed. Promising research areas are discussed later in 1.4. 


\subsubsection{Lead Acid Batteries (PBA)}

Lead Acid Batteries (Figure 3) are the oldest secondary batteries, invented by Gaston Planté in 1859 [26]. They use lead and lead oxide electrodes which, upon discharge, both combine with the sulfuric acid electrolyte to form lead sulfate. There has been significant technical development allowing for production of sealed or valve regulated cells capable of starting internal combustion engines and use in stationary applications such as UPS (uninterruptable power systems) and backup power supplies. Recombination catalysts encourage oxygen recombination to the extent that gas emissions are decreased by $95 \%$.

Lead acid batteries are considered to be a mature technology [18]. They are characterized by Low cost, low self-discharge, low specific energy, good high rate discharge and good low temperature performance. Dendrite formation is a key challenge. This technology is not of research interest due to its tendency to form lifetime limiting dendrites. Despite the above-mentioned technical and commercial maturity of the technology, dendrite formation has not been eliminated. This makes it a poor fit for utility scale energy storage.

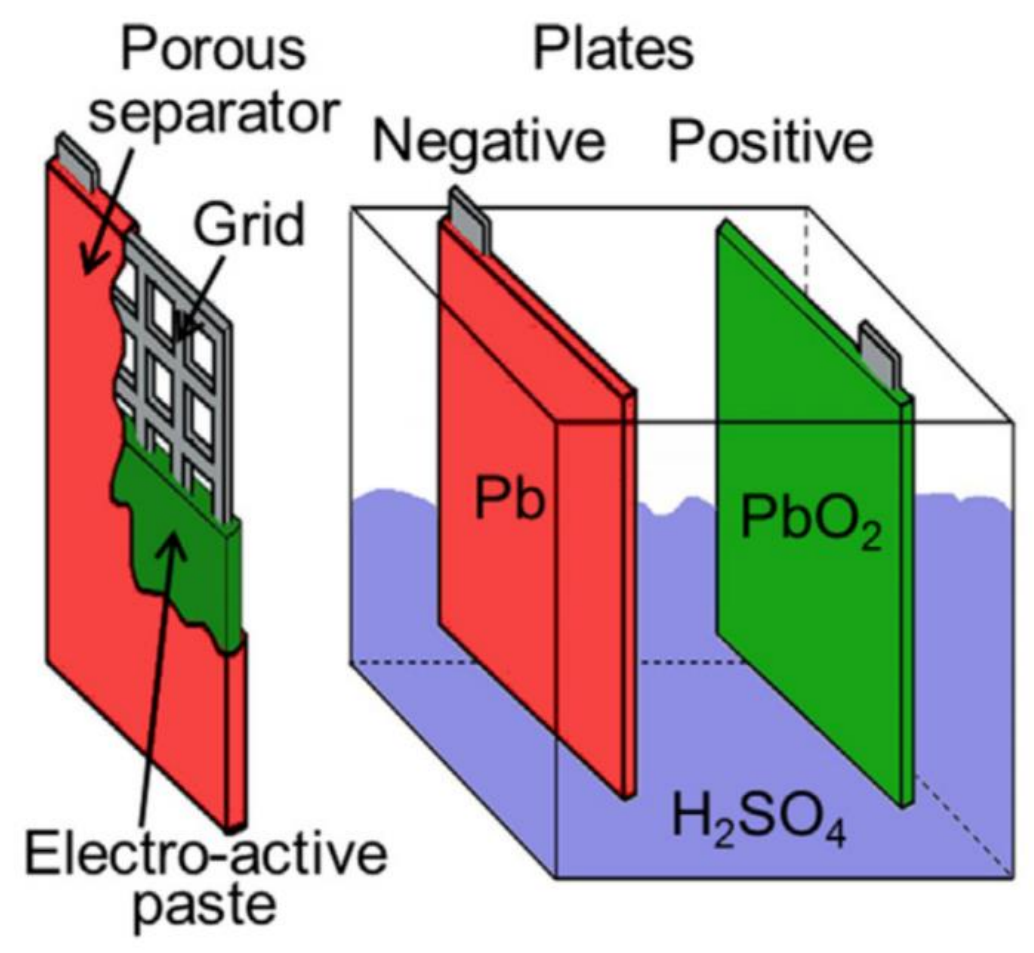

Figure 3 Schematic of a lead acid cell [18] 


\subsubsection{Nickel Cadmium Batteries (NiCd)}

Whilst cadmium is well known to be an environmental hazard and a health hazard, NiCd batteries have been produced for over 30 years in several guises such as pocket plate, sintered plate and vented and sealed (Figure 4) with an oxygen recombination catalyst [32]. Their operation depends on the metallic cadmium electrode combining with hydroxide anions from the potassium hydroxide electrolyte and the nickel electrode being reduced. They are well regarded for their rate capability, although recent research efforts have been to improve energy density. A memory effect can occur during repeated partial discharge of the battery leading to reduced capacity. NiCd cells are rugged and have a long cycle life, allowing them to be used in heavy duty industrial applications [18]. Despite this, research in this area is not considered as the environmental impacts of the cadmium electrode have resulted in prohibition of the sale of $\mathrm{NiCd}$ batteries expect for legacy applications where no alternative is available [33]. 


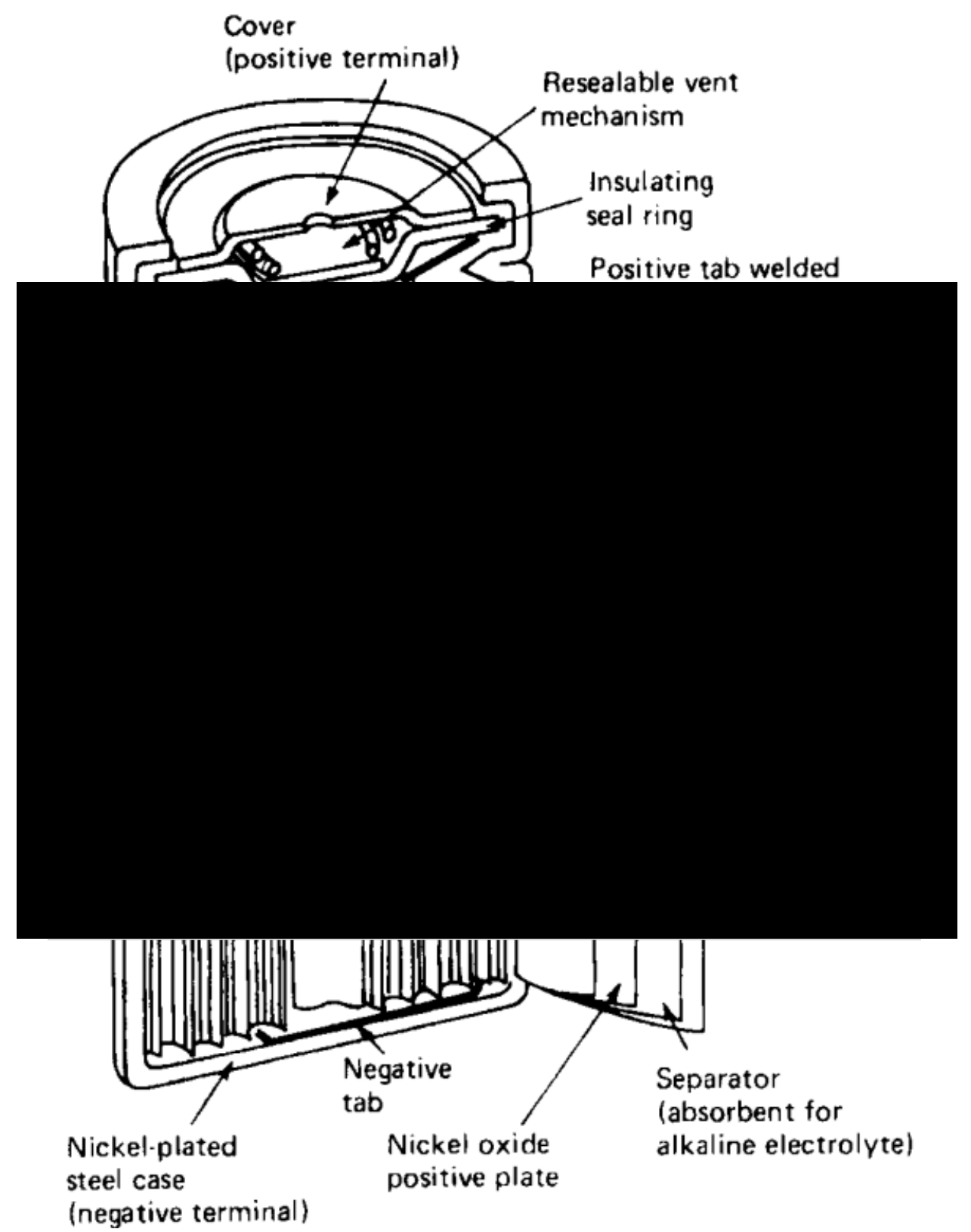

Figure 4 Sealed nickel cadmium cylindrical cell [26]

\subsubsection{Nickel-metal Hydride Batteries (NiMH)}

Nickel-metal hydride batteries represent a somewhat more environmentally friendly alternative to $\mathrm{NiCd}$ batteries with higher energy density and reduced cycling ability [18]. The cadmium electrode in 1.3.2 is replaced by a metal hydride electrode (Figure 5) which acts to absorb hydrogen upon on charge. The nickel electrode operates in the same principle as in NiCd (1.3.2). NiMH batteries are considered to be safe and tolerant to 
abuse and mishandling when compared with technologies such as $\mathrm{NiCd}$ and Lithium Ion. These have been used in hybrid electric vehicles for regenerative breaking energy storage and are commonly used in small domestic rechargeable batteries. High charge retention performance of $85 \%$ over 1 year is an important feature for the domestic markets but has less significance for diurnal utility scale energy storage systems. Also prone to memory effect [26]. The relatively poor cycle life vs nickel iron batteries and the frequent usage of rare earth materials in the hydride electrodes make this an unfavorable technology to consider for research.

Charge

Discharge

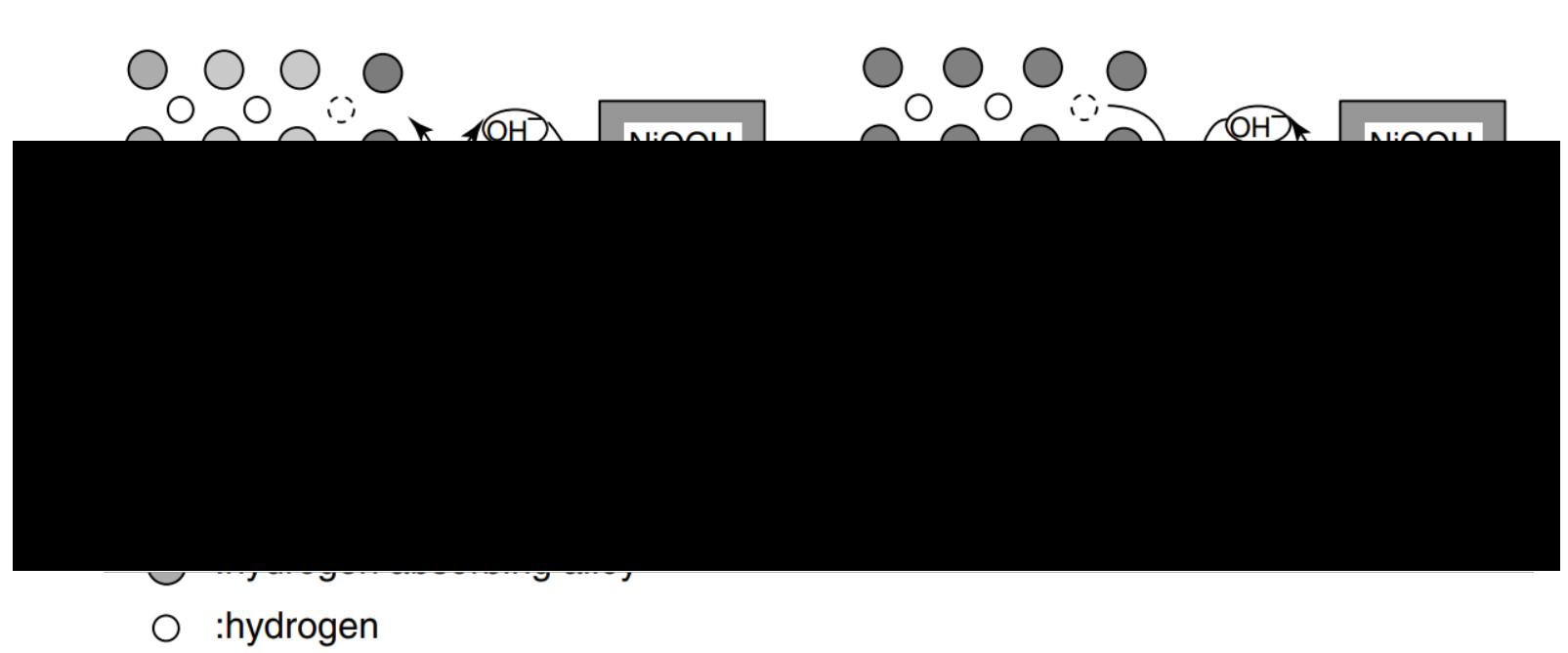

Figure 5 Charge and Discharge reactions in a metal hydride electrode [34]

\subsubsection{Nickel Iron Batteries (NiFe)}

Nickel Iron technology was first researched by Waldemar Jungner in 1899 and later commercialized by Thomas Edison who claimed that the design was 'far superior to batteries using lead plates and acid' [32], [35]. They use a metallic iron electrode combined with the previously discussed nickel hydroxide counter electrode (1.3.2 and 1.3.3) and a potassium hydroxide electrolyte (Figure 6).

Nickel Iron batteries are famed for their durability and long life but were largely superseded by lead acid batteries on the grounds of cost, maintenance requirements and specific energy [36]. NiFe chemistry has an extremely long life (20+ years). Original batteries produced $50+$ years ago by the Edison Storage Battery Company are generally 
found still to be working even after long periods of dormancy. Cycle life is decreased by frequent rapid discharge (high current) operations - although this problem has been combatted in other batteries by use of sintered electrodes [37]. A hydrogen evolution reaction upon charge decreases the round trip efficiency and results in consumption of the electrolyte which then requires replacement [26].

On the basis of the relative immaturity of the nickel iron battery combined with the long cycle life, this technology is a key research area in this thesis. The chemistry as a whole is discussed in 1.5 and aspects specific to the iron electrode are discussed in 1.6.

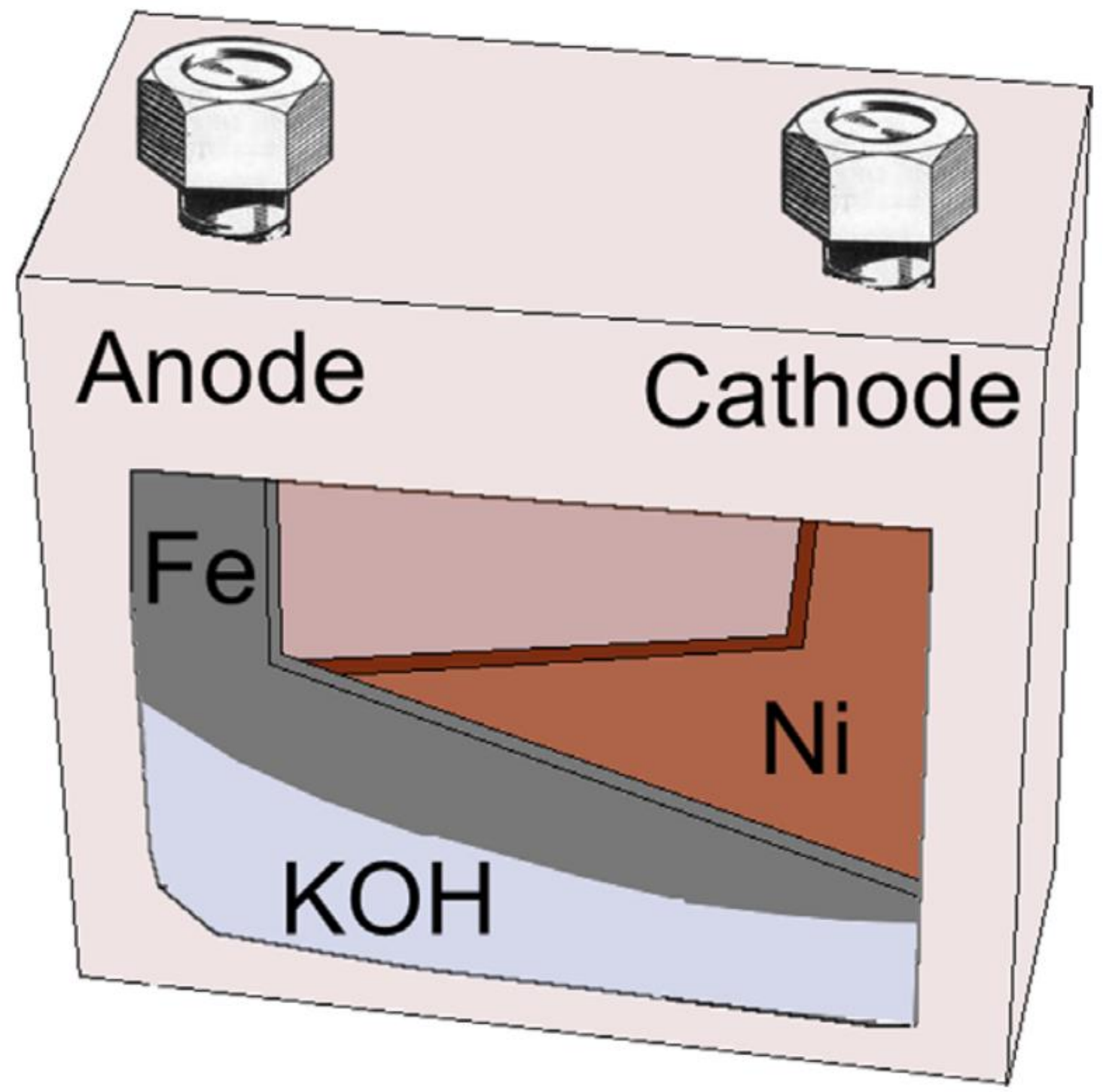

Figure 6 Nickel Iron cell [18] 


\subsubsection{Lithium lon Batteries (LIB)}

The rapid uptake in portable electronics such as mobile phones and laptops has led to major research and investment in lithium ion batteries as they offer an increased energy density and specific energy compared to previous portable/sealed technologies, mainly $\mathrm{NiCd}$ and NiMH. There has been significant research attention and consumer uptake of LIB's, which is technically a family of chemistries with minor variations in electrode and electrolyte compositions. Production is reported to have exceed 250 million cells per month and lithium ion production demand is expected to exceed $120 \mathrm{GWh}$ in 2020 [38], [39].

Battery management circuitry is required to prevent over charge and over discharge. Safety is a concern as thermal runaway can result in rapid and explosive fires due to electrode and decomposition and flammable electrolytes [21]. Lithium ion batteries operate on the principle of intercalation (Figure 7), where they typically shuttle between a graphite negative electrode and a lithium ion receptive positive electrode. Lithium salts are dissolved in an organic electrolyte. It is the flammable electrolyte which results in this typical lithium ion chemistries being discounted from further research, this author considers this property incompatible with utility scale energy storage.

\section{Charge}

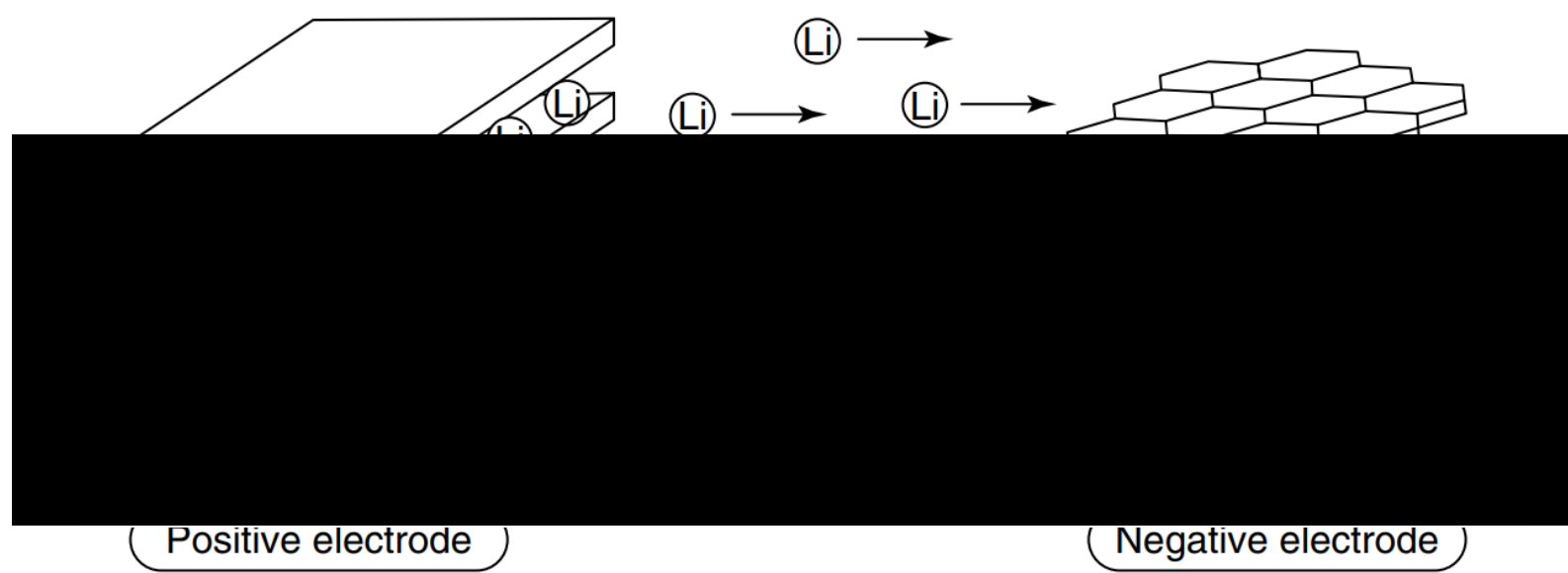

Figure 7 Lithium ion cell showing movment of ions during charge/dischage [34] 


\subsection{Key Research Areas in Secondary Battery Chemistries}

In this section, various research areas of particular interest to this author are discussed. Whereas section 1.3 focusses on commercially available systems, the chemistries discussed in this section are typically in the research or early commercialization stages.

These chemistries are chosen to provide context and an overview of some of the current key research areas in secondary batteries. This author acknowledges that there are many other topics which could be discussed and the topics below could be discussed in more detail, but this is considered to be out of the scope of this thesis.

\subsubsection{Sodium Ion Batteries (SIB)}

Sodium ion batteries are seen as a promising alternative to LIBs, primarily due to reduced relative cost of sodium salts and concerns over long term availability of lithium [18]. However, there are multiple fundamental issues preventing widespread adoption at the time of writing. 'Aquion Energy' are notable due to their commercialization of an ‘Aqueous Hybrid Ion' chemistry which utilizes sodium ions along with $\mathrm{Li}^{+}$and $\mathrm{H}^{+}$[40], [41]. Despite the similarity of $\mathrm{Li}^{+}$and $\mathrm{Na}^{+}$, electrode materials which are ideal for the former are rarely suitable for the latter. Identification of $\mathrm{Na}^{+}$compatible electrode materials still poses a challenge, in part due to volume changes [42], [43]. The majority of research papers reviewed focus on identifying suitable electrode materials. There are also efforts to produce solid state SIB's [44], [45]. The desire of this work is to focus on research to improve an existing technology and accordingly, SIBs are not investigated further.

\subsubsection{Solid State Lithium Ion and Sodium Ion}

Dendrite formation is a common issue in energy storage [18], [26]. Systems where dendrite formation does not occur could offer longer lifetimes and significantly higher $\mathrm{C}$ rates. Therefore, work published by M. Braga, J. Goodenough and others detailing development of a solid glass electrolyte suitable for use with $\mathrm{Li}^{+}$(and also $\mathrm{Na}^{+}$) was well received [46], [47]. This is a notable advance, as solid state systems do not form dendrites [48]. John Goodenough is famed for his work in development of LIB's in the 
1970's/1980's but admits that liquid electrolytes cannot offer the performance required and alternatives are needed [49]. Electric car company 'Fisker Inc' has stated intentions to produce electric cars using solid state batteries [50], [51]. This work is in relatively early stages and yet to be proven to be cost effective and delivered commercially. Fundamental research of this nature is outside of the scope of this research which intends to progress technologies which are already commercially available.

\subsubsection{Metal Air Batteries}

Air electrodes typically employ a metal foil negative electrode and an external positive electrode which functions using oxygen in ambient air [18]. The potential for an exceptionally high energy density battery drives this research, although FigueredoRodríguez et al note that air electrodes still have complex challenges associated with them [29]. Metal air cells with a variety of negative electrodes are possible: $\mathrm{Li}, \mathrm{Ca}, \mathrm{Mg}, \mathrm{Al}, \mathrm{Zn}$ and $\mathrm{Fe}$, some of which suffer from dendrite formation which could potentially limit cell lifetime [29]. These can use either a third electrode or a bifunctional electrode to sustain both oxygen reduction and evolution [26]. Iron air cells offer several advantages over other metal air chemistries due to potential for low cost, long cycle life and freedom from dendrites due to insolubility of the reaction intermediates in the electrolyte [29], [52], [53]. Whilst typically, it is the air electrode which requires further development to make a metal air system viable, development of a negative electrode, such as the iron electrode, would improve the future performance of a metal air chemistry. Accordingly, the potential for the iron electrode research discussed in this thesis to be utilized for iron air batteries should not be discounted.

\subsection{Nickel Iron Batteries}

A brief introduction to NiFe batteries was given in 1.3.4. This chapter aims to further explore the nickel iron chemistry and its potential for use in utility scale energy storage. Nickel iron batteries are chosen for further attention, not because they are the highest performing of all chemistries, save for a promising cycle lives, but because they have received relatively little research attention and provide many interesting areas of research. In this section the historical context of the chemistry is discussed, along with aspects of 
the cell chemistry which are specific to the cell as a whole - detail specific to the iron electrode is contained in 1.6.

Nickel Iron batteries are still produced commercially by Sichuan Changhong Battery Co., Ltd using somewhat outdated technology gained from VARTA [55]. Many of these cells are rebranded and sold through various international distributors for use as off grid storage. They are marketed as a moderately clean technology when compared to lead acid batteries and lack of flammability is an advantage against technologies which frequently use organic electrolytes, such as lithium ion. Additionally, it is well known that Nickel Iron batteries are extremely resilient to mistreatment, by over charge, over discharge and long periods of dormancy [20], [26], [37], [54].

One study reported by Mabbett et al show the potential for significant improvements to the technology by updating the manufacturing routes used to produce the electrodes [37]. The majority of authors reviewed were confident that there was significant scope for improvement to the technology which would increases its suitability for utility scale energy storage applications.

\subsubsection{History of Literature}

Since 'falling out of favor' to the more cost effective and power dense lead acid batteries, nickel iron has received limited industrial and academic attention in comparison to other technologies, such that it is worth a brief discussion for a historical perspective into this author's research [18].

Salkind, through his interest in portable medical devices, authored 'Alkaline storage batteries,' pioneered several techniques and was awarded several patents [56]-[60]. There was active research on $\mathrm{NiFe}$ and iron air batteriesin the late 1970s by Öjefors et al. The work in 1978 was with the Swedish National Development Co. and included manufacture and testing of a $30 \mathrm{kWh}$ Iron air cell [53], [61], [62]. Westinghouse Corporation also worked on this technology in the same period [63]-[65].

Shukla et al have been prolific researchers in this field since 1980s up to the present day, with much of this work focusing on mechanisms of additives [28], [66]-[74]. The iron electrode has also been studied extensively by Periasamy et al [75]-[78]. 
More recently, Narayanan et al have published extensively on various production methods of iron electrodes and additives for improving their performance in work primarily published in the Journal of the Electrochemical Society and funded by the U.S. Department of Energy [19], [22], [52], [79]-[85]. In this same time period, Posada and Hall et al of the University of Sheffield have written several articles on iron electrode additives [54], [86]-[91] and have completed a review on aqueous batteries with contribution from this author [18].

Further commercialization has been attempted in recent years by Encell Technology Inc. various patents have been awarded but exact details on scientific advances have not been published [92]-[99].

\subsubsection{NiFe Cell Chemistry}

Nickel electrodes are produced in a large scale and are well understood due to the years of research that has gone into $\mathrm{NiCd}$ and $\mathrm{NiMH}$ technologies. The nickel electrode will not be discussed in detail as it is outside the scope of this thesis.

The iron electrode is less understood and has received less attention as it is not currently used in any mass-produced batteries.

Nickel Iron cells consist of iron electrodes physically separated from nickel oxyhydroxide electrodes. The overall reaction consists of oxygen being transferred from one electrode to the other. This reaction is complex and is known to include many transitional species [59], [61], [100].

The overall reaction in a nickel iron cell can be written as per Equations 1.5-1 and 1.5-2:

$$
\begin{array}{cc}
\mathrm{Fe}+2 \mathrm{NiOOH}+2\left(\mathrm{H}_{2} \mathrm{O}\right) \rightleftharpoons 2 \mathrm{Ni}(\mathrm{OH})_{2}+\mathrm{Fe}(\mathrm{OH})_{2} & \text { (First plateau) } \\
3 \mathrm{Fe}(\mathrm{OH})_{2}+2 \mathrm{NiOOH} \rightleftharpoons 2 \mathrm{Ni}(\mathrm{OH})_{2}+\mathrm{Fe}_{3} \mathrm{O}_{4}+2 \mathrm{H}_{2} \mathrm{O} \quad \text { (Second plateau) }
\end{array}
$$

Note: the discharge reaction is read left to right.

\section{Iron electrode reactions}

The reactions of the negative iron electrode can be seen in Equations 1.5-3 to 1.5-5. The plateaus are present due to the $+2,+3$ oxidation states of the iron. 


$$
\mathrm{Fe}+\mathrm{nOH}^{-} \rightleftharpoons \mathrm{Fe}(\mathrm{OH})_{n}^{(2-n)}+2 e^{-} \quad E^{0}=-0.877 V \text { vs. SHE }
$$

And under deep discharge when electrodes are arranged in a negative limited

$$
\begin{aligned}
& \text { configuration: } \\
& \mathrm{Fe}(\mathrm{OH})_{2}+\mathrm{OH}^{-} \rightleftharpoons \mathrm{Fe}(\mathrm{OH})_{3}+e^{-} \\
& \text {Or: } \\
& \mathrm{Fe}(\mathrm{OH})_{2}+\mathrm{OH}^{-} \rightleftharpoons \mathrm{FeOOH}+\mathrm{H}_{2} \mathrm{O}+e^{-} \quad\left(\mathrm{E}^{0}=-0.560 \mathrm{~V} \text { vs. SHE }\right) \quad \mathbf{1 . 5 - 5}
\end{aligned}
$$

Where the electrode potential $\left(\mathrm{E}^{0}\right)$ for each oxidation reaction is shown with reference to the standard hydrogen electrode (SHE).

\section{Cycle life}

In most chemistries, cycle life varies considerably with depth of discharge and discharge rates, this effect is less pronounced in NiFe [83].

Solubility of the electrode materials and derivative compounds is typically a factor affecting the cycle life of a cell. NiFe cells are capable of extreme cycle lives due to the low solubility of iron and nickel and their hydroxides in the alkaline electrolyte. It has been reported that nickel iron cells still function well after exceeding the cycle life of most other competing technologies three times over [56], [62], [63], [101].

\section{Capacity and power}

Heavy electrode materials and poor utilization and coulombic efficiencies result in poor specific and volumetric capacities and power capability for NiFe cells, particularly when compared with energy-dense systems such as LIBs (1.3.5).

'Nanostructured' electrodes have been researched due to potential for higher power as well as increased utilization resulting in increased capacity [20], [29], [102]-[104]. Layered electrodes have been developed in other chemistries [105]-[107], but not seen in academic literature other than a patent by Encell Technology Inc. [96]. 


\section{Safety}

Whilst aqueous batteries generally are considered to be safe [18], the majority of publications reviewed contained positive comments from the authors regarding the safety of NiFe cells. In part, this is due to use of non-flammable materials, but the environmental credentials of the electrode materials are also often praised - although this is less true of $\mathrm{NiOOH}$ electrodes. This, combined with an exceptional cycle life, appears to be a strong motivation for research. Posada et al also make note of safety implications of hydrogen evolution reaction (HER) upon charge [86], however this author considers the inclusions of this criticism to be an angle for publication as opposed a valid concern due to a lack of focused analysis in the publication. This hydrogen evolution reaction (HER) is complex, with several alternative and well researched mechanisms described by the VolmerHeyrovsky-Tafel model [108]-[110]. As the electrolyte is consumed in the reaction, there is a maintenance requirement needed to replenish the electrolyte in equal amounts to in which it is consumed.

\subsection{Iron Electrodes}

\subsubsection{Introduction}

In this section, iron will be discussed as a negative electrode material for energy storage and gaps in literature will be identified and considered. Less focus will be placed on the chemistry of cells, such as nickel iron or iron air, as this section focuses specifically on iron electrodes. Electrochemical techniques suitable for energy storage research will be discussed in 1.7

As stated in 1.3 and 1.5, the iron electrode is of particular interest due to its suitability for utility scale energy storage and the attainability of long cycle lives [111]. Key drawbacks include poor energy density and propensity for wasteful hydrogen evolution on charging [87]. These drawbacks, specifically the hydrogen evolution reactions, are considered by this author as research challenges and will be discussed below. 
Hydrogen evolution (Equation 1.6-2) is the main reason for the poor faradaic efficiency of the iron electrode and occurs preferentially to the charging (iron reduction) reaction (Equation 1.6-1) as it is more favorable [19]:

$$
\begin{array}{lrr}
\mathrm{Fe}(\mathrm{OH})_{2}+2 e^{-} \rightleftharpoons \mathrm{Fe}+2 \mathrm{OH}^{-} & E^{o}=-0.870 \mathrm{~V} & \mathbf{1 . 6 - 1} \\
2 \mathrm{H}_{2} \mathrm{O}+2 e^{-} \rightleftharpoons \mathrm{H}_{2}+2 \mathrm{OH}^{-} & E^{o}=-0.828 \mathrm{~V} & \mathbf{1 . 6 - 2}
\end{array}
$$

Where $E^{0}$ is the electrode potential for the reduction reactions, measured in volts $(\mathrm{V})$.

In commercial cells this results in a round trip efficiency of approximately $55-70 \%$ and in a practical sense, the energy loss associated with hydrogen evolution means that cells require a $60-100 \%$ overcharge to reach true full capacity [22]. Additives can be used to make the hydrogen evolution reaction less favorable, by increasing the overpotential. This promotes the desirable charging reaction and results in improved round trip efficiency, which reduces the requirement to top up the electrolyte as it is lost through electrolysis [82], [86], [87].

The characteristics of iron electrodes and alkaline electrolytes will be discussed here.

\subsubsection{Iron as an Electrode Material}

The level of solubility of the iron reaction intermediate species in alkaline electrolyte (typically potassium hydroxide $\sim 5 \mathrm{M}$ ) has a significant effect on the iron electrode's characteristics, performance and cycle life. Yang et al describe the charge and discharge process as being a dissolution-precipitation reaction, involving a sparingly soluble ferrite ion intermediate, $\mathrm{HFeO}_{2}{ }^{-}$and an insoluble iron hydroxide, $\mathrm{Fe}(\mathrm{OH})_{2}$ [19]. These species were previously proposed by Öjefors [62], [101]. As the ferrite ion is sparingly soluble and readily transforms to iron (II) hydroxide on discharge, there is no opportunity for dendritic growth because active material cannot relocate to different physical locations in the cell from one charge to another. This also helps to ensure the reformation of the original high surface area structure on multiple charge/discharge cycles, aiding long cycle lives. The presence of this intermediary species also results in the poor high rate discharge performance of iron electrodes, as the precipitation of the iron hydroxide adjacent to the initial reaction sites has the effect of blocking the active surface and slows the rate of the 
reactions [26]. This relationship suggests a greater importance of the initial structure of the electrode and formation cycling will have less effect on the electrode structure when compared to other chemistries.

Various studies have demonstrated the suppression of the hydrogen evolution reaction by incorporating additives and reducing impurities within the iron electrode [54], [62], [75], [79]-[81], [112]. Sulfide additions in the form of iron sulfide have been reported to have a beneficial effect [19], [22], [67]. The overpotential for hydrogen evolution is also said to be increased along with capacity [113]. Manohar et al report that sulfide ions prevent passivation of the iron electrode and also increases long term cyclability by facilitating the conversion of undesirable magnetite into metallic iron during charge [83]. Additionally, it was found to be important if the sulfide compounds were incorporated into the electrode or the electrolyte. Lead sulfide and bismuth sulfide have also been used to produce a similar effect, as it appears to be the presence of the sulfide on the surface of the electrode which improves the iron electrode's performance, with unprecedented $96 \%$ charging efficiencies reported for $\mathrm{Bi}_{2} \mathrm{~S}_{3}$ additions [22], [113]. Bismuth oxide is also said to have a helpful effect on the overpotential for hydrogen evolution [81]. This is an active area of research with several recent publications and is of interest to explore in this author's work.

Simply switching from use of commercial grade iron to carbonyl iron, which does not contain common iron impurities such as manganese and phosphorous, produces a significant increase in the overpotential required for hydrogen evolution [22].

Kao et al note the large discrepancy between the theoretical capacity of the iron electrode (962 $\mathrm{mAhg}^{-1}$ ) and practically obtained values [114], said to be due to formation of a passive film [115], [116]. Their studies focus on improving the conductivity inside the iron electrode as a method of increasing the capacity of the overall electrode. Composite structures of iron/carbon and also of iron/copper have been produced with positive results over multiple cycles [114], [117]. 


\subsubsection{Electrolytes and Additives for Iron Electrodes}

$\mathrm{NiFe}$ cells typically use the following electrolyte chemistry: $25-30 \% \mathrm{w} / \mathrm{v}(\sim 5-6 \mathrm{M})$ potassium hydroxide solution with up to $50 \mathrm{gl}^{-1}$ of lithium hydroxide. Due to hydrogen evolution caused by electrolysis during charging, this solution will periodically require topping up with deionized water. There are also reports of beneficial effects of completely replacing the electrolyte in order to rejuvenate cell performance [26].

The addition of lithium (as $\mathrm{LiOH}$ ) to the electrolyte has several positive effects on cell performance; however the mechanism is not well understood, with Posada suggesting that further work is needed [87], [90]. Both electrode capacity and cycling efficiency are improved and lithium is also thought to be beneficial to the nickel electrode kinetics by 'mopping up' carbonates. For the Iron electrode, it is theorized that lithium ions result in a preferential pathway upon charging by formation of an intermediate compound with the iron oxide lattice such as $\mathrm{Li}_{x} \mathrm{Fe}_{y} \mathrm{O}_{z}$, which are then further reduced to form metallic iron and lithium hydroxide. However, Casellato et al note that potassium ions are still important for other reactions [118], [119].

Electrolyte composition can have a significant effect of performance of the iron electrode, in particular with respect to the parasitic hydrogen evolution reaction discussed in 1.6.1. Many different inhibitors have been evaluated over the years with the most complete study by Öjefors testing 28 different additives [62].

Interestingly, the majority of studies targeting hydrogen evolution focus on electrode additives as opposed to soluble electrolyte additives. Notable electrolyte-compatible additives include, iron sulfide, sodium sulfide and sulfur.

Sulfur compounds were first discussed in 1.6.2 and broadly the mechanisms for soluble additives are the same as electrode-incorporated additives. Various electrode and electrolyte compositions have also been investigated by Periasamy et al who also stated that incorporation of sulfur slows the $H_{2}$ evolution reaction by increasing the Fe-H bond energy [76]. The mechanism for sulfur containing inhibitors is discussed in detail by Jayalakshmi and Muralidharan in their writing in 'Corrosion' [120]. 
In other work, thiourea is used as a soluble electrolyte inhibitor at a low concentration of $0.05 \mathrm{M}$, however, Periasamy elected not to discuss its effects with respect to hydrogen evolution in detail [75]. Open Circuit Potential (OCP) studies were also performed and the author mentions negative results but does not elaborate why or investigate in terms of passivation [77]. This is unfortunate as the authors note that thiourea has been used successfully to alter hydrogen evolution kinetics on other materials and more research on its interactions with the iron electrode would be of value [121]-[123]. Vigdorovich et al show that thiourea increases the overvoltage of the HER on iron, albeit in aqueous solutions of ethylene glycol [121].

The Devanathan-Stachurski electrochemical double cell has been used by Amokrane et al to study hydrogen permeation into iron electrodes. This technique allows researchers to look in detail at the mechanisms involved in hydrogen evolution. Through this experimentation on iron electrodes in alkaline media, the authors determined that thiourea acted to inhibit the recombination reaction where two $H_{a d s}$ species combine (recombination reaction) [123]. The author considers this relatively recent publication in Electrochimica Acta to be high quality - and efforts to confirm previously conflicting results are convincing. there is merit in further investigation of the mechanism of thiourea to confirm these findings. Amokrane et al also used benzotriazole (BTA) for mechanistic comparison to thiourea. This is of interest to this author as benzotriazole is commonly used as an additive on brass and alloys of copper [124]-[127] and has been tested as a corrosion inhibitor on aluminium [128] and steel in acidic conditions [129]-[132]. Furthermore, benzotriazole has been used successfully within this research facility for corrosion performance on iron in unpublished work. Amokrane identified that the mechanism for benzotriazole was different than for thiourea, with benzotriazole acting to slow the Volmer reaction and thus inhibiting hydrogen permeation [123]. In copper alloys in pipes and heat exchangers, it is known to adsorb to form a stable single or multi layered film on the substrate surface and is subsequently well used [124], [133].

Other interesting experimentation using alkanethiol self-assembled monolayers has also been reported, unfortunately only to 25 cycles, making it difficult to assess the longterm performance of this additive. Good improvements in coulombic efficiency by 
reduction in the hydrogen evolution rate were shown and this author notes that this approach could possibly be combined with others to enhance the effects [79].

Recombination catalysts (often Pt based) can be used to recombine hydrogen and oxygen to make a sealed cell however this increases costs and does not improve round trip efficiency [66].

Whilst sulfur additives are reasonably well researched, most hydrogen evolution studies focus on electrode additives as opposed to soluble electrolyte additives. Use of novel electrochemical techniques could assist in developing understanding of less common soluble additives such as thiourea and benzotriazole. Accordingly, scanning electrochemical techniques, which offer the ability for spatial resolution, are discussed in 1.7 .

\subsubsection{Manufacture of the Iron Electrode}

\section{Negative iron electrode design}

Iron electrodes can be fabricated in several different manners. An ideal electrode is a porous collection of active material, where as much material is active and exposed to the electrolyte as possible whilst maintaining conduction to the current collector, to achieve the highest capacity. Power capability is also affected strongly by the electrode design and sintered electrodes are often favored for high power applications due to high surface areas. Historically, iron electrodes were produced on a nickel-plated steel scaffold although little academic detail about the legacy production route for the iron electrode exists [26].

\section{Pocket plate:}

This was the traditional method of producing the iron electrode involving pressing active material in to a fine, nickel-plated mesh. Pure iron was dissolved in concentrated sulfuric acid resulting in $\mathrm{FeSO}_{4}$, which was dried in air and heated to produce $\mathrm{Fe}_{2} \mathrm{O}_{3}$. Remaining sulfate was washed off and the active material was dried in a reducing atmosphere to produce a mixture of $\mathrm{Fe}_{3} \mathrm{O}_{4}$ and $\mathrm{Fe}$. The material, mixed with various additives, was then 
then mechanically inserted into the formed nickel plated steel strips and crimped in to place [26]. This process is mechanically intensive and would not be suitable for modern mass production. Mabbett et al comments that it was not until development of $\mathrm{NiCd}$ batteries that nickel electrode production was advanced [37].

\section{Pressed plate:}

Pressed electrodes imply that some amount of plastic deformation is being used to form a stable electrode. For a batch process, a press would be used and a roll could be suitable for continuous or semi-continuous applications. As high temperature sintering is not used, pressed electrodes are not cost intensive to manufacture [22]. Pore formers can be used to increase surface area and binders used to improve adhesion, these are mixed in prior to pressing [80]. Pressed electrodes are typically heated to $<200{ }^{\circ} \mathrm{C}$ during pressing [80], [81], [83], [134].

Manohar et al reported that use of high purity carbonyl iron - derived from the decomposition of iron pentacarbonyl - results in improvements to charging efficiency through a reduction in hydrogen evolution. This is attributed to reduced content of common impurities (manganese, sulfur and phosphorus) which act to decrease the overpotential for hydrogen evolution [22], [80]. There do not appear to be any negative implications of using the higher purity carbonyl iron and it is readily available in spherical powders, so will be used throughout this author's research.

There is a reasonable amount of literature published in recent years on the topic of low temperature electrodes containing binders, although some authors use lengthy processing times during manufacture of electrodes [90]. In this same work, Posada comments on the level of uncertainty in results obtained, which exposes the difficulty in producing repeatable electrodes on a lab scale such that the effect of additives can be successfully determined. This author also notes that variability is not routinely discussed in results presented in electrode manufacture literature. 


\section{Sintered plate:}

A slurry or dry iron powder can be heated at temperatures approaching the melting temperature in order to sinter the iron. Yang et al, referencing much earlier work by Vassie and Tseung, noted that sintered electrodes were likely to be more mechanically robust than pressed electrodes and this has importance for attaining long cycle lives [19], [135]. In this work, Vassie and Tseung refer to electrodes being produced from either iron or iron oxide, but both routes require a reducing atmosphere $\left(H_{2}\right)$ to avoid excessive oxidation of the iron active material at sintering temperatures.

The temperatures involved in sintering iron are inherently expensive and the author considers that, ideally, a temperature should be chosen such that iron could be sintered evenly in less than five minutes, to be sympathetic to the needs of mass production via a semi-continuous roll to roll production line. Sintering in reviewed literature was not achieved in less than 15 minutes [19], [75], [76], [135]. This author considers there to be merit in research into rapid sintering of iron electrodes. Furthermore, whilst sintering is generally considered to be cost-prohibitive, performance gains reported by Mabbett et al may outweigh these drawbacks, especially when compared to current pocket plate electrodes. This author shares the view of Mabbett et al that performance gains in other chemistries have not been applied to iron electrodes and that sintering of iron for energy storage warrants further investigation [37]. Yang et al have also evidenced the potential for pore formers to be used when sintering iron and, as discussed previously, good results have been attained through use of high purity carbonyl derived iron powder [19].

Additionally, an activating agent or flux may depress the sintering temperature, although effects on electrode performance would also have to be considered [136], [137].

\section{Nanostructured electrodes}

The highest performing electrodes in literature utilized nano-structuring to achieve gravimetric capacities near the theoretical maximum attainable for iron [20], [29], [102][104]. Whilst the results reported are impressive, none of the literature reviewed contained descriptions of processes which could be scaled to high volume production either on grounds of complexity or cost. This author values scalability over outright 
performance so will not discuss this literature further. Layered electrodes, as discussed in 1.5.2, are also an interesting concept which could be applied to iron electrodes.

\section{Rapid heating and mass production}

For mass production, iron electrodes must be suited to economic manufacture via a semicontinuous roll to roll process. A key enabler for this is rapid heating, either to achieve pressed plate or sintered electrodes [138], [139]. This methodology has been applied by colleagues to the manufacture of printed photovoltaics using near infrared (NIR) heating [140]-[142]. Generally, rapid heating methods (NIR, Microwave, etc.) offer significant increases to the power input to a target material or substrate but may result in less even heating across the surface when compared with conventional technologies such as convection ovens.

This is a promising area of research which has seen very little attention within energy storage but is considered to be essential for industrialization. These challenges will be considered when this researcher attempts to manufacture electrodes.

Additionally, printing alone is an important enabler for mass production. Printing allows for large areas of substrate to be coated with an active material, in a similar way to how steel is coated on a continuous coating line - clearly an operation which would not be feasible if it were done manually. Various methods for printing exist, from screen printing to stereolithography. These are not discussed in detail, however this author argues that any research which has mass production as a focus should use inks which are capable of being printed, even if printing is not employed during the research. This enables the future capability for others to reproduce similar work using printing.

\subsection{Scanning Electrochemistry for Energy Storage Research}

\subsubsection{Introduction}

For the energy storage focused reader, these technologies require a brief introduction. Typically employed in corrosion studies, scanning electrochemistry is capable of mapping localized events which are only area-averaged in traditional analytical 
techniques such as cyclic voltammetry. A probe is manipulated across a surface in either two or three dimensions whilst recording data, allowing for construction of a map of surface activity by location with respect to time.

The Scanning Vibrating Electrode Technique (SVET) and Scanning Kelvin Probe (SKP) are both capable of operating in this manner. The SVET uses a platinum microdisc electrode which is vibrated normal to the surface of a sample immersed in a solution, allowing a current density map to be produced during scanning. The SKP uses a gold probe which is scanned over a sample in atmospheric conditions to measure Volta potential difference. This can produce a map of surface activity. Neither of these techniques have been evidenced to perform on alkaline electrode for energy storage, yet, theoretically, both could be used to map heterogeneity across electrodes. Additionally, by scanning over time, charge and discharge activity including 'hotspots' and degradation could be studied.

Whilst an in-depth view of the scanning electrochemistry as an analytical technique is given in Chapter 2, the discussion here is of relevant literature underpinning these techniques and is included to demonstrate the crossover between the typical corrosion studies and the proposed energy storage studies.

\subsubsection{Scanning Vibrating Electrode Technique (SVET)}

The SVET is typically used to study corroding metal surfaces which are fully immersed in saline solutions. This technique has been discussed in a range of literature and presented at many international conferences [143]-[148]. It is considered to be an iteration on the Scanning Reference Electrode Technique (SRET), with early work on defining the technique discussed by McMurray and Worsley in 'Advances in Chemical Kinetics' [149]. Study of cut-edge corrosion in zinc-based coatings is common [150][153]. Use of a probe allows for determination of individual features (Figure 8), relating these to a co-ordinate position and monitoring them as they progress through time. Use of a probe vibrating in an axis normal to the sample, yet close to the surface, allows for measurement of the current flux in solution across the surface and thus determination of current density. 


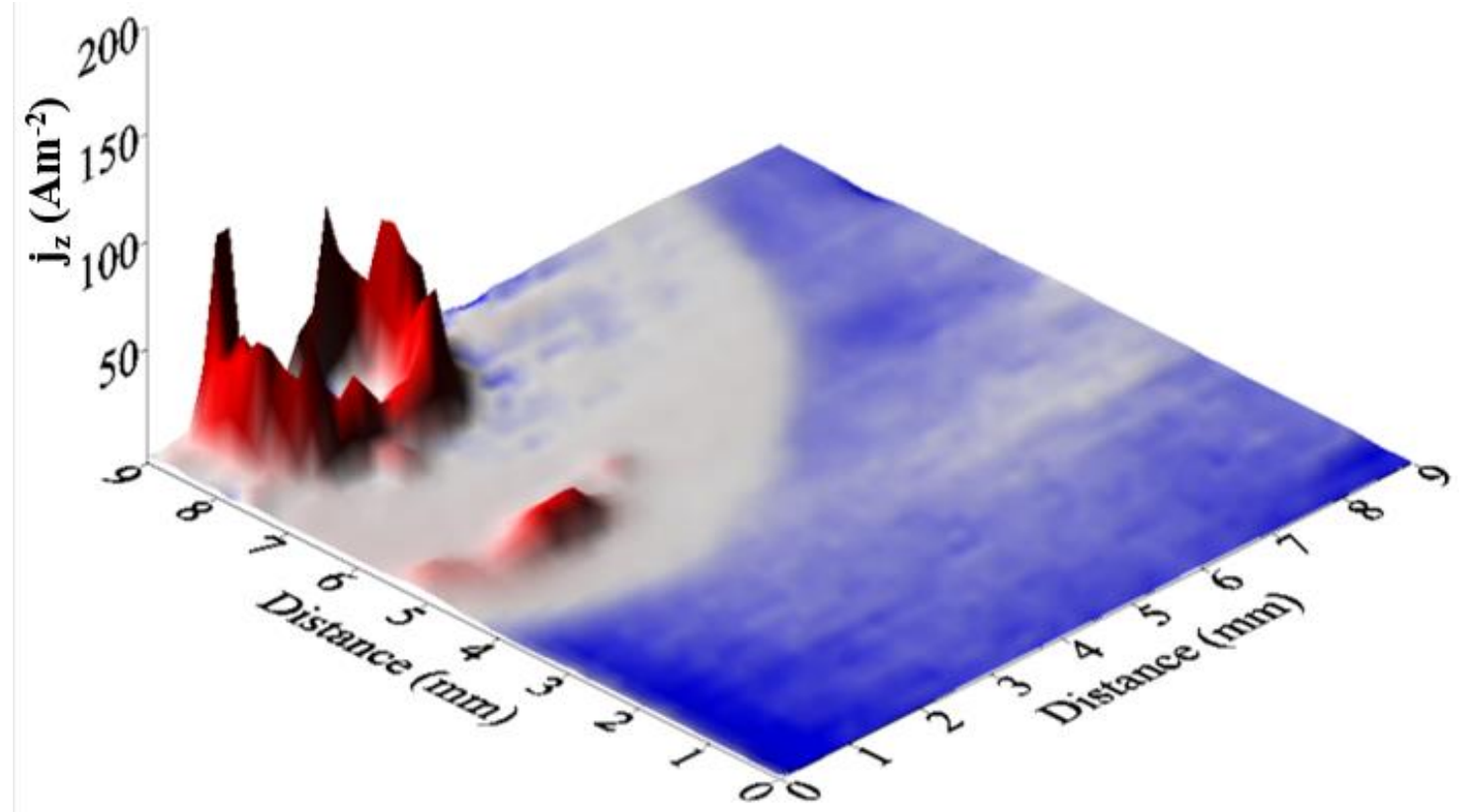

Figure 8 SVET-derived image of corrosion activitiy [154]

This technique has not been used previously in a study focused on energy storage. It has, however, been used for studies on hydrogen evolution on polarized corroding magnesium substrates [155]-[159]. This is a similar application to that envisaged by this author. Adaptation will be required for SVET to operate in the strong alkaline conditions typically associated with iron electrodes.

\subsubsection{Scanning Kelvin Probe (SKP)}

The SKP is commonly used to investigate delamination of coatings through the actions of corrosion. Unusually, the technique can be used to 'see' through organic films making it powerful for investigating atmospheric corrosion [160]-[163]. Filiform corrosion is also investigated using SKP [164], [165]. First developed by Stratmann et al, the technique is now widely accepted and used in a variety of academic institutions despite the requirement for highly specialized equipment [166]-[169].

As the SKP operates in atmospheric conditions and does not require an electrolyte bath for operation, it can also be used to look at heterogeneity in oxide layers. Examples of this include studies as a result of corrosion by fingerprints (Figure 9) [170]-[172] and effects of hydrogen evolution/diffusion [173]-[178]. Williams et al described the effects 
of atomic hydrogen on surface oxides in detail in the 2013 publication in Electrochemistry Communications. They show that the interaction of diffusing atomic hydrogen with the surface oxide layer in an iron electrode produces changes to the local Volta potential which is detectable by the SKP. Where the partial pressure of oxygen is kept constant, a ratio between $F e^{I I} / F e^{I I I}$ is established where atomic hydrogen diffusing through the substrate reduces $F e^{I I I}$ at the metal-oxide interface, which is then re-oxidized by atmospheric oxygen (Figure 10). An equilibrium is quickly reached such that there is a detectable deviation in the local Volta potential proportional to the surface oxide ratio in areas where atomic hydrogen is present in the bulk substrate. This Volta potential depression effect can be calculated using the Nernst equation [173]. Palladium can also be used to quantify this effect [176].

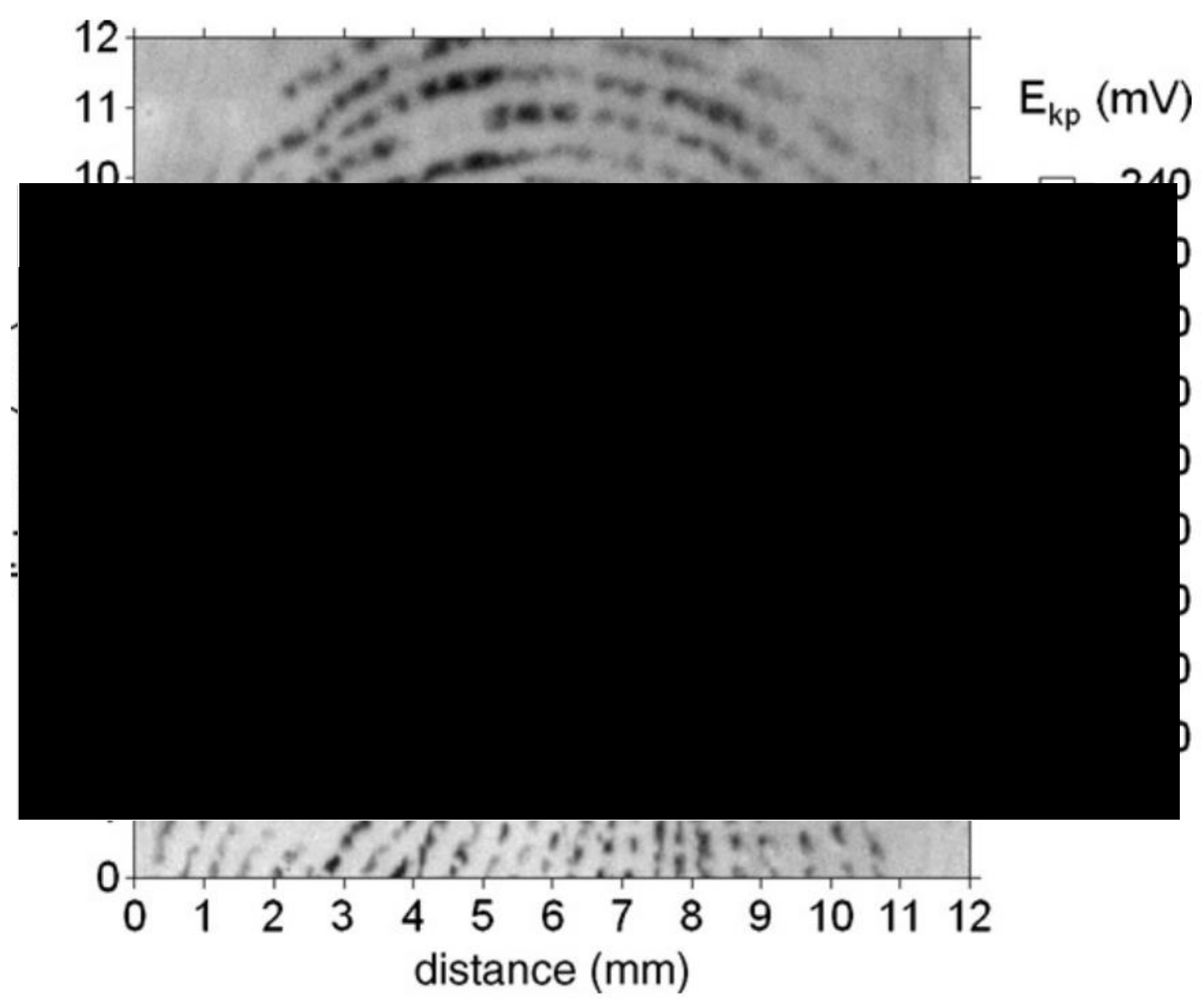

Figure 9 Scanning Kelvin Probe scan of a latent fingerprint [172] 


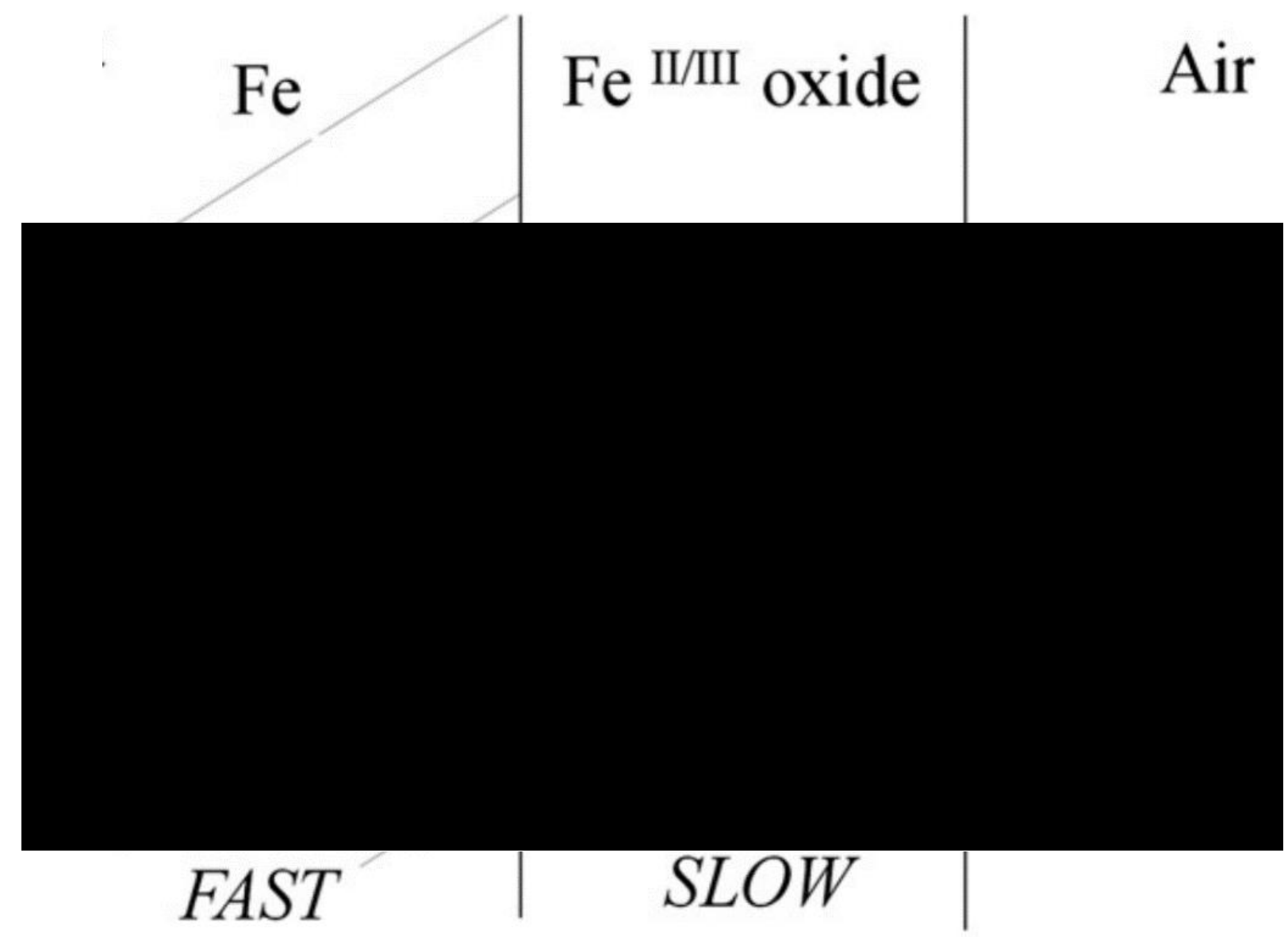

Figure 10 Electron transfer reactions on the iron surface during hydrogen evolution [173]

Operation of the SKP is similar in some ways to the SVET (1.7.2), in that a probe is vibrated normal to the sample surface. However, the gold probe itself is the reference and as such, an external reference is not required. External circuitry is used to produce nullcurrent conditions by continuous adjustment of a dc bias voltage. This bias voltage is commonly referred to as the kelvin probe potential $E_{k p}[170]$. This operation is repeated as measurements are taken across the surface of a sample in order to produce Volta potential maps.

Given that hydrogen evolution is of interest in this research, it may be possible to develop understanding of the HER and the mechanisms of inhibition through use of the SKP. 


\subsection{Research Aims}

Iron electrodes represent a large topic with significant scope for potential research due to a relative lack of previous investigations when compared to other energy storage materials. As a result of a review of literature, the following areas requiring research attention and subsequent research aims were identified:

1. There are several recent studies focused on the manufacture of electrodes (1.6.4), however the objectives are typically limited to improving electrode performance through efficiency or utilization gains. Whilst promising results have been achieved by a low temperature manufacturing process, where binders are used to form stable electrodes, speed of manufacture is still relatively slow. Research with a focus on manufacturing speed is required to enable economic roll to roll production. Accordingly, this researcher sees merit in investigating a process to support the rapid manufacture of iron electrodes using an inherently cost efficient low temperature route with polymer binders.

2. Various studies have used sintering to produce a structurally stable iron electrode. As noted in the 'sintered plate' section of 1.6.4, sintering times are in excess of 15 minutes. For the purpose of manufacturability, a reduction in the time taken to sinter is essential to enable mass production. To address this, high temperature rapid sintering is investigated, with the intention to reduce the sintering time below 15 minutes. Due to their physical bonding, these electrodes are anticipated to offer the longest cycle lives.

3. Scanning electrochemistry has found many applications in brine electrolytes for corrosion studies and other closely related fields, however, studies in strong alkaline conditions have not been possible due to difficulties in mounting samples and containing the aggressive electrolytes. In order to access the novel analytical capabilities of scanning electrochemistry, this method should be developed further. This will involve the design and testing of equipment required to enable the use of scanning electrochemical techniques in the strongly alkaline conditions typically associated with iron electrodes. 
4. Detail can be gained on detrimental secondary iron electrode 'hydrogen evolution' reactions through use of the of the Scanning Vibrating Electrode Technique. Use of scanning electrochemistry in these strong alkaline conditions will enable this researcher to assess the homogeneity of electrode reactions and compare the performance of different inhibitors, however to complete this study, verification will be required comparing the results obtained with a traditional technique to those obtained using the Scanning Vibrating Electrode Technique. This technique will also be used to study previously manufactured electrodes.

5. Further investigation in to the mechanism of the parasitic hydrogen evolution reaction discussed in 1.6.1 will be completed using the Scanning Kelvin Probe. In particular, the study using the Devanathan-Stachurski electrochemical double cell to assess the mechanism behind the action of thiourea (discussed in 1.6.3) is of interest and will be investigated using the Volta potential depression effects of atomic hydrogen, which the Scanning Kelvin Probe is capable of detecting. This will be used to investigate and verify previously proposed mechanisms for hydrogen evolution inhibitors, with the advantage of spatial resolution. 


\section{Chapter 2: Experimental Techniques}

\subsection{Preparation and Testing of Iron Electrodes}

Various methods exist for producing iron electrodes, including screen printing and gravure printing, or more simply, tape casting. In this author's research, electrodes were tape cast on to steel substrates. The substrates used had undergone a variety of surface preparations, as detailed below.

\subsubsection{Steel Substrate Surface Preparation}

Various samples throughout this work were mechanically roughened to alter their characteristics, prior to being utilized. The substrate was a low carbon steel, $0.3 \mathrm{~mm}$ thick, supplied by Tata Steel Europe, the requested grade was 'DX54'. The exact chemistry was determined by the supplier and is listed in Table 1. Due to the surface treatment, these samples will henceforth be known as, 'as received', 'ground' and 'wire brushed'.

The substrate preparation methods were devised to replicate processes which could be used on an industrial manufacturing line. 
Table 1 Tata Steel DX54 low carbon steel chemical analysis, as reported via a personal comunciation to this authour

\begin{tabular}{|l|l|l|l|l|l|}
\hline Plant: & Port Talbot & Cast No: & $16541 \mathrm{PT}$ & Roll No: & 23200010 \\
\hline Element: & Wt.\% & Element: & Wt.\% & Element: & Wt.\% \\
\hline Carbon & 0.0740 & Boron & 0.0002 & Vanadium & 0.0010 \\
\hline Silicone & 0.0020 & Copper & 0.0130 & Tungsten & 0.0000 \\
\hline Manganese & 0.4650 & Nitrogen & 0.0126 & Zirconium & 0.0000 \\
\hline Phosphorous & 0.0100 & Niobium & 0.0010 & Sodium & 0.0220 \\
\hline Sulfur & 0.0090 & Lead & 0.0000 & Nickel & 0.0150 \\
\hline Chromium & 0.0120 & Tin & 0.0020 & Aluminum & 0.0230 \\
\hline Molybdenum & 0.0010 & Titanium & 0.0010 & Arsenic & 0.0010 \\
\hline Cobalt & 0.0040 & & & & \\
\hline
\end{tabular}

\section{As received substrate}

Samples described as 'as received' have not undergone surface modification. Surface contaminants were removed using organic solvents prior to testing.

\section{Ground substrate}

Ground samples were mechanically abraded using an abrasive grinding wheel. This was rotated above the sample using a Jones-Shipman 540 surface grinder (Figure 11) and bed fed over the sample in order to abrade the surface. The manufacturer of the grinding wheel was Flexovit (Figure 12), however, the specification was not known. The wheel was lowered in increments of $0.00254 \mathrm{~mm}(0.0001$ inches $)$ from first contact with the workpiece, however it was not possible to accurately record the depth of cut.

All samples were prepared in this manner on one occasion to ensure there was minimal variation between the samples. 


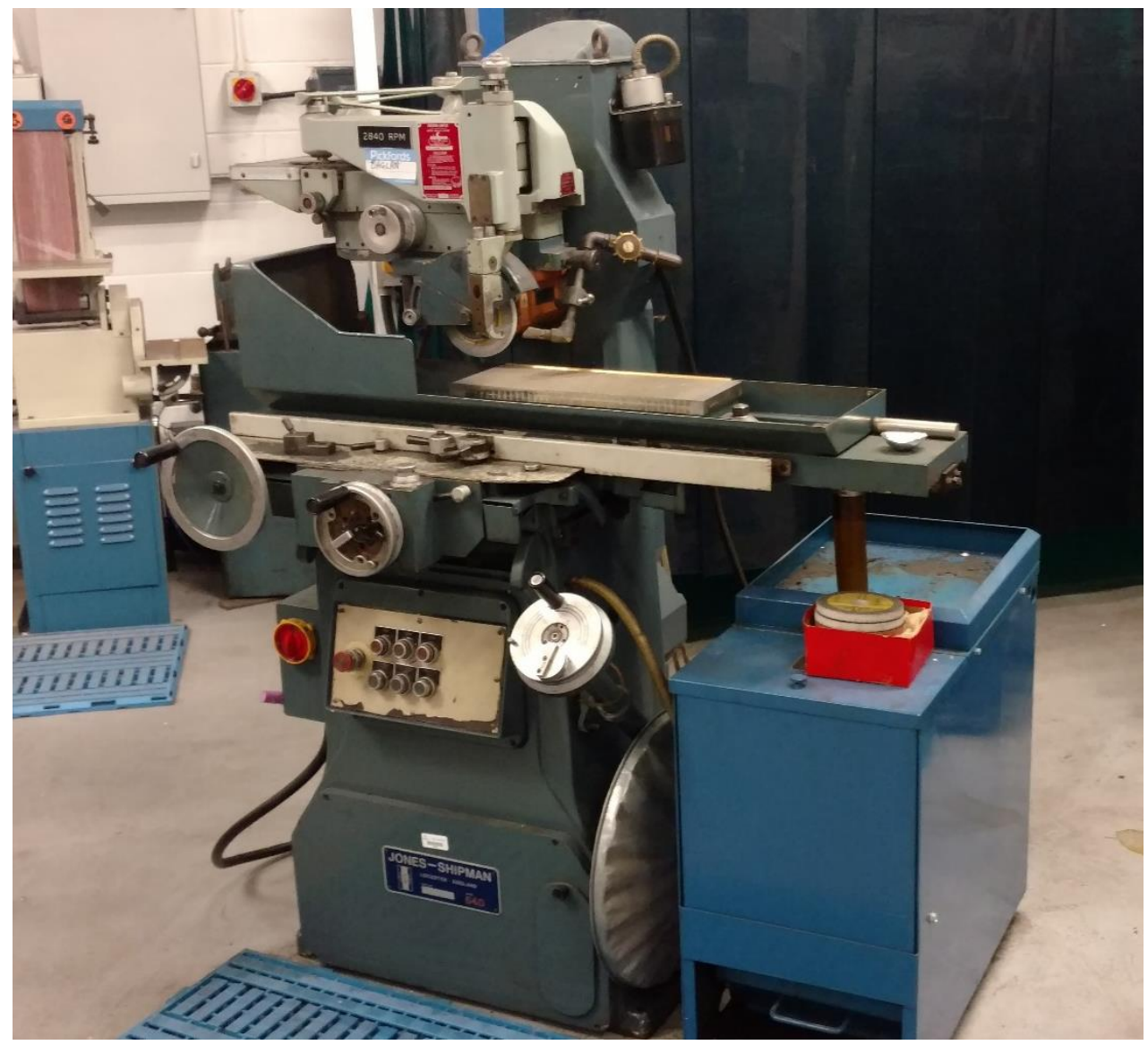

Figure 11 Jones Shipman 540 surface grinder fitted with magnetic bed chuck

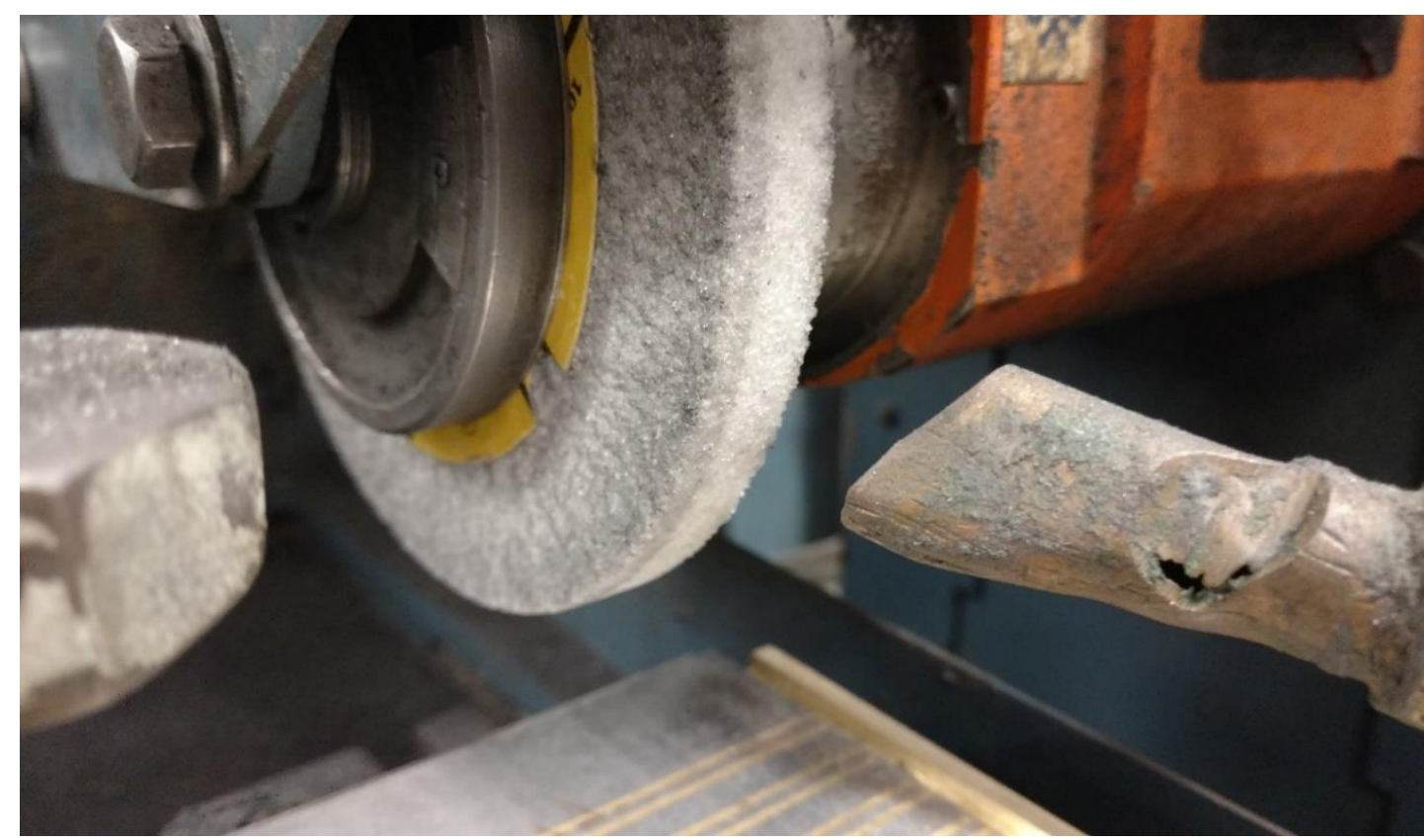

Figure 12 Flexovit grinding wheel above magnetic bed chuck 


\section{Wire brushed}

Wire brushed samples were mechanically abraded using a crimped cup wire brush. A Bridgeport Series I 2HP (manufactured by Adcock and Shipley) vertical milling machine (Figure 13) was used to spin a coarse-textured, thick gauge wire brush at $3000 \mathrm{rpm}$ above the sample. The brush was manufactured by Osborn and had a diameter of $90 \mathrm{~mm}$, however the exact model number could not be identified (Figure 14). The brush was held at $30^{\circ}$ from vertical to reduce the surface area acting on the workpiece and thus reduce heat input and potential for burning the substrate. It was not possible to record the downward pressure or depth of cut of the machining operation.

All samples were prepared in this manner on one occasion to ensure there was minimal variation between the samples. 


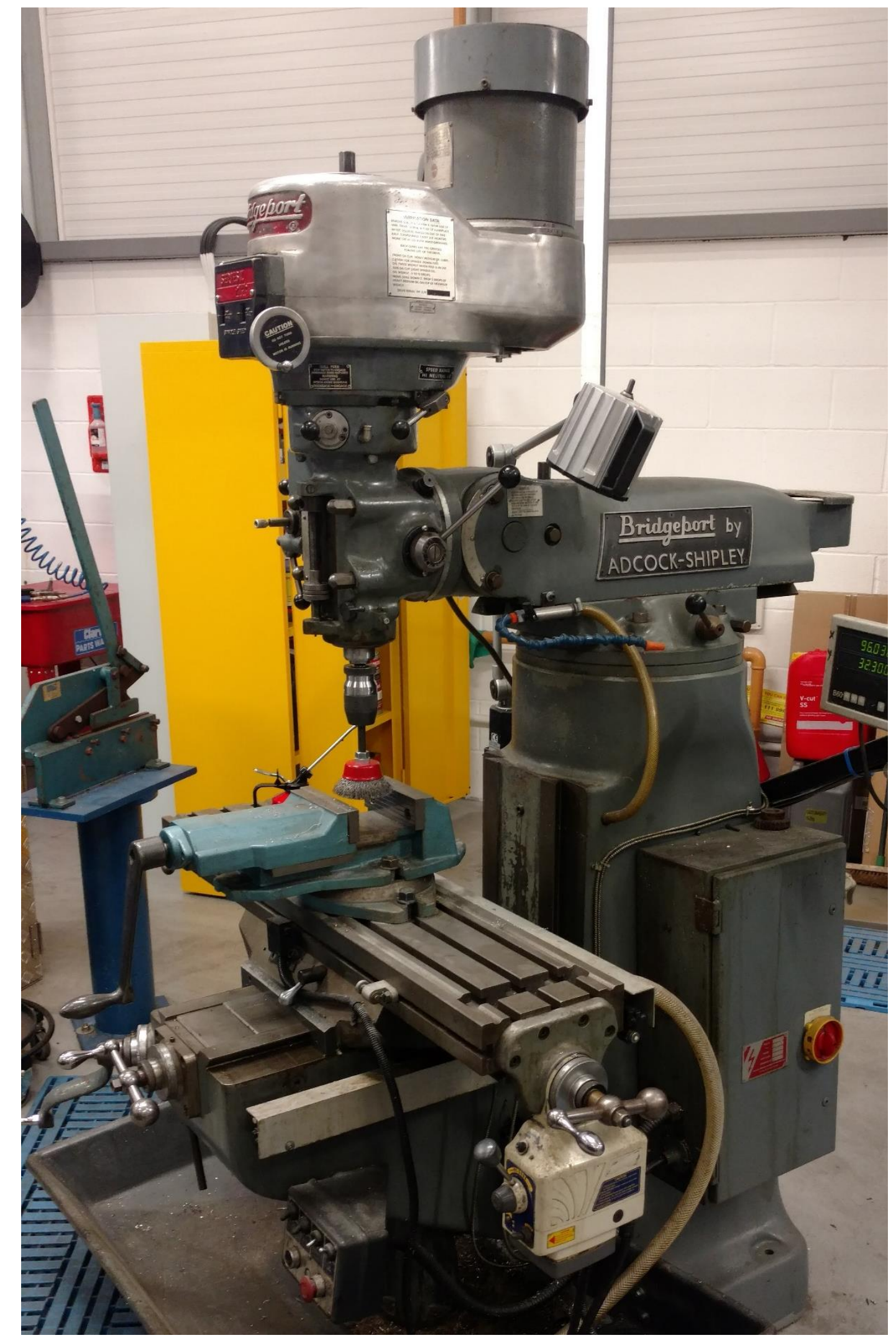

Figure 13 Adcock and Shipley manufactured series I milling machine 38 


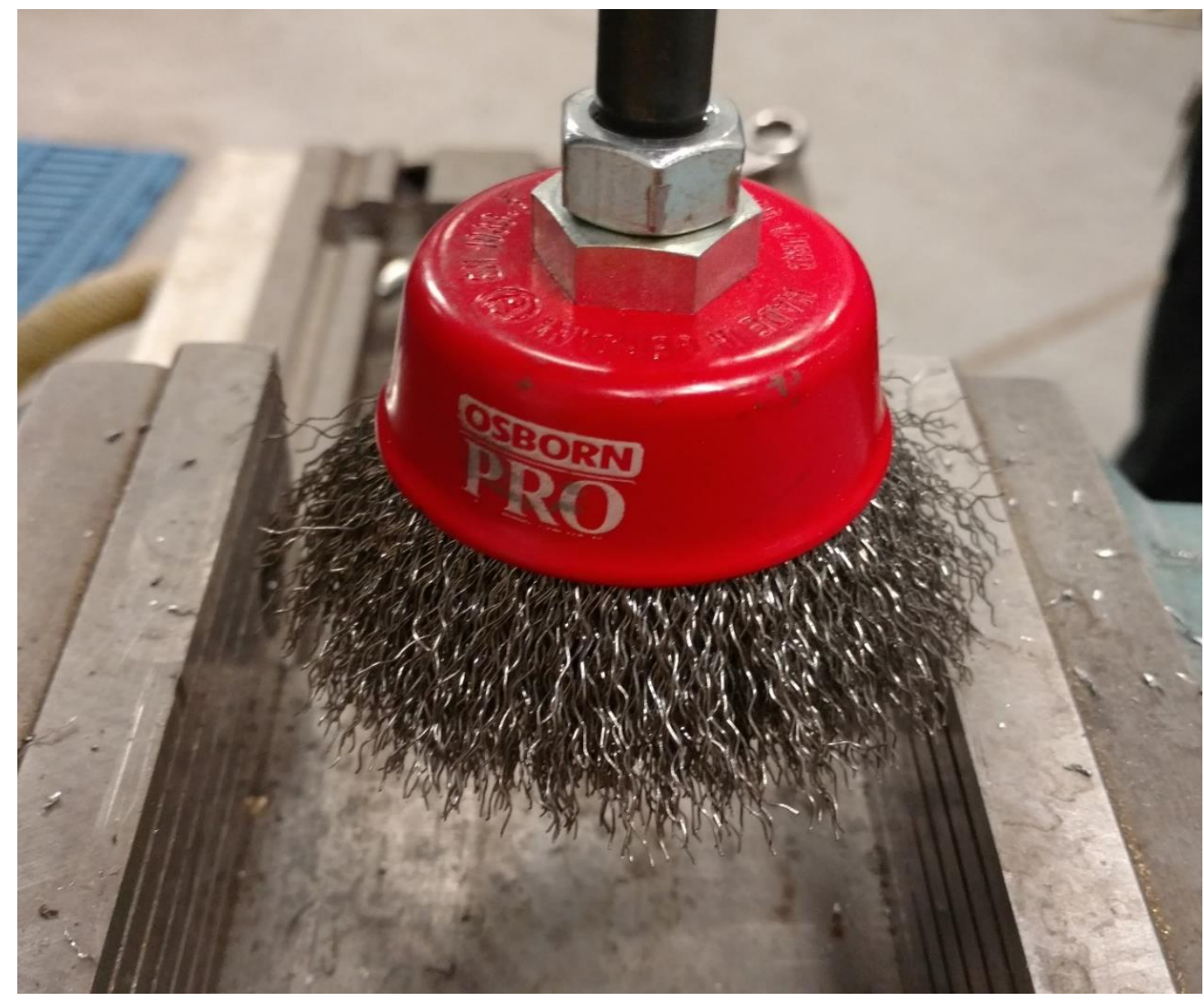

Figure 14 Osborn Pro crimped wire cup brush

\subsubsection{Iron Inks}

Manufacturer supplied information on the iron inks used in this study are documented in Appendix A. discussion around this author's specification for the iron inks is included in the relevant experimental chapters.

\subsubsection{Tape Casting (Coating) of Iron Inks}

Iron electrodes examined in this thesis were produced by tape casting. This is a simple coating process which can be completed without any dedicated or specialist equipment. Tape of a known thickness was used in order to offset a glass rod used for coating, to produce a wet film of a consistent depth, as shown in Figure 15. Drawing the iron ink across the substrate using the glass rod removes any excess ink and leaves the sample area selected coated with a consistent amount of ink. Tape can be layered as required to 
create a thinner or thicker coating. The location of the tape defines the surface area which will be coated. This ensures reasonable consistency between samples.

In this work, substrates were surface-modified as discussed in 2.1.1 and then cleaned with acetone. Acetone selected as an aggressive solvent to ensure cleanliness from the surface treatment process. As mentioned above, adhesive tape was used either side of the area to be coated and a glass rod was used to spread the ink. This author deposited the ink in advance of the glass rod and the action of drawing the rod over the sample/tape, perpendicular to the tape guides, produced a coating of constant thickness and removed any excess ink.

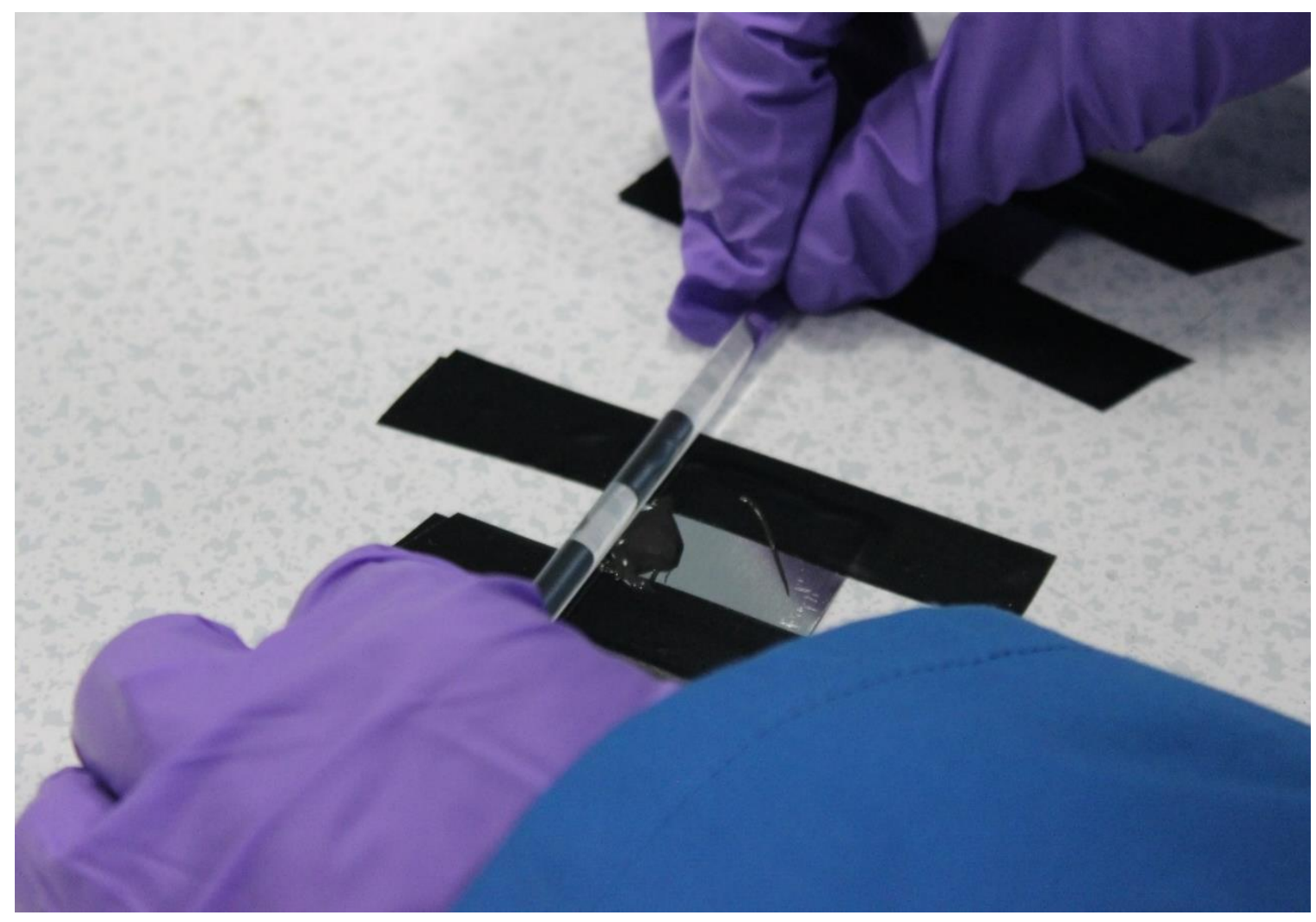

Figure 15 Example of tape casting using a glass rod

To ensure no contamination was introduced during tape casting, the glass rod and top surface of the tape were cleaned prior to coating, also with acetone. The adhesive tape (3M Scotch electrical tape $0.13 \mathrm{~mm}$ thick supplied by RS Components) was pressed down firmly to ensure air was not entrapped below it and increase repeatability of the tape casting process. 


\subsubsection{Heating Methods}

Various heating methods were utilized in this work as described below. This was required in order to cure or sinter the ink to form solid electrodes capable of being electrochemically tested. Below, 'low temperature' refers to heating methods which would be unable to reach the temperatures required to sinter iron, where binders would be used to ensure a coherent electrode. Alternatively, 'high temperature' heating methods were used with the aim of producing mechanically stable electrodes, without the use of binders, via sintering.

\section{Hot plates - low temperature}

For simplicity, heating below $300{ }^{\circ} \mathrm{C}$ was performed with commonly available thermocouple controlled hot plates. These use an electric heating element to heat a plate on which the sample can be placed directly. They were capable of curing the inks prior to further heating with other methods. Unless described otherwise, these were allowed to reach their set temperature before samples were placed on them. This equipment is readily available in laboratories and was chosen on the basis of accessibility.

\section{Near infrared - low temperature}

As discussed in 1.6.4, near infrared heating (NIR) is a powerful heating technique. The equipment available to this researcher was an AdPhos NIR/IR Coil lab LV2. This NIR oven (Figure 16) heats using tungsten halogen emitters that output at 250-2500 nm with the peak emissions occurring at 800-1200 nm. During operation a sample would be placed on the bed and the equipment would be programmed accordingly to manipulate the sample from outside the machine, past the NIR lamps and out the other side of the machine. During use, both the line speed of the conveyer (in $\mathrm{m} \mathrm{min}^{-1}$ ) and the percentage intensity of the lamps (0-100 where maximum lamp power is $25 \mathrm{~kW})$ could be programmed. The physical layout of the machine is such that a $2 \mathrm{~m} \mathrm{~min}^{-1}$ line speed equates to a $12.5 \mathrm{~s}$ residence time directly under the NIR emitters. There was no facility to accurately measure the surface temperature of the sample. 


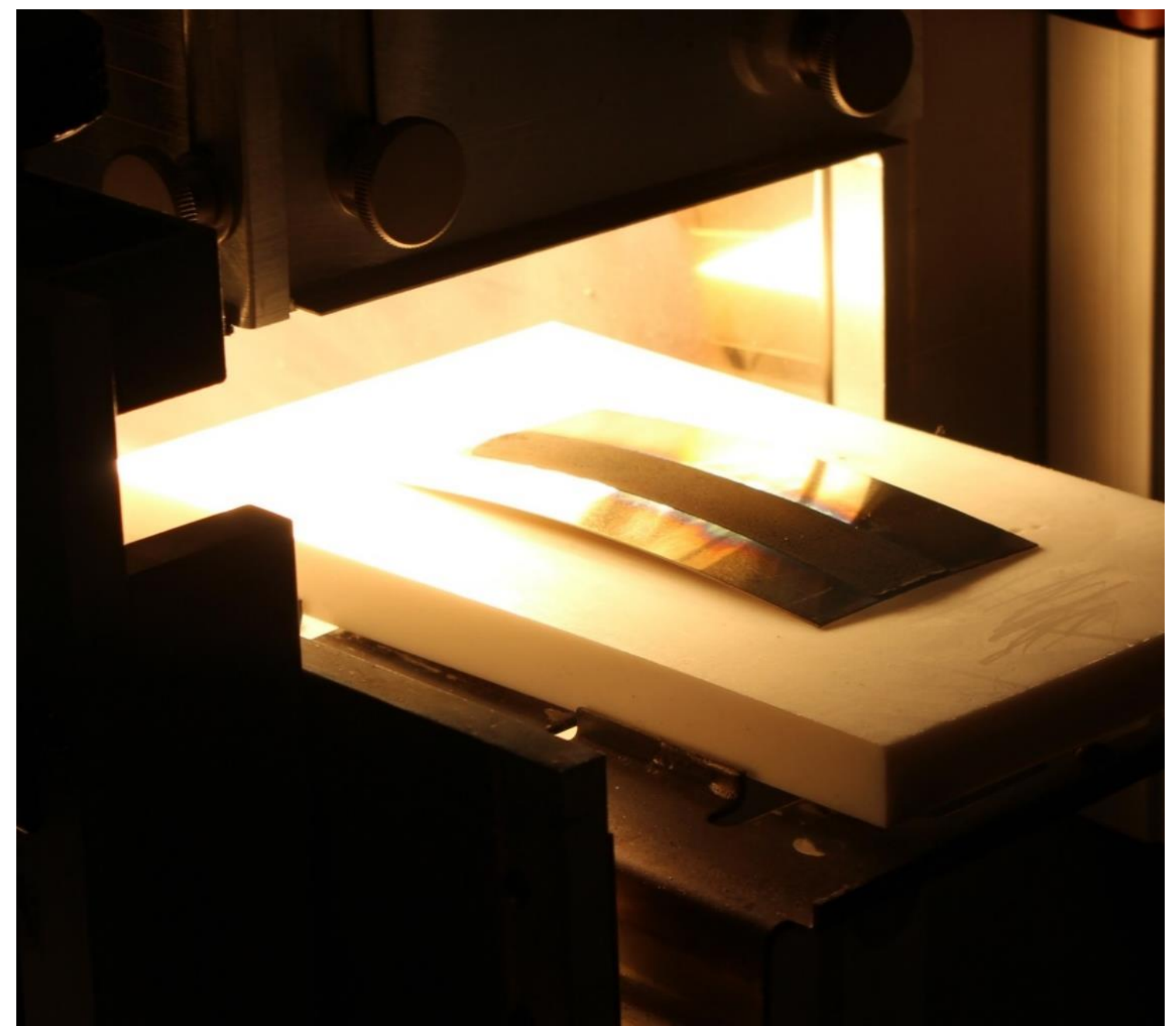

Figure 16 NIR heating of iron-containing ink on a low carbon steel substrate

Colleagues manufacturing printed photovoltaics have reported successes using the equipment. As a result, it was selected by this researcher to see if it could have other applications. The rapid heating aspect was advantageous as a large number of samples could be processed in the available time.

\section{Furnace with nitrogen atmosphere - high temperature}

To reach the temperatures required to sinter iron electrodes, the aforementioned AdPhos NIR was not sufficient. A preliminary effort was made to sinter a sample using a conventional resistance heating chamber furnace. A computer-controlled table top Nabertherm N 7/H furnace was available and was fitted with a gas box through which Nitrogen gas could be flowed. Unfortunately, the preliminary attempt to sinter an 
electrode $\left(1000{ }^{\circ} \mathrm{C}\right.$ for 30 minutes) was unsuccessful as the sample was extremely heavily oxidized during this operation. It was not possible to use a reducing atmosphere or a gas box with better sealing in this apparatus. Work using this equipment was not continued.

\section{Infrared furnace with reducing atmosphere - high temperature}

A water-cooled infrared furnace was available to the researcher. This furnace was part of a 'hot dip simulator' (HDS), typically used for investigating zinc galvanizing conditions [186]-[190]. It is generally used for preheating steel prior to dipping in into a molten zinc bath to emulate a steel coating line. The furnace is fitted with infrared lamps inside a water-cooled jacket. When illuminated, the infrared lamps emit significant amounts of heat. Sealed 'gates' above and below the furnace allow the use of a controlled atmosphere. A 5\% hydrogen in nitrogen atmosphere, commonly referred to as ' $\mathrm{HNX}$ ' (BOC gasses), was used for reducing conditions during all investigation with the furnace. Additionally, jets of pure nitrogen could also be used to cool the sample rapidly. The equipment used by the researcher (Figure 17) was originally manufactured by Rhesca in Japan and imported by Iwatani. Due to a fault, the original control system had been replaced with a custom aftermarket computer-controlled system, which was capable of controlling the heating rate, sample loading, atmosphere and dwell times, in a similar manner to the original control system.

To operate the furnace, a K-type thermocouple was spot welded on to each sample (the location was kept consistent to aid repeatability) and this was used to inform the computer of the current temperature of the sample during heating. Prior to the sintering operation, the sample was attached vertically to a drive rod which was situated within the loading chamber (Figure 18 top, directly above glowing furnace).

The rapid heating capability of this equipment was of interest to this researcher due to the aim to sinter samples in less time than has been reported in literature (see aims in 1.8). 


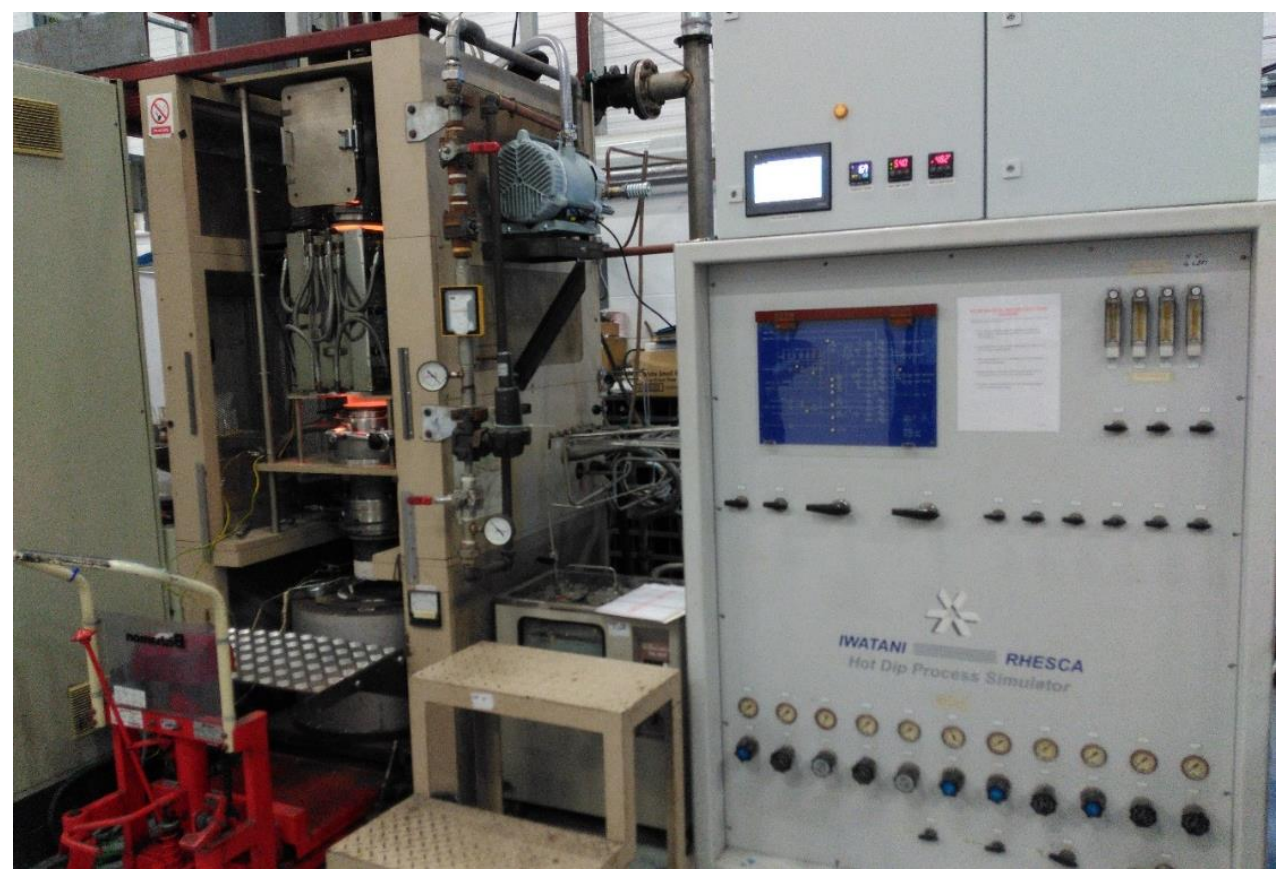

Figure 17 Iwitani Rhesca Hot Dip Process Simulator. Furnace - left. Control system - right

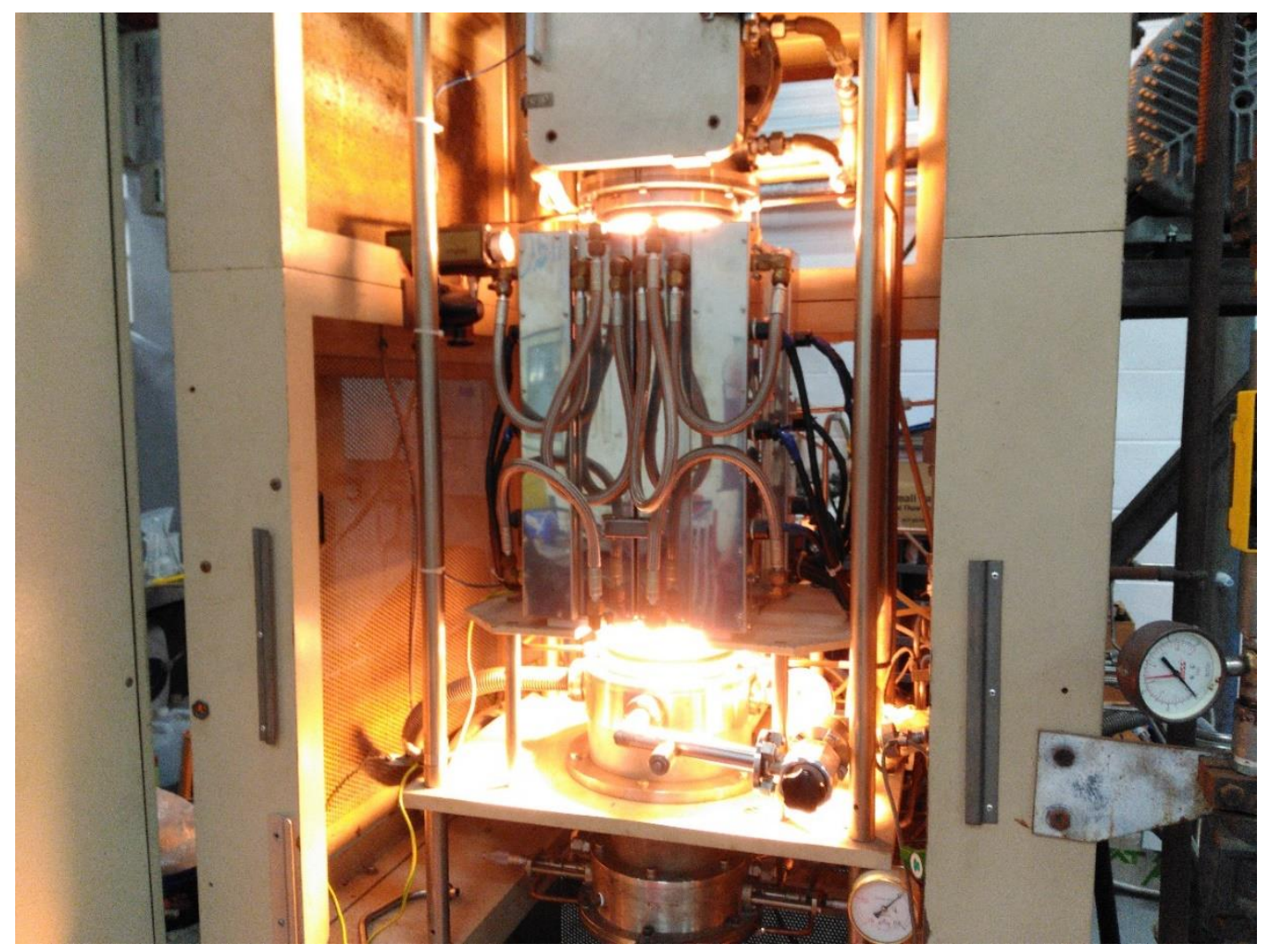

Figure 18 HDS Infrared Furnace during operation 


\subsubsection{Electrochemical Cycling of Electrodes}

In order to test the electrodes manufactured, charging and discharging experiments were performed using Electrochemical cycling of half cells. A 64 channel Arbin SCTS battery cycler was used and this equipment was operated under galvanostatic conditions at room temperature, as is typical for the iron electrodes which were manufactured and studied. Suitable voltage limits and $\mathrm{C}$ rates were obtained from literature [26]: cells were cycled from $0.8-1.2 \mathrm{~V}$ (vs. $\mathrm{Hg} / \mathrm{HgO}$ ) and a $0.2 \mathrm{C}$ rate was used; however, charging was discontinued if the voltage limit had not been met within 7 hours. The current and voltage was recorded at $<1$ second intervals through the duration of cycling.

Iron electrodes selected for cycling were cycled in a three-electrode setup, acting as the working (negative) electrode. The counter (positive) electrode was obtained from a commercial $\mathrm{NiOOH}$ electrode, from a Changhong Nickel Iron battery and a $\mathrm{Hg} / \mathrm{HgO}$ electrode $\left(\mathrm{E}_{\mathrm{Hg} / \mathrm{HgO}}^{\mathrm{o}}=+0.098 \mathrm{~V}\right.$ vs. $\left.\mathrm{NHE}\right)$ was used as a reference electrode. It was ensured that the counter electrode was sufficiently oversized such that it did not limit the operation or performance of the working electrodes.

Cells were assembled immediately prior to manufacture with an electrolyte composing of $5 \mathrm{M} \mathrm{KOH}$ with an addition of $0.05 \mathrm{M} \mathrm{K}_{2} \mathrm{~S}$. This author recognizes contribution from Dr. Jorge Omar Gil Posada (University of Sheffield) for assistance in the electrochemical cycling of electrodes. The $\mathrm{K}_{2} \mathrm{~S}$ was included on their recommendation.

\subsection{Scanning Vibrating Electrode Technique (SVET) for Investigation of}

\section{Battery Electrodes}

Past studies have utilized the SVET for corrosion investigations on metal surfaces fully immersed in saline solutions [143], [145]-[147]. The SVET is capable of resolving locally-occurring corrosion events due to its use of a microtip electrode which can be moved in three dimensions.

When a current source is present in solution, a potential gradient is produced outwardly from the source. The SVET resolves this in to an alternating potential at the vibration frequency and in the direction of vibration. The magnitude of the alternating potential is 
proportional to the normal component of the ionic current flux passing through the solution [145].

Corrosion studies were possible as local anodic and cathodic events occurring on a metallic surface can be considered to be point current sources. Thus, the SVET can be utilized to examine the corrosion behavior of metallic materials by uncovering the location and intensity of these events as they are occurring on/above the metallic surface. Time-dependent metal loss and anodic/cathodic current density can be semi-quantified which allows for comparison between competing metal systems, coatings and electrolyte additions.

Study of battery electrodes in alkaline conditions had not been performed previously with the SVET. It is important to verify the accuracy of the SVET in these conditions against a simple and well understood technique.

\subsubsection{Sample Preparation}

To ensure uniformity and repeatability between tests, a certain amount of sample preparation was required.

\section{Polished planar samples}

SVET tests were conducted using $1 \mathrm{~mm}$ thick $25 \mathrm{~mm}$ disks of Fe $99.5 \%$ supplied by Goodfellow Cambridge Ltd. Samples were polished to $1 \mu \mathrm{m}$ and degreased with ethanol and air-dried immediately prior to testing. During testing, $10 \mathrm{~mm}^{2}$ of the surface of the polished iron was exposed to solution through use of the sample holder described in section 5.2. Polished samples were used to reduce potential variables and iron was used as it was the active material in the iron electrode.

\section{Battery electrodes}

Non planar iron electrodes (5.5) were cleaned with ethanol and air-dried immediately prior to testing. Polishing was not performed as not to disturb surface morphology. 


\subsubsection{SVET Apparatus}

During testing, a glass-encased $125 \mu \mathrm{m}$ platinum wire was scanned across the surface of the sample at a constant scan height of $100 \mu \mathrm{m}$. The platinum wire was encased so that only the $125 \mu \mathrm{m}$ cross section was exposed to the electrolyte and sample surface. A schematic of the SVET probe and which is vibrated using a speaker is shown in Figure 19. This researcher found probes had to be remanufactured frequently due to breakages, possibly exacerbated by the aggressive electrolyte being used. The SVET probe was scanned over the surface to be investigated every 15 minutes for a total of 15 hours (60 scans) for each experiment. This data was used to produce a $15 \mathrm{~h}$ representation of the surface activity [145]. Throughout all experiments it is assumed that the dissolved oxygen concentration in the bulk solution was constant at $2.8 \times 10^{-4} \mathrm{~mol} \mathrm{dm}^{-3}$ - the equilibrium concentration for air-saturated water [179].

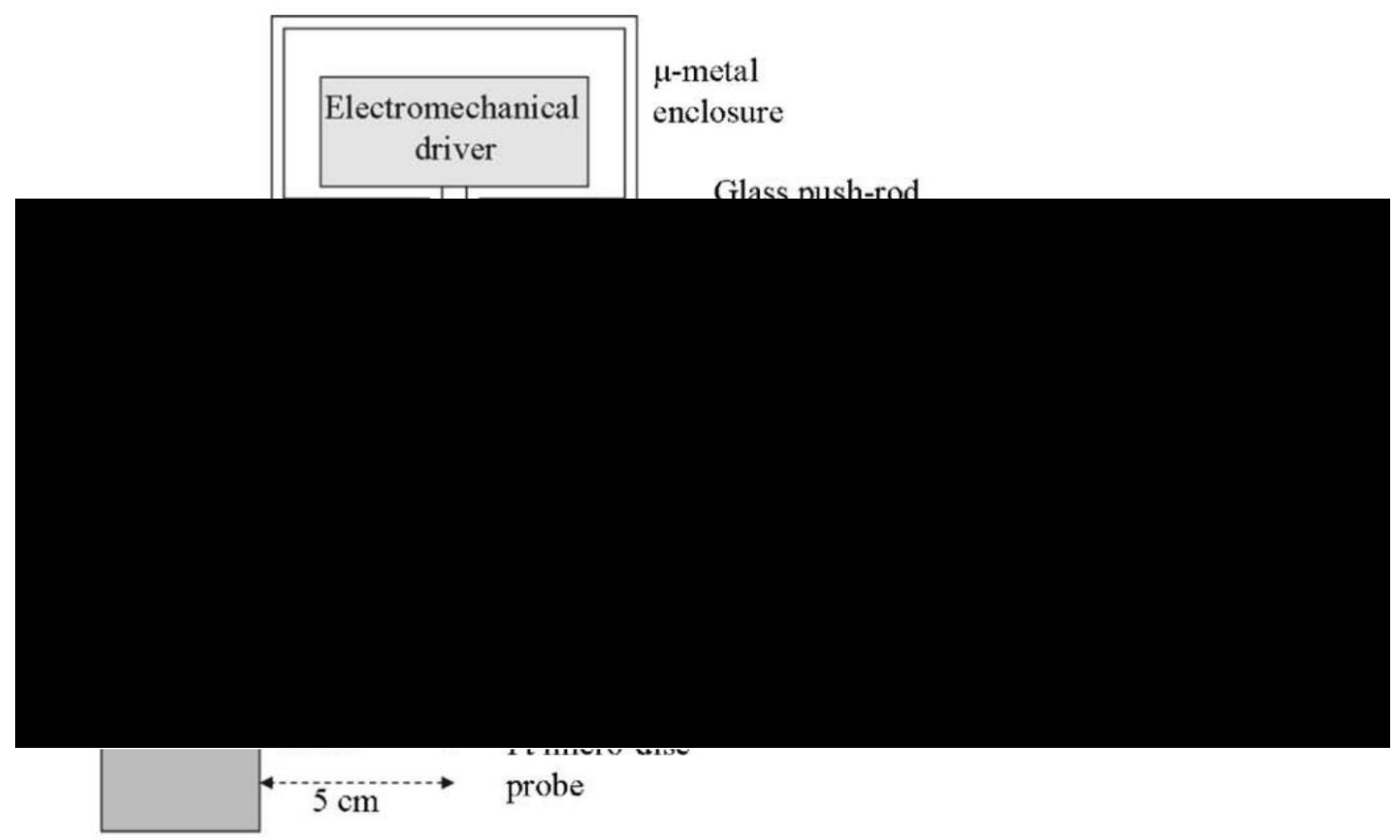

Large, low impedance $\mathrm{AgCl}$ reference electrode (instrument earth)

Figure 19 Schematic of the SVET [155] 
During all experimentation, the sample under investigation was held horizontally in solution, normal to the vertically orientated vibrating probe. This was a requirement for use of the SVET, however the author is conscious this may have slowed the reaction rate when compared to an equivalent electrochemical cell with the working electrode held vertically. This theory is based on the assumption that, when held vertically, bubbles of gas will be removed by collision with other bubbles during gas evolution. This will act to 'clear' the surface of bubbles, having the effect of increasing the available electrode surface for reaction. Conversely, when the electrode is held horizontally, evolution of a single bubble does not remove any additional bubbles and areas of the surface with adhered gas bubbles cannot sustain electrochemical reactions with the electrolyte. Regardless, this researcher will consistently arrange the working electrode horizontally in the cell and therefore, avoid any variation which could be caused by this effect.

\subsubsection{Surface Profiling}

Prior to commencing experimentation, surface profiling was required. Three methods were possible: manual levelling, interpolated height scanning and 3D height scanning. All planar samples were examined using manual levelling and all non-planar (contoured/rough) samples were examined using 3D height scanning. Accordingly, interpolated height scans are not discussed in this body of work.

\section{Manual levelling}

In this method, the sample was adjusted until the profile was normal with respect to the SVET probe across the entire scan area, prior to commencement of the SVET experiment. This was achieved through positioning of the SVET probe in the corner of the sample and manipulating it until it was touching the surface of the sample. This was used as a datum and the screws on the levelling stage (5.2.4) were adjusted until all four corners and thus the entire planar sample, were level and normal/horizontal with respect to the SVET probe. The experiment was then commenced, with the sample level and the SVET probe the desired height above the surface of the entirety of the sample. It was assumed that the sample is flat, however this is not inappropriate for a polished sample. 
This technique was not suitable for non-planar samples, so 3D height scanning was used.

\section{D height scan}

Samples which were non-planar, or had undergone surface treatment such as mechanical abrasion and were not suitable for polishing, were examined as received. In order for the SVET probe to remain the desired height above the sample, it was necessary to produce a full 3D height map for the entirety of the scan area, before the experiment could commence.

3D height scanning utilizes an automated program, which compares the signal between the SVET probe speaker (typically used for probe vibration) and a second, identical and isolated reference speaker. It is possible to detect subtle perturbations, such as the surface of the sample. The lock-in amplifier compares the signals from both speakers and is able to detect the 'deformation' in the signal as the mechanically attached probe impacts the sample surface. 3D height scanning uses a higher oscillation frequency $(900 \mathrm{~Hz})$ to ensure a probe vibration amplitude of $<1 \mu \mathrm{m}$. A minimum deformation threshold is used to ensure that false surface readings do not occur. The exact mechanism for surface detection is described elsewhere [180].

The software moves the probe through every single data scan point and logs the height in which the probe impacts the surface of the sample. This results in height information for each point of the data scan. This data is written to memory and used to determine the height at which the probe should be set when recording the signal from each point across the sample surface.

\subsubsection{Operation of the SVET}

A speaker was used to vibrate the $125 \mu \mathrm{m}$ platinum SVET probe normal to the sample surface at $140 \mathrm{~Hz}$ and at an amplitude of $30 \mu \mathrm{m}$. The amplitude of the vibration of the tip was measured using a stroboscope light under a microscope to allow easier measurement. 
The probe speaker frequency control and probe signal measurements were carried out using a Perkin Elmer EG\&G 7625 Lock-in Amplifier. The signal-to-noise ratio was improved by digital signal averaging of ten successive measurements.

Movement of the probe in 3 dimensions over the sample surface was possible due to use of a stepper motor-driven tri-axial micromanipulator platform, manufactured by Time and Precision Ltd.

The $125 \mu \mathrm{m}$ glass-encased platinum probe was vibrated, as described above, by attachment to the cone of a speaker via a glass push rod and a machined Teflon ${ }^{\circledR}$ sheath/holder. The length of the probe/push rod assembly was limited as far as is practicable to reduce the effect of any bending forces and ensure that the probe remained normal to the sample. Both the speaker and the push rod are encased in a Mu-metal box in order to shield the probe and associated wiring from electromagnetic leakage associated with the speaker.

\subsubsection{Calibration of the SVET}

The SVET measures the normal component of the current flux generated by a current source in solution. This is possible due to the vibration of the platinum microtip electrode above the point current source at a constant height, frequency and vibration amplitude. The potential measured by the SVET is directly proportional to the magnitude of the electrical field, F, or the potential gradient in the direction of vibration, Equation 2.2-1:

$$
F=\frac{d E}{d z}=\frac{i z}{2 \pi \kappa\left(x^{2}+y^{2}+z^{2}\right)^{1.5}}
$$

Where, $E$ is the electrical potential and $z$ is the direction of vibration. When a point current source (i) is considered, the field strength can be calculated at a distance (x,y,z) from the point source in a medium of conductivity $(\kappa)$ [181].

Maximum field strength ( $\mathrm{F}_{\mathrm{Max}}$ ) occurs when $\mathrm{x}$ and $\mathrm{y}$ are 0 - when the probe is directly over the point current source, as shown in Equation 2.2-2:

$$
F_{\text {Max }}=-\frac{i}{2 \pi \kappa z^{2}}
$$


Where $i$ is a point current source, $\kappa$ a medium of conductivity and $z$ is the direction of vibration.

Ohm's law relates the current flux density along the axis of probe vibration $\left(j_{z}\right)$ to the peak-to-peak SVET voltage signal (Vpp), as shown in Equation 2.2-3:

$$
V_{p p}=J_{z}\left(\frac{A_{p p}}{\kappa}\right)
$$

A SVET calibration factor $(\mathrm{G})$ can be defined using Equation 2.2-4 in a medium of conductivity $(\kappa)$ :

$$
G=\frac{\kappa}{A_{p p}}
$$

Where $A_{p p}$ is the peak-to-peak vibration amplitude, which can be measured using a strobe light combined with a travelling microscope. However, this measurement is difficult to obtain with sufficient accuracy, so an alternative method is favored for calibration which employs a two-compartment cell.

\section{Two compartment tube cell calibration}

A glass cell with a tube of known diameter is used to produce a current flux of known density, as illustrated in Figure 20. 


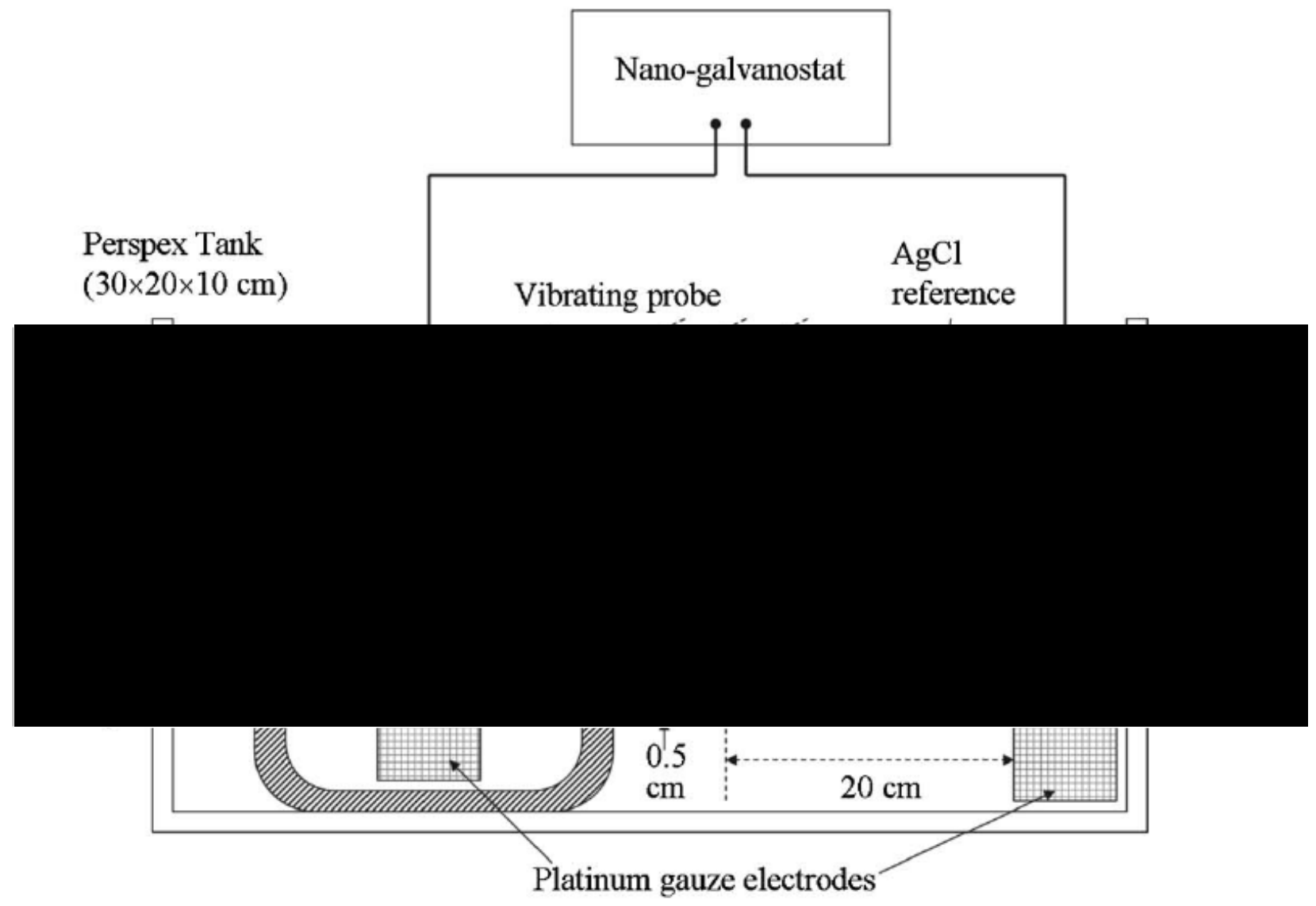

Figure 20 Two compartment tube cell [155]

A nano-galvanostat was used to pass a range of currents through a tube via two $10 \mathrm{~mm}^{2}$ platinum gauze electrodes. The compartments were linked solely by a vertical tube of 60 $\mathrm{mm}$ height and $5 \mathrm{~mm}$ internal diameter and the chosen electrolyte was added to both compartments. During calibration, the probe was manipulated such that it was inside the center of the tube. It is assumed that the current flux density was constant and uniform across the diameter of the tube - thus equal to the current passed through the cell divided by the cross-sectional area of the tube (excluding the cross-sectional area of the SVET probe). It is further assumed that the current flux was aligned vertically - both parallel with the tube axis and the probe vibration axis. 


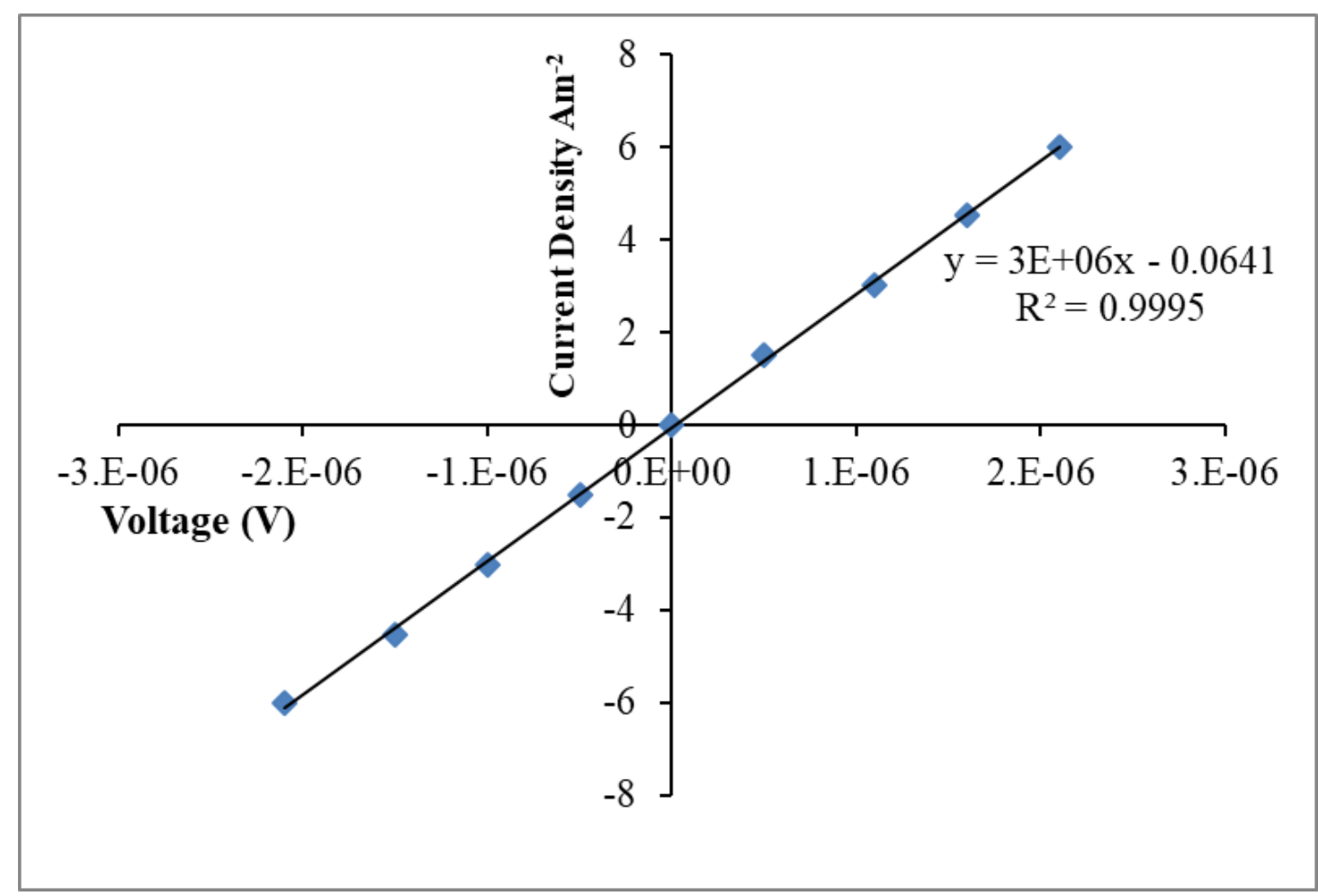

Figure 21 Typical calibration plot [152]

The voltage detected by the SVET was measured for each resultant current density and a linear relationship was observed as shown in Figure 21. The gradient from this relationship is used as a calibration factor for the instrument. Calibration was repeated before each experiment.

\subsubsection{SVET Data Analysis and Visualization}

\section{Individual scans}

Data recorded by the SVET during scanning is expressed in $\mathrm{nV}$. Multiplying by the calibration factor for the experiment converts the data to $\mathrm{Am}^{-2}$, which can be visualized using the cartography software Surfer® 10 (Golden Software). False color contour maps (iso-current density maps) were created to show anodic (red) and cathodic (blue) data. Signal strength was expressed by the intensity of the colors with respect to the scale bar. 


\section{Multiple scans}

Semi-quantitative analysis of data was possible through integration of the SVET-derived current density $\left(\mathrm{j}_{\mathrm{z}}\right)$ data using Equation 2.2-5. It was assumed that the corrosion activity remained constant over the period between the scans. Typically, for corrosion studies, this integration is performed on the positive (anodic) voltage as described elsewhere [148]. This data can be used to approximate the anodic metal dissolution, allowing for comparison between different metal systems. Area-averaged anodic current was calculated by integrating the area $(x, y)$ under the positive (anodic) voltages recorded by the SVET using Surfer ${ }^{\circledR} 10$ (Golden Software), after conversion to $\mathrm{Am}^{2}$ through multiplication with the calibration factor as described previously.

$$
i_{a t=\int_{0}^{x} \int_{0}^{y}\left[j_{z(x, y)}>0\right]}
$$

Anodic charge can be calculated from the area-averaged anodic current $\left(\mathrm{i}_{\mathrm{at}}\right)$ data using Equation 2.2-6 on the assumption that electrochemical activity remains constant between the 15 minutely scans.

$$
Q_{a t}=i_{a t} t
$$

Where $Q_{a t}$ is charge and $t$ is time (900 seconds).

Faraday's $2^{\text {nd }}$ law of electrolysis (Equation 2.2-9) can then be used to determine mass loss for each SVET scan and over the period of the experiment by summing this data. In addition, location and intensity of anodic events can also be examined [152].

In experiments where a strong cathodic bias voltage was applied and cathodic data is of interest, it was possible to use Faraday's Law in a similar manner to approximate moles of hydrogen evolved, as this was the primary reaction occurring (see Figure 72). As previously, area-averaged cathodic current $\left(i_{\mathrm{ct}}\right)$ was calculated by integrating the negative (cathodic) SVET-derived current density $\left(\mathrm{j}_{\mathrm{z}}\right)$ area $(\mathrm{x}, \mathrm{y})$ data, as per Equation 2.2-7:

$$
i_{c t=\int_{0}^{x} \int_{0}^{y}\left[0>j_{z(x, y)}\right]}
$$


As in the case of anodic analysis, cathodic charge $\left(\mathrm{Q}_{\mathrm{ct}}\right)$ can be calculated from the area-averaged cathodic current $\left(\mathrm{i}_{\mathrm{ct}}\right.$ ) data using Equation 2.2-8 on the assumption that electrochemical activity remains constant between the 15 minute scans (where $t$ is time):

$$
Q_{c t}=i_{c t} t
$$

Faraday's $2^{\text {nd }}$ law of electrolysis (Equation 2.2-9) is used to convert cathode-derived charge $\left(\mathrm{Q}_{\mathrm{ct}}\right)$ to the number of moles $(\mathrm{N})$ of hydrogen on the assumption that all charge passed results in hydrogen gas being evolved:

$$
N=\frac{Q_{c t}}{n F}
$$

In which $F$ is Faraday's constant and $n$ is the number of electrons passed in the reaction (two for evolution of a hydrogen molecule). This data is typically expressed as the number of moles of $\mathrm{H}_{2}$ evolved per $\mathrm{m}^{-2}$ of sample area.

\subsubsection{Limitations of the SVET}

SVET is a powerful and complex technique and as such, certain assumptions must be made during operation and data analysis, which result in several key limitations.

1. It was assumed that the surface activity remains constant over the period between scans, that the snapshot recorded in the scan data is valid until the following scan takes place. This may result in minor inaccuracies if there are significant changes in sample behavior which take place over a small time frame - the SVET may fail to record this information. Scan time was reduced as far as practical however, one scan takes 11-14 minutes to complete, so scans more often than every 15 minutes were not practically possible.

2. The SVET is only capable of detecting the signal when the probe travels through the current flux. The plane of the scan $(100 \mu \mathrm{m}$ perpendicular to the surface) must coincide with charged species in the electrolyte in order for them to be detected. In a corrosion context, this limits the SVET to the measurement of localized corrosion and not general corrosion [150]. 
3. Resolution. Features not separated by a distance greater than the SVET resolution will not be detected. This is considered an advantage of the SVET (vs. other techniques) due to the low height of the Pt microtip during scanning [182].

For these reasons, the SVET is described only as semi-quantitative. Trends of the surface electrochemical activity are recorded, but this should not be assumed to be the same as the actual values. The advantage in use of SVET for analysis of battery electrodes is that it can be used to identify and locate local heterogeneity in the surface of the material.

\subsection{Gas Collection Testing}

Gas collection experiments were used to verify the results of the SVET testing. This is a simple and well understood technique, which is described in detail by Song et al. [183][185].

A sample immersed in electrolyte was held horizontally using the sample holder and stage set up described previously (5.2.3 - 5.2.4). An upturned burette $(50 \mathrm{ml} \pm 0.050 \mathrm{ml})$ was placed vertically, with the opening located directly above the sample at a height of $10 \mathrm{~mm}$. The burette was filled with the test electrolyte and hydrogen gas evolved from the sample was collected during testing. The sample was cathodically polarized to $-1.4 \mathrm{~V}$ vs. $\mathrm{Hg} / \mathrm{HgO}$ (CH152 IJ Cambria Scientific Ltd) using a Solartron SL 1286. The volume of this gas was recorded at 15 minute intervals and the number of moles of hydrogen evolved was calculated.

\subsubsection{Sample Preparation}

Sample preparation was identical to SVET sample preparation for polished planar samples, as described in 2.2.1. This was required to allow comparison between the analytical techniques. 


\subsubsection{Equipment Setup}

A burette (Fisher Scientific) was upturned above the sample and filled with the test electrolyte (Figure 22). Approximately $1 \mathrm{~L}$ of electrolyte was used during each test. The $\mathrm{Hg} / \mathrm{HgO}$ reference $\mathrm{Hg} / \mathrm{HgO}$ (CH152 IJ Cambria Scientific Ltd) was placed adjacent to the sample, with a $20 \mathrm{~mm}^{2}$ platinum gauze acting as a counter electrode.

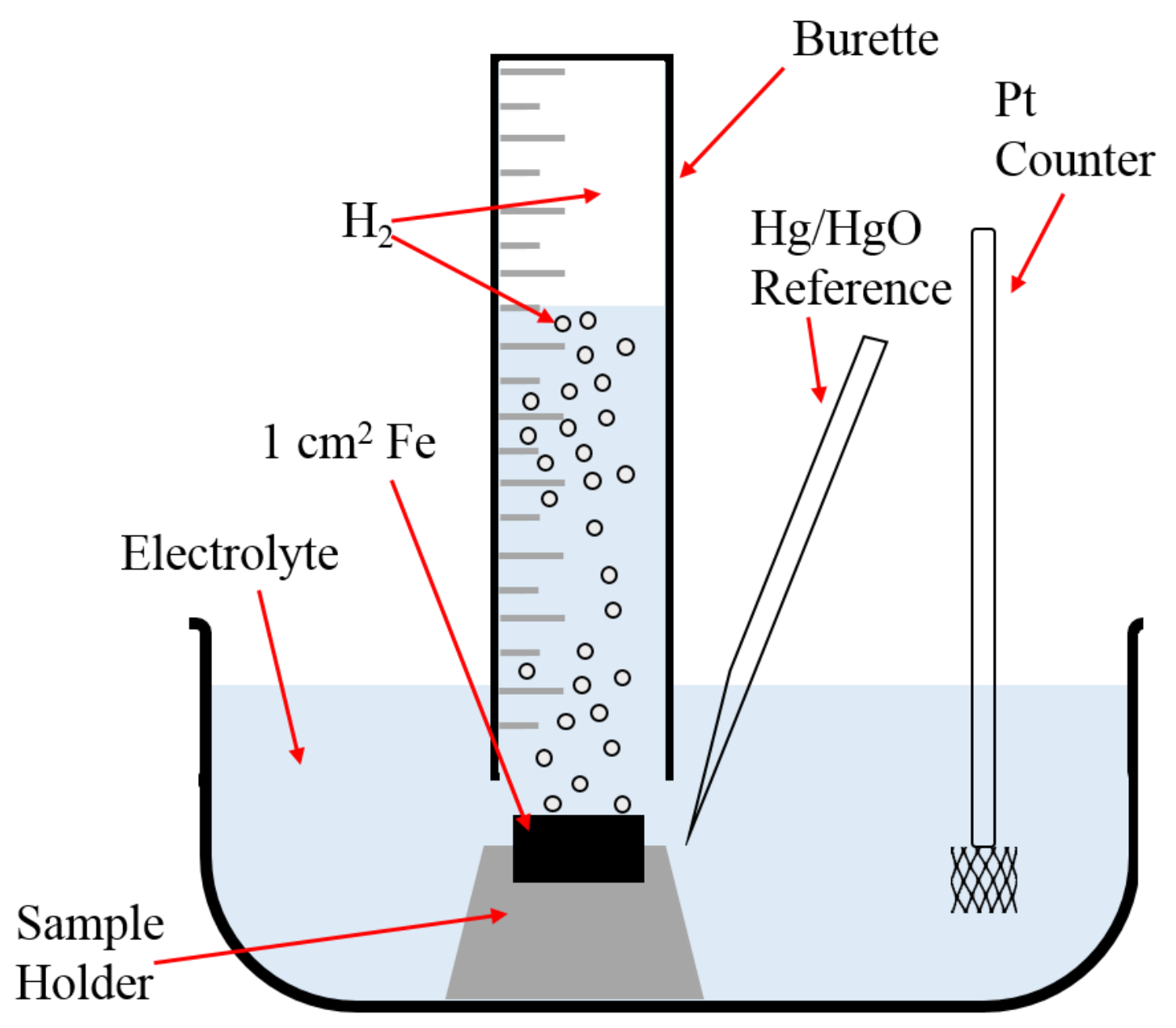

Figure 22 Gas collection cell setup

The Solartron SL 1286 was set to record current data at one second intervals whilst cathodically polarizing the sample to $-1.4 \mathrm{~V}$ vs. $\mathrm{Hg} / \mathrm{HgO}$ (CH152 IJ Cambria Scientific Ltd). The reading from the burette was recorded every 15 minutes. The working electrode was held horizontally, as discussed previously (2.2.2). 


\subsubsection{Data Analysis}

The volume $\left(\mathrm{cm}^{3}\right)$ of hydrogen gas evolved in to the burette was recorded every 15 minutes for 15 hours. The ideal gas law was used to calculate the molar volume (V) of a gas at room temperature and pressure (Equation 2.3-1 to 2.3-3):

$$
\begin{gathered}
P V=n R T \\
V=\frac{n R T}{P} \\
V=\frac{1 \times 0.082057338 \times 293.15}{1}
\end{gathered}
$$

Where $R$ is the gas constant $\left(\mathrm{L} \mathrm{atm} \mathrm{K}^{-1} \mathrm{~mol}^{-1}\right), T$ is room temperature $(\mathrm{K}), P$ is pressure (atm) and $n$ is the number of moles.

The resulting molar volume was used to calculate the moles of gas which had been evolved at each 15 -minute interval. This data was typically expressed as number of moles of $\mathrm{H}_{2}$ per $\mathrm{m}^{-2}$ of sample area.

\subsubsection{Limitations of the Gas collection testing}

Whilst this technique is well understood, there are a few obvious limitations.

It was assumed that the electrolyte expelled from the burette imparts no pressure on the hydrogen present in the burette, in reality the expelled fluid will impart a negligible amount of pressure to the gas inside the burette.

It was assumed that the hydrogen evolved during the experiment acts as an ideal gas and thus that the ideal gas law is applicable.

It was assumed that only hydrogen gas is collected in the burette. Gasses dissolved in the solution are not considered. Gas which does not enter the burette was not considered.

More accurate gas measurements could have been completed using a manometer to measure pressure. 


\subsection{Scanning Kelvin Probe (SKP) for Iron Electrode Investigation}

The SKP is utilized in 5.5 for the investigation of the level of atomic hydrogen present in an iron foil. This is possible due to the interaction of the hydrogen with surface iron oxides following charging on the reverse face. Measurable reductions in Volta potential occur when atomic hydrogen interacts with the air-formed surface oxide film due to the effect of hydrogen on the ratio of $\mathrm{Fe}^{\mathrm{II}} / \mathrm{Fe}^{\mathrm{III}}[173]-[175]$, [178]. Prior to scanning with the SKP, the sample was cathodically impregnated with hydrogen on the reverse face through potentiostatic charging for various time periods. SKP has been used to observe potentials in a variety of conditions.

\subsubsection{Sample Preparation and Pre-treatment}

\section{Sample Preparation}

Iron foil samples of $0.1 \mathrm{~mm}$ thickness and $99.9 \%$ purity were supplied by Goodfellow Cambridge Ltd. These samples were then degreased on both faces prior to pre-treatment. The sample holder as described in 5.2.3 was modified such that a $5 \mathrm{~mm}$ diameter opening was available.

Preliminary testing showed poor repeatability when samples were abrasively cleaned prior to testing or degreased with more aggressive solvents, accordingly, ethanol was used to clean samples in this work.

\section{Sample Pre-treatment}

Pre-treatment was carried out for various time lengths in solutions of $0.2 \mathrm{M}$ potassium hydroxide, with additions of $0.1 \mathrm{M}$ thiourea or $0.1 \mathrm{M}$ benzotriazole. During this pretreatment, a Solartron SL 1286 was used to hold the samples at $-1.4 \mathrm{~V}$ vs. $\mathrm{Hg} / \mathrm{HgO}$ (CH152 IJ Cambria Scientific Ltd) with a $20 \mathrm{~mm}^{2}$ platinum gauze acting as a counter electrode. Immediately after pre-treatment, samples were rinsed with distilled water prior to SKP analysis of the reverse face, as shown in Figure 23. 


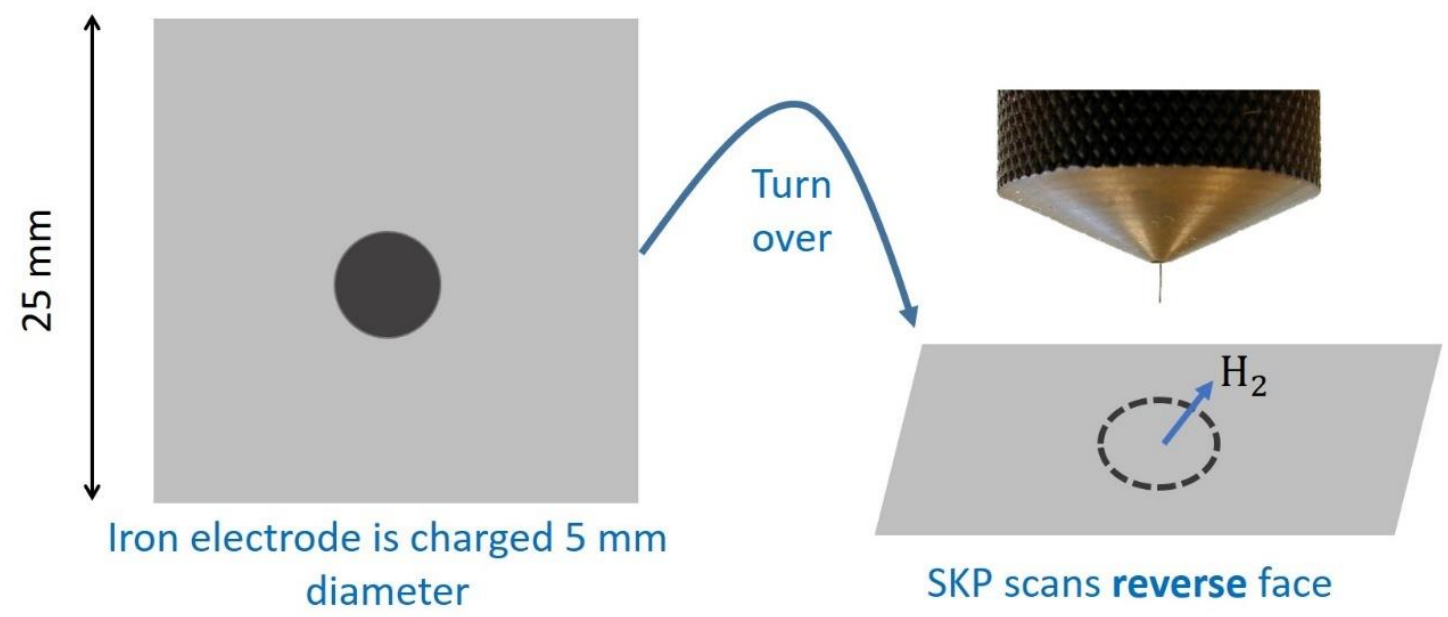

Figure 23 Schematic of SKP sample

\subsubsection{SKP Apparatus}

The SKP employs a computer-controlled three-dimensional micro manipulator stage and a vibrating gold reference probe, all housed within a stainless steel environmental chamber (Figure 24). As the probe was manipulated, data was recorded on the computer. As reported elsewhere [170], the tip of the vibrating probe and the stainless steel environmental chamber were held at earth potential. All ancillary equipment (drive electronics and speaker) were positioned outside the stainless steel environmental chamber for purposes of electrostatic and magnetic shielding of the reference probe and sample. 


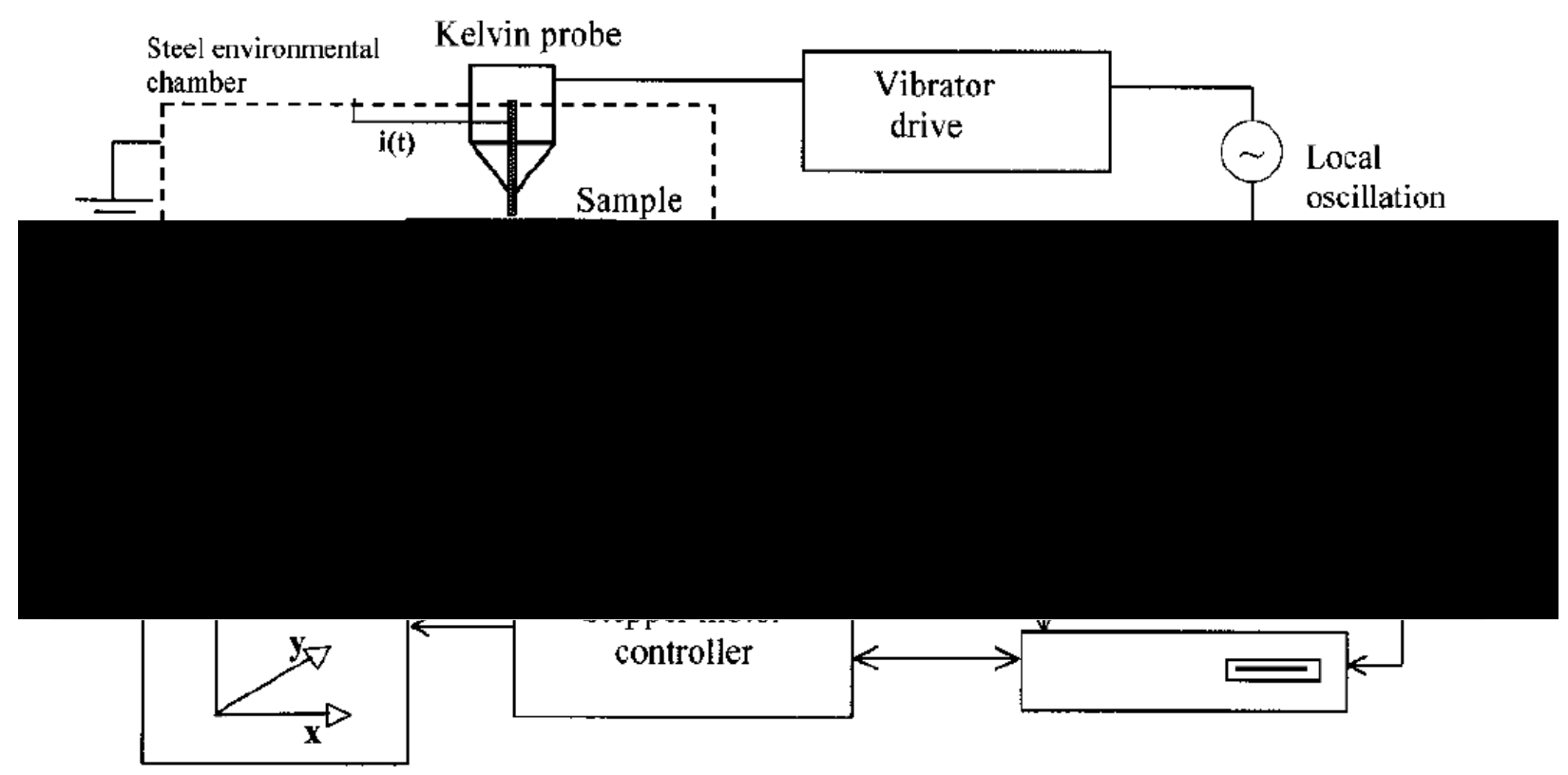

Figure 24 Schematic of SKP apparatus [164]

The SKP reference probe consisted of a straight $125 \mu \mathrm{m}$ gold wire (99.99\% purity, Goodfellow Cambridge Ltd.) vibrated above the surface of the sample in the normal direction. The reference probe was connected to a speaker via a glass push rod. The speaker was driven at $280 \mathrm{~Hz}$ by a Perkin Elmer EG\&G 7625 Lock-in Amplifier using the oscillator function. A probe amplitude of $50 \mu \mathrm{m}$ was used - measured using a stroboscope light under a microscope.

During operation, an alternating current was generated due to the periodic variation in capacitance between the sample and the reference probe. This signal was converted into an ac voltage by a dc biased transimpedance amplifier circuit. The lock-in amplifier detected this signal and the corresponding dc bias voltage output was supplied to the sample via an integrator-based feedback system in order to automatically null it. The feedback loop acts to nullify the alternating current by equalizing the Galvani potentials of the probe and the specimen, by altering the dc bias voltage until the system reaches null conditions. In this instance, the magnitude of the reverse of the bias voltage is equal to the local Volta potential difference existing between the reference probe and the sample. The digitized dc bias voltage $\left(E_{k p}\right)$ values were recorded by the attached computer. 


\subsubsection{Operation of the SKP}

After pre-treatment (2.4.1), the sample was mounted on the levelling stage and manually levelled. This process required the positioning of the probe over one corner of the sample and manipulating the sample height to the point where the reference probe contacted the sample. At this point the sample was lowered to a distance of $100 \mu \mathrm{m}$ above the probe and the process was repeated on the opposite corner of the sample. After adjustment of the sample stage, the sample was positioned such that the reference probe could be scanned over the surface at a constant height of $100 \mu \mathrm{m}$. Henceforth, $100 \mu \mathrm{m}$ was used as the scanning height during all experimentation. This operation is similar to the manual leveling of the SVET stage discussed previously.

All SKP measurements were carried out at $25{ }^{\circ} \mathrm{C}$. Humidity of the SKP chamber was controlled to $95 \% \mathrm{RH}$ through use of reservoirs of electrolyte. These were placed in the SKP chamber after levelling and the chamber was sealed. Humidity control is particularly important in this testing [173].

Both 2D surface maps and 1D line scans were recorded in this study. 1D line scans were recorded from one side of the sample to the other side, directly across the reverse of the area of hydrogen evolution. In the line scans, the drop in the kelvin potential from the unexposed area to the reverse of the area exposed to cathodic hydrogen evolution was extrapolated from the data (described below). 2D surface maps were also recorded at hourly intervals immediately following cathodic hydrogen evolution pre-treatment. These data were recorded in a grid format relating to the $2 \mathrm{D}$ positioning of each kelvin potential recording.

\subsubsection{SKP Data analysis and visualization}

\section{D Maps}

Maps of the $E_{\text {corr }}$ vs. distance data for a scan of the sample area were produced using Surfer ${ }^{\circledR} 10$ (Golden Software). A scale bar was added to ensure data from different samples was comparable. 


\section{D line scans}

$\mathrm{E}_{\text {corr }}$ vs. distance data was viewed in Microsoft Excel. The change in Volta potential from the substrate, to the reverse of the cathodic hydrogen evolution area was then calculated for each sample. This is illustrated in Figure 25. This measurement is referred to as 'Volta potential drop' throughout this document.

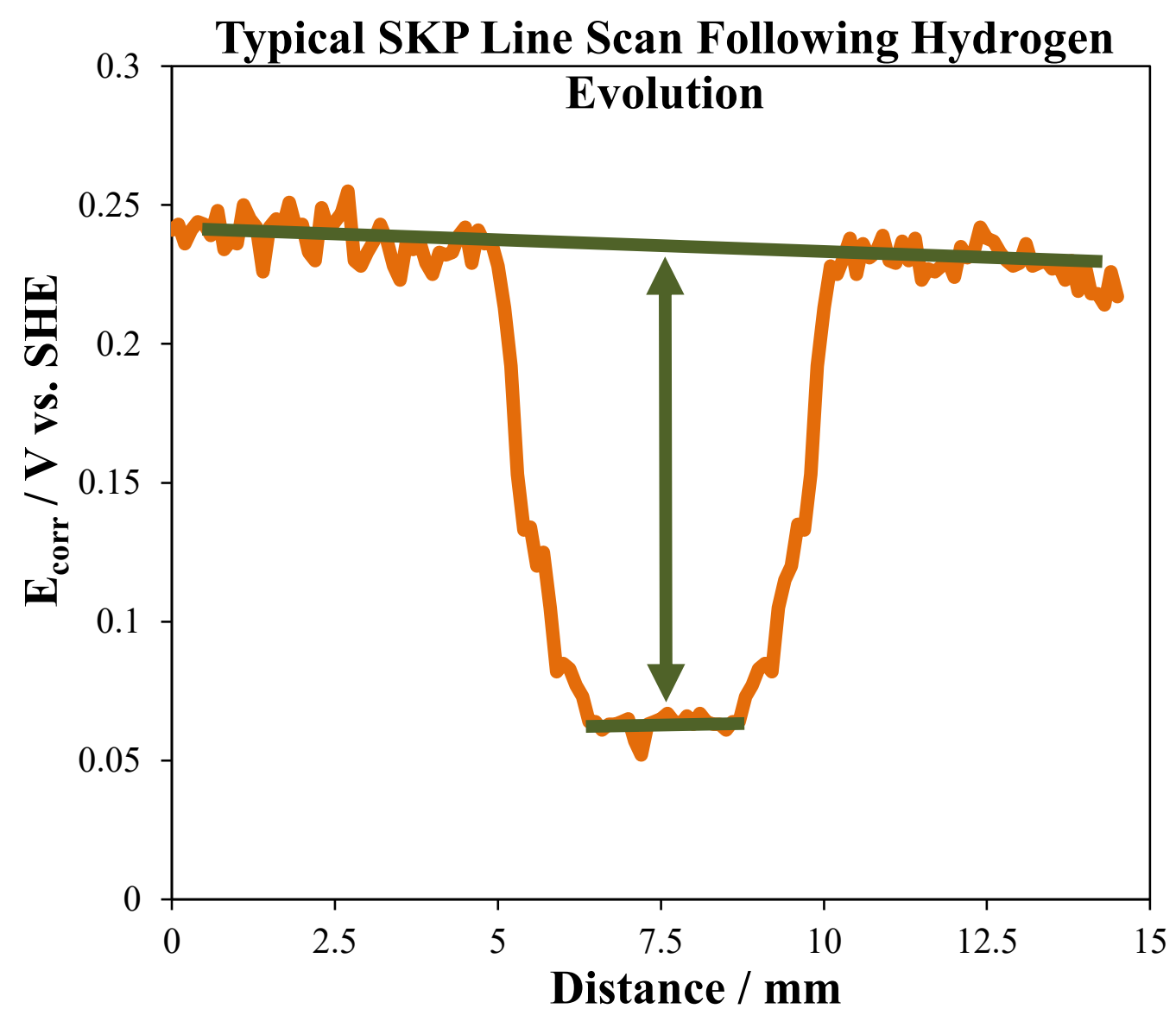

Figure 25 SKP-derived line scan across the reverse of the hydrogen evolution

region

\subsection{White Light Interferometry (WLI)}

Surface topography of electrodes and electrode substrates was examined using White Light Interferometry. This non-contact technique can identify surface roughness and features present on the sample. 


\subsubsection{WLI Apparatus}

A Veeco Wyko NT9300 was used for all measurements. The instrument was controlled by a personal computer running Wyko Vision32 version 2.210. The NT9300 was suspended on an air table to minimize the effect of vibrations within the laboratory.

This apparatus is capable of measurements using both phase shifting interferometry (PSI) and vertical scanning interferometry (VSI). PSI uses a monochromatic red light beam and is typically used on highly mirrored/reflective (smooth) surfaces with a working measurement range of $160 \mathrm{~nm}$ - therefor this technique is not suitable for looking at electrode substrates or iron electrodes [191]. VSI uses a white light source with a working measurement range of $500 \mu \mathrm{m}$. All measurements in this work were carried out using VSI.

\subsubsection{Operation of WLI}

Interference patterns between a reference beam and a working beam are used to examine a sample. The beam is generated, travels through various lenses and is split into working and reference beams using a beam splitter. The reference beam is reflected using a reference mirror and returned to the beam splitter (Figure 26). The working beam is directed at the sample and also reflects to the beam splitter, thus both beams are recombined. This forms an interference pattern (fringes), which occur due to the differences in the waveforms of the reflected beams. 


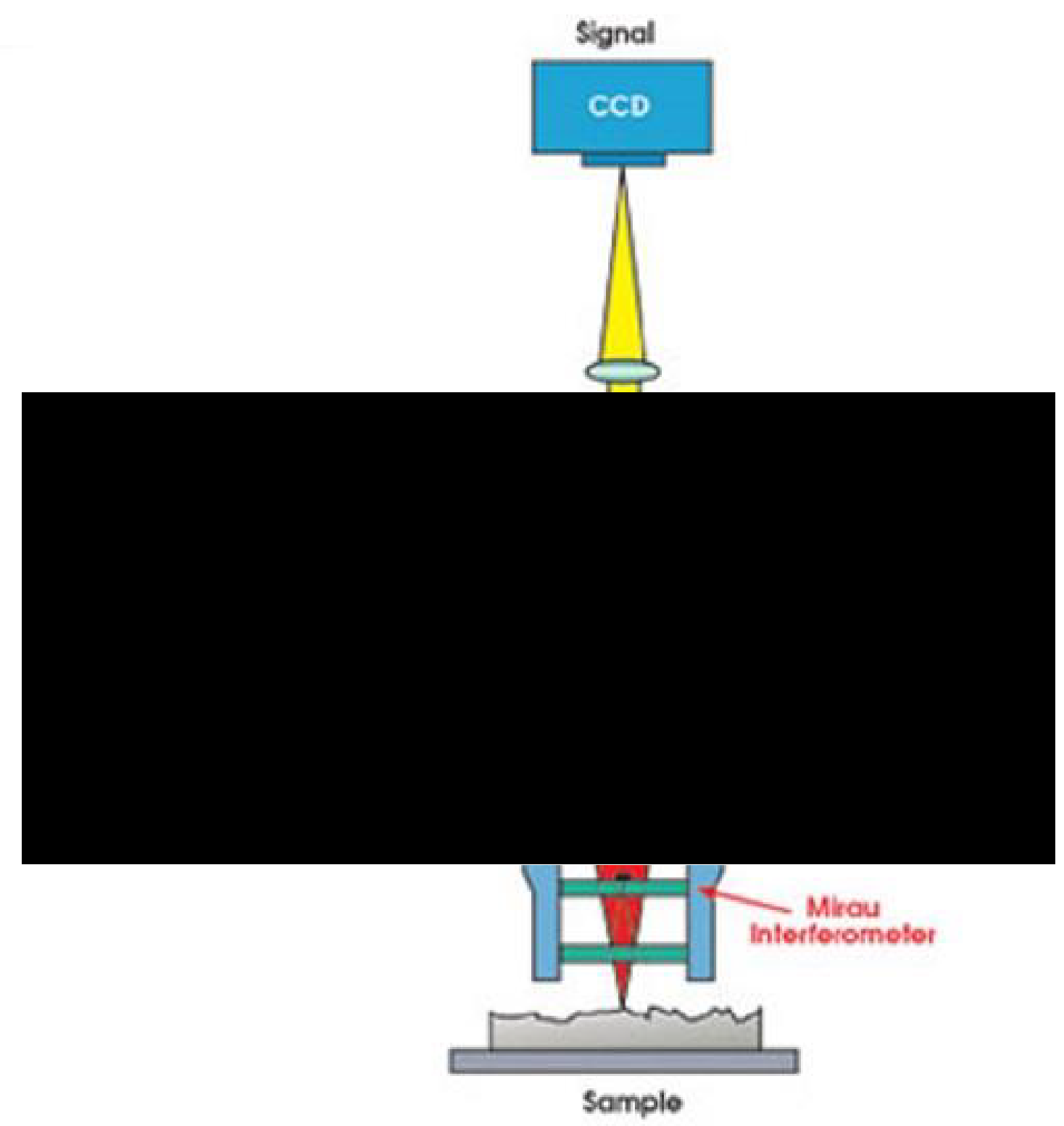

Figure 26 Schematic of a white light interferometry apparatus (adapted from [192])

The system must be focused for this interference pattern to be useful. By the nature of the technique, samples are not perfectly flat, therefore, the focus must be adjusted during scanning to attain data for the sample's contours. During measurements, the lens and thus the focus is scanned vertically in order to provide the interference patterns for all contours of the sample. 


\subsubsection{Limitations of White Light Interferometry}

When utilizing VSI, information can only be gathered when the system is focused on the sample. For this reason, care was taken to ensure all contours of the sample were included in the measurements. It is possible that taller features extended above the focal range and smaller crevices were below the focal range.

Certain features, such as crevices, were particularly prone to not returning sufficient light for interference patterns to be analyzed. In this instance, the threshold required for analysis could have been lowered, however this may have resulted in ambient light and other undesirable reflections from entering the lens. This (reduced threshold) was limited where possible, however, black areas appear in images where no contour data is recorded. During analysis, care was taken to avoid reading too much in to these areas where analysis was not possible.

\subsection{Other Techniques}

Techniques used for gaining additional information on the materials discussed in this thesis are included below.

\subsubsection{Thermogravimetric analysis (TGA) and Derivative Thermogravimetry (DTG)}

Thermogravimetric analysis is a technique where a sample is heated from one temperature to another temperature and the weight of the sample is measured continuously. This can be used to assess the reactions which are occurring during heating. It is a useful technique in the context of electrode manufacture as it enabled this researcher to gain an understanding of the solvents and resultant key temperatures associated with the iron inks used in this study.

A Perkin Elmer STA6000 was used for TGA experimentation. Whilst controlled atmospheres could have been used, air was used to ensure the decomposition of the ink was representative of what would occur on a hot plate or in a NIR furnace. 
Derivation of the TGA data was also performed to attain Derivative Thermogravimetry (DTG) values to understand the rate of change of the mass of the sample. This was calculated using 10 point moving averages and exponential smoothing with a damping factor of 0.9 in Microsoft Excel.

\subsubsection{Scanning Electron Microscopy (SEM)}

This common analytical technique was used to investigate the resulting material produced when heating the iron inks. A Hitachi TM3000 desktop microscope was used to image various coupons. This method employs an accelerated electron beam and various detectors to form an image from the resulting backscattered or secondary electrons. It was of use to the researcher as different materials can be easily identified due to the way they interact with the electron beam and the contrast in the resultant image.

Energy-dispersive X-ray spectroscopy (EDS) was used to enhance understanding of the materials present in the sample. Through the interaction of the electron beam with the atoms in the sample, X-rays are produced. The energy of the X-rays is characteristic of the atom from which it was produced. Accordingly, the Buker Quantax70 EDS apparatus (which was integrated into the Hitachi SEM) was capable of identifying the elemental composition of the sample with sufficient accuracy for the atomic weight range of materials used in this study. 


\section{Chapter 3: Printed Iron Electrodes for Low Cost Alkaline Batteries - Low Temperature Route}

\subsection{Introduction}

Current commercial manufacturing techniques of iron electrodes utilize 'pocket plate' construction and are labor intensive and time consuming to produce [26]. This complex production process (1.6.4) is said to result in a product which is eight times more expensive than its raw material [53].

A method of production of iron electrodes involving printing of an ink of active material would allow for electrodes to be produced on a continuous production line. State of the art rapid processing routes were investigated here, which allow for curing/sintering in seconds, as opposed to minutes and are essential for enabling roll-to-roll manufacture of large-scale electrodes. Additionally, updated iron electrodes could have an improved specific capacity at a reduced cost [53].

Other work [81] has shown that inclusion of additives during electrode manufacture can lead to significant improvements in performance. Additives, such as inhibitors, can easily be included to inks prior to printing of electrodes.

High-purity carbonyl iron (produced the by decomposition of iron pentacarbonyl) was used exclusively as the source of active material for this research due to successful results reported elsewhere [22], [80], [193]. Impurities, such as carbides and nitrides, are capable of promoting self-discharge and some metal impurities are soluble in the alkaline electrolyte [135].

\subsubsection{Printed Electrodes}

Printing is a versatile method of manufacture, with applications ranging from production of photovoltaics, heating elements and electronics [194]. Modern battery manufacturers use printing/coating methods to produce the majority of products [34], [195]. More recently, printing has been employed in the manufacture of novel flexible printed batteries [196]. 
Printing offers numerous advantages in scalability, labor requirement and greater productivity rates. Novel layering of electrode materials is also possible [105], [107].

Despite this, iron electrodes (used commercially in Nickel Iron Batteries solely manufactured by Sichuan Changhong Battery Co. Ltd.) are not produced using any printing methodology. Work in this chapter was performed with inks which are designed to be printed via screen printing. Electrochemical testing was used only to assess the mechanical stability of the electrodes produced, not to assess their performance.

\subsubsection{Low Temperature Route (Binders)}

In this chapter, the low temperature production route for iron electrode batteries is discussed. This involves heating at $200-400{ }^{\circ} \mathrm{C}$ with the addition of a binder to maintain structure.

Rapid processing routes are investigated to align with the desire to produce a system compatible with manufacture on a continuous roll to roll production line.

Low temperature routes are generally desirable as a reducing atmosphere is not required to prevent excessive oxidation. Additionally, there is a clear cost advantage in avoiding heating to the temperatures required for sintering. It is not expected that the long-term performance of a low-temperature electrode would match that of a high temperature sintered electrode - as it is anticipated that the mechanical stress upon charge/discharge would be damaging to a bonded electrode.

Various heating methods were considered, including conventional heating using an electrically heated furnace, which relies on convection and conduction. However, with the research aims including objectives to produce electrodes with a focus on speed of manufacture, high power heating systems are required. Microwave heating was considered, which shows promise for heating small powders rapidly [197]. However, this researcher had concerns about the uniformity of heating and that there would be localized hot and cold spots resulting in a poor-quality electrode. Ultimately, this researcher selected near infrared (NIR) furnace on the basis of availability, with the equipment having been used successfully by colleagues in the manufacture of photovoltaics [140]- 
[142]. As the heating is radiative it is considered it would be more even and an easier process to yield successful electrodes.

Solvent boil is an issue during rapid heating and furnace design combined with complex ink formulations may limit this. This will be explored in preliminary work which was also required to determine the appropriate substrate to use, the behavior of the iron ink during heating and the effect of heating to different temperatures is discussed in Section 3.2. Experimental work aimed at achieving the above stated desires to achieve rapid manufacture of electrodes continues in Section 3.3.

An alternative high temperature route (sintering) is discussed in Chapter 4.

\subsection{Preliminary Investigations: Low Temperature Route}

Here is discussed preliminary work central to the experimental research discussed in Section 3.3. This includes: immersion of iron and nickel substrates coated with ink; thermogravimetric analysis of the PTFE containing inks; and heating of the inks followed by scanning electron microscopy investigations. Details about the ink used is written in 3.3.1.

\subsubsection{Coated Iron and Nickel Substrates - Immersion Test}

This was intended to test the adhesion of the Gwent Electronic Materials Ltd. Inks when immersed in potassium hydroxide on a range of substrates.

Traditional manufacture of nickel-iron batteries (a common use of the iron electrode) utilized nickel plated steel for the substrate on to which the active material was applied [26]. No justification could be found of this use in literature and it was considered that this substrate may have been used purely for historical reasons and simplicity in manufacture of 'pocket plate' electrodes. Thus, an iron (or low carbon steel) substrate may offer advantages as a substrate for use on the iron electrode.

Traditionally, nickel plated steel was used as an electrode substrate. $0.5 \mathrm{~mm}$ annealed nickel sheet (Goodfellow metals) was used to simulate this. $0.5 \mathrm{~mm}$ sheets of iron (Goodfellow metals) was also used as an alternative substrate. A simple test was carried 
out by immersing the substrates coated with Gwent Electronic Materials 10\% PTFE iron ink (C2150413D4) in 5 M KOH (Sigma Aldrich) for 330 hours.

Substrate surface modification was performed. For the iron substrate, one sample was left as received, one sample was treated for 10 minutes with a plasma surface cleaner (Diener Electronics) to remove surface contaminants such as organic residue and modify the surface oxides and the final sample was abraded with p40 sandpaper (3M) until visible scarring was present on the entire surface. These actions were repeated with the nickel substrate.

Prior to tape casting, samples were washed with acetone and dried. Samples were tape cast using a tape thickness of $0.13 \mathrm{~mm}$ onto $5 \times 5 \mathrm{~cm}^{2}$ coupons of all substrates. Curing was performed on a hot plate; samples were heated to $180^{\circ} \mathrm{C}$ for 15 minutes.

The results of this testing are visible in Figure 27. Complete debonding was observed in the 'plasma treated' samples on both substrates. It is thought that the plasma surface treatment negatively affects the ability for the ink to bond to the substrate. Partial debonding was observed in the 'as received' samples on both substrates. This performance is better than the 'plasma treated' samples, although is not satisfactory. Debonding was not observed in the samples that had been mechanically abraded. Discoloration was observed in the sample where a nickel substrate was used. This is thought to be due to bimetallic corrosion between the iron in the ink and nickel substrate. This was not observed in the sample that used the iron ink on the iron substrate.

It is worth noting that the bimetallic corrosion which appeared to occur between the nickel substrate and iron coating may not occur in the same manner in an assembled cell due to the potential difference between the negative and positive electrodes.

Based on the findings reported here, a low carbon steel was chosen for further testing in Chapter 3. Properties would be similar to the iron substrate tested above as it was available directly from the project sponsor. This preliminary work showed that adhesion of the ink to the substrate would be a significant challenge in the research discussed in this thesis. 


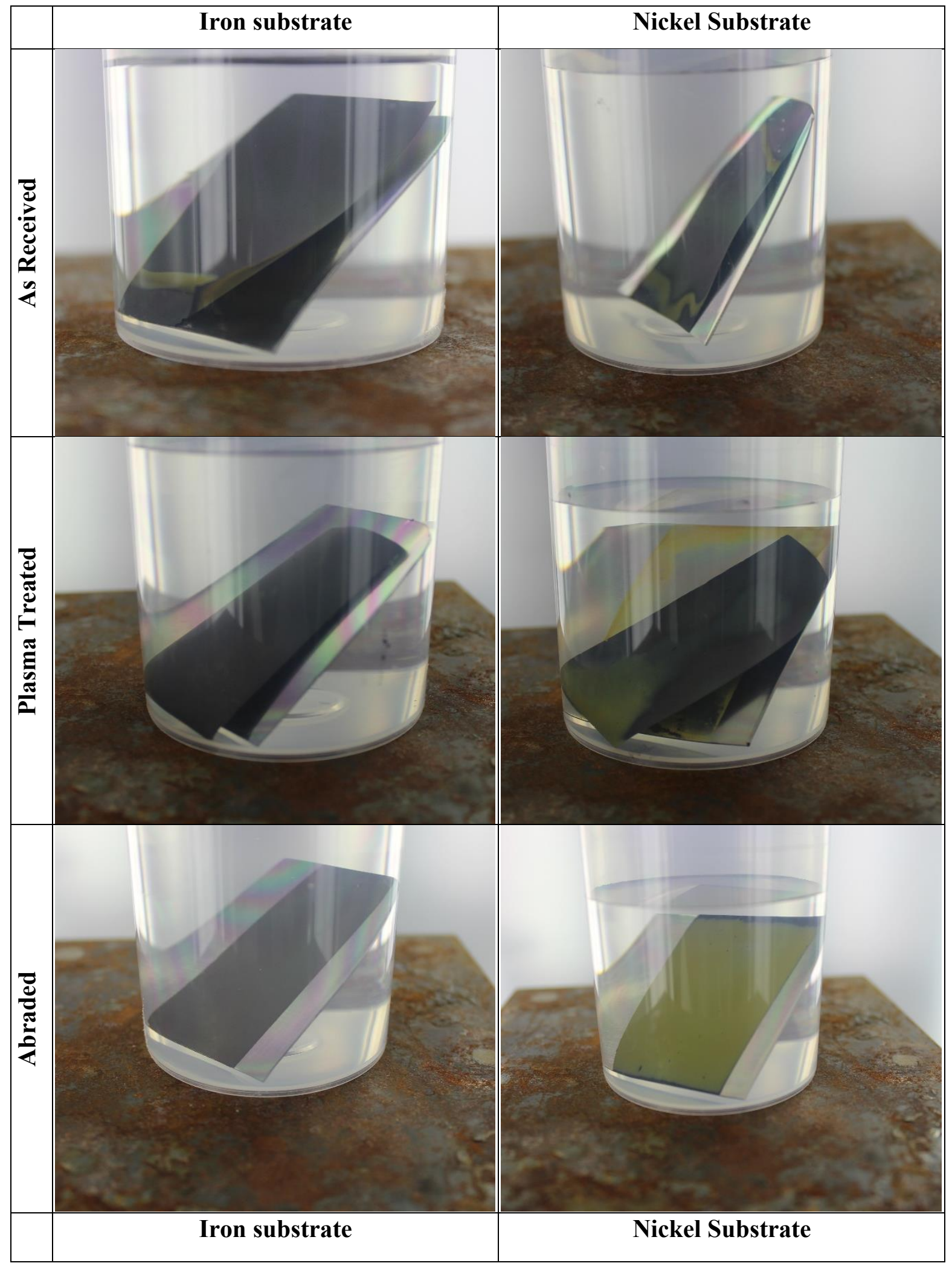

Figure 27 Results of 330 hour immersion test of coated iron and nickel substrates with various surface treatments 


\subsubsection{Thermogravimetric analysis of iron ink}

In order to investigate the temperatures which solvent is removed from the ink, a Thermogravimetric Analysis (TGA) was performed as per 2.6.1. The samples were heated from $50{ }^{\circ} \mathrm{C}$ to $550{ }^{\circ} \mathrm{C}$ at $20^{\circ} \mathrm{C} / \mathrm{min}$. This test was performed in air. Additionally, Derivative Thermogravimetry (DTG) was calculated from the TGA data and both are presented in Figure 28.

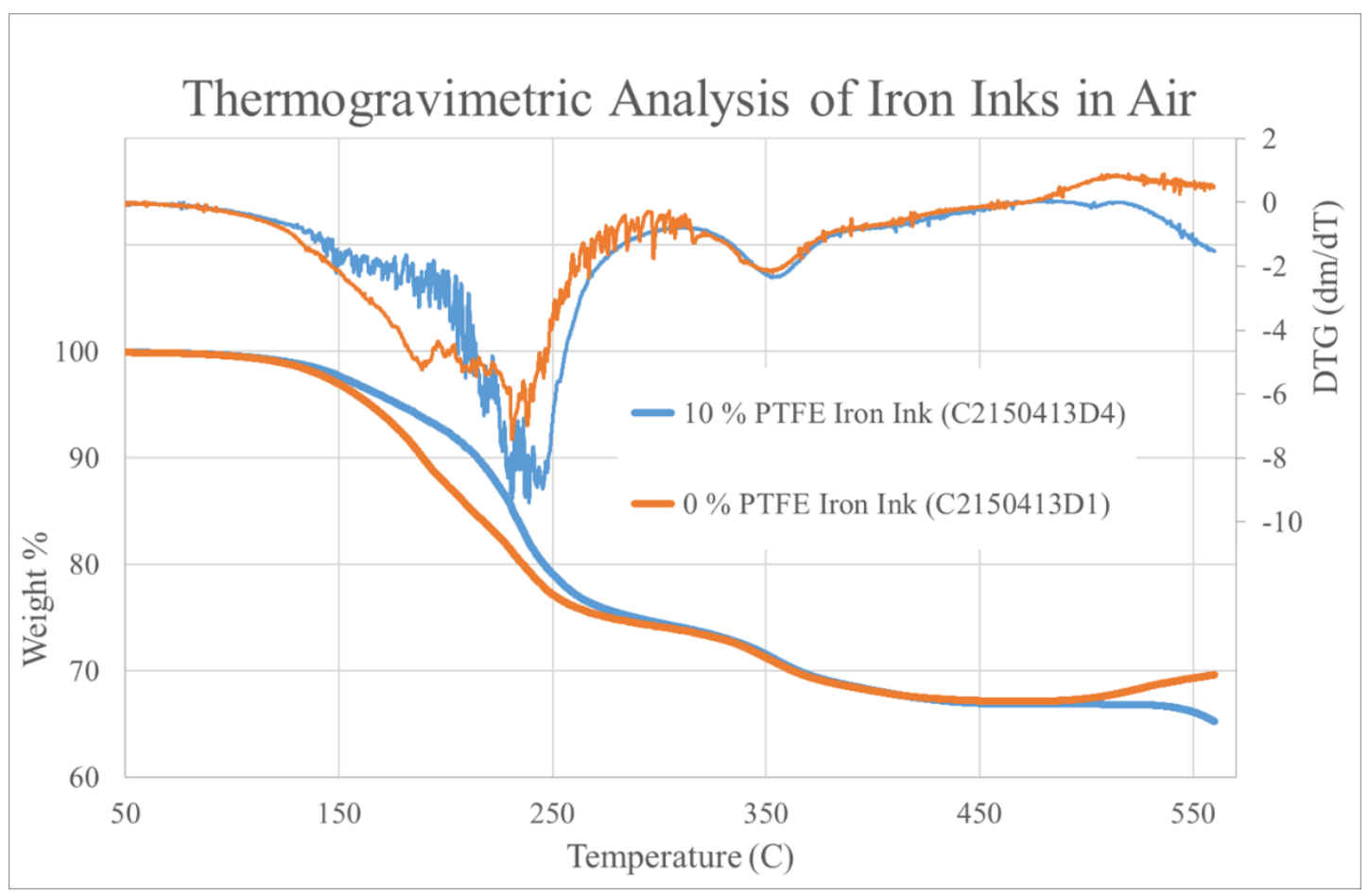

Figure 28 TGA of Gwent Electronic Materials Ltd. iron inks in air. Secondary axis is derivative thermogravimetry (DTG).

A subtle yet significant contrast is visible between the ink which contains PTFE and the ink which does not contain PTFE, for both TGA and DTG.

Below $150^{\circ} \mathrm{C}$, there is agreement between the two curves and a small mass loss, this is likely attributable to a small amount of water or solvent vaporization. The curves then diverge between $140{ }^{\circ} \mathrm{C}$ and $300{ }^{\circ} \mathrm{C}$. During this temperature range, it is expected that both solvent and water will be vaporizing - the PTFE in the PTFE-containing ink was stabilized in water. Accordingly, this author anticipates that this ink will have a higher water content than the non PTFE-containing ink. Whilst both inks reach the same mass 
at $300{ }^{\circ} \mathrm{C}$, the rate of mass loss in the PTFE-containing ink is initially slower. This is thought to be due to the high thermal capacity of water resulting in a 'lag' in vaporization, or possibly, a chemical reaction between the water and other species in the PTFE-containing ink. An Isothermal TGA or a slower TGA heating rate may provide greater insight to this contrast between the two inks. It is not expected for the chemical reaction of thermal decomposition of PTFE to occur in this temperature range, hence this author attributing the effect to the water content in the PTFE-containing ink.

Between $300^{\circ} \mathrm{C}$ and $400^{\circ} \mathrm{C}$ there is a slight hump in the plot, initially mass loss appears to slow, where it assumed there are no volatile components being removed, then $\left(350^{\circ} \mathrm{C}\right)$ a less volatile solvent undergoes a phase transition to a gaseous state, resulting in the mass loss in the sample. Use of a TGA combined with gas chromatography or mass spectrometry may allow a researcher to better identify the solvent content and mass loss reactions.

At $500{ }^{\circ} \mathrm{C}$, the curves diverge again. This appears to be as a result of the thermal degradation of PTFE, in agreement with literature [198]. It is important to note that as the test was completed in air, oxidation of iron will also be occurring at this temperature. This physical uptake of oxygen into the iron is most likely what gives rise to the increase in mass of the non-PTFE sample. This mass uptake will be masked in the PTFE containing sample, due to it coinciding with the thermal degradation of the PTFE in the ink. Performing the TGA in an alternative atmosphere such as nitrogen may help to investigate this further, however the chemical degradation of PTFE would also be altered.

The DTG data shows significant noise in the $150-300{ }^{\circ} \mathrm{C}$ region, despite the smoothing used (2.6.1). Whilst an element of this will be due to random fluctuation, it is likely that some could be attributed to the physical effects of decomposition, where volatile compounds leave the sample. The fact that the noise is particularly present in this region supports the above analysis that this is the main region for removal of water and solvents. Also, secondary reactions which do not directly contribute to the mass loss may be a factor in the noise observed. More noise is seen on the sample containing PTFE, which could be due to minor differences in the sample size but, is considered more likely to be due to the different compositions of the sample and the presence of water in the ink. 
The TGA suggests that the PTFE is significantly more thermally stable than suggested by the manufacturer of the ink, although it is acknowledged that degradation of PTFE is unlikely to be a binary process. If a low temperature route is used on the PTFE-containing ink, then efforts should be made to keep the oven temperature below $500{ }^{\circ} \mathrm{C}$. However, fine control of heating temperature is not possible with all heating techniques.

Further information relating to the $10 \%$ PTFE ink and its response to heating can be found below.

\subsubsection{Hot plate investigation for PTFE inks}

In addition to the TGA performed previously, practical tests were performed using a hot plate at different temperatures to investigate the effect of temperature on the PTFE containing ink.

These tests were included as they show the thermal stability of the PTFE binder which is used in the ink.

Samples were prepared by again coating $5 \times 5 \mathrm{~cm}$ iron coupons $(0.5 \mathrm{~mm}$ thickness $)$ with a $0.13 \mathrm{~mm}$ coating of $10 \%$ PTFE Iron containing Ink (Gwent Electronic Materials Ltd. C2150413D4).

Samples were then heated on a lab hot plate for 5 minutes at various temperatures. Investigation of the center of the coated area was completed using Scanning Electron Microscopy (Hitachi TM3000). 


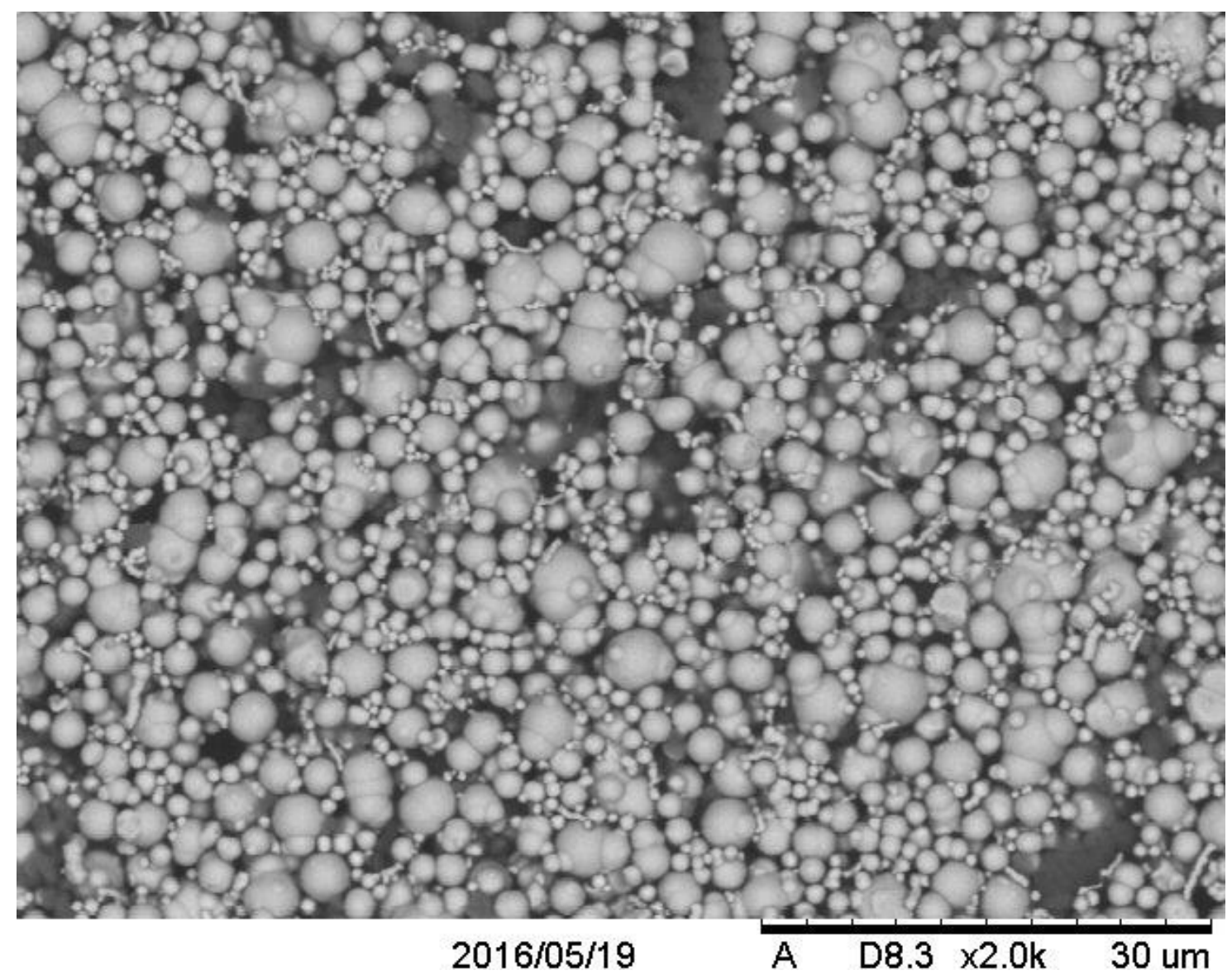

Figure 29 Preliminary test: $\mathrm{C} 2150413 \mathrm{D} 4$ heated to $260{ }^{\circ} \mathrm{C}$ for 5 minutes

Figure 29 shows evidence of PTFE (dark grey), this was assessed further using Energydispersive X-ray spectroscopy (EDS) analysis.

EDS was performed in order to aid identification of key phases which are frequently observed via scanning electron microscopy. An example of this is seen in Figure 30. PTFE is observed through the identification of fluorine and carbon. This also allows for identification of oxidation, which is not observed in significant quantities here. When this image was compared to its SEM counterpart (e.g. Figure 34 and Figure 35) it was possible to determine that the darker grey regions commonly observed are PTFE binder which has not substantially thermally degraded. 


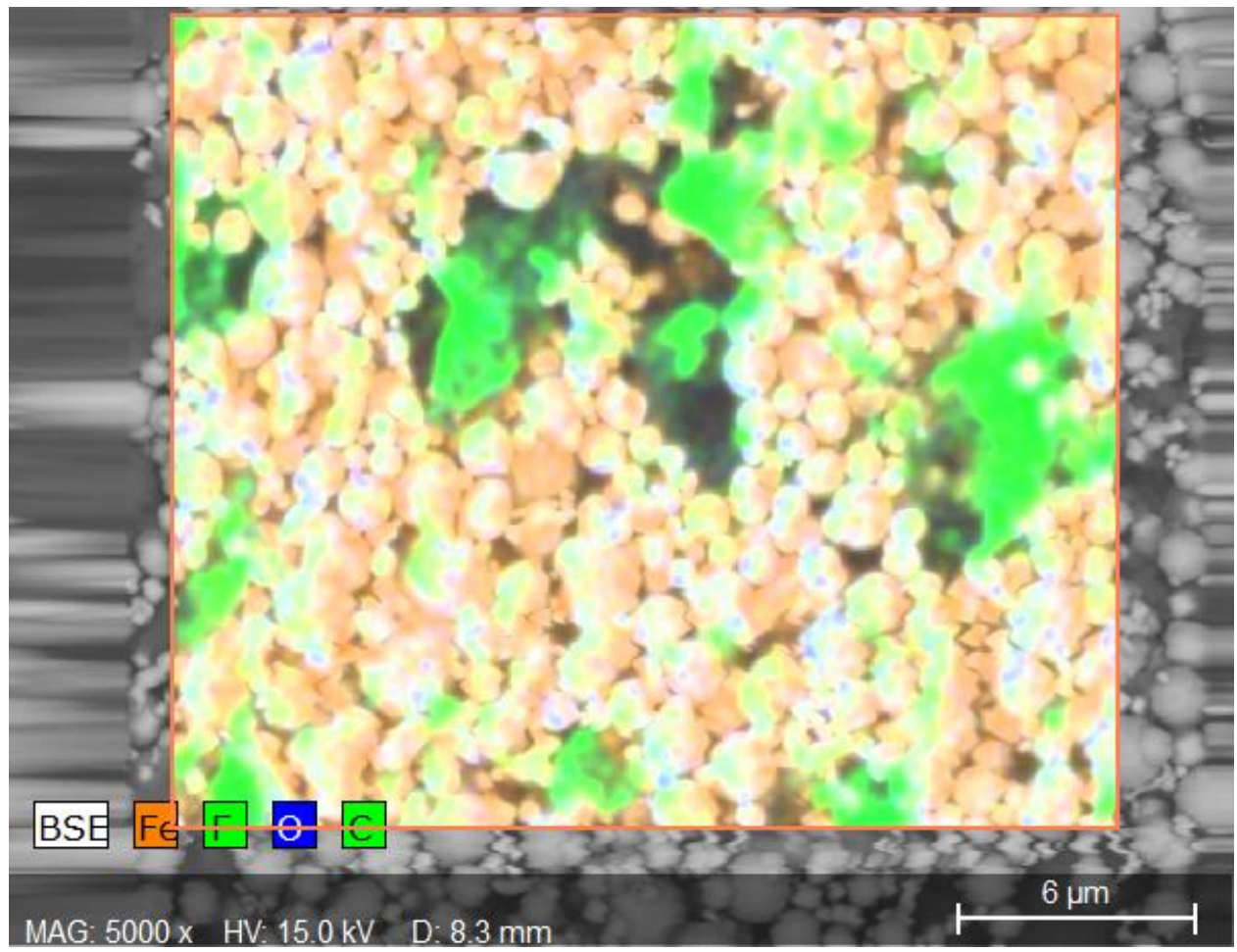

Figure 30 EDS performed on iron containing ink in order to identify key phases

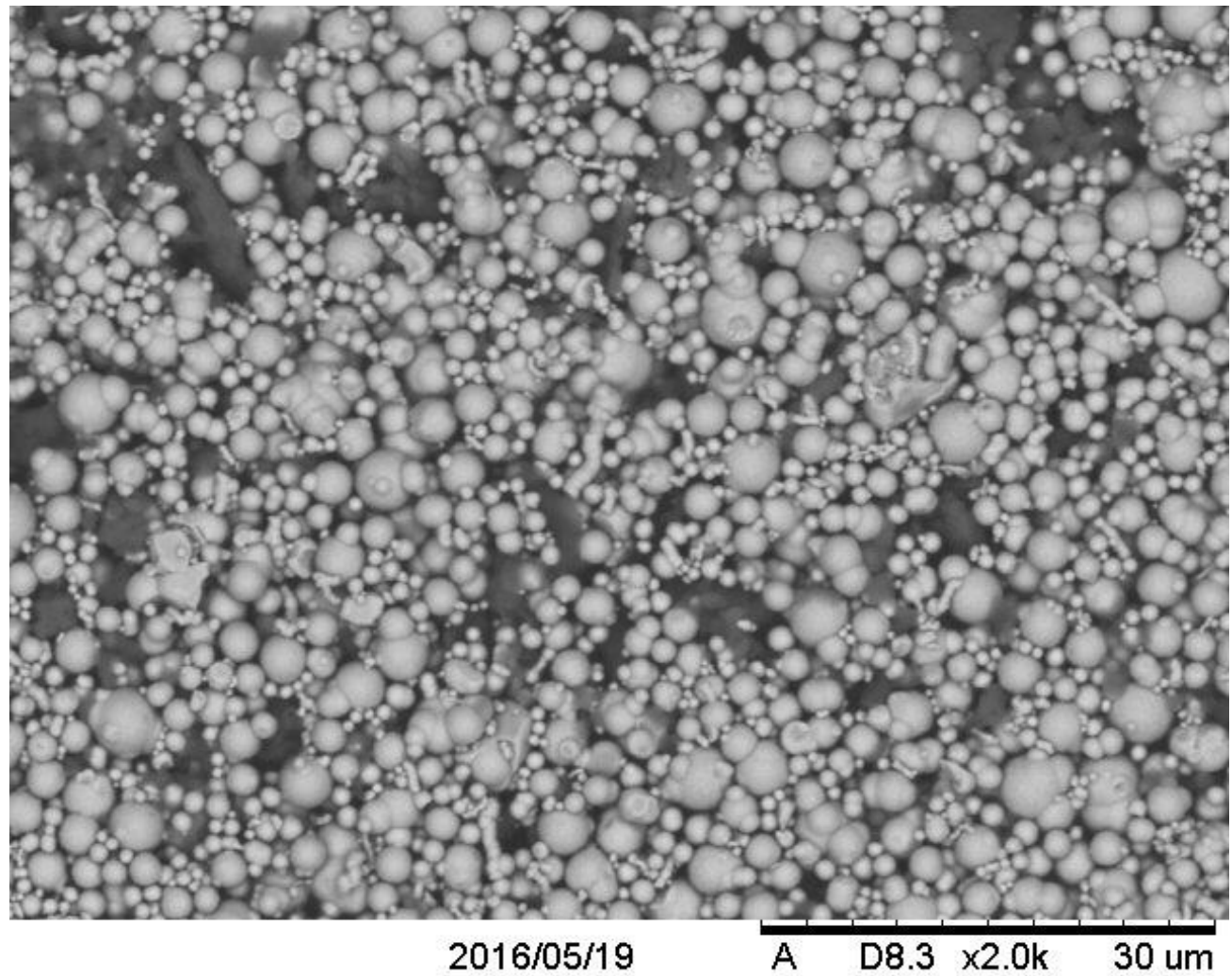

Figure 31 Preliminary test: C2150413D4 Heated to $280^{\circ} \mathrm{C}$ for 5 Minutes 
There is no significant difference to the resulting surface of the ink on a sample which has been heated to $280{ }^{\circ} \mathrm{C}$ (Figure 31 ).

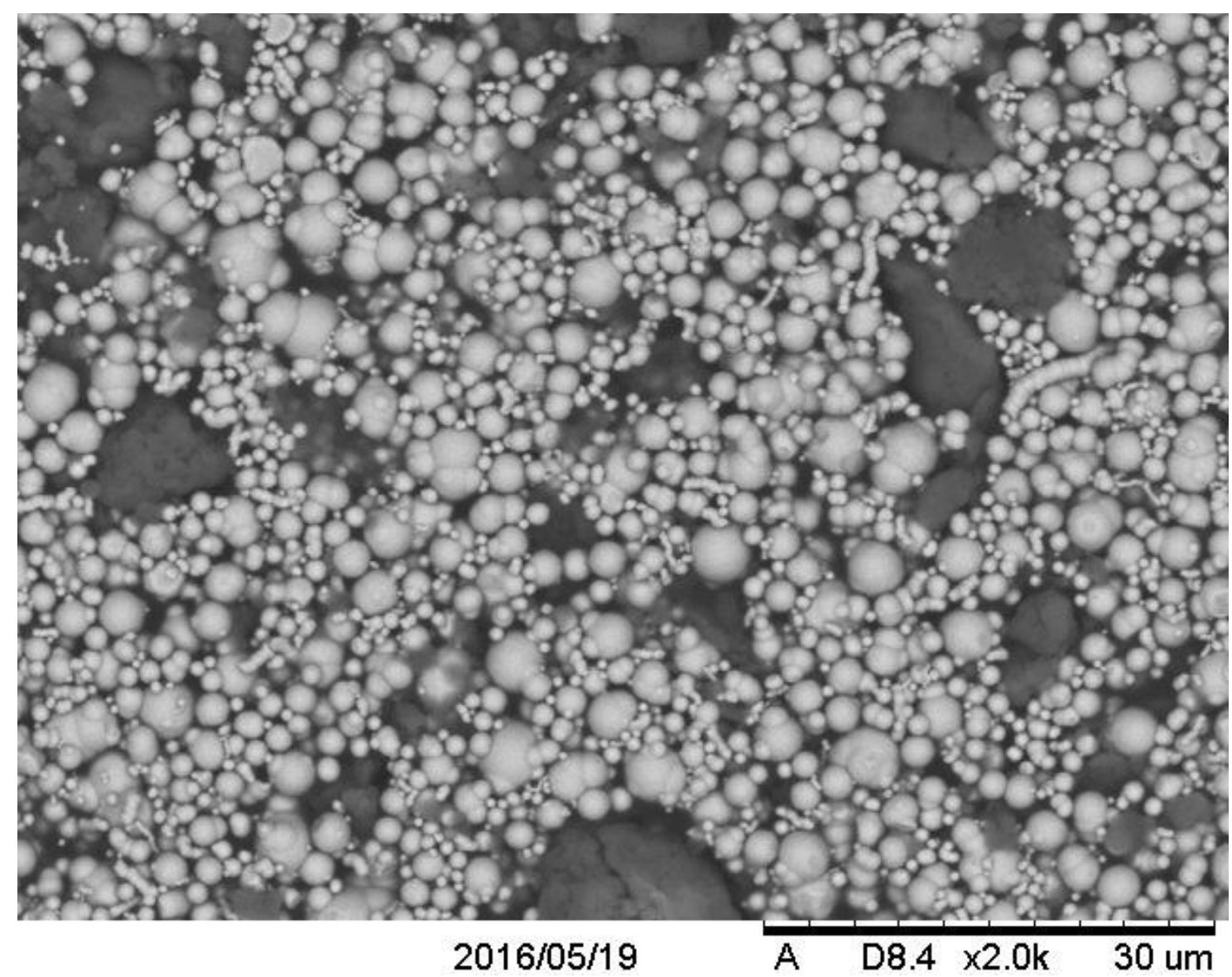

Figure $32 \mathrm{C2150413D4}$ Heated to $300{ }^{\circ} \mathrm{C}$ for 5 Minutes

PTFE decomposition appears to have begun in the sample which was heated to $300{ }^{\circ} \mathrm{C}$, this can be seen in Figure 32 where the PTFE appears to have coalesced into larger deposits. 


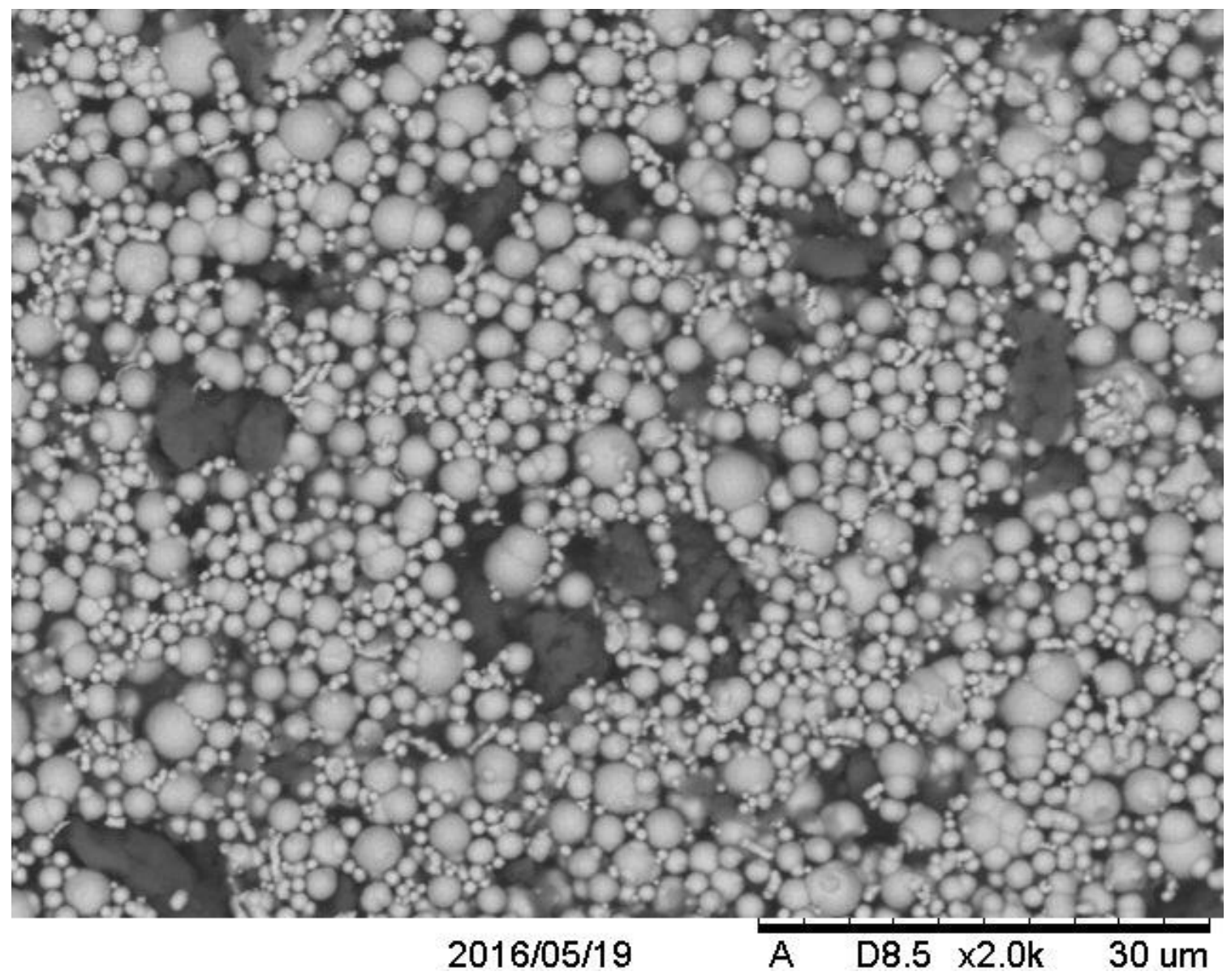

Figure 33 C2150413D4 Heated to $320{ }^{\circ} \mathrm{C}$ for 5 Minutes

At $320^{\circ} \mathrm{C}$ (Figure 33) further minor decomposition of PTFE is visible. Large amounts of PTFE are still visible, so it is unlikely that significant decomposition has occurred. 


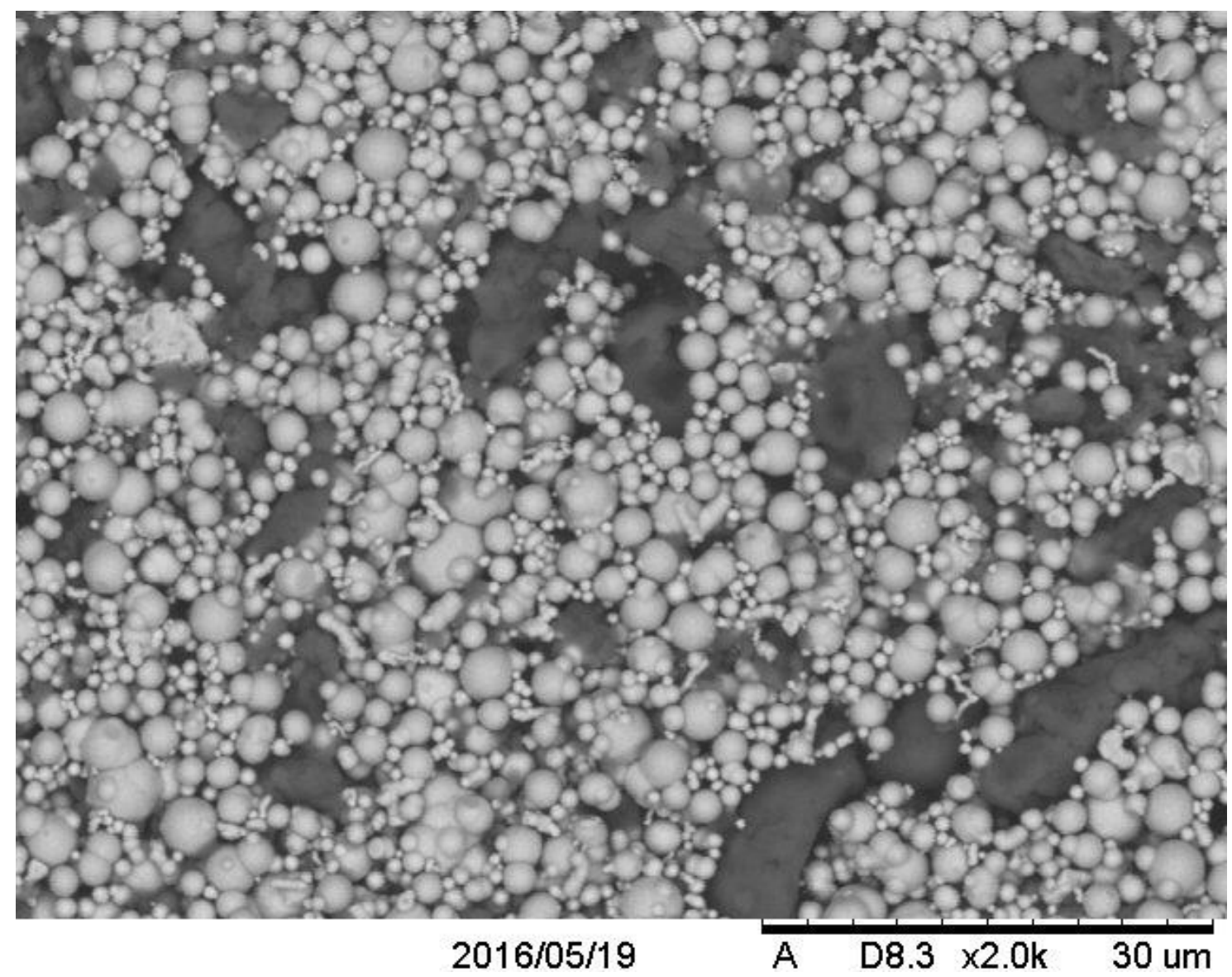

Figure 34 SEM of C2150413D4 Heated to $340{ }^{\circ} \mathrm{C}$ for 5 Minutes (initial hot plate temperature $320^{\circ} \mathrm{C}$ )

Samples heated above $320^{\circ} \mathrm{C}$ required a more gradual heating process to avoid boiling of the solvent in the ink. They were placed on the hot plate at $320{ }^{\circ} \mathrm{C}$ and the hot plate was adjusted in order to heat to the sample to $340{ }^{\circ} \mathrm{C}$. As with previous tests, the 5 minute heating time was used. Placing a sample directly on the hotplate at $340{ }^{\circ} \mathrm{C}$ resulted in solvent boiling of the ink so was avoided.

Further decomposition of the PTFE binder in the ink was observed in Figure 34, however, PTFE is still clearly present in this sample. 


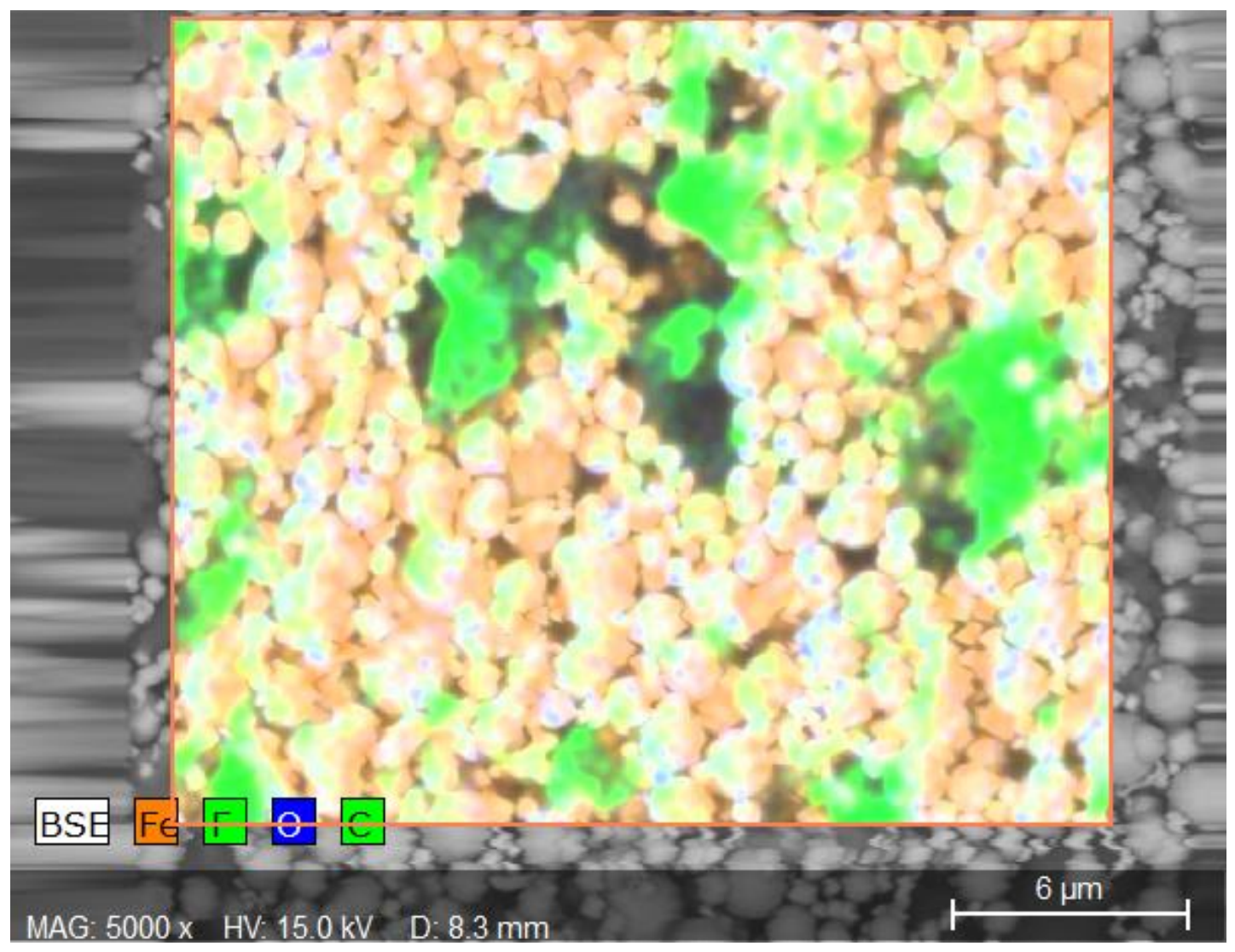

Figure 35 EDS of C2150413D4 heated to $340{ }^{\circ} \mathrm{C}$ for 5 Minutes (initial hot plate temperature $320^{\circ} \mathrm{C}$ )

EDS examination of is shown in Figure 35. PTFE is shown in green through the illumination of fluorine and carbon. Only minor oxidation is observed in some areas, although this is to be expected when iron is heated in air at $340{ }^{\circ} \mathrm{C}$.

It is clear that PTFE is present well above $300{ }^{\circ} \mathrm{C}$, although it is possible that its effectiveness as a binder is reduced. PTFE, as present in the Gwent Electronic Materials ink, appears to have a higher than expected thermal stability. This suggests that at the larger heating rates which are experienced during NIR heating, PTFE should not be excessively decomposed, as is desirable. 


\subsection{Materials}

\subsubsection{Ink Development}

Iron containing inks were provided by Gwent Electronic Materials to the specification of this researcher. It was identified that ink should be capable of being screen printed to enable future capability, thus viscosity and other relevant properties were specified accordingly.

The source of active material in all inks was Carbonyl Iron $\geq 97 \%$ Fe (Sigma Aldrich). This was specified based upon the particle size (typically 1-10 $\mu \mathrm{m}$ ) and its appropriateness for the application. The researcher considered that a larger particle size for the ink would reduce the available surface to support electrochemical reactions, resulting in poorer performance when compared with a smaller particle size. Further, a particle size below $1 \mu \mathrm{m}$ may reduce the ability of the electrolyte to wet the active material. The merits of carbonyl derived iron were discussed in 1.6.2 and 1.6.4. Carbonyl iron is spherical, thus morphology was not an variable available to this researchr.

PTFE and PE were both considered as a suitable binder, although PTFE was chosen under advice of the ink manufacturer and it has also been used successfully by Periasamy et al [77]. Water-stabilized Ultraflon MP 10 PTFE (Lawrence Industries) was recommended by the ink manufacturer as an appropriate product.

The resulting ink contained 10\% PTFE and a balance of Iron and is catalogued by Gwent Electronic Materials as C2150413D4, with more details in Appendix A.

Following specification of the active material and binder system, Gwent Electronic Materials specified a suitable resin/solvent composition. The manufacturer was unable to disclose the exact chemical composition of the ink, they did state that the resin/solvent (vehicle) system should be volatized by $250{ }^{\circ} \mathrm{C}$. Above this temperature, iron and PTFE would remain. The PTFE would begin to decompose at $300{ }^{\circ} \mathrm{C}$. From this information, the PTFE ink should be heated to around $300^{\circ} \mathrm{C}$. This relationship was explored in section 3.2.3. 


\subsubsection{Substrates}

Substrates of low carbon steel were used. They had the following surface finishes: As received, wire brushed or ground. They were produced as described in 2.1.1. A low carbon steel is selected a substrate material as it is available from the project sponsor and is of a similar composition and electrode potential to the iron discussed in the preliminary work in 3.2.1. It is not considered to be the active material in the resulting electrode.

\subsection{Rapid Low Temperature Electrode Manufacture Route}

Herein, proposed methods of manufacture of an iron electrode using a Near Infrared (NIR) oven are investigated following the preliminary work discussed in section 3.2.

Low cost manufacture of electrodes can be achieved by combining the electrode material with a binder [22]. One factor in this reduced cost is that an inert atmosphere is not required.

NIR heating was investigated as the method is relatively inexpensive and has a high throughput making it suitable for implementation on to a production line, in contrary to traditional heating methods such as hot plates. The technique has been used with success in other research areas [140]-[142]. Whilst heating is rapid, there are several disadvantages:

- Heating may not be uniform over the surface of the sample

- The binder incorporated into the ink will remain after heating - this results in a lower weight/volume fraction of active material in the coating

- Long term performance and stability of plastic-bonded electrodes is unknown; however, it is unlikely to compete with traditional pocket plate electrodes or sintered electrodes

\subsubsection{Experimental Method}

Due to the researchers' concerns about the adhesion of the ink to the substrate and in line with the preliminary work in this chapter (3.2.1), a low-carbon steel with various surface 
treatments (2.1.1) was used as a substrate during this stage of the investigation. A coated area of $20 \mathrm{~mm} \times 50 \mathrm{~mm}$ was used on a substrate sized $70 \mathrm{~mm} \times 70 \mathrm{~mm}$.

Samples were coated with 10\% PTFE Iron containing screen-printing ink (Gwent Electronic Materials Ltd. C2150413D4). A glass rod was used to tape cast a $20 \mathrm{~mm}$ x 50 $\mathrm{mm}$ layer of thickness $0.13 \mathrm{~mm}$ centrally on the substrate as per 2.1.3.

\subsubsection{NIR Heating}

The furnace described in 2.1.4 was used to heat the samples, both to remove solvent and cure the ink.

Initial testing, recorded in Table 2, was completed using coupons of untreated low carbon steel, in order to gauge the amount of power and line speed which was appropriate for the sample. Coupons sized 30 x $40 \mathrm{~mm}$ were used during this stage of testing. It was apparent that there was a fine balance between failing to remove the volatile organic compounds in the ink and overheating the ink resulting in solvent boil. Solvent boil was considered undesirable due to the difficulty in reproducibility and the non-uniform coating produced due to convection effects within the ink during solidification.

It was also observed that the device would heat up cumulatively during operation, which meant that if two identical samples were run immediately after each other, then the second sample would receive significantly more radiated heating from the areas surrounding the NIR lamps. This would result in the second coating being more prone to solvent boil. For this reason, during testing, the Adphos NIR was allowed to cool between samples. Cooling was considered complete when the thermostatically-controlled cooling fan switched off. This ensured that heating was consistent from sample to sample. Line speeds were gradually reduced during preliminary testing to ensure complete VOC removal and even heating of the samples. In some cases, the sample was passed through the NIR multiple times. 
Table 2 Preliminary NIR Heating of 10\% PTFE Gwent Electronic Materials Fe

Ink on $0.3 \mathrm{~mm}$ low carbon steel substrate

\begin{tabular}{|l|l|}
\hline Adphos NIR Settings & Result \\
\hline $2.0 \mathrm{~m} \mathrm{~min}^{-1}$ and $10 \%$ & Insufficient heating- sample still wet \\
\hline $2.0 \mathrm{~m} \mathrm{~min}^{-1}$ and $20 \%$ & Insufficient heating- sample still wet \\
\hline $2.0 \mathrm{~m} \mathrm{~min}^{-1}$ and $30 \%$ & Severe solvent boil \\
\hline $2.0 \mathrm{~m} \mathrm{~min}^{-1}$ and $25 \%$ & $\begin{array}{l}\text { Minor solvent boil however sample not } \\
\text { completely dry - line speed reduced }\end{array}$ \\
\hline$\left(1.0 \mathrm{~m} \mathrm{~min}^{-1}\right.$ and $\left.20 \%\right) \times 2$ & Insufficient heating- sample still wet \\
\hline$\left(1.0 \mathrm{~m} \mathrm{~min}^{-1}\right.$ and $\left.20 \%\right) \times 4$ & $\begin{array}{l}\text { Minor solvent boil however sample not } \\
\text { completely dry - line speed reduced }\end{array}$ \\
\hline$\left(0.5 \mathrm{~m} \mathrm{~min}^{-1}\right.$ and $\left.15 \%\right) \times 6$ & Insufficient heating- sample still wet \\
\hline$\left(0.5 \mathrm{~m} \mathrm{~min}^{-1}\right.$ and $\left.18 \%\right) \times 4$ & Good overall, some VOC retained in a few areas \\
\hline $\begin{array}{l}\left(0.5 \mathrm{~m} \mathrm{~min}^{-1} \text { and } 20 \%\right) \times 2 \\
\left.\mathrm{~m} \mathrm{~min} \mathrm{~min}^{-1} \text { and } 20 \%\right)\end{array}$ & Solvent Boil \\
\hline $\begin{array}{l}\left(0.5 \mathrm{~m} \mathrm{~min}^{-1} \text { and } 16 \%\right) \text { and then } \\
\left(0.5 \mathrm{~m} \mathrm{~min}^{-1} \text { and } 20 \%\right)\end{array}$ & $\begin{array}{l}\text { Good - no solvent boil and VOC appears to be } \\
\text { removed. }\end{array}$ \\
\hline
\end{tabular}

The optimum heating regime (denoted by '*') was tested on a sample sized $30 \times 40$ $\mathrm{mm}$. It was found that when a larger sample was used, increased heating occurred due to the greater surface area for NIR absorption. Due to this, the larger low temperature samples were heated as follows: $0.5 \mathrm{~m} \mathrm{~min}^{-1}$ and $14 \%$ (repeated once) and then $0.5 \mathrm{~m}$ $\min ^{-1}$ and $16 \%$. This operation took two minutes to complete - however, the samples were not being irradiated all of this time.

The final operating parameters used were $0.5 \mathrm{~m} \mathrm{~min}^{-1}$ and $14 \%$ (repeated once) and then $0.5 \mathrm{~m} \mathrm{~min}^{-1}$ and $16 \%$, on samples with a coated area of $20 \mathrm{~mm}$ x $50 \mathrm{~mm}$ on a substrate sized $70 \mathrm{~mm} \times 70 \mathrm{~mm}$. An example structure of an electrode produced in this manner is shown in Figure 36. For electrochemical analysis, one sample was produced for each of the substrate surface treatment conditions: as received, ground and wire brushed. 


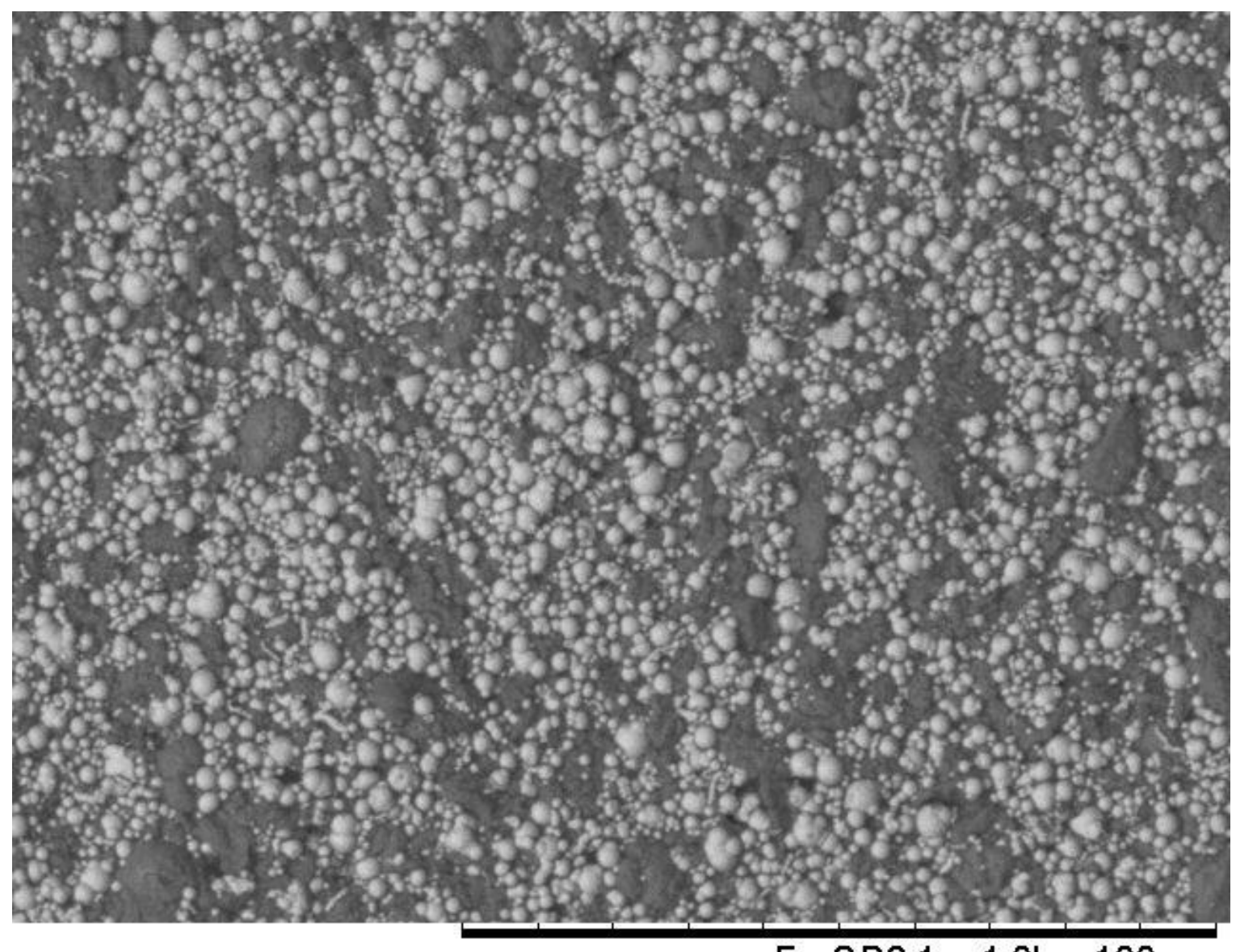

F $\quad$ SD8.1 x1.0k $100 \mathrm{um}$

Figure 36 PTFE containing Iron ink sample heated on AdPhos NIR

Heating was completed in approximately 90 seconds, following this the three coated substrates were each cut into 5 samples using a guillotine. This resulted in a substrate with a coated area of $10 \mathrm{~mm} \times 20 \mathrm{~mm}$.

\subsubsection{Electrochemical cycling}

Samples were tested using a 64-channel Arbin SCTS battery cycler under galvanic conditions at room temperature to assess if the electrodes produced could function as a viable electrode in a cell. A three-electrode setup was used. The working electrode was produced as above. Cells were cycled at a $0.2 \mathrm{C}$ rate from 0.6 to $1.4 \mathrm{~V}$ vs. $\mathrm{Hg} / \mathrm{HgO}$ as described in 2.1.5.

Despite use of a range of heating conditions in the near infrared furnace, it was not possible to produce electrodes which withstood cycling as a half cell. All electrodes 
manufactured debonded from the substrate upon the first attempt to discharge. By the third cycle no active material remained on the substrate and cycling was stopped. This researcher was unable to plot data in a meaningful way as, due to the failure of the electrodes, no capacity was recorded for discharge of the electrode.

The surface modification of the substrate had no discernible effect on the performance of the electrodes given the failure methods were all the same. It is thought that the adhesion of the active material to the substrate was insufficient to survive the volume expansion during charge/discharge, resulting in the coating shearing off the substrate.

\subsection{Conclusions}

In this chapter, various methods for the rapid manufacture of printed iron electrodes using NIR heating and binders have been discussed. This work was attempted as an alternative to traditional 'pocket plate' designs commonly found in commercially available iron electrode batteries [26]. Printed electrodes are desirable as they have a potential to be produced at a lower cost and at a greater rate than in traditional manufacture.

Electrodes were produced using a low temperature production route which utilized a PTFE binder to assist in forming a stable electrode. Heating was completed in less than 90 seconds. Steel substrates with various pre-treatments were used.

All electrodes produced by the rapid low temperature NIR route suffered total failure due to the delamination of the coated material. The type of substrate pre-treatment/preparation (2.1.1) appeared to have no effect on the stability of the electrode in this small dataset. Additionally, as the preliminary work (3.2) did not involve electrochemical cycling of the electrodes, it is not known if the same delamination effect would have occurred in those under cycling. It is possible that this cause of delamination could have been related to the use of NIR to heat the ink, or equally due to a fundamental lack of adhesion of the ink to the substrate under cycling conditions.

It should also be noted that the Fe ink used was not formulated for rapid heating. An alternative ink formulation may have resulted in less susceptibility to solvent boil however this might have come at the expense of ease of printing and mean that the ink would not be suitable for screen-printing. 
It is deemed that manufacture of a low temperature electrode using rapid heating and a binder requires further investigation. Due to this failure, further experimentation using binders and a low temperature curing route were not continued.

Sintered electrodes, produced at higher temperatures, are discussed in Chapter 4.

\subsection{Further Work}

Further work in this area should focus on the adhesion between the ink and the substrate, as this was the biggest failing in this research. Metallic bonding between the coating and the substrate are generally not possible at temperatures below $0.5 \mathrm{Tm}$, however use of a flux may aid in forming metallic bonds.

If electrodes which cycle successfully can be produced, there are a range of other publications that could be used to compare results [20], [22], [81]-[83].

A layered iron electrode could also be of interest, following on for work described by Huang et al [105]. 


\section{Chapter 4: Printed Sintered Iron Electrodes for Low Cost Alkaline Batteries - High Temperature Route}

\subsection{Preface}

Work in this chapter is a direct continuation of Chapter 3, however here the author is focusing on high temperate electrode production, involving sintering of the active material in the electrode. The general points regarding the advantages of printed electrodes over traditional manufacture which were made in 3.1 are valid for Chapter 4 also.

\subsection{Introduction}

In this chapter, the high temperature production route for iron electrode batteries is discussed. Temperatures above $650{ }^{\circ} \mathrm{C}$ are required in order to sinter the iron in to one metallically bonded and physically connected mass. The speed of this bonding can be increased by increasing the sintering temperature [135].

Sintered electrodes have advantages including improved adhesion to the substrate and improved cohesion of the iron particles, due to the metallic bonding between previouslydiscrete particles of iron. It may be possible to produce an electrode with a longer cycle life, due to the inherent increased mechanical stability in a metallically bonded electrode [19].

Uniform heating will produce highly repeatable samples and, combined with advanced high-power heating technologies, rapid manufacture should still be possible. Novel rapid heating methods of manufacture of iron electrodes align with the desire to produce a system compatible with manufacture on a roll to roll production line. Various rapid heating methods such as microwaving, induction heating and near infrared heating were considered, however, these were not available to the researcher with sufficient power to reach the required temperatures. Ultimately, the Hot Dip Simulator (HDS), described in 2.1.4 was used as it was the only equipment available to the researcher with the required heating power to quickly sinter the iron electrodes. 
There is an inherent cost increase in the use of this high temperature processing route, partially because of the increased thermal energy required and partially as a reducing atmosphere is essential to avoid excessive oxidation.

Development work necessary for successful sintering is discussed here, including use of substrates with various surface roughening treatments. As previously, iron ink used is suitable for screen printing and electrochemical testing was used only to assess the mechanical stability of the electrodes.

\subsection{Materials}

\subsubsection{Ink Development}

Ink used in this chapter is as specified by the researcher and manufactured by Gwent Electronic Materials, in accordance with the previous description in 3.3.1. A variant without the PTFE binder was produced specifically for the sintering work, with a higher volume of the active material (carbonyl iron). The polymeric binder was not required as it would not survive the sintering temperatures, whilst also adding cost to the ink. This is recorded by the manufacturer as C2150413D1, with more details in Appendix A.

\subsubsection{Substrate}

As per Chapter 3, low carbon steel substrate was prepared in a range of surface finishes: as received, wire brushed or ground, as per 2.1.1. In initial testing (4.5.1), all substrate was in the 'as received' form to reduce expenditure. Again, a low carbon steel is used due to the availability from the project sponsor and to simulate the potential production route.

\subsection{Method}

Substrate with each of the 2.1.1 surface modifications was cut to size $(200 \times 85 \mathrm{~mm}$ with a $35 \mathrm{~mm}$ chamfer on each corner). Coatings of iron-containing screen printing ink (Gwent Electronic Materials Ltd. C2150413D1) sized $20 \mathrm{~mm}$ x $80 \mathrm{~mm}$ and 0.13 or $0.26 \mathrm{~mm}$ were applied on to each substrate type via tape casting as per 2.1.3. 
Sintering was performed using a hot dip simulator and, in some cases, a lab hot plate was used to dry the ink prior to sintering (2.1.4). Analysis of sintering was performed using a Hitachi TM3000 scanning electron microscope (SEM) (2.6.2). This process was iterative in order to find optimum values and is detailed in 4.5.

Electrochemical cycling was performed using a 64 channel Arbin SCTS battery cycler under galvanostatic conditions at room temperature, as described in 2.1.5.

\subsection{Experimental}

Several experimental conditions were tested prior to making samples suitable for electrochemical testing. In this section, the process involved in finding suitable variables to use to manufacture electrodes is discussed. Due to uncertainty about which method would be successful, it was not possible to proactively design a matrix of experimental conditions to attempt. As a result, this researcher attempted electrode manufacture and then modified the experimental conditions iteratively on the basis of the electrode that was produced.

\subsubsection{Initial Sintering Conditions}

It was not initially known whether the infrared furnace in the hot dip simulator would have the capability to sinter iron. As preliminary work, an initial attempt to sinter iron was made in order to establish if it was within the operating parameters of the equipment available to the researcher.

Iron ink was coated on to the substrate at a $45^{\circ}$ angle (Figure 38) with a wet film thickness of $0.13 \mathrm{~mm}$. The coating was orientated in this manner to examine the uniformity of the heating using the infrared HDS furnace. It was not heated using a hot plate prior to heating in the hot dip simulator.

\section{Hot Dip Simulator (HDS) temperature profile}

The HDS (2.1.4) was programmed to heat to $150{ }^{\circ} \mathrm{C}$ for 5 minutes, then to $300{ }^{\circ} \mathrm{C}$ for a further 5 minutes - this was intended as a solvent burn off phase to avoid any solvent boil, as discussed in 3.4.1. This was followed by a sintering phase at $1000{ }^{\circ} \mathrm{C}$ for 5 minutes 
- chosen at 0.65 of the melting point of iron. A typical heating cycle is shown in Figure 37 including the HDS set temperature, where a slight $(0.5 \mathrm{~s})$ delay is seen when the oven switches from one set temperature to another, during which cooling occurs. It is known that metals start to sinter at $0.5 \mathrm{~T}_{\mathrm{m}}[135]$.

Temperature was recorded at the maximum available sampling rate of every 5 seconds, however there was a display on the device with a live readout of the thermocouple temperature. Occasionally, readings were observed on the live readout which were not recorded by the thermocouple data logger due to the limitations of the sampling rate for the control system. Whilst these values were not recorded, they are mentioned in the following paragraphs as they provide further insight to the temperatures the samples were exposed to.

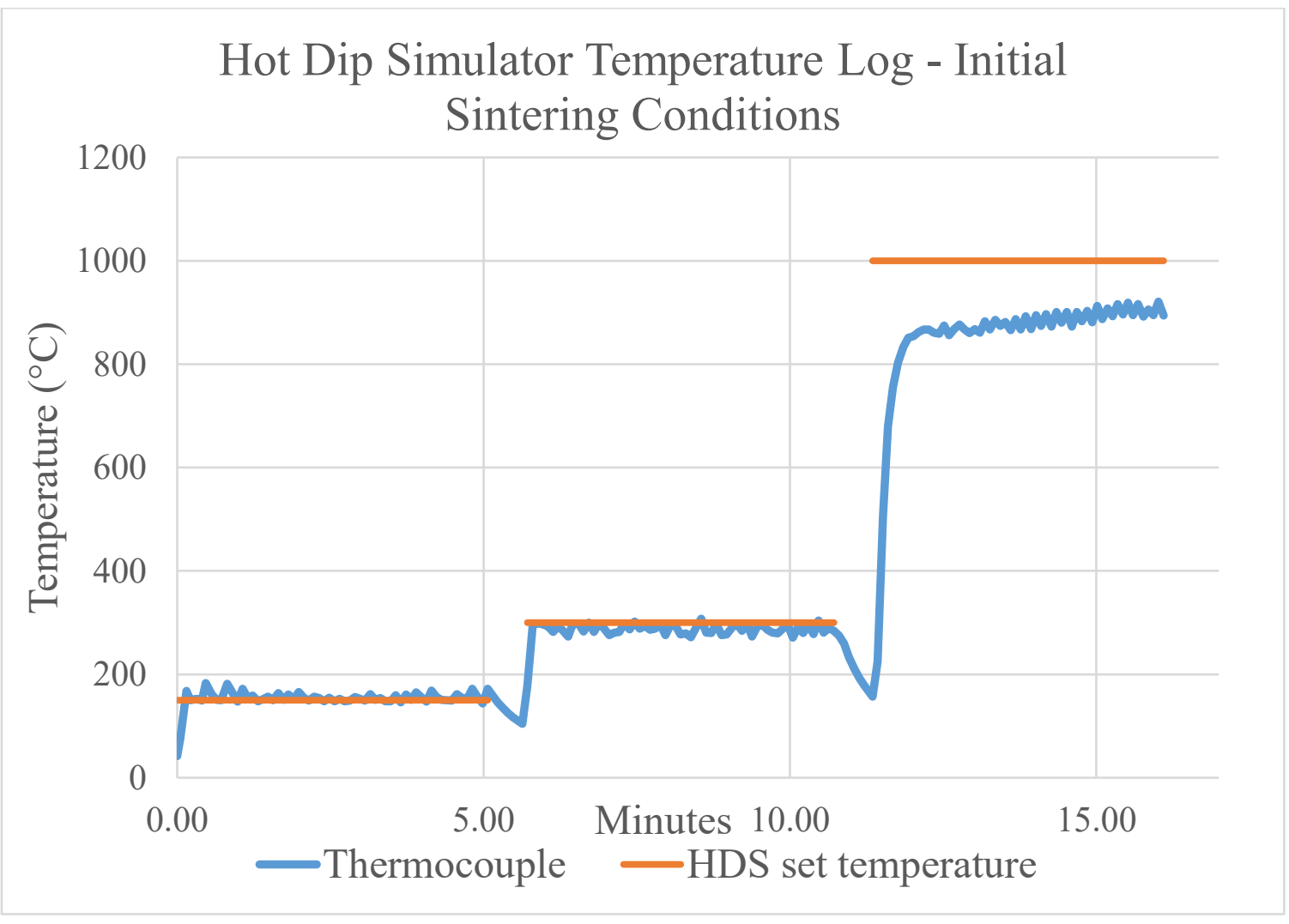

Figure 37 Thermocouple data and HDS programmed set temperature from initial sintering conditions 


\section{Sintering results}

When the programmed temperatures are compared with recorded temperatures in Figure 37 , it is evident that the temperature control of the HDS is poor. When set at $150{ }^{\circ} \mathrm{C}$, the temperature constantly fluctuates between $144-183^{\circ} \mathrm{C}$ (logged temperatures). Likewise, when set at $300{ }^{\circ} \mathrm{C}$, the temperature recorded ranges from $193-308^{\circ} \mathrm{C}$ as the lamps turn on and off. In order to sinter the iron, the HDS was programmed to heat the sample to $1000{ }^{\circ} \mathrm{C}$, however, for reasons of safety, the heating was stopped once the peak metal temperature during operation reached $940{ }^{\circ} \mathrm{C}$ (seen on the live readout). The maximumlogged temperature was $921^{\circ} \mathrm{C}$.

It was noted in Chapter 2 that the HDS uses an aftermarket control system. This uses the temperature measured by the thermocouple to influence the amount of time the lamp stays on. As the thin sheet steel substrate used heats up relatively quickly, the machine cuts power to the lamp after approximately one second of heating, as the control system is trying to avoid the temperature overshooting the set point. Then as the sample rapidly cools, the lamps are turned on again.

This results in a TARDIS like 'pulsing' effect whereby the maximum temperature is lower than the theoretical maximum the machine could operate at for a sample of this thickness. It is theorized that if the control system had allowed for a better control of the infrared lamps, then a significantly higher max temperature would be possible, which would result in reduced sintering times. Additionally, a higher gauge sample may have also resulted in slower temperature increases and would have allowed the control system to input more heat without 'fear' of overshooting the set point. 


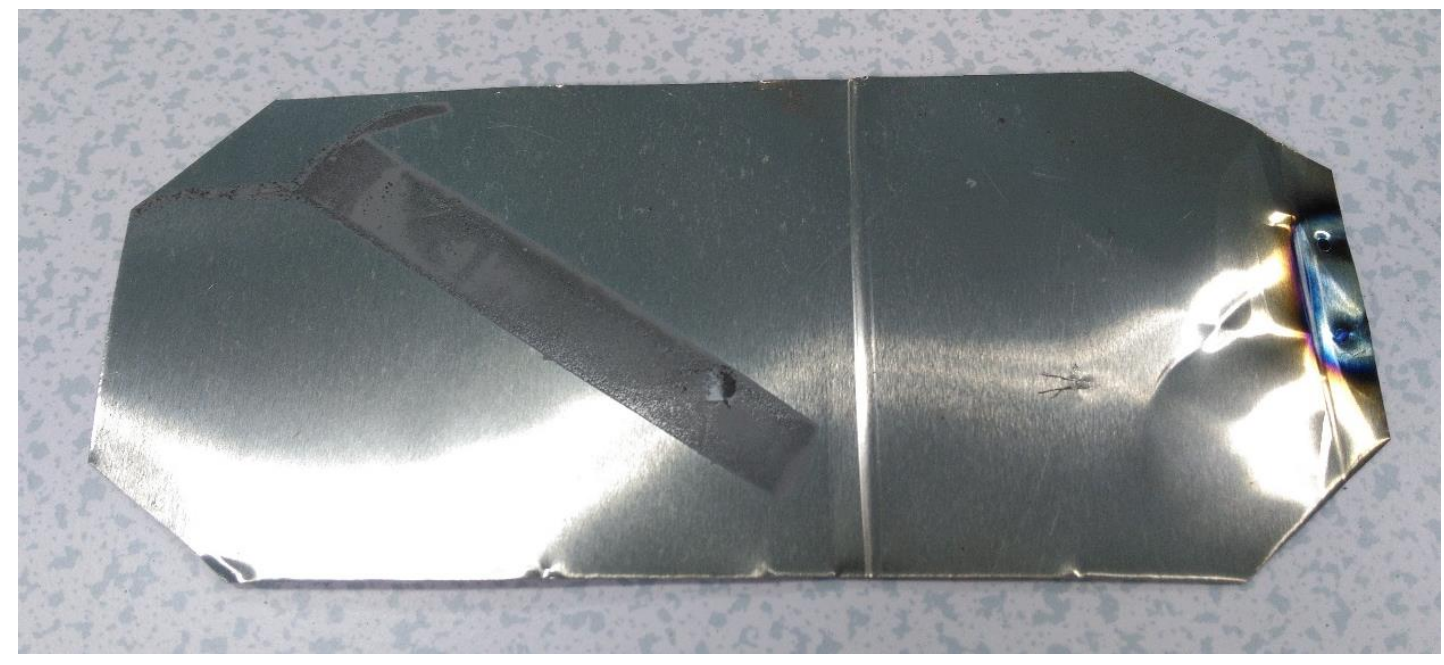

Figure 38 Initial sintering conditions sample (shown after sintering)

The sintered sample is pictured in Figure 38. The area around the sample clamp (right of image) has oxidized slightly as the residual heat in the clamp has caused oxidation after the sample had left the controlled atmosphere section of the furnace.

Figure 38 shows reasonably even heating of the ink across the face of the sample. There is evidence of the ink flowing prior to solvent burn off at the bottom of the sample (left of image - opposite clamp location). 


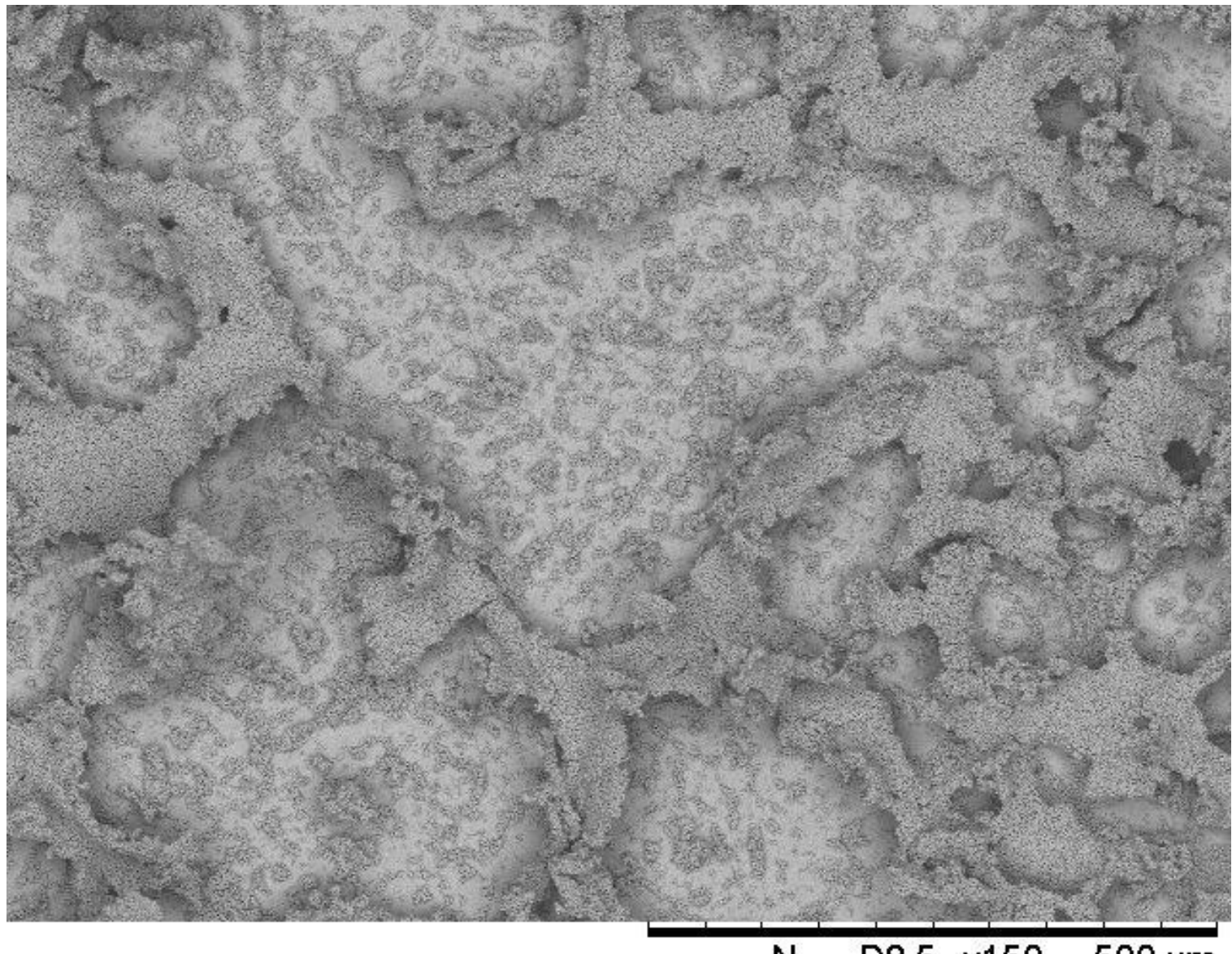

N $\quad$ D8.5 $\times 150 \quad 500$ um

Figure 39 SEM image of sintered iron produced using initial sinter conditions $150 x$

The sample was examined using a Hitachi TM3000 SEM. A varied structure is observed in Figure 39, which appears to show iron forming discrete 'islands', possibly due to convection in the ink prior to solvent burn-off. The substrate is visible and there are small areas of sintered iron which are present on the surface. 


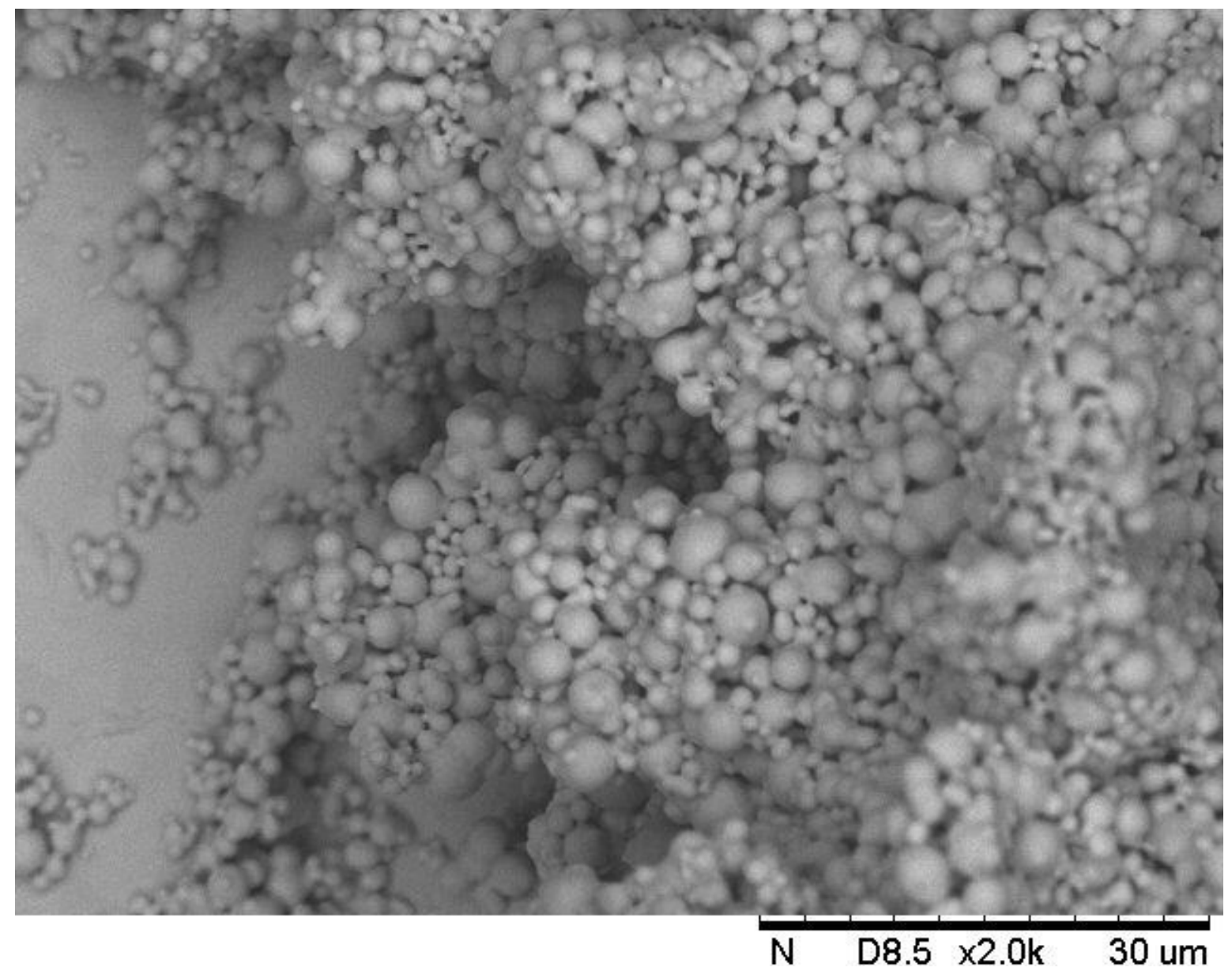

Figure 40 SEM image of sintered iron produced using initial sinter conditions 2000x

Figure 40 shows an higher magnification view of the structure which was present after sintering. It appears that the iron has formed a diffuse structure and the substrate is visible on the left of the image. At this relatively low magnification, it is possible to see necking of iron particles.

Sintering is evident at higher magnification in Figure 41. Particles of iron are sized 3$5 \mu \mathrm{m}$ and show necking on both the smaller and larger particles. This suggests that there is good adhesion within the plaque. 


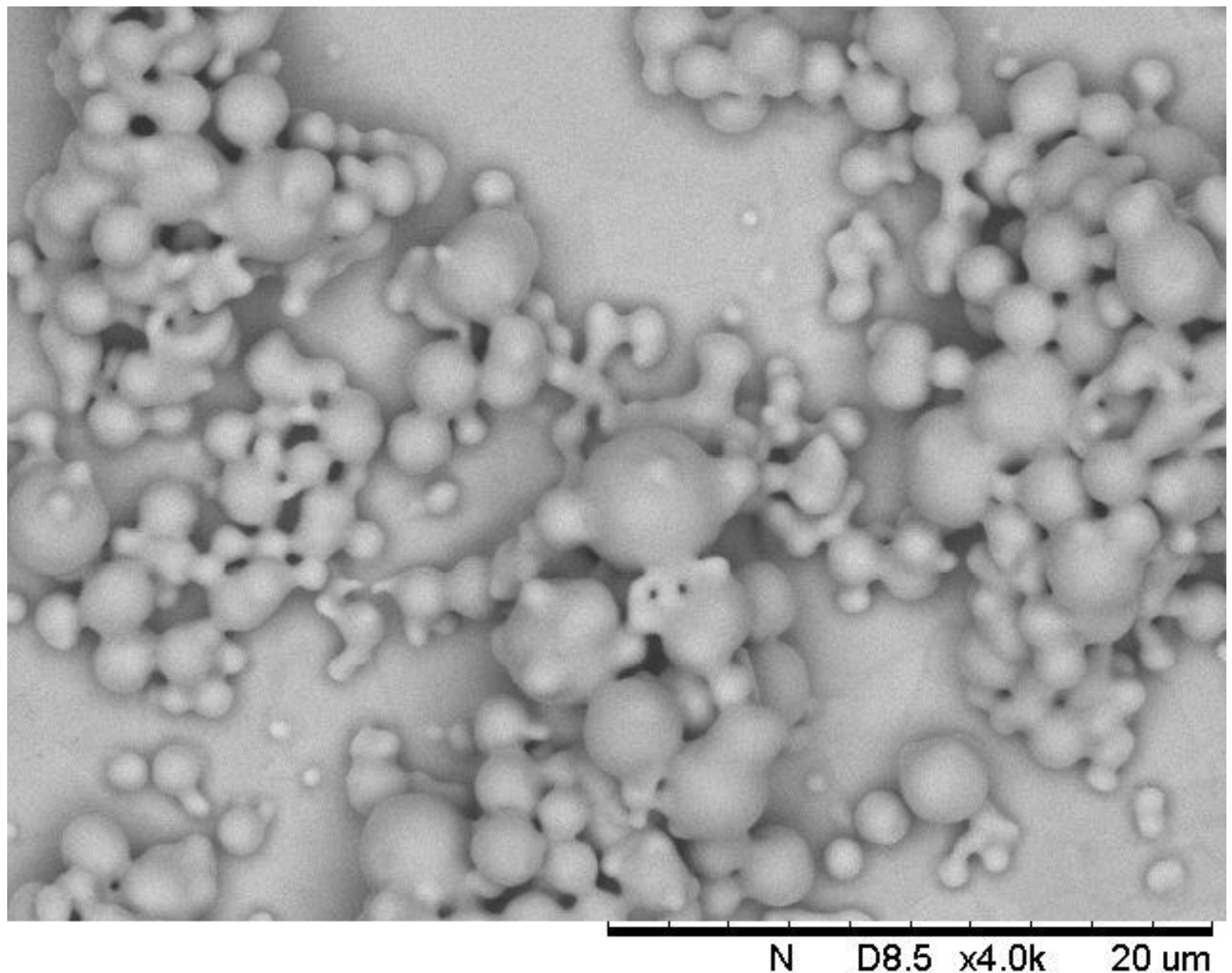

Figure 41 SEM image of sintered iron produced using initial sinter conditions 4000x

This initial sintering test appeared to achieve the desired outcomes. Necking was clearly visible in many areas and no evidence was found of over-sintering, so surface area was not reduced.

It was observed that the coating on this electrode is relatively thin, with only a small amount of active material deposited on the surface. This researcher considered that the amount of active material is small compared to the surface area of the substrate. It is desirable to produce an electrode with a thicker layer of active material to increase the capacity of the electrode and following sections will focus on development of the technique to attempt this. 


\subsubsection{Thicker Electrode}

Following from the success in the above section, here, an electrode with a wet film thickness of $0.26 \mathrm{~mm}$ was produced.

For this test and subsequent tests, the ink was coated as two horizontal strips of size 20 x $65 \mathrm{~mm}$. This allowed for multiple electrodes to be cut from the sample for parallel electrochemical testing.

The heating profile was unchanged from 4.5.1.

\section{Sintering results}

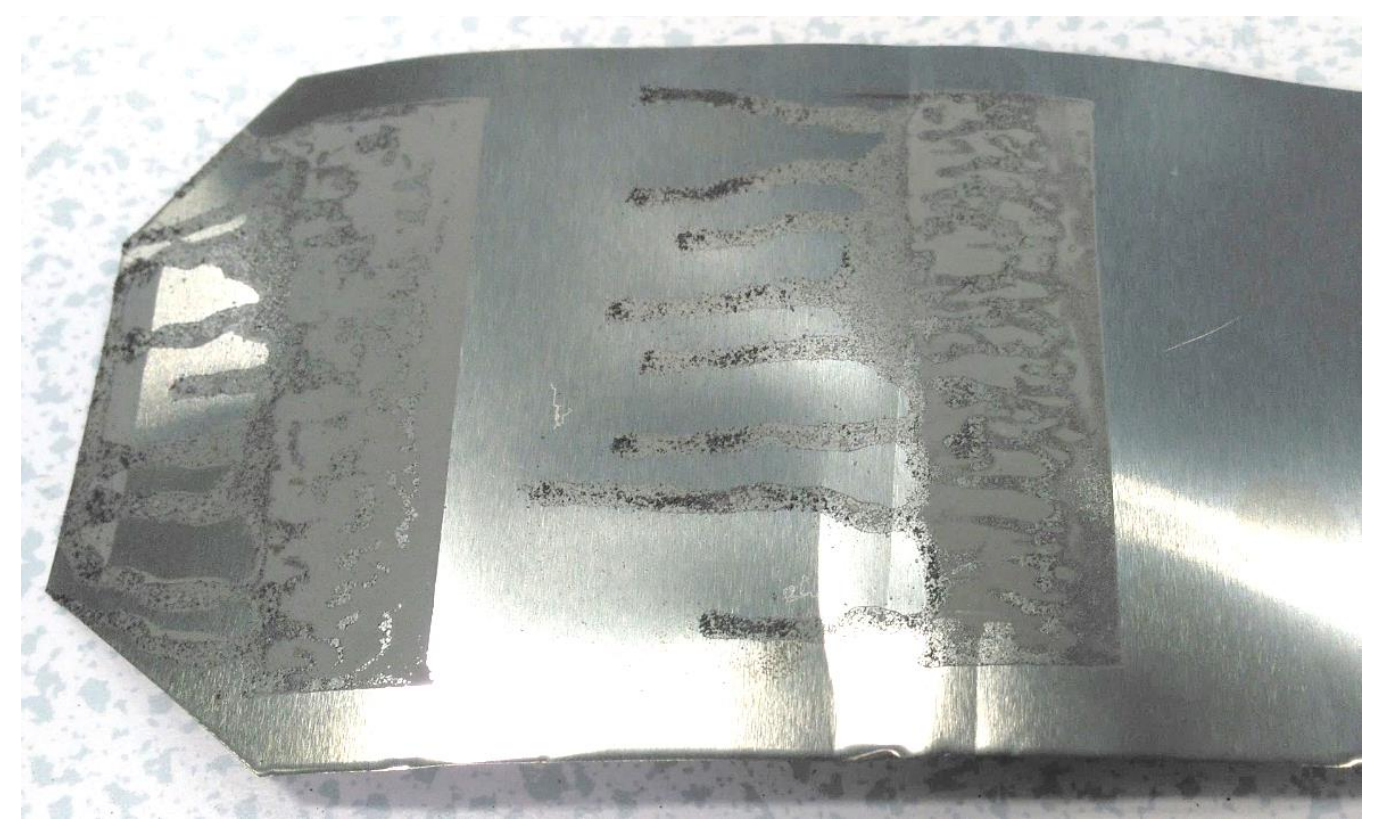

Figure 42 Electrode with thicker layer of active material - evidence of ink running during heating

It is clear from Figure 42 that the ink has flowed with gravity (during heating sample was held vertically from the right of this image). If this method of heating was used in a commercial manufacturing environment, it is likely that the sample would be held horizontally on a conveyer, which would avoid this issue.

As the design of the HDS meant that it was not possible to hold the sample horizontally during heating, it was decided to add a pre-heating step to the process. Using conventional 
heating methods, the ink could be dried without sintering. Adding this extra processing step was undesirable as it makes practical manufacture more complex, however it was deemed necessary in order to produce electrodes with a greater thickness with the equipment available to the researcher.

\subsubsection{Solvent Removal Using a Hotplate}

To avoid the ink running as discussed in 4.5.2, this sample was heated on a hot plate prior to sintering in the HDS. It was anticipated that this would prevent the ink from running as it was heated with the sample held vertically. More rapid heating in the HDS would not have been appropriate, as it would have induced solvent boil.

As previously, the sample was coated with $0.26 \mathrm{~mm}$ of iron-containing ink (4.5.2).

\section{Solvent removal and sintering}

A lab hot plate was used to remove the solvent. It was preheated to $240{ }^{\circ} \mathrm{C}$ and the sample was placed on it. The hot plate was then adjusted to $280^{\circ} \mathrm{C}$ and the sample was left heating for 5 minutes. The increase from $240-280{ }^{\circ} \mathrm{C}$ took approximately 2 minutes. Placing the sample directly on at $280{ }^{\circ} \mathrm{C}$ would have been undesirable as it could have resulted in solvent boil. After 5 minutes, the sample was removed and immediately prepared for sintering in the HDS.

The heating profile for sintering was unchanged from 4.5.1 - sintering was attempted at $1000{ }^{\circ} \mathrm{C}$ for 5 minutes, with heating stopped when a peak metal temperature of $940{ }^{\circ} \mathrm{C}$ was observed on the thermocouple display screen. 


\section{Sintering results}

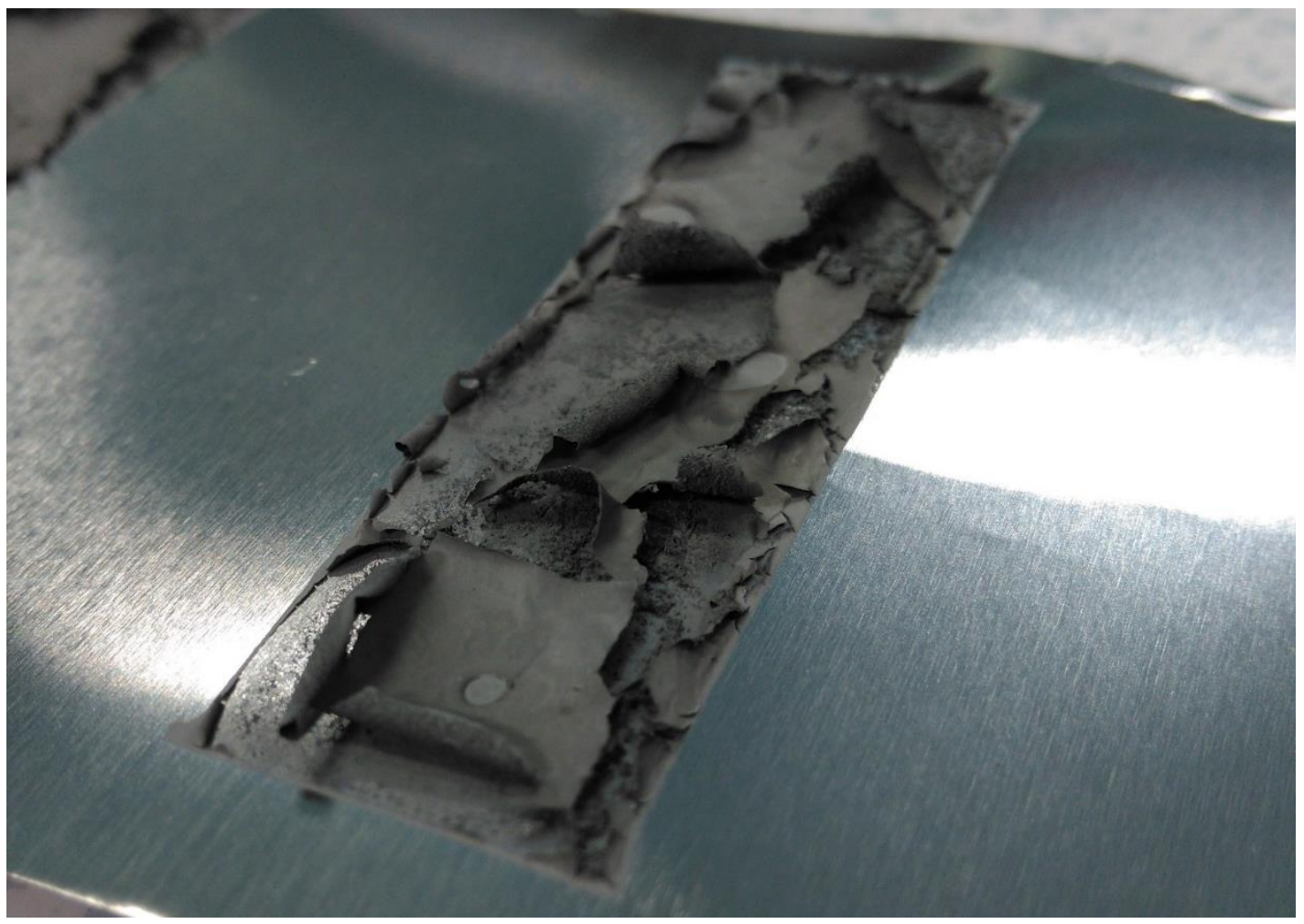

Figure 43 Sample sintered in HDS showing evidence of coating debonding

When the solvent burn-off was completed on the hot plate, the sample did not suffer from the ink running as in 4.5.2. This can be seen in Figure 43, however, the sample has suffered significant debonding. It is thought that this occurred during cooling of the sample after the particles of iron had sintered to form one coherent structure. The curved appearance suggests that the iron has undergone significant shrinkage and thermal stresses under cooling.

Some areas of the coating are missing, suggesting that they have entirely debonded from the substrate and sheared off inside the furnace. It would be impossible to manufacture reproducible coatings if this production issue is not resolved. 


\section{SEM Investigation of Coating Debonding}

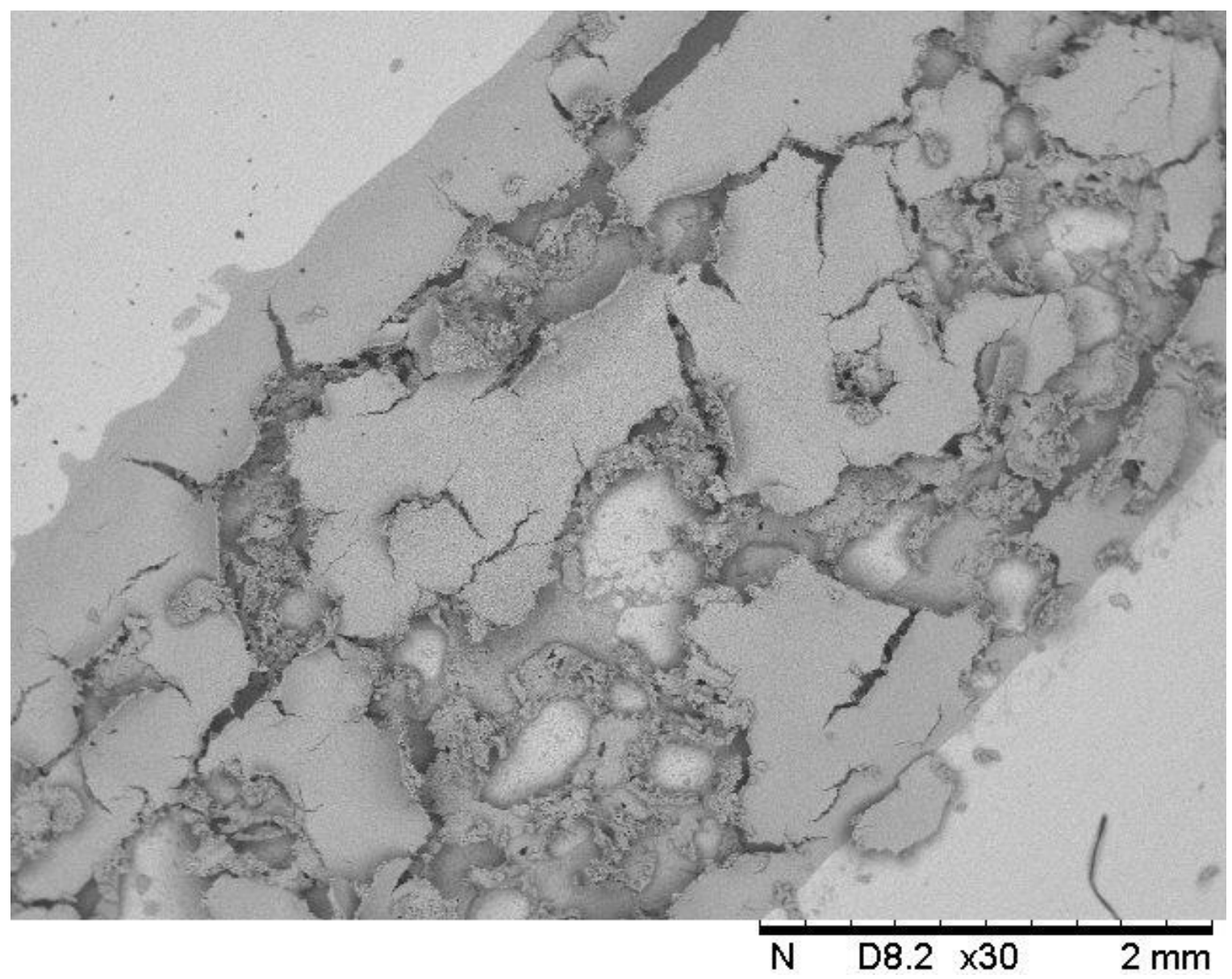

Figure 44 SEM image of sintered iron which has partially debonded 30x

Evidence of partial and, in some areas, complete debonding of the sintered iron coating is visible in Figure 44. It is evident that some areas of the coating are missing entirely.

It is theorized that the thicker coating (vs. 4.5.1) has significantly more mechanical strength and thus does not fragment as readily as a thinner coating under the stresses caused by cooling. 


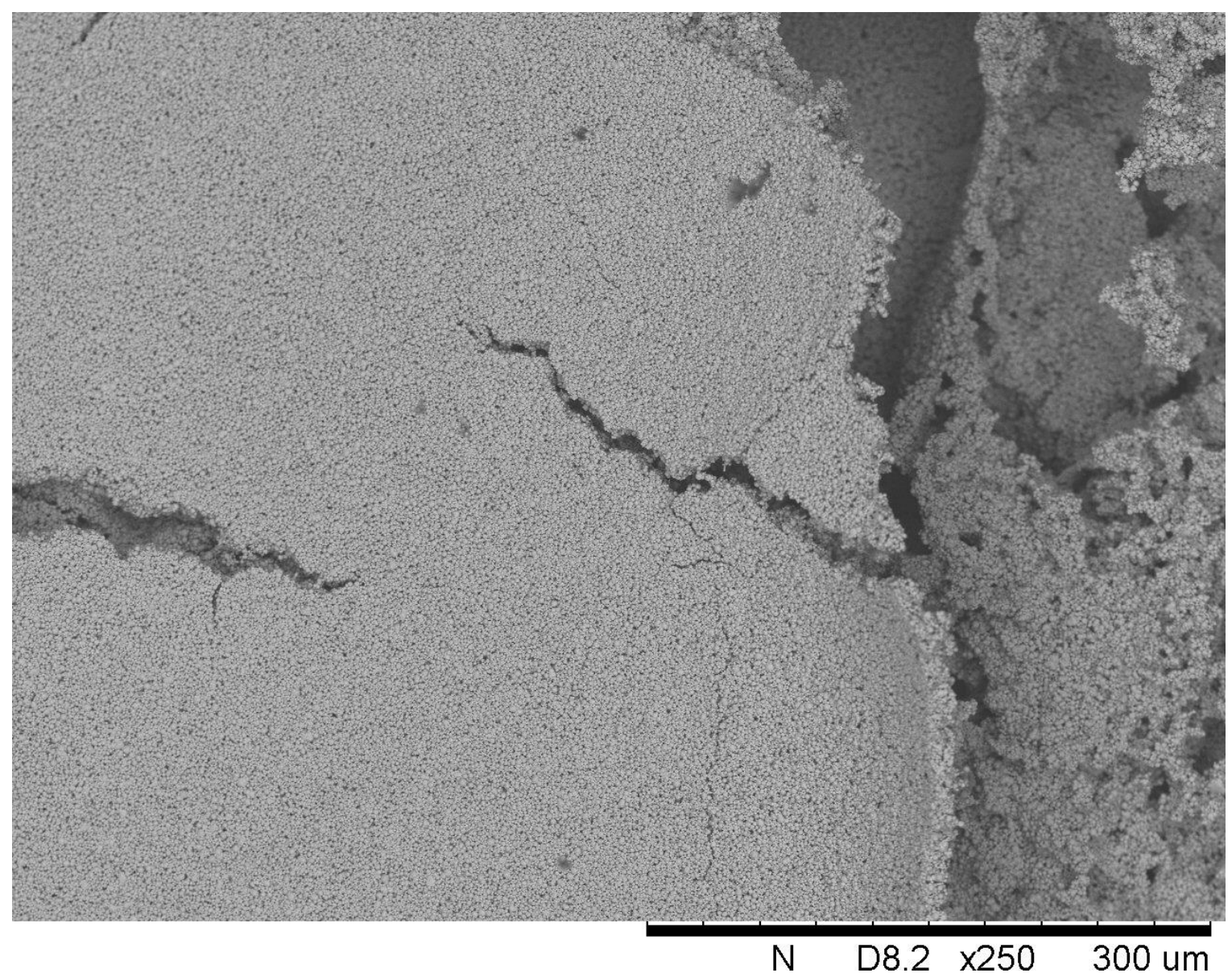

Figure 45 SEM image of sintered iron which has partially debonded 250x

Cracks in the bulk coating are visible in Figure 45. Features like this were seen on many of the large sections of the sintered iron and confirm the previous suggestion that significant shearing forces were present during cooling of the sample.

On the center right of the image it is also evident that the coating is peeling upwards as it shears away from the substrate. 


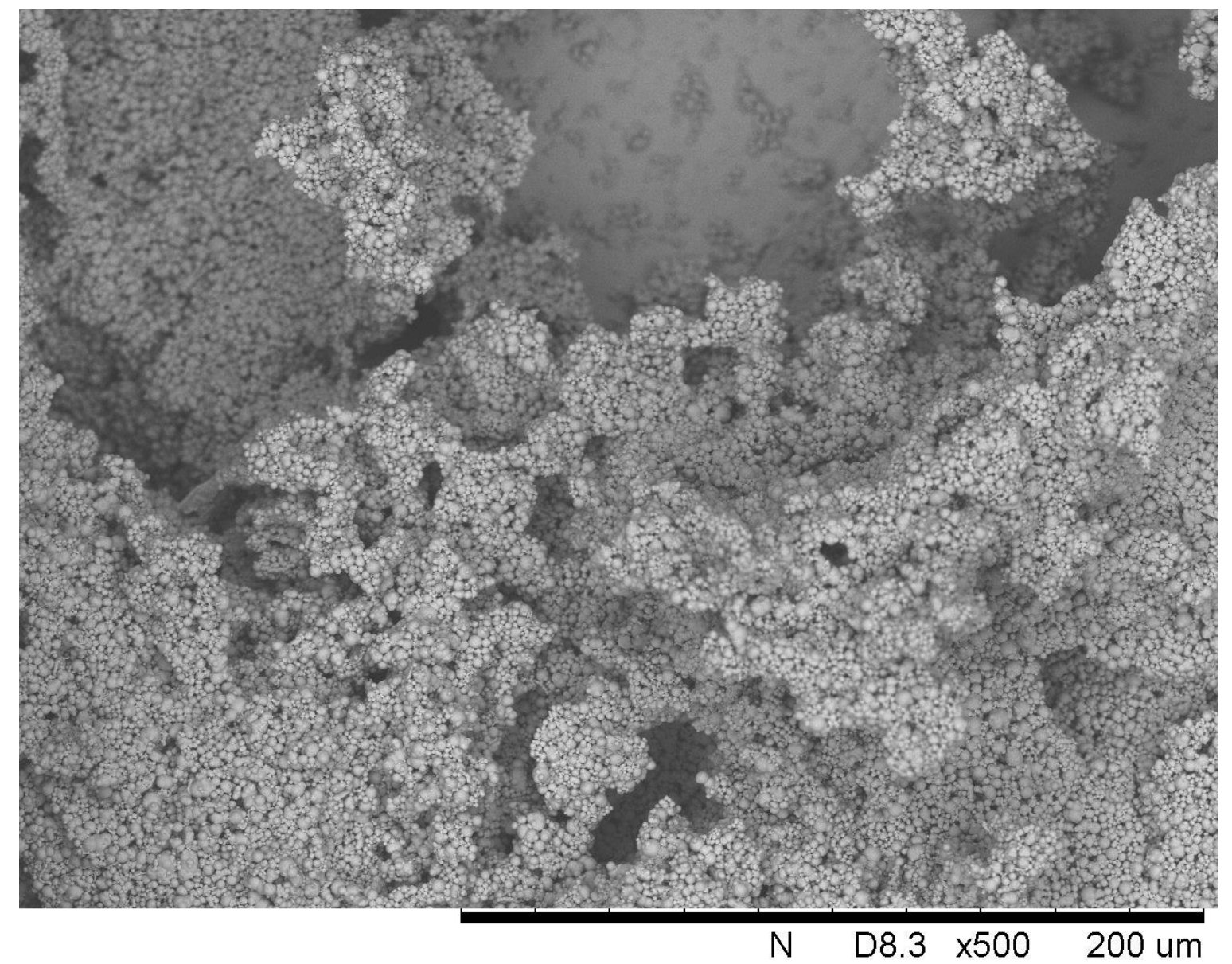

Figure 46 SEM image of sintered iron which has partially debonded 500x

At higher magnification, as seen in Figure 46, there are areas of the sample which appear similar to those seen previously (4.5.1), however, the coating does appear to be thicker. Unfortunately, this is not the case over the entire surface of the sample, due to the areas where the coating has been completely removed, such as at the top of this image.

Further investigation will discuss methods to try to negate the debonding issues so that thicker coatings can be produced in a repeatable manner.

\subsubsection{Oxidation Pre-treatment}

Prior to entering the furnace, the substrate will naturally have a small oxide layer on the surface. It is considered that the reducing atmosphere in the furnace may be unable to fully reduce this oxide layer and this may be a factor affecting the adhesion of the iron coating to the substrate. Somewhat counterintuitively, it is reported in literature that 
increasing the thickness (or oxidation state) of the surface oxides on the substrate may result in more complete reduction and subsequently, better adhesion of the sintered iron coating to the substrate [190], [199].

When used industrially, the process typically involves intentionally oxidizing the substrate in a controlled environment, prior to reducing the oxide back to iron [200]. This is reported to improve surface wettability - as there is less oxidation present on the substrate surface post-reduction than if the oxidation pre-treatment had not occurred [201].

It is not known whether incomplete reduction was an issue in the particular case discussed previously, however, an oxidation pre-treatment is examined to test its effects.

\section{Pre-treatment method for growing oxide on substrate}

To grow the oxide layer on the substrate, prior to coating the ink, a small section of the substrate was heated on a hotplate in air to $400{ }^{\circ} \mathrm{C}$ until a brightly colored oxide layer could be seen (Figure 47). This operation took approximately 4 minutes.

The sample was allowed to cool prior to coating with $0.26 \mathrm{~mm}$ thickness of iron ink over the oxidized area, in the manner described in 4.5.2.

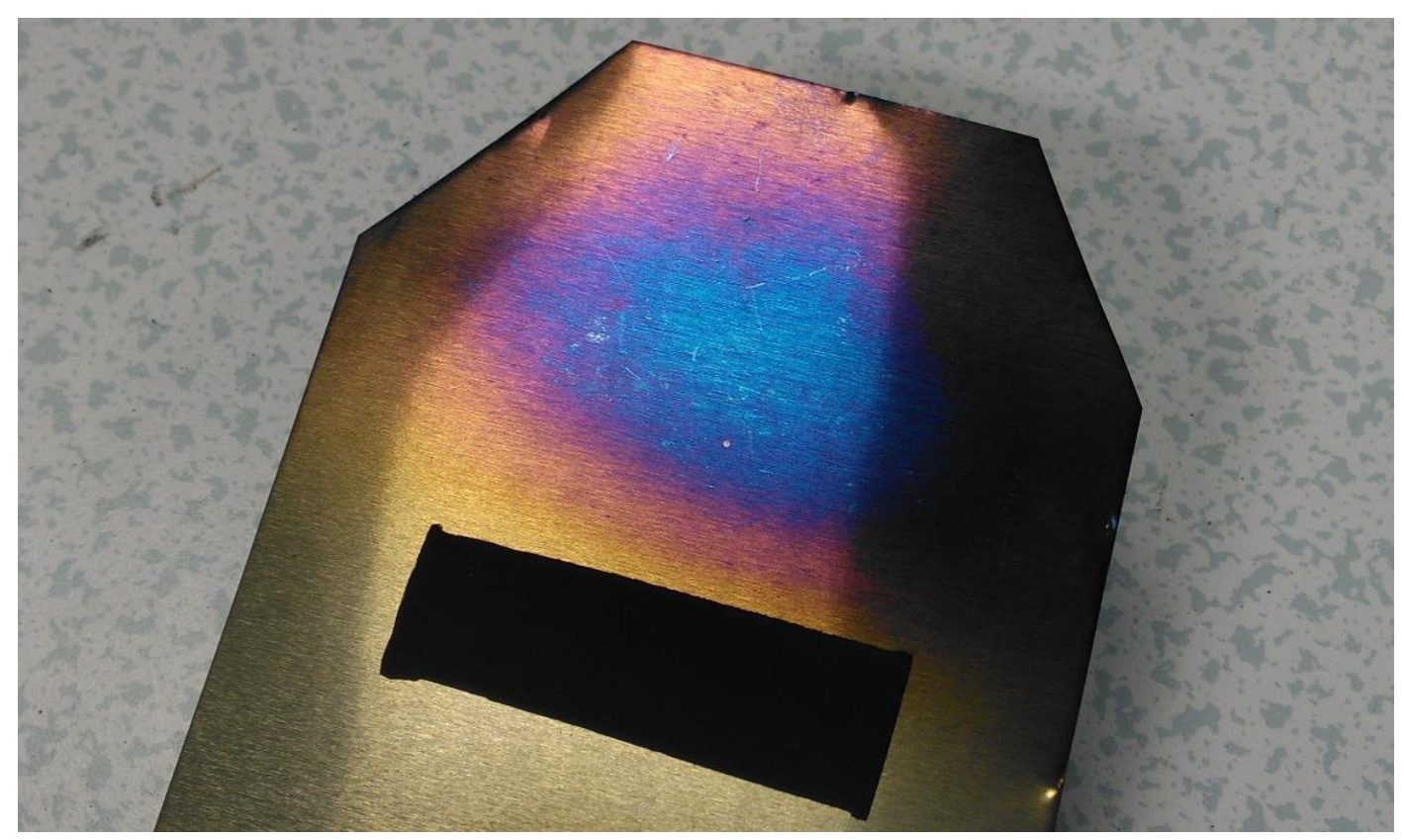

Figure 47 Oxidized substrate 


\section{Solvent removal and sintering}

Performed as in 4.5.3.

\section{Sintering results}

Figure 48 shows coating debonding over the area which was oxidized in the pretreatment. From this it was determined that coating adhesion was not improved by this pre-treatment method. This could be because the thicker oxide layer was impermeable to the reducing gasses and was not sufficiently reduced.

If insufficient reduction of the oxides on the substrate is the reason for the poor adhesion of the coating to the substrate, then the failure of the extra oxidation step to result in a more complete reduction would result in this pre-treatment not providing a beneficial effect. It was decided not to continue with oxidation pre-treatment. An alternative approach where oxide film was removed prior to coating was investigated.

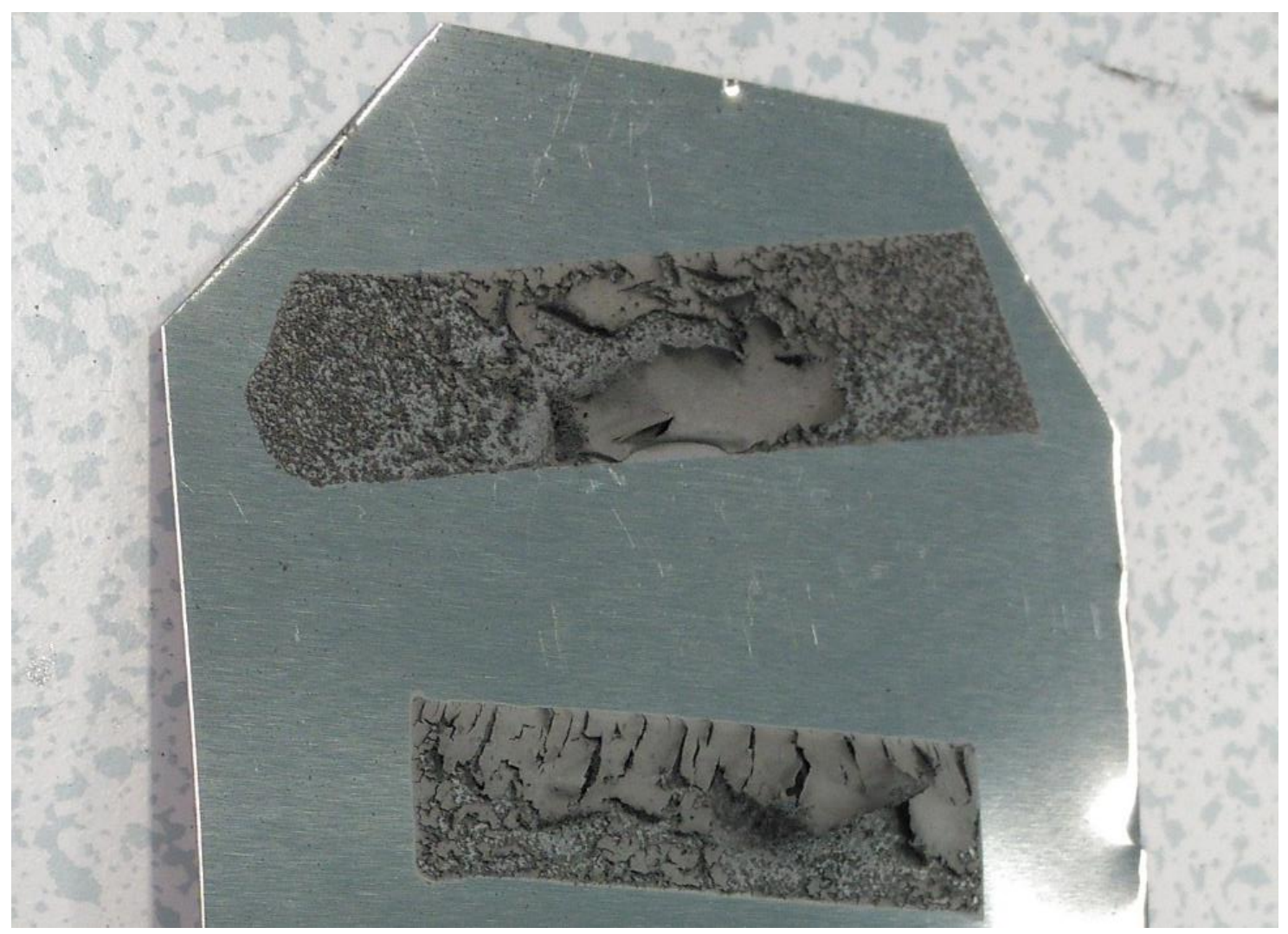

Figure 48 Oxidised pre-treatment after sintering in HDS 


\subsubsection{Surface Oxides Removed by Pre-treatment}

As identified previously, it was considered that the surface oxide film on the surface of the substrate could be a barrier to reduction in the HDS and therefore may inhibit metallic bonding and adhesion of the iron coating. Whereas in 4.5.4 the substrate oxide layer was increased, here attempts are made for it to be removed. If adhesion is related to the surface oxide then this is a logical step to attempt to resolve the issue or eliminate it as a potential factor.

\section{Pre-treatment method for removing surface oxides}

Steps were taken to remove this oxide film prior to coating and heating of the sample. This was achieved by abrasion of the substrate with a P1200 Silicon Carbide Abrasive Sheet (3M).

This operation was completed in the clean room (Class 10,000, ISO7) in the Pilot Manufacturing Resource Centre, Swansea University, due to its reduced humidity. This allowed the surface to be abraded to remove surface oxides and then immediately coated without excessive corrosion occurring and the oxide reforming. Immediately following this oxide removal, the $0.26 \mathrm{~mm}$ coating was applied as before.

It is noted that 'pickling' the substrate with a strong acid would have been an alternative method of oxide removal.

\section{Solvent removal and sintering}

Performed as in 4.5.3.

\section{Sintering results}

No improvement to the adhesion or structure of the coating was found despite this pretreatment to remove surface oxides on the substrate. The coating was similar in appearance to previous failed coatings; however, no image is available. 
It is not known if the abrasion prior to coating was effective at removing oxides. The rate of surface oxide regrowth in the reduced humidity environment of the clean room was also not known.

Pickling the substrate with hydrochloric acid or sulfuric acid was considered as an alternative method of oxide removal. However, considering that the previous efforts to enhance the oxide or remove the oxide have failed to impact the adhesion of the coating, it is unlikely that further modifications to the oxide layer would have a positive impact on coating performance. Alternative methods are considered.

\subsubsection{Reduced Cooling Rate}

In an effort to avoid debonding occurring as a result of thermal stress, the cooling rate post-sintering was reduced. This would result in reduced thermal stresses acting on the sintered iron, similar to an annealing step, which would mean that the coating would not shear off the substrate during cooling. This would only be affective if this is the mechanism of debonding.

Typically, once the sintering temperature is reached, the furnace no longer heats the sample. As the furnace is surrounded in a water cooling 'jacket' and the substrate has limited thermal mass, heat is lost very quickly after the heating lamps turn off. For this reason, additional heating steps were manually added to effectively slow the cooling rate of the sample. This was achieved by applying heating in stages after sintering had completed.

\section{Solvent removal and sintering}

A sample was coated and heated on a hot plate as previously described in 4.5.3.

Sintering was again programmed to heat to $1000{ }^{\circ} \mathrm{C}$ for 5 minutes, however heating was again stopped when maximum temperature of $940{ }^{\circ} \mathrm{C}$ was observed on the thermocouple display screen. This discontinuation temperature was set to ensure repeatability with previous tests. 
After sintering, the furnace was programmed to heat to $800{ }^{\circ} \mathrm{C}$, then $600{ }^{\circ} \mathrm{C}$ and finally $400{ }^{\circ} \mathrm{C}$ before turning off and cooling naturally, as shown on Figure 49. These cooling plateaus were not set to automatically progress on to the next step, but instead to halt until the operator progressed the step. This was to avoid excessive use of the reducing gas whilst ensuring that the furnace had settled at the desired temperature prior to moving on to the next set point.

\section{HDS temperature profile}

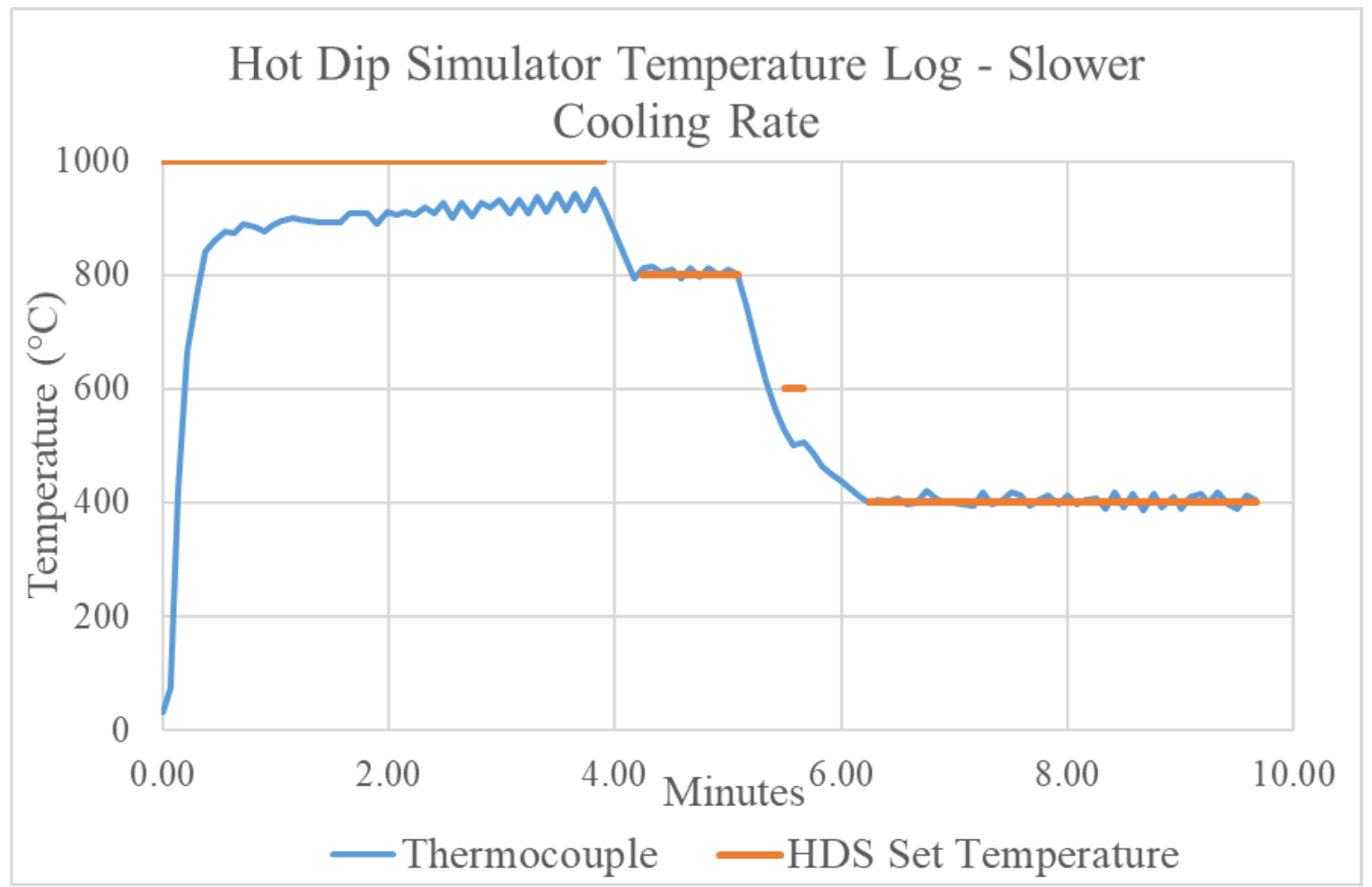

Figure 49 Temperature log from HDS with reduced cooling rate

It is evident from Figure 49 that the cooling profile attempted was not completed successfully. The aftermarket control system (2.1.4) appeared to be faulty and would not reliably interpret requests to heat to a temperature lower than the current temperature. This test was repeated (not shown) and the same issue occurred.

This resulted in the temperature not 'plateauing down' as desired. The $800^{\circ} \mathrm{C}$ plateau was followed, but the HDS appeared to skip the $600{ }^{\circ} \mathrm{C}$ step and move immediately on to the $400{ }^{\circ} \mathrm{C}$ step. 
Nevertheless, the cooling rate of the sample post-sintering was significantly decreased and this effect was considered sufficient to examine the sample.

\section{Sintering results}

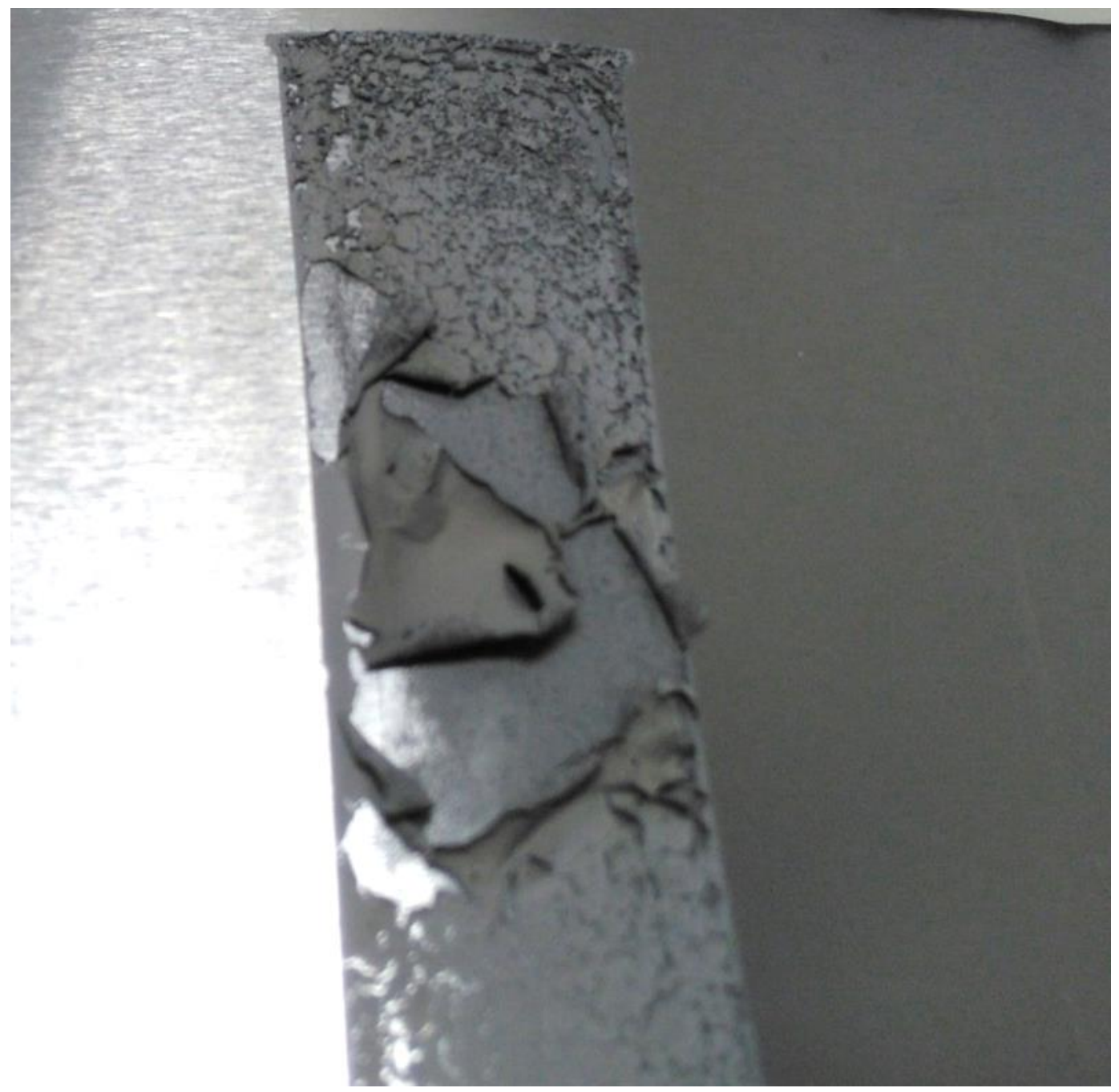

Figure 50 Sintered sample which had been cooled at a slower rate

Blistering and coating debonding was still present on this sample, as evident in Figure 50. The attempt to prevent coating debonding by reducing the cooling rate to reduce the thermal stress on the coating was not successful.

It is noted that if it had been deemed safe to disable the water-cooling on the HDS furnace, this could have provided a significantly slower and more reproducible reduced cooling rate. 
An electric tube furnace would have been an alternative to provide a further reduced cooling rate; unfortunately, this equipment was not available to the researcher with the required reducing gas atmosphere to avoid oxidation of the sample.

\subsubsection{Increased Cooling Rate}

Previous efforts to form a stable and repeatable electrode were unsuccessful and a reduced cooling rate had not produced a stable coating. An alternative method was investigated: increasing the cooling rate to intentionally induce brittle fracture in the coating. Ideally, this would encourage the coating to 'shatter' in to many smaller pieces. It was hoped that this would reduce the tendency of the coating to shear off the substrate. Smaller fragments of the coating would have reduced mechanical stress during cooling.

This was achieved by increasing the cooling rate in the furnace after completion of the sintering step. The nitrogen jets were activated immediately after sintering to provide a nitrogen 'quench' until the sample had cooled to room temperature. Whilst this would dilute the reducing properties of the $5 \%$ hydrogen atmosphere used during sintering, it was not expected to result in significant oxidation of the sample as the atmosphere will continue to be inert.

\section{Solvent removal and sintering}

The ink was dried on a hot plate and sintering was performed as described in 4.5.3.

After sintering, jets of nitrogen were activated to rapidly cool the sample. Heating was discontinued and the water jacket remained on.

Unfortunately, due to limitations of the aftermarket control system, as the furnace was not heating during the nitrogen-quenching phase, it was not possible to record the thermocouple temperature. As a result, it is not possible to compare the cooling rate from the previous experiments.

The nitrogen quench was discontinued when the sample had cooled below $100{ }^{\circ} \mathrm{C}$ as visible on the live readout on the thermocouple display. This took approximately 30 seconds. 


\section{Sintering results}

Sintering with rapid cooling (nitrogen quench) resulted in a stable and repeatable coating, as evidenced in two separate samples on different substrates in Figure 51.

Only minimal debonding of the iron coating was observed. The majority of the coating appeared to be present and had fractured into discrete islands of size $<5 \mathrm{~mm}^{2}$. It is thought that this fracturing was due to the increased cooling rate, which was achieved through the use of the nitrogen quench.

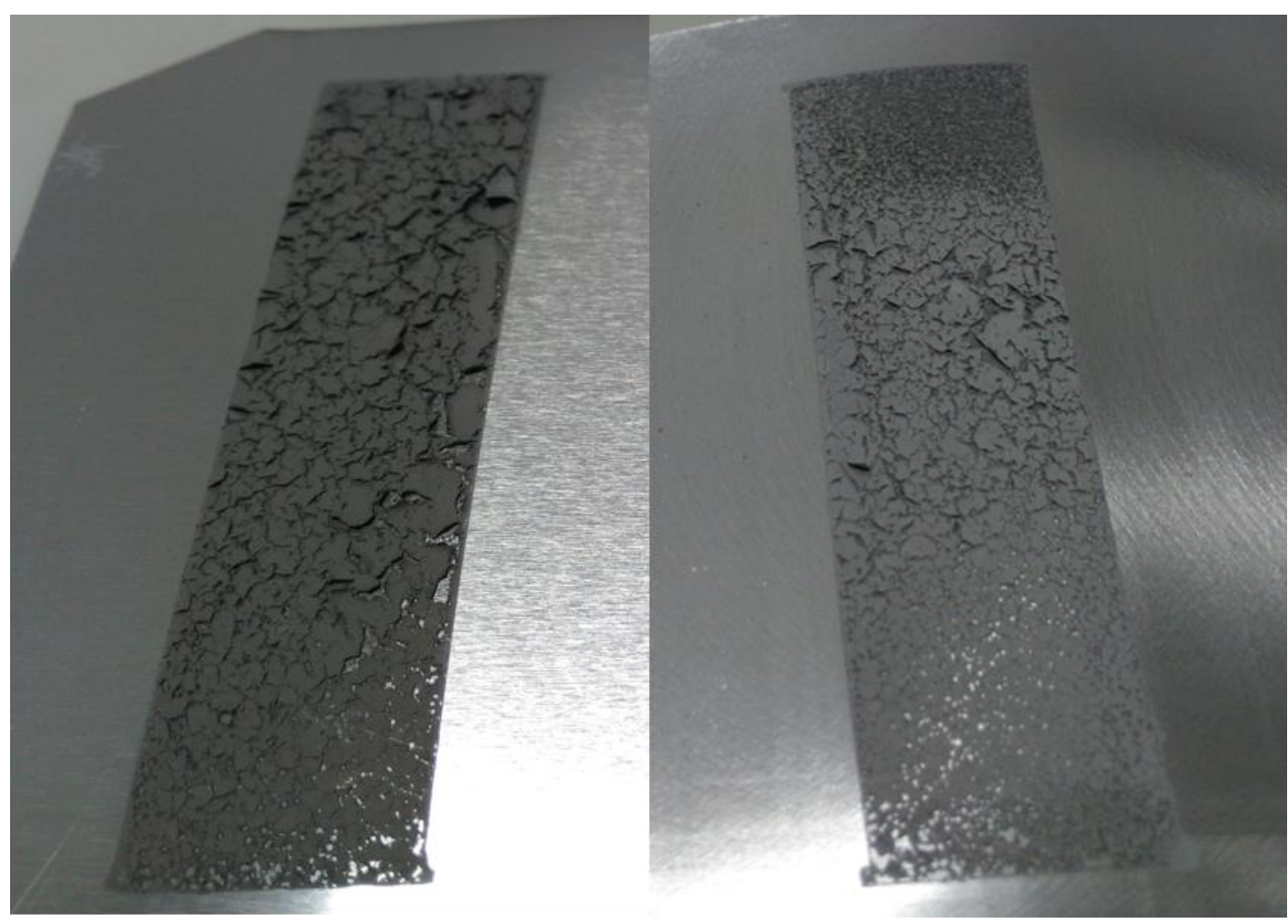

Figure 51 Iron coated samples sintered with increased cooling rate (nitrogen quench)

\subsection{Sintered Electrodes for Electrochemical Testing}

Sintering, as described in 4.5.7, was considered to be successful. Previously, electrodes produced using the low temperature production route and a PTFE binder were manufactured on a range of substrates (Chapter 3). Likewise, sintered electrodes were also produced on a range of substrates to see if the adhesion was affected. 
The following substrates (2.1.1) were used: as received, wire brushed, ground.

Electrodes were produced using the 'nitrogen quench' method as described 4.5.7, with sintering being completed in 5 minutes.

\subsubsection{Electrochemical Testing of Sintered Electrodes}

Here, electrochemical testing of electrodes produced in 4.6 is described.

As mentioned previously (4.2), these tests were not intended to assess the performance of different electrodes/substrates, but instead to assess the mechanical stability of the electrodes and their potential suitability for further investigation. Electrodes were tested using a 64 channel Arbin SCTS as described in 2.1.5. Nine cells were tested in total, three repeats for each substrate used.

\subsubsection{Results and Discussion}

All cells cycled successfully without any noticeable delamination or breakdown of the electrodes.

A theoretical capacity of each electrode was calculated based on the calculated mass of active material present. This was used to produce a utilization (Figure 52) based on the calculated theoretical capacity of the electrode and the measured discharge capacity on the $55^{\text {th }}$ cycle. The $55^{\text {th }}$ cycle was selected as it was expected it would exclude the variability that would occur during the initial formation cycling of the cell, but meant the experiment wasn't excessively long. 


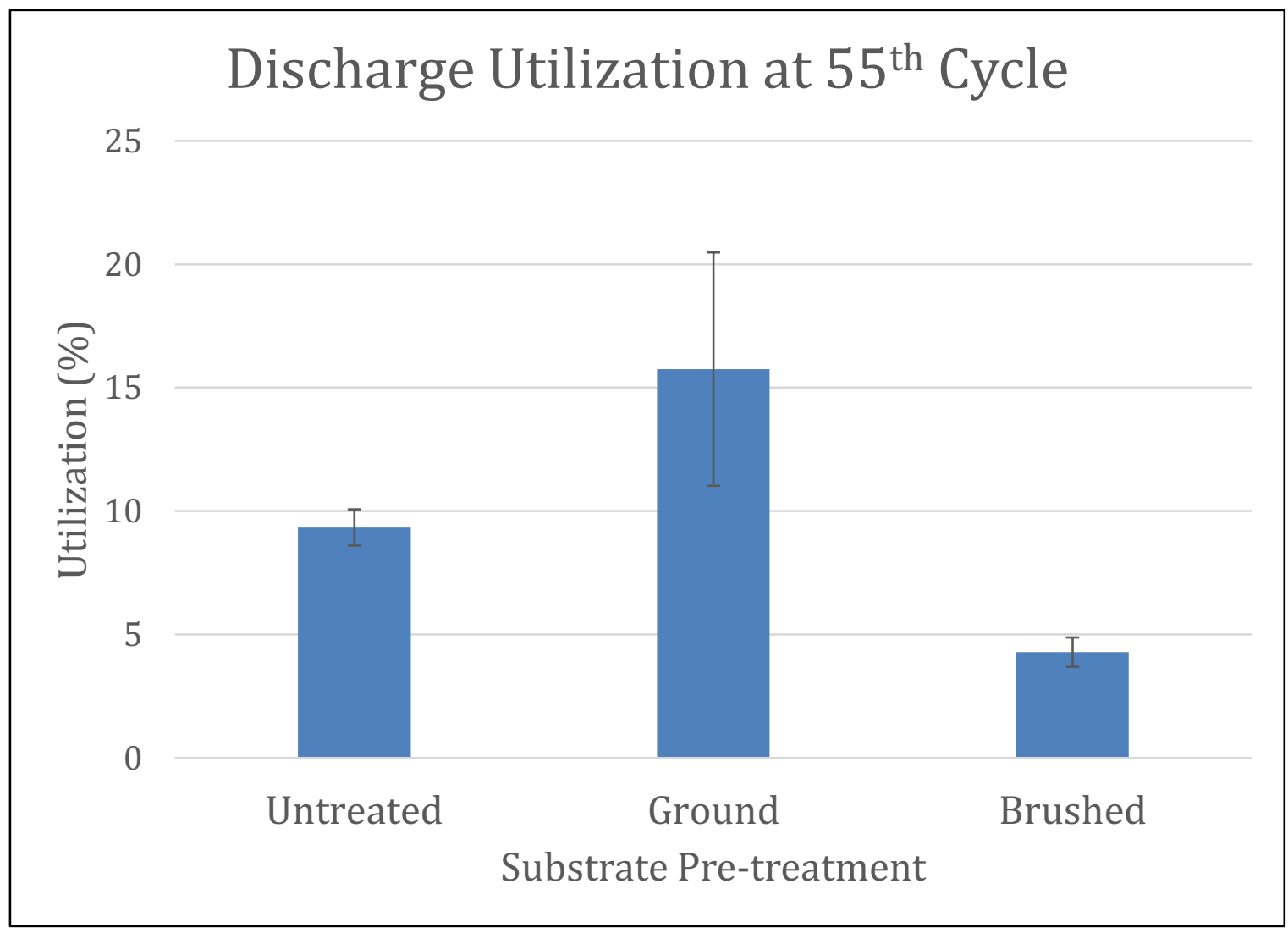

Figure 52 Average percentage utilization of sintered iron samples (error bars show standard error)

Utilization of the theoretical capacity of active material was low, however, these electrodes did not employ any blowing agents or pore formers to improve porosity, so much of the mass of active material would be inaccessible and unavailable for reaction. Whilst surface area was not measured, it is expected that it could have been improved with blowing agents or pore formers.

It is noted that the standard error is particularly high in the case of the sample with the ground substrate. This is partially due to the small data set ( 3 samples), but also due to the sample which performed particularly well (Ground A) as seen on Table 3. 
Table 3 Utilization of sintered iron samples coated on to various substrates $\left(55^{\text {th }}\right.$ cycle)

\begin{tabular}{|c|c|c|c|c|c|}
\hline \multirow{2}{*}{$\begin{array}{c}\text { Substrate Pre- } \\
\text { treatment }\end{array}$} & \multicolumn{3}{|c|}{ Raw Utilization\% } & Utilization\% & $\begin{array}{c}\text { Standard } \\
\text { Error }\end{array}$ \\
\cline { 2 - 5 } Untreated & 8.73 & 12.13 & 7.15 & 9.34 & 1.47 \\
\hline Ground & 34.59 & 5.01 & 7.65 & 15.75 & 9.45 \\
\hline Brushed & 6.62 & 2.76 & 3.48 & 4.29 & 1.18 \\
\hline
\end{tabular}

The cycling performance of the 'Ground A' sample is seen in Figure 53. This shows formation is continuing up to the end of the dataset, evident by the discharge capacity continuing to improve throughout the test. Performance may have improved further if cycling was not discontinued.

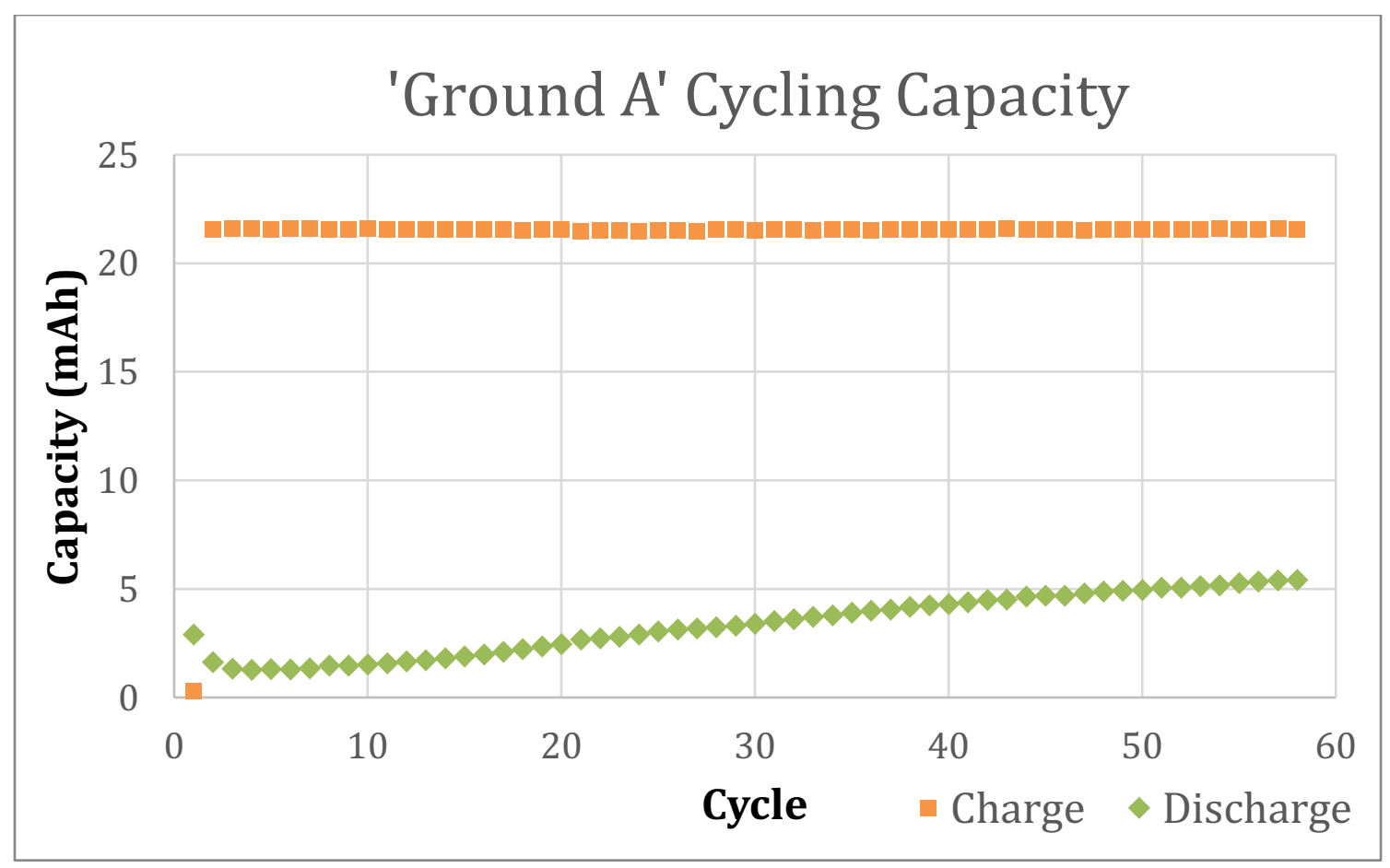

Figure 53 Charge and discharge capacity attained in cycling of sample 'Ground $A^{\text {' }}$

Whilst the performance of the 'ground A' sample was superior to the other samples, it is not thought that the sample was better due to a structural/physical difference in the electrode. It is thought that the $\mathrm{C}$ rate used was more appropriate or accurate for this particular electrode, resulting in the sample performing closer to its theoretical maximum 
practical performance. Accurately calculating the theoretical capacity used to program the battery tester is difficult, as allowances need to be made for the availability of the active material deposited. This researcher found that, particularly with small samples, minor alterations to calculations could make the difference between and effective charge and an ineffective charge. Given the manufacture and testing of all electrodes was controlled to be equivalent, excluding the defined variable, it is possible that the charging conditions were most appropriate for the 'ground A'. It is considered that with a more advanced charging control protocol, such as constantly adjusting charge rate based on performance over the last cycle, equivalent performance could have been attained from all cells.

For electrodes other than 'Ground A', the C rate may have been too high and thus more aggressive, resulting in poor charging and discharging performance, with only small quantities of active material undergoing oxidation or reduction. This would result in very poor utilizations. Equally, the $\mathrm{C}$ rate could have been too low, resulting in the time limit for the $0.2 \mathrm{C}$ charging elapsing long before the required voltage limit was reached. The effect of this would be limited charge storage and therefore a poor discharge capacity attained, with the result being an ineffective formation.

The range seen between each cell could have also resulted from variability in the manufacturing process. Possible causes could be: the coating method used and poor coating technique; improper mixing of the ink; local variations in heating conditions or variations in the counter electrodes used. For example, it would be expected that, due to a larger surface area, the cells containing an electrode manufactured on a brushed substrate would outperform the untreated samples, however this is not observed in Figure 52. It is possible that this substrate performs poorly as an electrode substrate, however it is also possible that the differences are a result of manufacturing characteristics or charging conditions (poor match between $\mathrm{C}$ rate and cells). 


\section{Typical Cycling Voltage Profile}

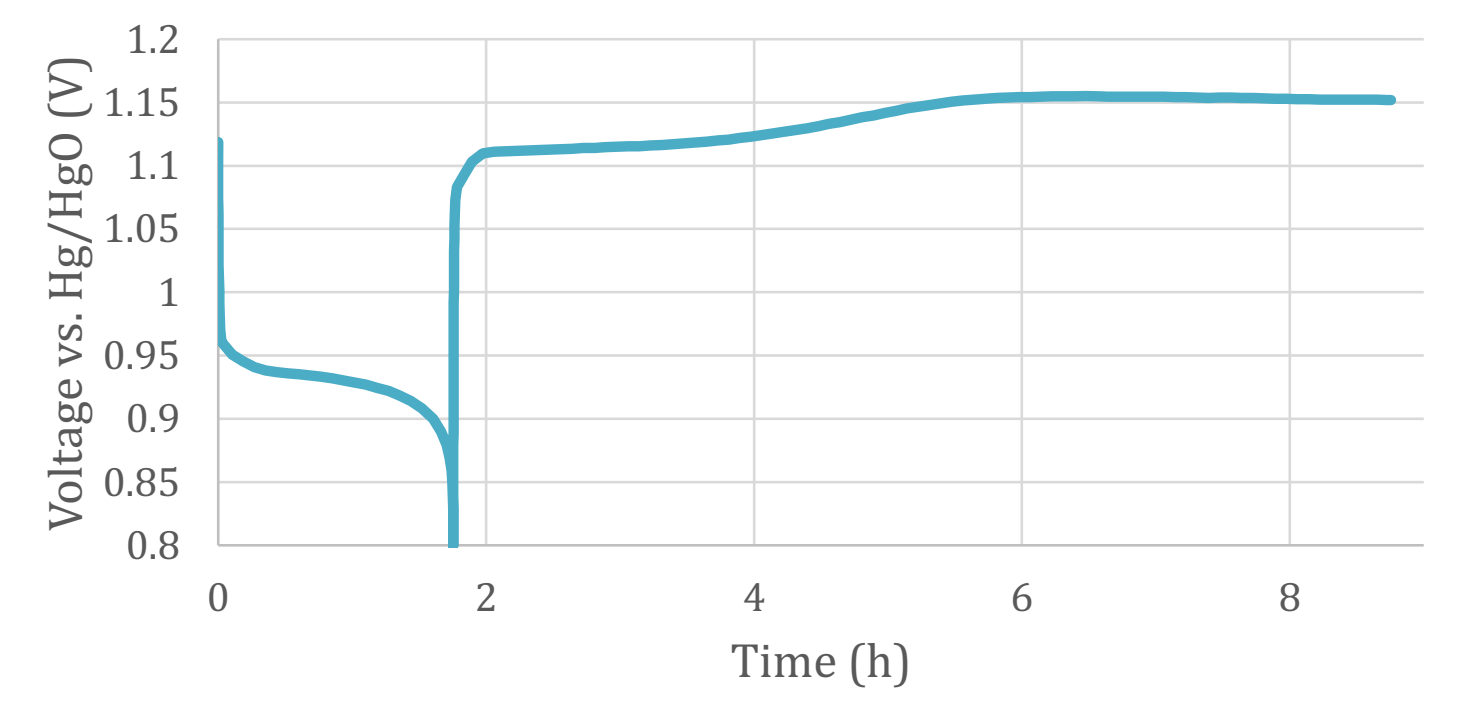

Figure 54 Discharge/charge voltage profile vs. $\mathrm{Hg} / \mathrm{HgO}$ reference during electrochemical cycling

Regardless of the above-discussed difficulties, the voltage profile of the electrochemical testing (Figure 54) shows reasonable agreement with literature [53], [111]. Discharge may have been more effective and thus utilization and columbic efficiency improved, if discharge was continued down to $0.6 \mathrm{~V}$, however this would have resulted in oxidation of $\mathrm{Fe}^{2+}$ to $\mathrm{Fe}^{3+}$ which is known to be detrimental [135].

On the basis of these results, it is determined that the electrodes manufactured in 4.6 are of sufficient mechanical stability to withstand cycling, as no electrodes delaminated. Detailed comparison of electrode stability or further analysis of electrochemical results is not detailed here as it is outside the scope of this work.

\subsection{Conclusions}

In this chapter, sintered electrodes have been produced using a hot dip simulator as an IR furnace. As discussed in Chapter 3, printed electrodes offer the potential for advantages in cost, which translates to lower cost over the life of a battery. Sintered electrodes may perform better and have longer lives than electrodes which have been produced with binders. 
After the lengthy iterative process described in 4.5, suitable conditions for producing sintered iron electrodes were identified.

Electrodes were then produced with a wet film thickness of $0.26 \mathrm{~mm}$ on the following substrates: as received, ground or brushed. Sintering was performed as described in 4.5.7 and was achieved in approximately 5 minutes, significantly faster than that reported in literature (1.6.4).

Electrochemical testing was performed on the resultant electrodes, with results detailed in 4.6.2. Overall, these electrodes performed satisfactorily and did not suffer any material failures as was seen in Chapter 3. On a cycle level, the voltage profile showed reasonable agreement with literature. Individual analysis of each electrode or substrate is difficult as the author considers that the suitability of each electrode to its $C$ rate may have had a greater influence on the results obtained than the controlled variables.

On this basis, it is not possible to determine if the surface modification of the substrate had a discernible effect on the performance of the electrodes.

It is deemed that manufacture of a high temperature sintered iron electrode is possible using the above-described method. Reduced heating times compared to those reported here may be possible using more powerful equipment. This research on manufacture of sintered iron electrodes using rapid heating methods such as the HDS warrants further investigation.

\subsection{Blowing Agent Electrode for Further Investigation}

Attempts were also made to produce an electrode with an increased surface area through the use of blowing agents mixed directly in to the ink prior to heating. This is desirable as it could produce performance improvements in the coating and is a logical progression required to produce complex multi-layered coatings [105], [107].

Blowing agents can be used to increase porosity (and thus surface area) in a coating due to their ability to undergo significant volume expansion under heating. Expancel 950 DU 80 (Boud Minerals) was identified for this work due to its relatively high thermal 
decomposition temperature of $133-143{ }^{\circ} \mathrm{C}$. It was expected that this would be compatible with the temperature at which the iron inks solidify.

A poor match between the working range of the blowing agent and solidification temperature of the ink would result in minimal effect on the surface area. If the operating temperature of the blowing agent was too low, it would expand and then the pores would collapse again before the ink solidifies. If the blowing agent did not activate until after the ink had solidified, it would not be able to cause the same increase in surface area.

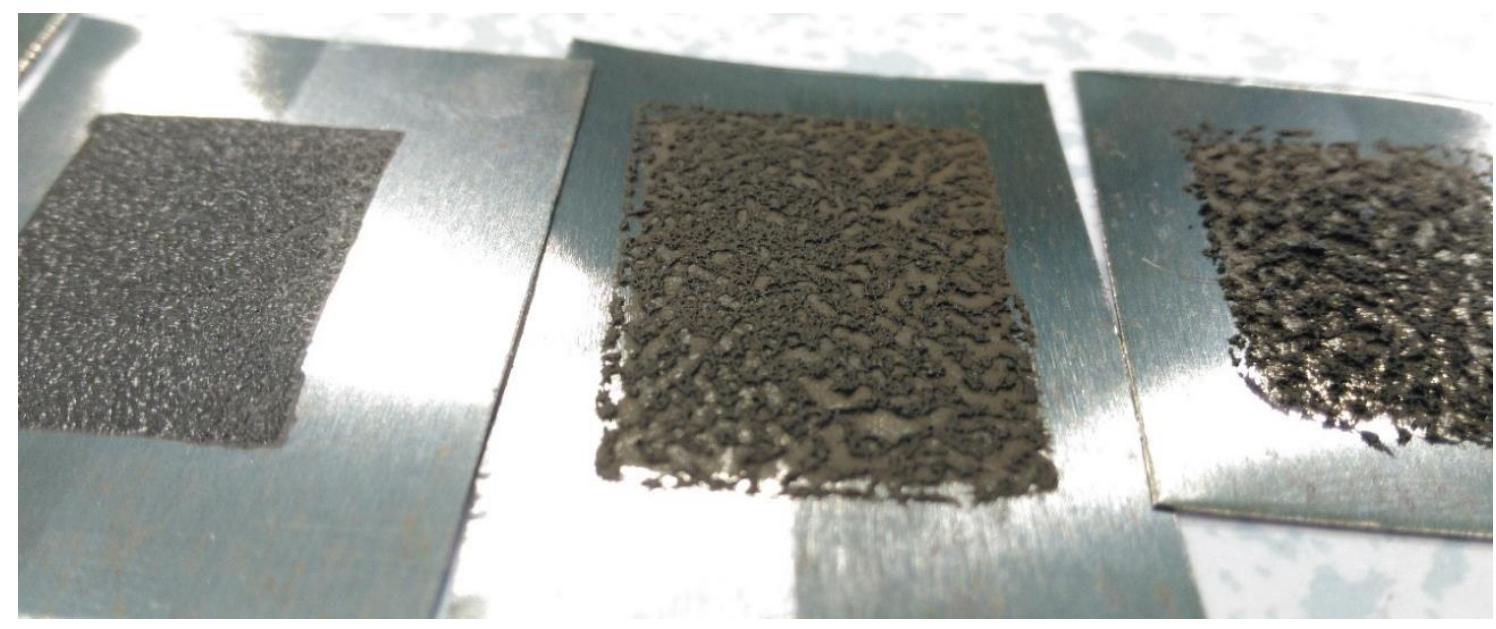

\section{Figure 55 Various volume fractions of Expancel blowing agent added to iron ink}

A range of coatings were produced with varying additions of Expancel blowing agent mixed by hand into the ink before coating. These were heated on a hotplate to solidify and observations were made on the amount of blowing agent used (Figure 55).

Based on these observations, additional ink was produced using $0.61 \mathrm{wt} \%$ of Expancel 950 DU 80 (Boud) in Iron ink C2150413D1 (Gwent Electronic Materials Ltd.). This was coated and sintered in the manner described previously (4.5.7) on a 'ground' substrate. 


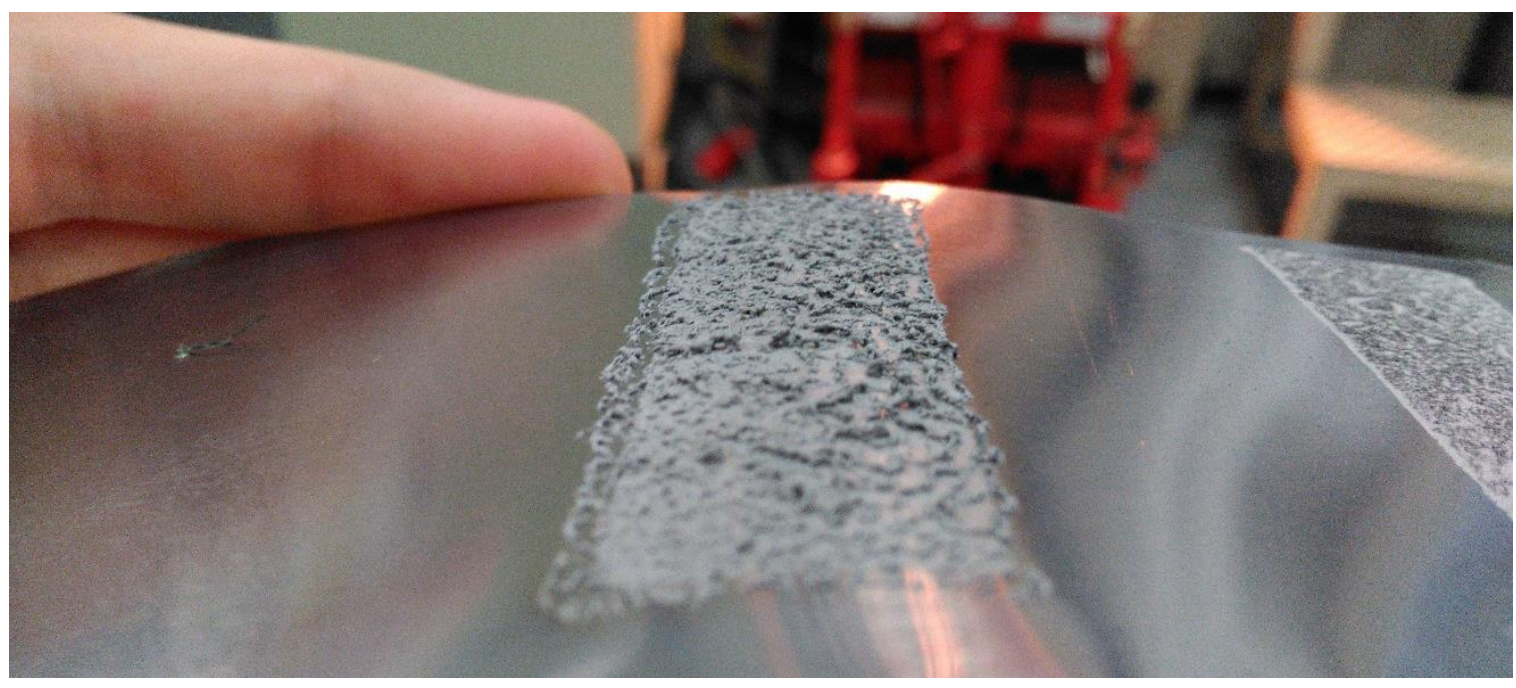

Figure 56 Sintered iron using Expancel blowing agent

This Figure 56 sample enabled additional investigation as described in 5.5.2. These electrodes were not electrochemically cycled.

\subsection{Further Work}

Producing additional electrodes on each substrate would have allowed for a larger sample size and thus a greater ability to determine the validity of results obtained. Extensions to this work should greatly increase the number of samples produced.

Incorporation of inhibitors directly in to the electrode could improve the efficiency of the cell. However, it is noted by Yang et al that that evaporation can be an issue with some well performing additives [19].

Further investigation could also be performed with electrolyte additives in sintered electrodes, such as those used in other work by this researcher (Chapter 3 and 5.5).

Sintered electrodes could also be tested with a novel method such as the Scanning Vibrating Electrode Technique (5.5.2). 


\section{Chapter 5: A Scanning Vibrating Electrode Technique for the Study of Hydrogen Evolving from an Iron Electrode Battery}

\subsection{Introduction}

Research presented in Chapter 3 and Chapter 4 has centered around efforts to manufacture iron electrodes at low and high temperatures, with the focus on rapid manufacture to reduce the inherent cost. However, a major drawback of iron electrodes are the low charging efficiencies [22], [69], [81] caused by a parasitic hydrogen evolution reaction. More advanced analytical techniques should improve the understanding of these reactions and assist in the development of inhibitors to prevent or slow detrimental reactions.

To further understand the parasitic hydrogen evolution reaction, an in-situ Scanning Vibrating Electrode Technique (SVET) is employed for the first time to study the influence of a range of inhibitors on the rate of hydrogen evolving from a charging iron electrode when fully immersed in $\mathrm{KOH}$ electrolyte solution $(30 \% \mathrm{w} / \mathrm{v})$.

Here, the researcher has confirmed the accuracy of SVET-derived hydrogen evolution data through comparison to results obtained using a volumetric gas collection technique and cyclic voltammetry experiments. This is required as experiments using SVET have not previously been carried out in strong alkaline conditions. Therefore, verification experimentation will be performed against traditional techniques, as suggested by Professor Mabbett (Swansea University). Electrolyte additives will be used to assess the qualitative performance using both techniques.

SVET offers an advantage over traditional techniques, as the use of a scanning probe means researchers can produce surface maps of the intensity and location of electrochemical activity with respect to time [155], [156], [203]-[206]. This allows for investigations focusing on the level of heterogeneity of electrode current density during charging. Non-planar samples can be examined through use of a 3D height scan to map the surface topography [207]-[209]. Once the accuracy of the technique has been confirmed, this researcher will examine electrodes made in previous work. 
Development of the SVET as an analytical technique should allow the assessment of the effect of various electrolyte additives to suppress the hydrogen evolution reaction. Here, three electrolyte inhibitors, namely sulfur, lithium hydroxide and thiourea were selected for their compatibility with the highly alkaline $\mathrm{KOH}$ electrolyte and previous use in hydrogen evolution research. Previous reports suggest that sulfur additions improve cycling-ability and formation of the iron electrode during charging by aiding reduction of trivalent iron [19], [75], [76], [120]. As discussed in 1.6.3, lithium hydroxide is commonly employed for various functions including improving cell capacity and cycling efficiency [18], [87], [119], [202]. It is proposed that these performance improvements are due to formation of a catalytic lithium-iron-oxide intermediate [118]. Further performance gains are possible through use of thiourea, which has been shown previously to raise the overvoltage of the hydrogen evolution reaction [121]-[123].

Developmental work is required for this powerful analytical technique to be used in this environment. Prior to investigation using the SVET, specialist sample holding equipment required designing and manufacturing. This was essential due to the strong alkaline conditions typically used in iron electrode batteries. Typical sample holders used for SVET studies would be unable to operate effectively in strong alkaline conditions.

\subsection{Method Development: Sample Holder}

Prior use of the Scanning Vibrating Electrode Technique is limited to studying ferrous corrosion and magnesium in saline conditions [156], [203], [210]. More recently, similar tests have been performed using Methanol as an electrolyte [211]. Studies in strong alkaline conditions have not been performed previously.

Here, the essential method development required to enable iron electrode research using the SVET is discussed. 


\subsubsection{Requirement}

\section{Electrolytes for iron electrodes}

Alkaline batteries are the main subject of research; therefore, it is important to understand the conditions in which these cells operate. Typically potassium hydroxide is used as an electrolyte at a concentration of $25-40 \% \mathrm{w} / \mathrm{v}[60]$.

Potassium hydroxide at these concentrations is both corrosive and toxic. It will gradually dissolve glass over a few days and will corrode aluminium, brass, bronze, some polymers and many adhesives. Polypropylene, polyethylene, Acetal/Delrin, Acrylic, PTFE, EPDM and PVC all offer excellent chemical resistance. Therefore, testing equipment must be designed using materials which are chemically resistant to potassium hydroxide.

\subsubsection{Preliminary Sample Holder Designs}

\section{Requirements}

For test equipment to be compatible with the conditions encountered in alkaline batteries, they must meet the following requirements:

- Chemical resistance at high $\mathrm{pH}$ to maintain structure and avoid contaminants leaching into test electrolyte

- Non-metallic and non-conducting

- Non-porous, can be kept clean

- Expose a 10 x $10 \mathrm{~mm}$ sample area in a repeatable manner

- Include a levelling stage which can be adjusted to within $10 \mu \mathrm{m}$ for SVET use

- Electrical connection to the sample for polarization studies

- Reusable (preferably)

Typically, electrochemistry samples are mounted in a non-conductive resin (such as Struers ${ }^{\circledR}$ DuroCit ${ }^{\circledR}$ Acrylic resin) for polishing. It is possible to add an electrical 
connection to the sample before mounting, which is essential for polarization work. The required test area is normally masked off with PTFE tape (3M 5490), cut to size using a scalpel. This method is usually employed when studying galvanized layers and other corrosion activity using the SVET and is very successful and repeatable.

However, it was discovered that the adhesive in PTFE tape was attacked rapidly by the potassium hydroxide resulting in delamination from the sample surface. This had to be avoided for the test to be successful.

Several methods for holding flat metallic samples were trialed.

\section{Loctite® Double Bubble}

Sample masking was attempted using Loctite ${ }^{\circledR}$ Double Bubble Epoxy to mask a 10 x 10 $\mathrm{mm}$ area on a mounted sample (Figure 57). However, this adhesive tended to delaminate from the surface during more vigorous hydrogen evolution, gradually increasing the sample area, making it unsuitable for use (Figure 58).

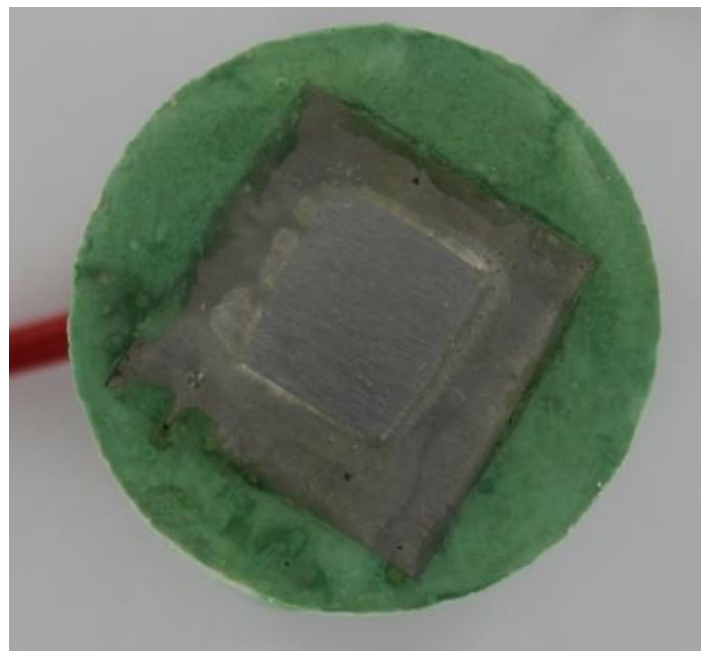

Figure 57 Sample masked using Loctite Double Bubble Adhesive

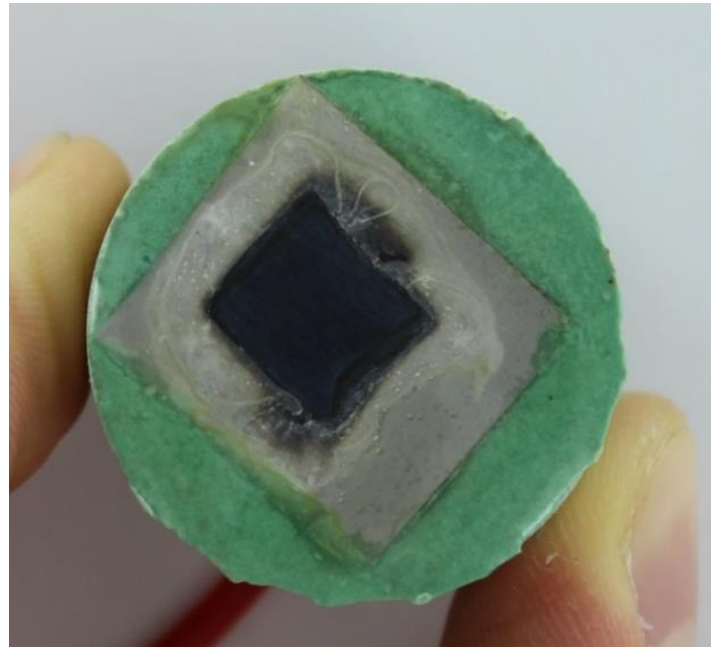

Figure 58 Adhesive delamination as a result of hydrogen evolution

Lack of peel strength and chemical resistance meant that this glue was unsuitable. An alternative glue with increased peel strength was sourced. 


\section{Alpha SAS 520}

Tests were performed using samples which had been prepared using Alpha SAS 520 Epoxy, which claims exceptionally high peel strength. This adhesive had also performed well against potassium hydroxide chemical resistance tests. However, a higher viscosity and longer working time than Loctite ${ }^{\circledR}$ Double Bubble makes it more difficult to accurately mask off $10 \mathrm{~mm}^{2}$ (Figure 59).

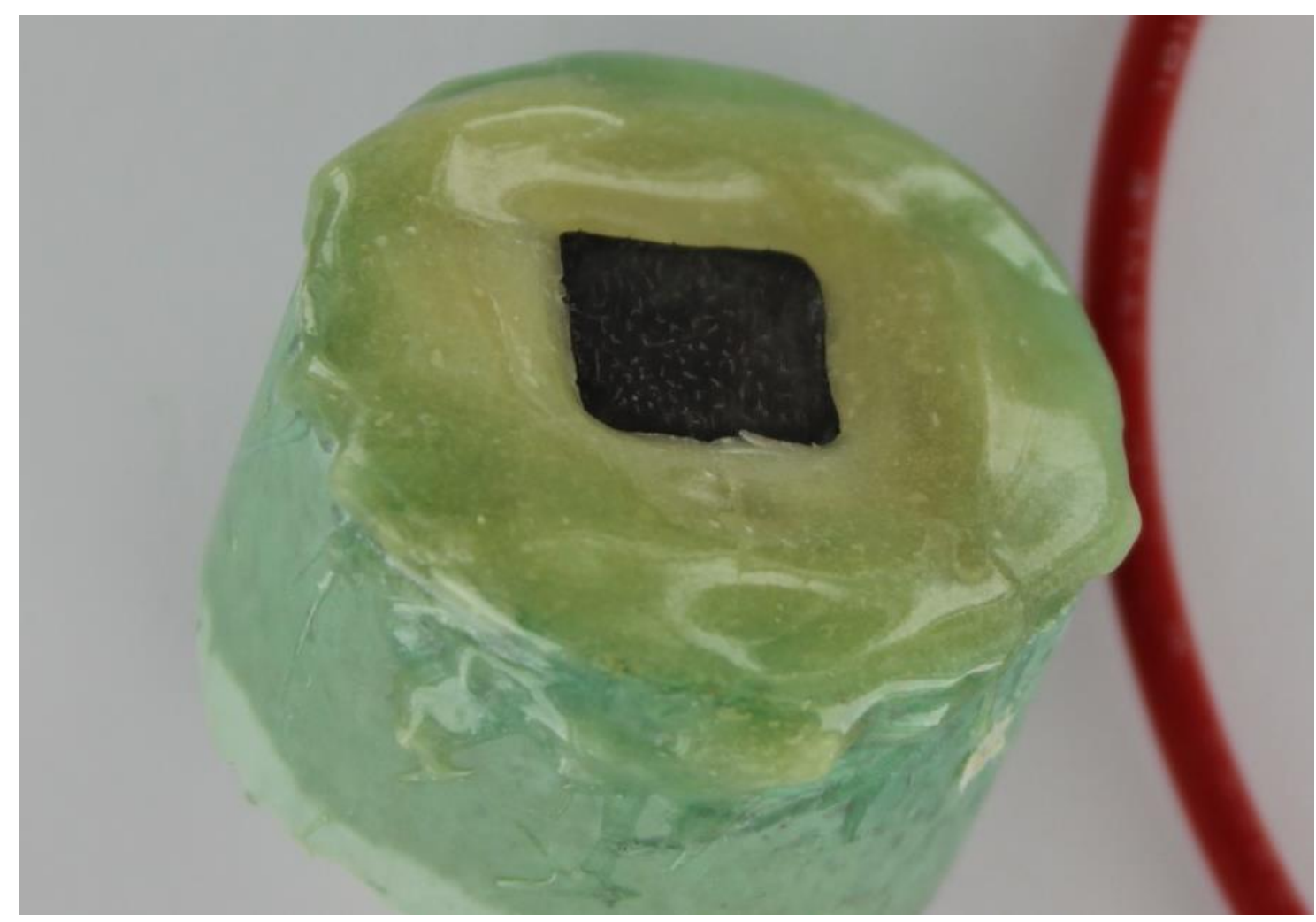

Figure 59 Sample mounted using Alpha SAS 520 adhesive

Despite the higher peel resistance, a small amount of de-bonding still occurred.

\section{RS Epoxy Putty/Paste}

Masking samples using epoxy putty was challenging; it was very difficult to mask off a particular area. Poor edge retention around the sample being masked off resulted in testing using this material being discontinued (Figure 60). 


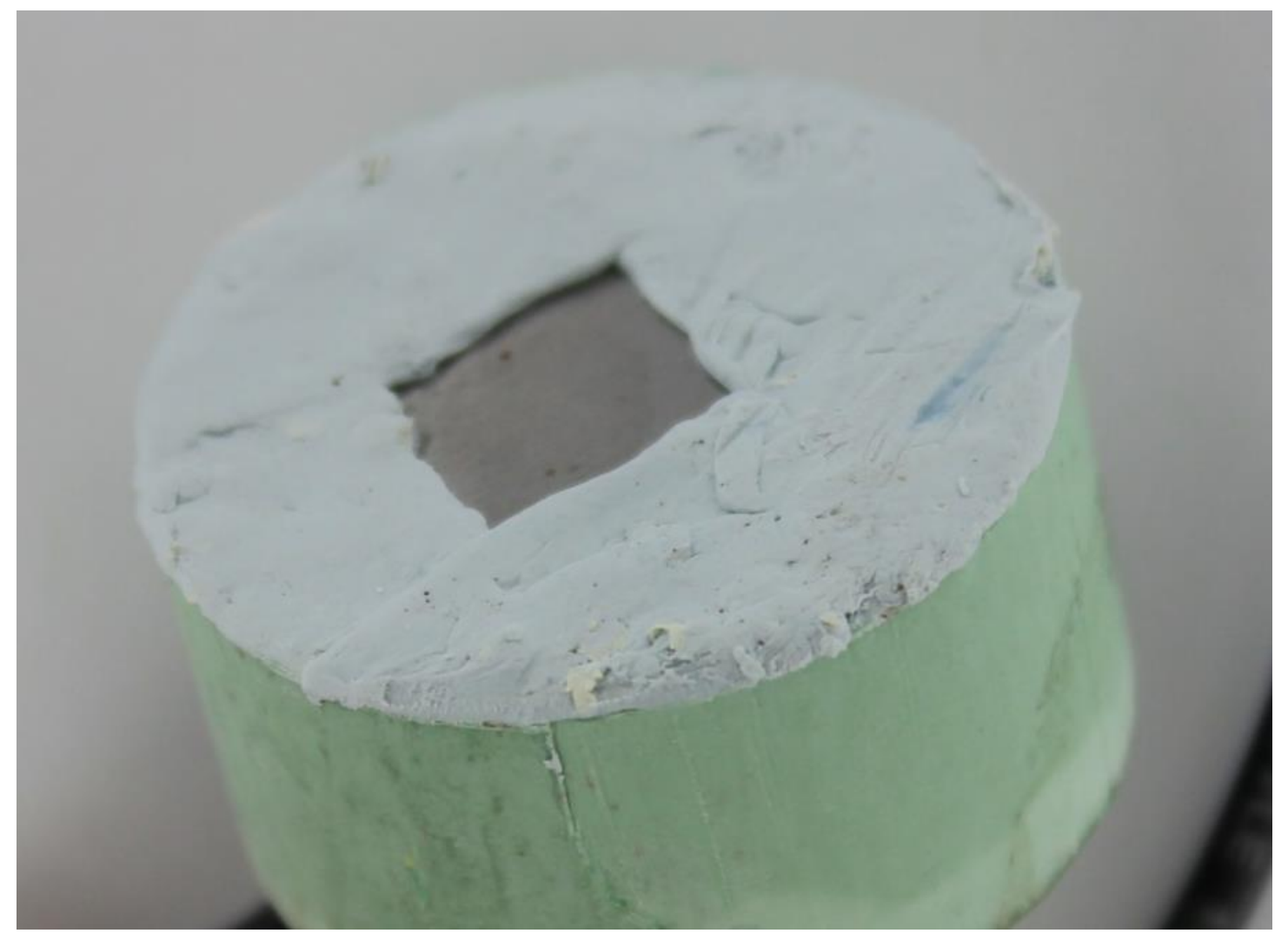

Figure 60 Sample area masked off using RS Putty Epoxy

\subsubsection{Initial Concepts for Reusable Sample Holders}

Development of a reusable sample holder allowed for a fresh sample to be loaded in to the sample holder, immediately prior to an experiment. This reduced variation in sample preparation and limited time between any polishing and testing of the materials.

Various reusable sample holder systems were developed.

\section{Laser cut sample holder}

An Epilog Mini 40 Watt $\mathrm{CO}_{2}$ laser engraver/cutter was utilized to rapidly produce different iterations of sample holders cut from sheet Acrylic. This equipment was suggested by fellow researcher, Dr. C Glover (Swansea University). It was discovered that using a shaded CAD drawing (Figure 61), this equipment could be used to accurately cut a chamfered profile into the Acrylic (Figure 62). This allowed a convenient profile for a sample holder which would allow easy access to the SVET probe. 


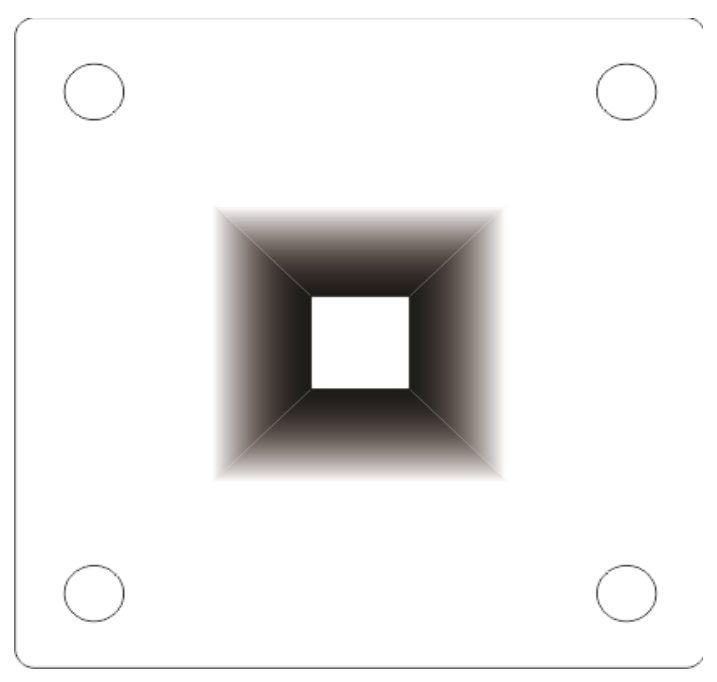

Figure 61 Top profile of sample holder showing shaded chamfer cut

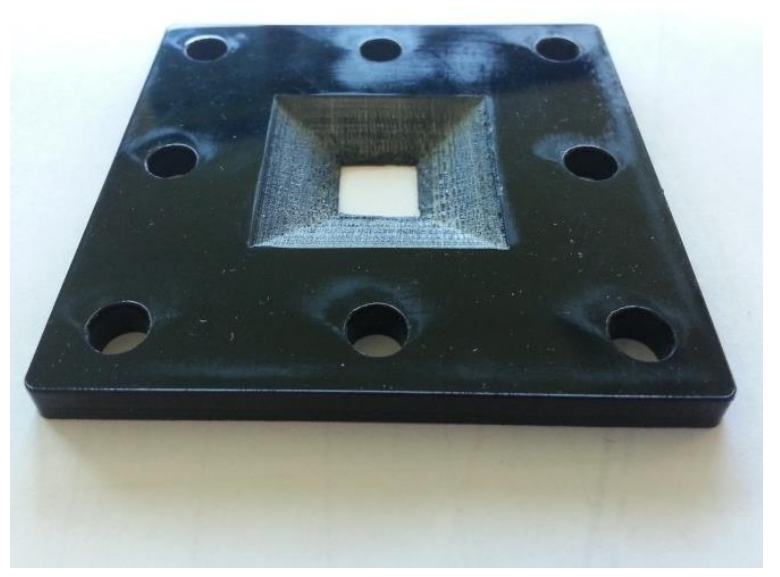

Figure 62 Chamfered profile cut using a laser cutter

A layered structure was designed such that a sample could be placed under the chamfered area and compressed with nylon nuts and bolts. Sealing was attempted using $3 \mathrm{~mm}$ thick polypropylene foam, it was not possible to achieve reliable sealing in this material. A $0.5 \mathrm{~mm}$ EPDM sheet was also tested but stiffness of the acrylic limited the ability to seal.

Various modifications were made on this design with the intention of improving stiffness of the sample holder, these were unsuccessful. Alternative materials were considered; however, the required dimensions could not be achieved due to heat build-up during cutting.

\section{Delrin sample holder prototype}

In the absence of CNC machining equipment, a lathe was used to manufacture a screw top sample holder from an Acetal variant Delrin ${ }^{\circledR}$, manufactured by Dupont ${ }^{\circledR}$. This material was chosen due to a high stiffness and machinability combined with excellent chemical resistance.

In an attempt to improve the sealing of the sample holder, the lid was designed to screw on to the base. It was thought that this closure mechanism would result in the higher compressive force on the sample and gasket and reduce leaking. $0.5 \mathrm{~mm}$ EPDM rubber 
was used to provide a seal on top of the sample, but was later replaced with $1 \mathrm{~mm}$ EPDM due to the increased compliance and superior sealing.

As with the previous iteration of the acrylic sample holder, the chamfered opening for access to the $10 \mathrm{~mm}^{2}$ sample for the SVET probe was cut using a laser cutter (Figure 63).

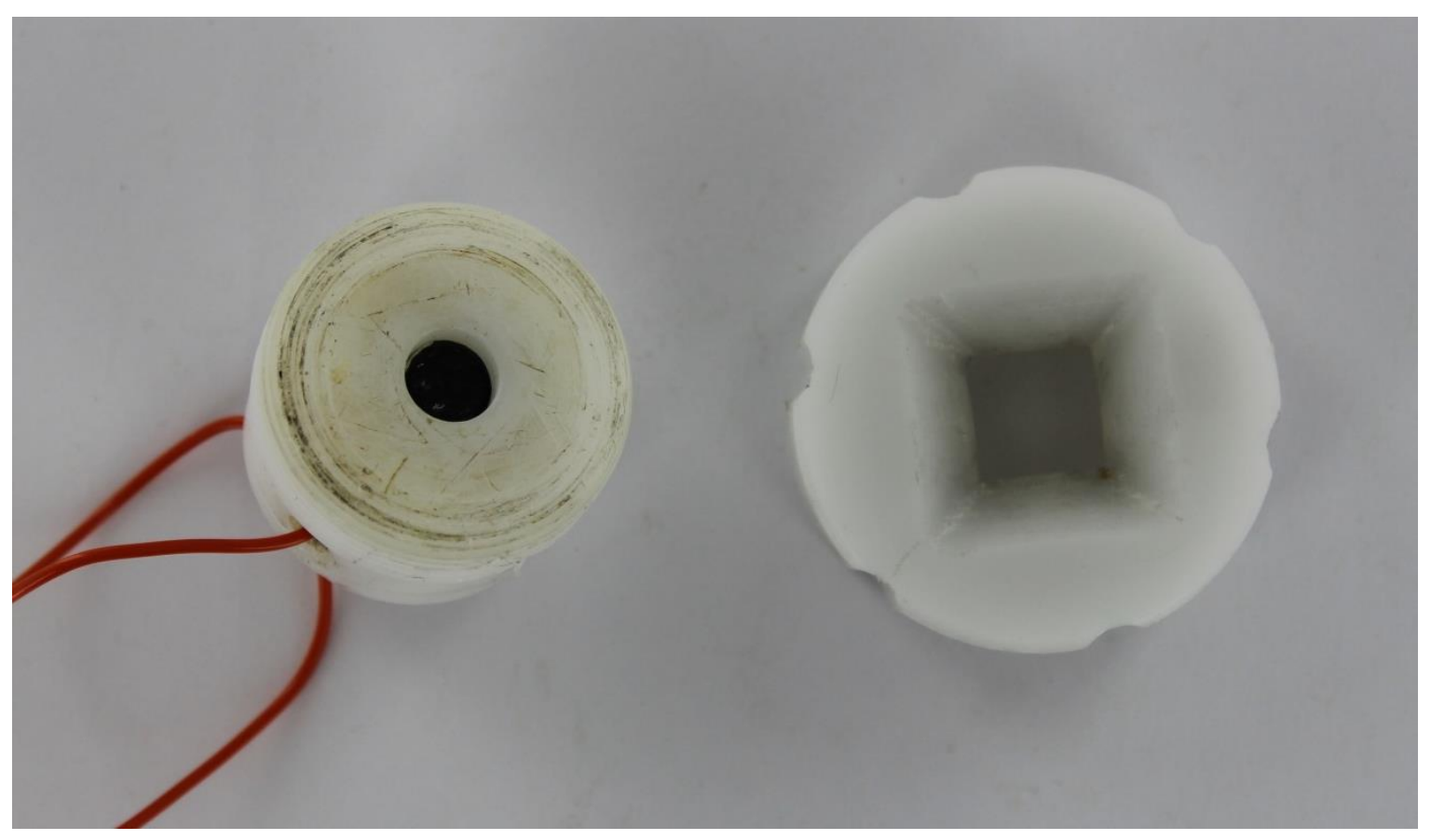

Figure 63 Acetal sample holder

In principle, this design was much more successful than previous designs. Equipment availability required for further designs to be manufactured by an external contractor.

Several areas for improvement were recorded:

- Occasional leakage via the gasket (located at underside of lid)

- Difficulty tightening sample holder to required torque

- Cable entry point was weak and prone to failure

\section{Delrin CNC Sample holder design}

Computer Aided Design software was used by this researcher to design an improved sample holder (Figure 64 and Figure 65). This was to be Computer Numerical Control (CNC) manufactured with higher tolerances than the previous design. The lid and base 
were altered so that they could be tightened using a vice and spanner. Previous design iterations had suffered with poor stiffness in the sample holder lid, at the edge of the chamfer. When tightening the sample holder this area would flex, resulting in a poor seal. For this design, a small step of $0.1 \mathrm{~mm}$ was added, in order to increase the stiffness around the periphery of the $10 \mathrm{~mm}^{2}$ sample. The cable entry point was changed so that it could be sealed with chemically resistant Alpha SAS 520 two-part epoxy. $1 \mathrm{~mm}$ EPDM rubber was used as a gasket material (Figure 66 and Figure 67).

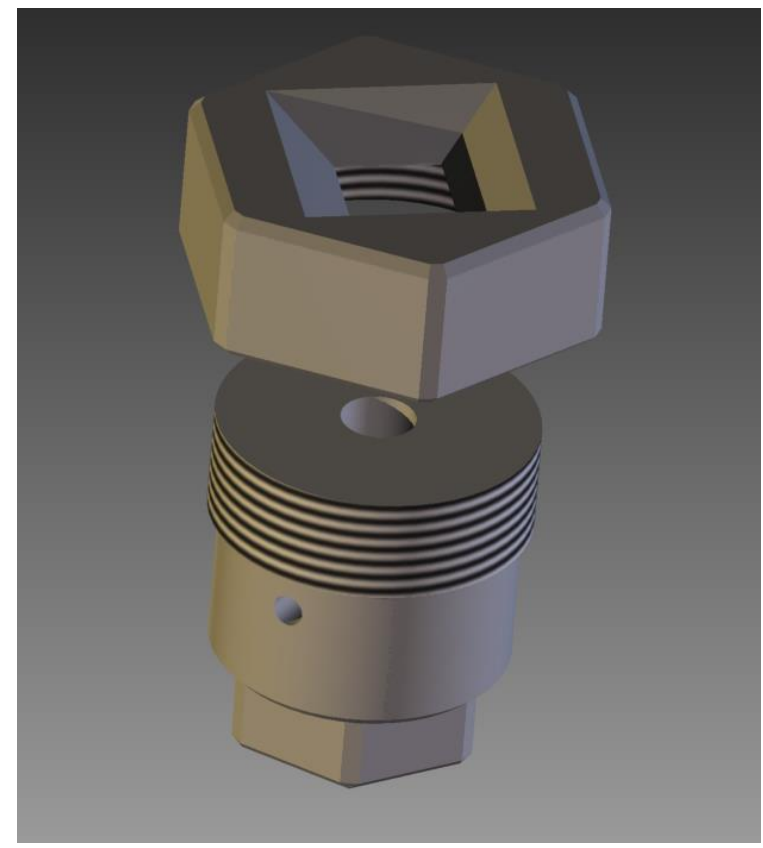

Figure 64 Rendered image of the designed sample holder

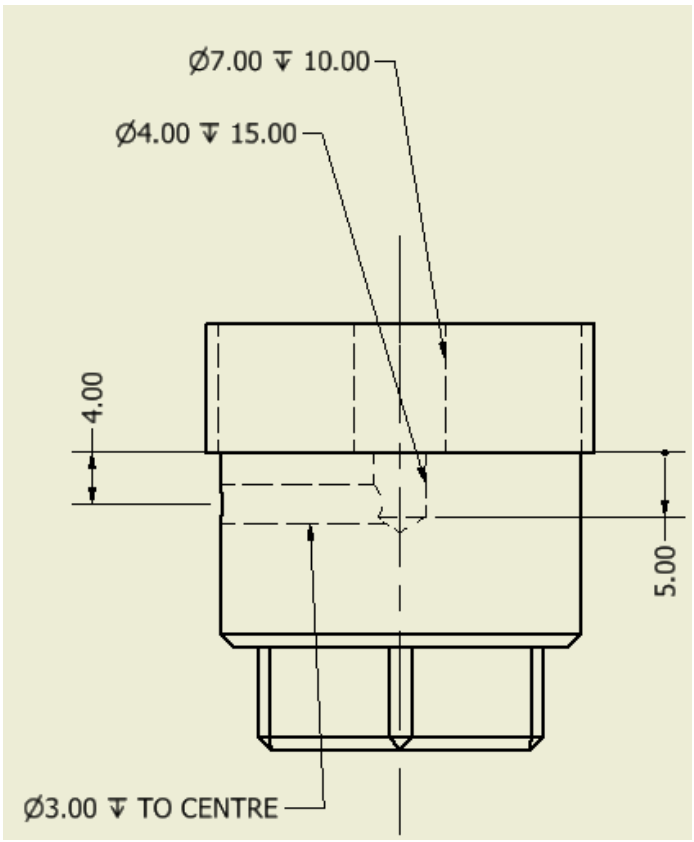

Figure 65 Cross section drawing of the base of the sample holder 


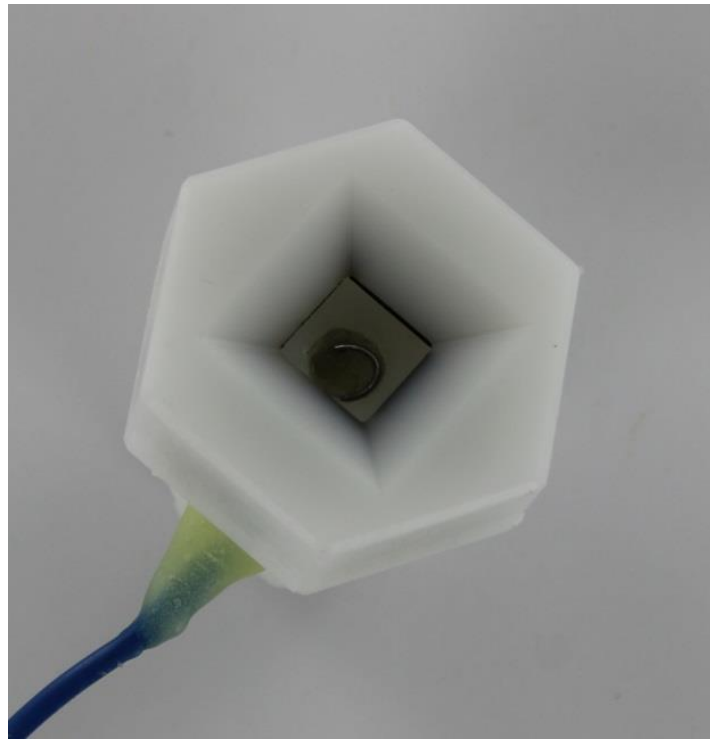

Figure 66 Assembled sample holder

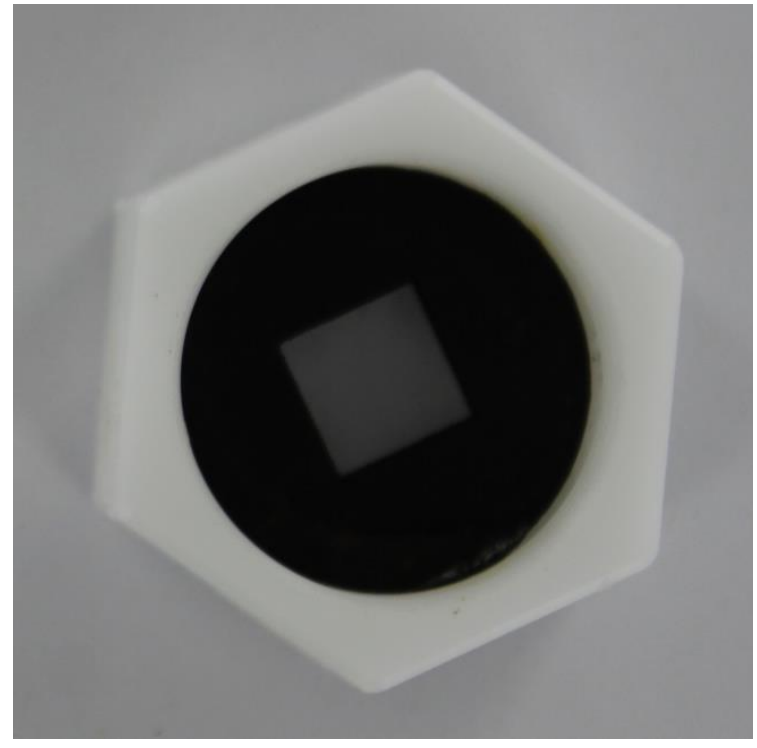

Figure 67 EPDM gasket in underside of sample holder lid

The new sample holder performed without leaking with the addition of PTFE sealing tape (RS Components) to seal the threads.

This design satisfied the design requirements. Samples can be polished and cleaned immediately prior to testing and quickly loaded and unloaded from the sample holder without degradation to the sample holder.

\subsubsection{Machined Sample Holder and Accessories}

\section{SVET Tank}

Dr. J. Searle (Swansea University) originally designed large Perspex tanks to contain the Electrolyte for use during SVET experimentation. These were manufactured from glued sheets to form an open topped tank. There were concerns that if this equipment was used for this researcher's experimentation with concentrated potassium hydroxide, the tank may leak or rupture. It was suggested by Dr. Searle that this researcher design and manufacture a tank made from one piece, thus not requiring adhesive. Threaded holes were also included for a levelling stage to attach to (Figure 68). 


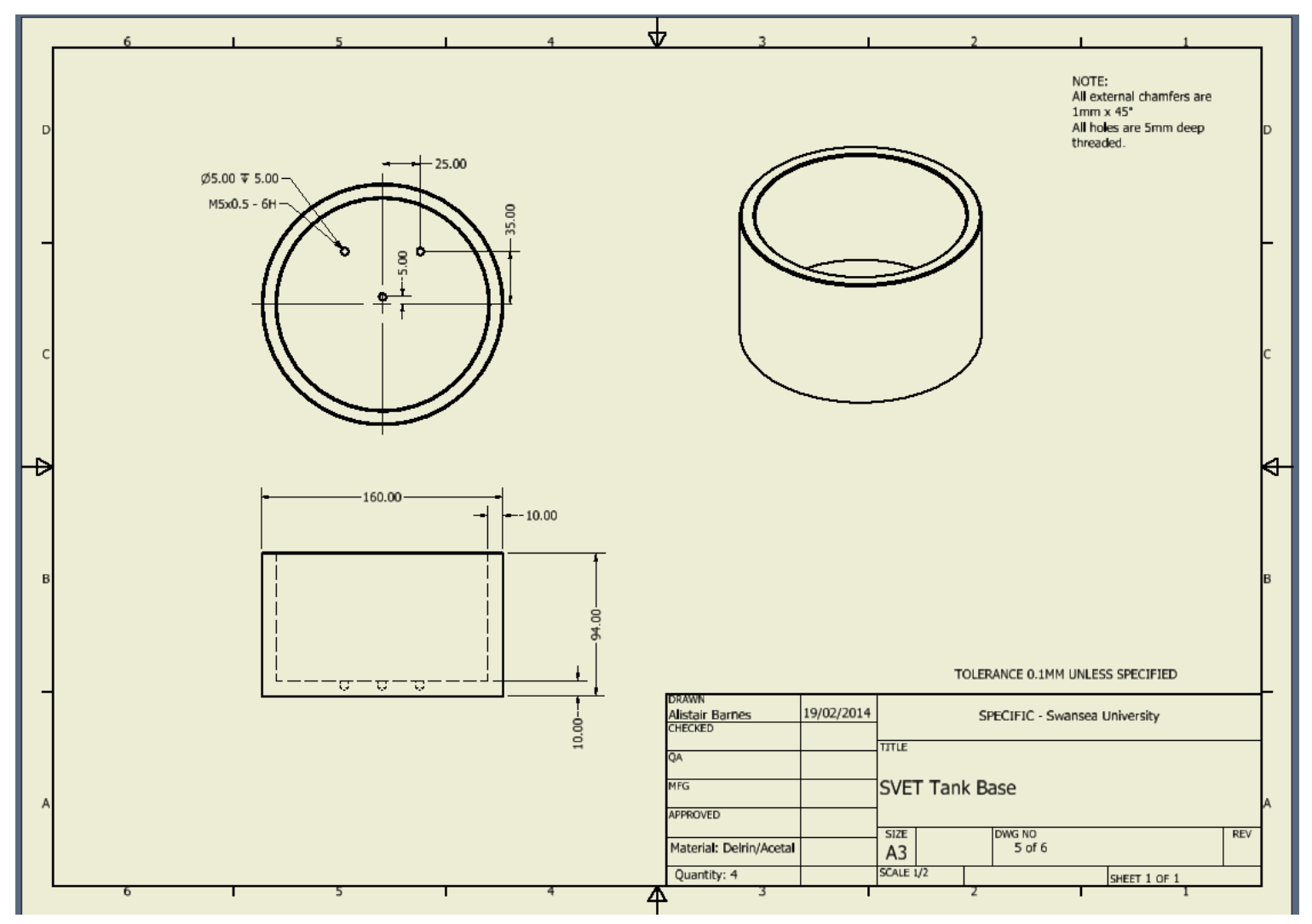

Figure 68 Engineering drawing of SVET tank

\section{Levelling stage}

When scanning planar samples, the SVET is typically operated without a 3D height scan. In order to attain a constant height between the sample and the SVET probe, the sample must be levelled with respect to the SVET axis. This is achieved using a two-part stage which is separated by thumbscrews on each corner (Figure 69). These screws can be wound in or out to adjust the height of each corner of the sample. 


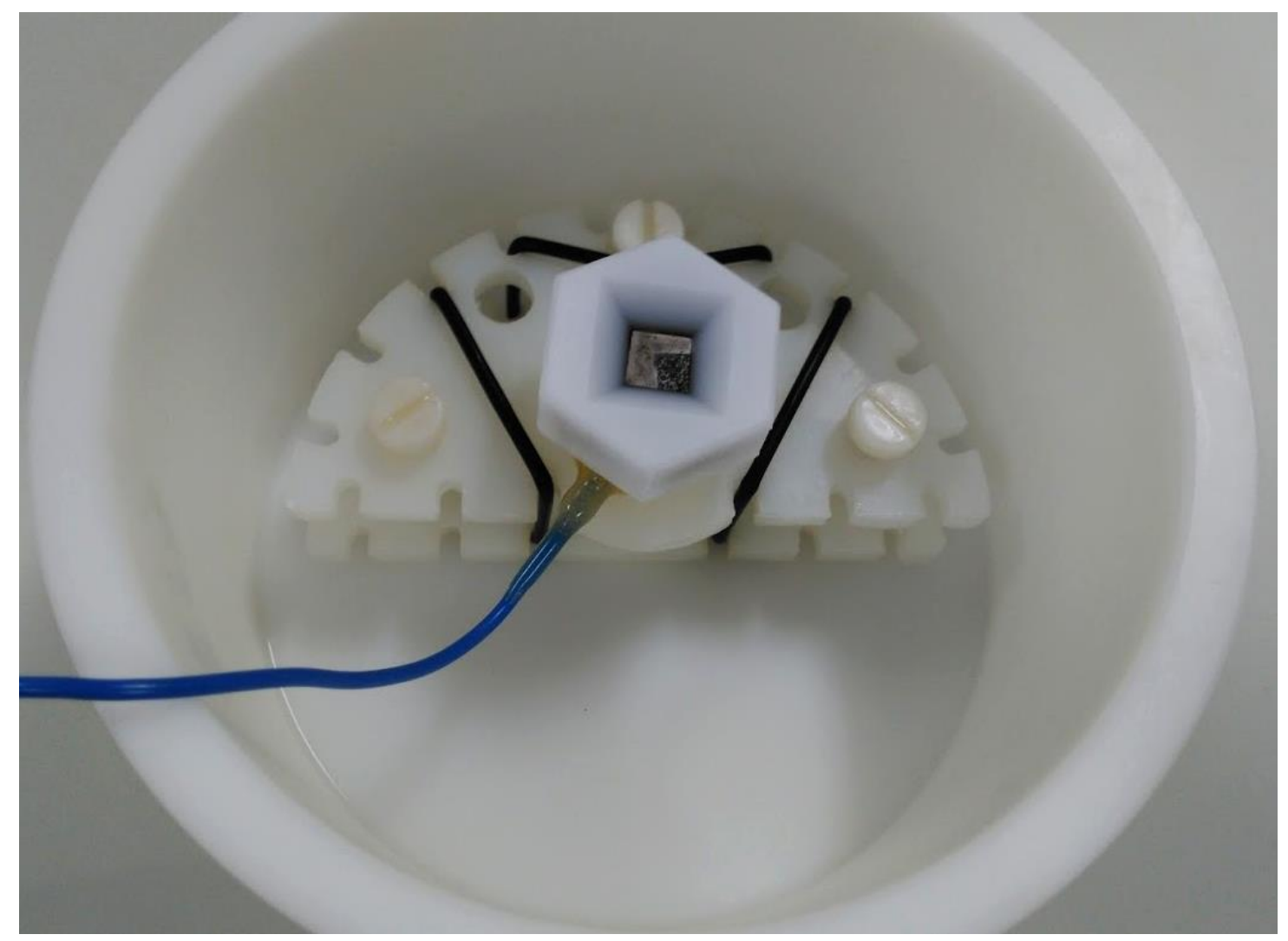

Figure 69 SVET tank with adjustable levelling stage and sample holder

Again, the laser cutter was utilized in order to provide a rapid method of manufacturing this item using chemically resistant polypropylene. O-rings were used to hold the sample holder together and prevent movement during use. Nylon screws were used to adjust the height of the stage after confirming chemical compatibility.

\section{Electrochemistry cell holder}

Various tests were to be performed using cyclic voltammetry. This required a cylindrical sample holder lid and careful control of the distance between the working, reference and counter electrodes.

An Acetal lid was manufactured to hold the electrodes in their required position. Grub screws were used to hold each item in place. A barb fitting for a gas line was included as a nitrogen line might be required for testing. An Acetal tank was also produced (not pictured). 


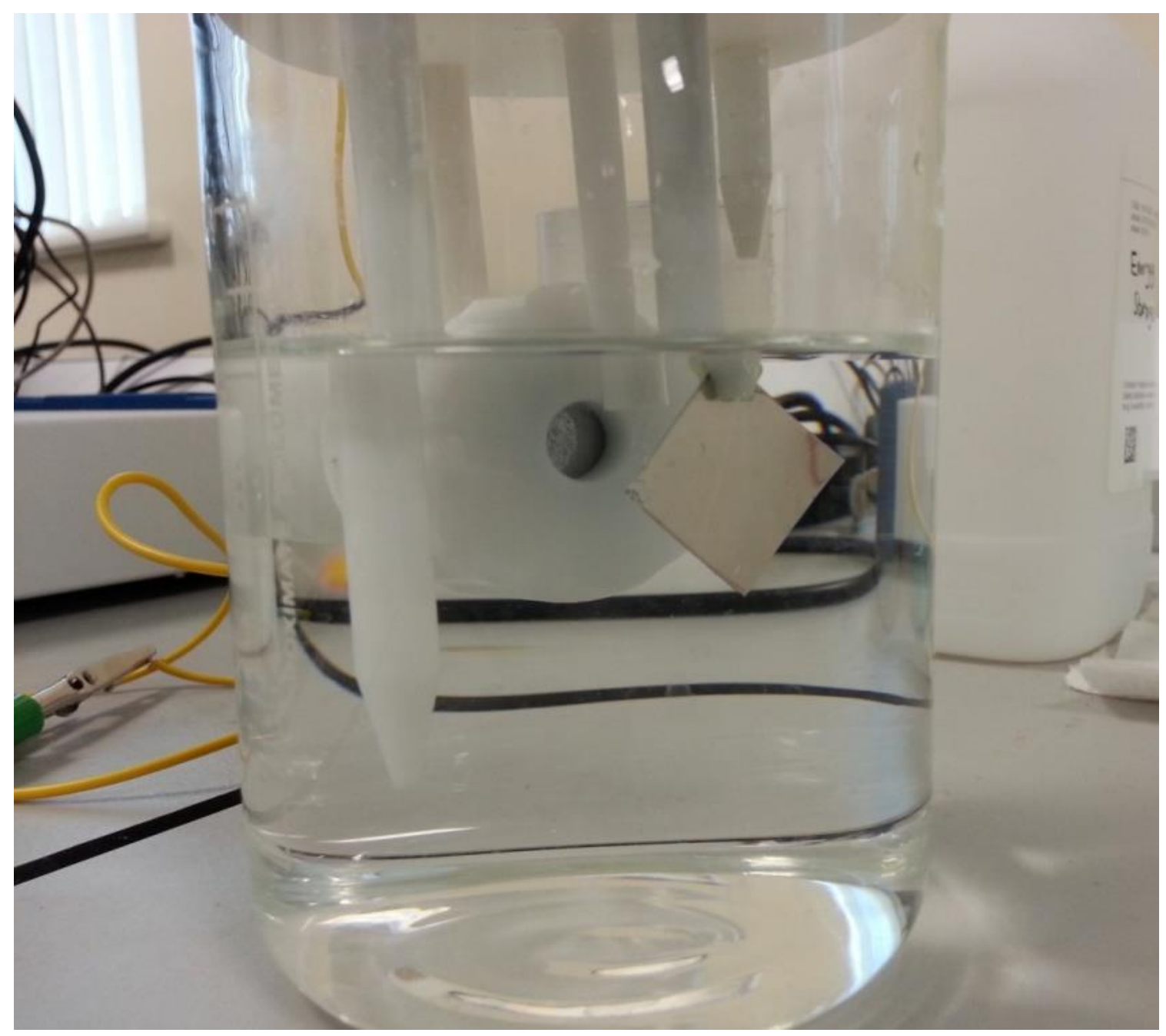

Figure 70 DC electrochemistry cell holder

\section{Outcomes}

The design and manufacture of the sample holder and associated items (Figure 71) allow for the experimentation discussed in Section 5.1 to be completed. The SVET tank combined with the stage and sample holder can be used during SVET tests, but also in a volumetric test where gas is evolved and captured using a burette. Cyclic voltammetry can be performed using the vertical electrochemistry sample holder. Initial testing with this equipment showed that it met the 5.2.1 requirements and did not leak during operation, allowing to be used for further experimentation. 


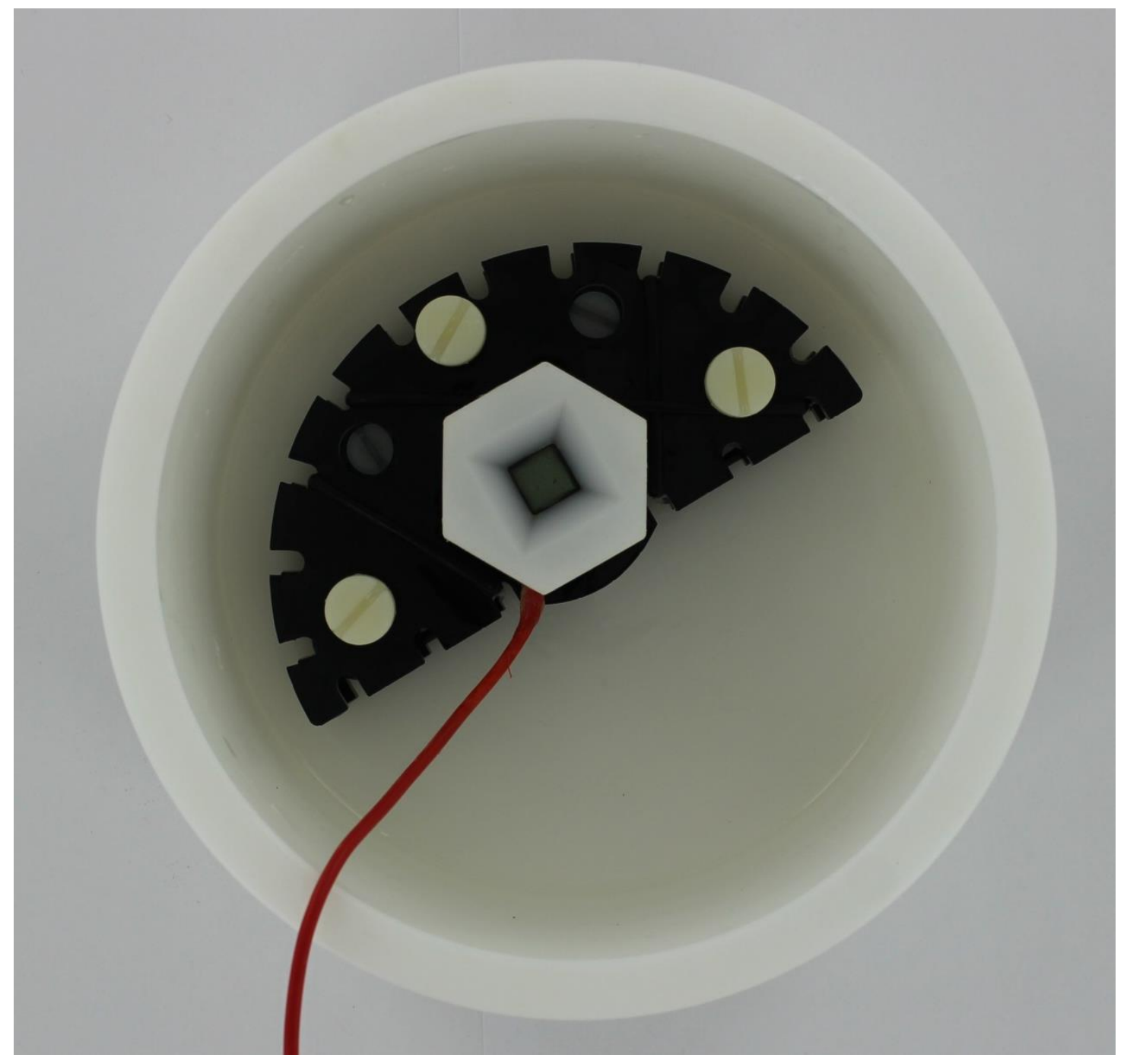

Figure 71 Completed sample holder and stage in Delrin ${ }^{\circledR}$ electrolyte tank

\subsection{Experimental Details}

Following the preliminary work in designing sample holders, the primary experimental work in this chapter is presented.

\subsubsection{Materials}

Iron (99.5\% purity $\mathrm{Fe}$ ) discs, of $1 \mathrm{~mm}$ thickness, were supplied by Goodfellow Metals Ltd. All other chemicals were supplied by Aldrich Chemicals. Iron samples were polished to a mirror finish as described in 2.2.1. 
Iron was selected as it is the active material used in previous experimental chapters. In previous works this researcher used a steel substrate provided by the project's sponsor, however it was no longer appropriate to use this as it does not sufficiently simulate the iron active material. Polishing was performed to ensure a repeatable and consistent electrodes for initial electrochemistry work. Later, scanning electrochemistry is conducted on bespoke electrodes manufactured by this researcher.

\subsubsection{Methods}

Cyclic voltammograms were recorded with guidance and practical assistance from Dr. Raman Subramanian (Swansea University) using a Solartron 1287 electrochemical interface. Scans were carried out between $-1.0 \mathrm{~V}$ and $-1.4 \mathrm{~V}$ vs. $\mathrm{Hg} / \mathrm{HgO}$ at a scan rate of $100 \mathrm{mVs}-1$. Polarization experiments were limited to these regions to avoid formation of iron oxides and hydroxides. In each case the iron electrode area was $0.95 \mathrm{~cm}^{2}$ and the counter electrode was a platinum mesh. Each experiment was carried out in $400 \mathrm{ml}$ of $\mathrm{KOH}(30 \% \mathrm{w} / \mathrm{v}$ solution) where additions of the relevant inhibitor were made at the following concentrations: sulfur - $0.1 \mathrm{M}$ (saturated), thiourea - $0.1 \mathrm{M}$ and lithium hydroxide $-0.19 \mathrm{M}$ (in line with commercial cells [26]). The DC electrochemistry sample holder designed in Section 5.2.4 was used for testing.

SVET measurements were carried out using the equipment described in 2.2. This researcher acknowledges repeated assistance from fellow researcher J. Marinaccio in the manufacture of SVET probes following breakages and occasional practical assistance in experimentation. To conduct experiments, $10 \times 10 \mathrm{~mm}$ of iron was held in a sample holder (5.2.4) and completely immersed in $700 \mathrm{ml}$ of $\mathrm{KOH}(30 \% \mathrm{w} / \mathrm{v}$ solution) electrolyte. Experimentation was completed in both the presence and absence of, inhibitors. The electrolyte bath was left unstirred and in contact with room air at a nominal temperature of $20{ }^{\circ} \mathrm{C}$. The sample was polarized to a value of $-1.4 \mathrm{~V}$ vs. $\mathrm{Hg} / \mathrm{HgO}$, as determined by preliminary cyclic voltammetry experiments as the optimum potential for the hydrogen evolution reaction. SVET scans were carried out immediately following the start of polarization and at $15 \mathrm{~min}$ intervals thereafter for $15 \mathrm{~h}$ periods. 
Volumetric hydrogen evolution measurements were carried out by the method discussed in 2.3 employing an arrangement consisting of an upturned, electrolyte filled burette and glass funnel to collect hydrogen gas bubbles evolved over the entirety of the exposed iron surface. Otherwise, gas collection experiment mirrored SVET experimentation.

\subsection{Results and Discussion}

\subsubsection{Cyclic Voltammetry}

A series of cyclic voltammograms are given in Figure 72, showing the region of interest where hydrogen evolution occurs. In the absence of inhibitor, the peak at $-1.4 \mathrm{~V}$ vs. $\mathrm{Hg} / \mathrm{HgO}$ reaches a maximum current density of $-0.0176 \mathrm{Acm}^{-2}$. This current density peak is decreased incrementally in the presence of inhibitors such that a ranking order is established where: lithium hydroxide $<$ sulfur $<$ thiourea. This shows that the addition of an inhibitor will reduce the amount of hydrogen evolved at this potential. In the case of thiourea, this is a reduction in current density of $80.1 \%$. All further experiments were carried out potentiostatically at an optimum potential of $-1.4 \mathrm{~V}$ vs. $\mathrm{Hg} / \mathrm{HgO}$ to enable a reliable comparative study of each of the inhibitors without causing excessive perturbation by gas bubbles against the SVET probe. 


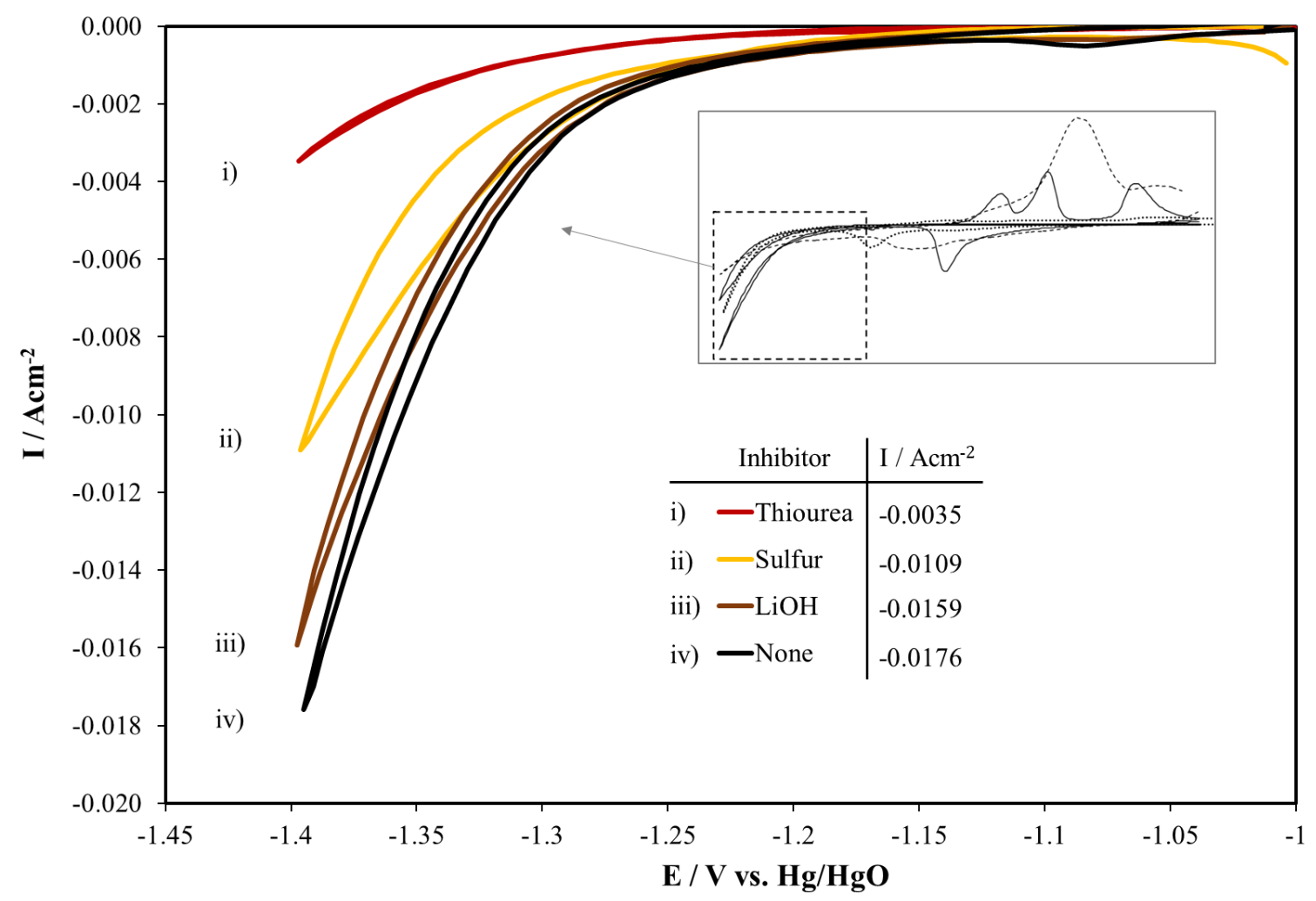

Figure 72 Typical cyclic voltammograms for a solid iron electrode in $30 \% \mathrm{w} / \mathrm{v}$ KOH electrolyte in the region in which hydrogen evolution occurs. With of i) thiourea, ii) sulfur, iii) lithium hydroxide and iv) no additions

\subsubsection{Scanning Vibrating Electrode Technique}

The SVET-derived current density surface maps shown in Figure 73 are typical of all those recorded in the study and show the current densities over the surface of an iron electrode when a) unpolarized and b) in charge conditions (polarized). When held at a potential of $-1.4 \mathrm{~V}$ vs. $\mathrm{Hg} / \mathrm{HgO}$, the mapped current density values are negative, illustrating the apparent cathodic nature of the surface reactions. The maps demonstrate the benefit of the SVET where the homogeneity of the current density distribution of the sample surface can be monitored. The surface activity detected by the SVET is extremely homogeneous, as would be expected. This researcher acknowledges contribution from Professors J. Sullivan and G. Williams (Swansea University) in the use of specialist software used for the presentation of Figure 73 and similar plots throughout this Chapter. 
a)

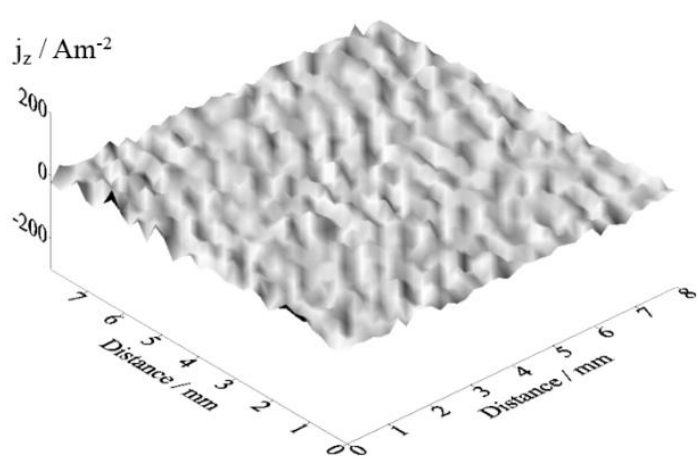

b)

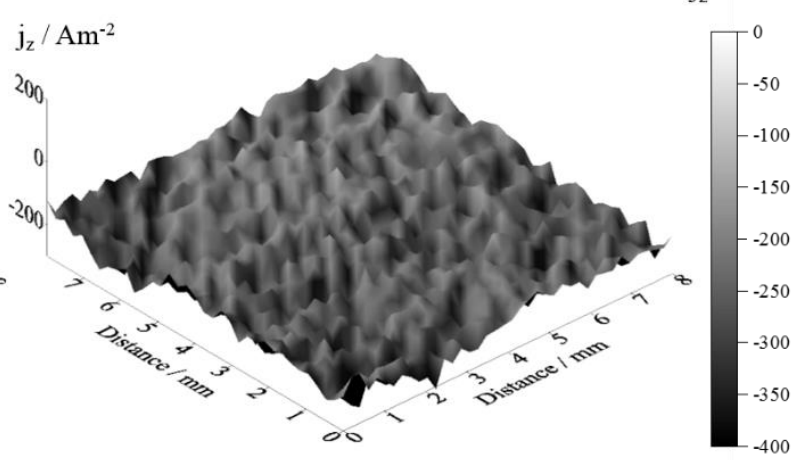

Figure 73 A typical SVET-derived current density surface map obtained by scanning an iron electrode surface when fully immersed in $30 \% \mathrm{w} / \mathrm{v} \mathrm{KOH}$ electrolyte, a) unpolarised and b), polarised to a potential of $-1.4 \mathrm{~V} \mathrm{vs}$. $\mathrm{Hg} / \mathrm{HgO}$

Figure 74a shows average SVET-derived values for gas evolved per $\mathrm{m}^{2}$ as a function of time for the various additives present in the electrolyte. Sulfur and Lithium Hydroxide show a reduction in the hydrogen evolution rate, by $40.1 \%$ and $25.9 \%$ respectively, when compared to the uninhibited case. A dramatic reduction of $92.0 \%$ is observed where thiourea additions have been made. The accuracy of gas-evolution rate, measured using the SVET, has been verified by comparison with data obtained using a traditional gas collection method - this is summarized in Figure $74 \mathrm{~b}$. Similar results are observed with each additive and both techniques are in agreement on a ranking order. These results are further confirmed with data from $\mathrm{CV}$ experiments where thiourea $>$ sulfur $>$ lithium hydroxide $>$ no additive. This shows that SVET is capable of accurate quantitative measurements with the advantage of spatial and temporal current density mapping.

SVET data obtained in the presence of sulfur additions shows a slight overestimation in comparison to gas collection data. Any electrochemical activity occurring at the surface will be detected by the SVET, not only the hydrogen evolution reaction. It is likely that this SVET overestimation is due to formation of sulfur containing species which do not result in gas evolution and therefore do not affect gas evolution rate [62], [87]. Equally, gas collection experimentation may underestimate efficiency losses which occur due to parasitic reactions, as they will only account for reactions which evolve gas. 
a)

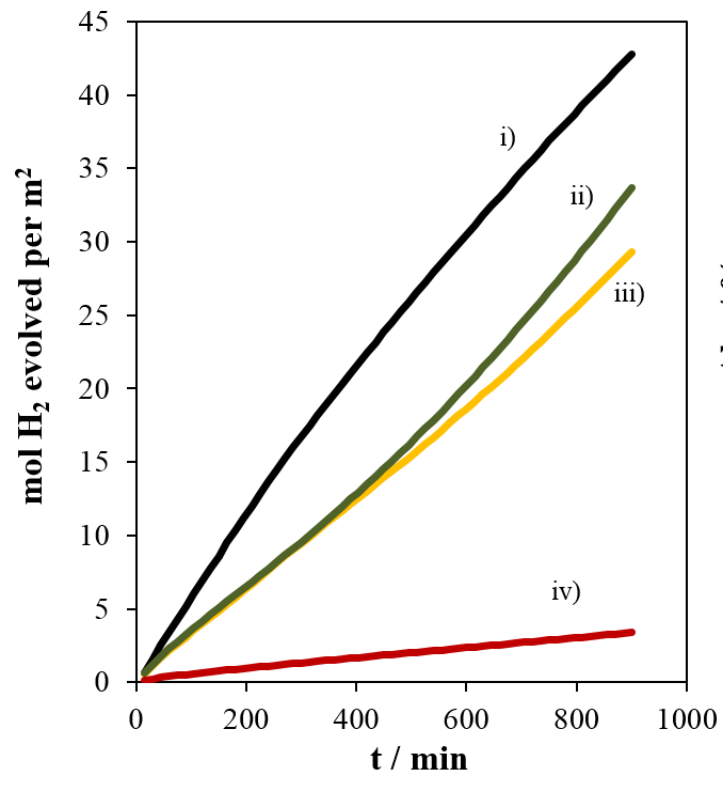

b)

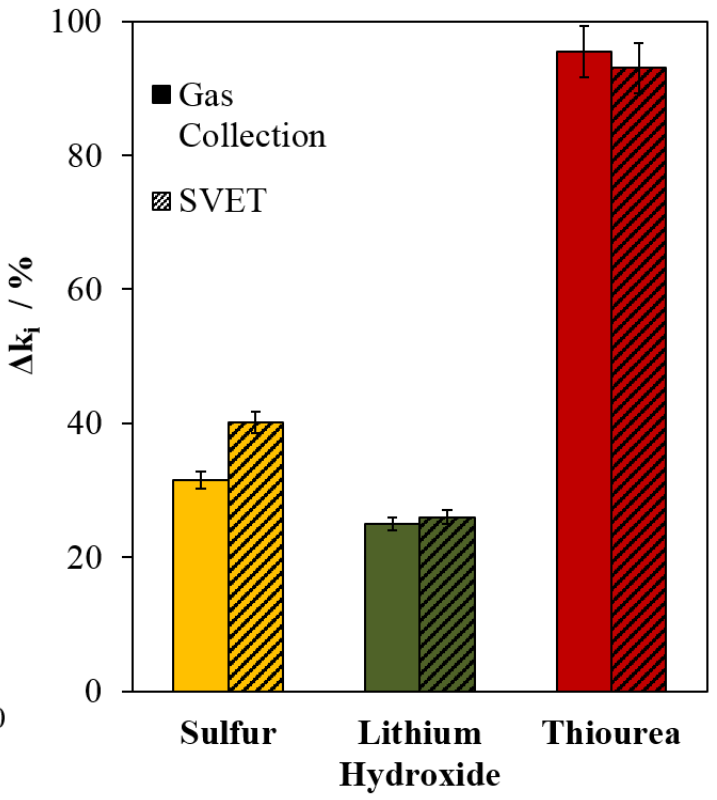

Figure 74 a) Plots showing the amount of hydrogen evolved from an iron electrode charging potentiostatically at $-1.4 \mathrm{~V}$ vs. $\mathrm{Hg} / \mathrm{HgO}$ when fully immersed in $\mathrm{KOH}$ $(30 \%$ w/v solution) measured using the SVET where i) no inhibitor, ii) $\mathrm{LiOH}$, iii) $\mathrm{S}$ and iv) Thiourea are present in the electrolyte. b) Plot showing the change in hydrogen evolution rate $\left(\Delta \mathrm{k}_{\mathbf{i}}\right)$ of hydrogen evolved measured using the SVET and the volumetric technique

SVET has been used on planar electrodes with spatially resolved results showing agreement with traditional techniques.

\subsection{Non-planar Electrodes}

Previously, it was evidenced that the SVET has the capability to accurately examine planar iron electrodes during charging and the possibility of examining non-planar electrodes was discussed. To further advance this technique and show the potential to utilize the SVET in this manner, non-planar electrodes were investigated. 


\subsubsection{Half Ground Substrate}

Using the method described in 2.1.1, a sample was prepared such that half the surface was ground and the remaining half of the sample was as received (unpolished) (Figure 75 and Figure 76). The SVET was calibrated (2.2.5) and a 3D height scan (2.2.3) was performed.

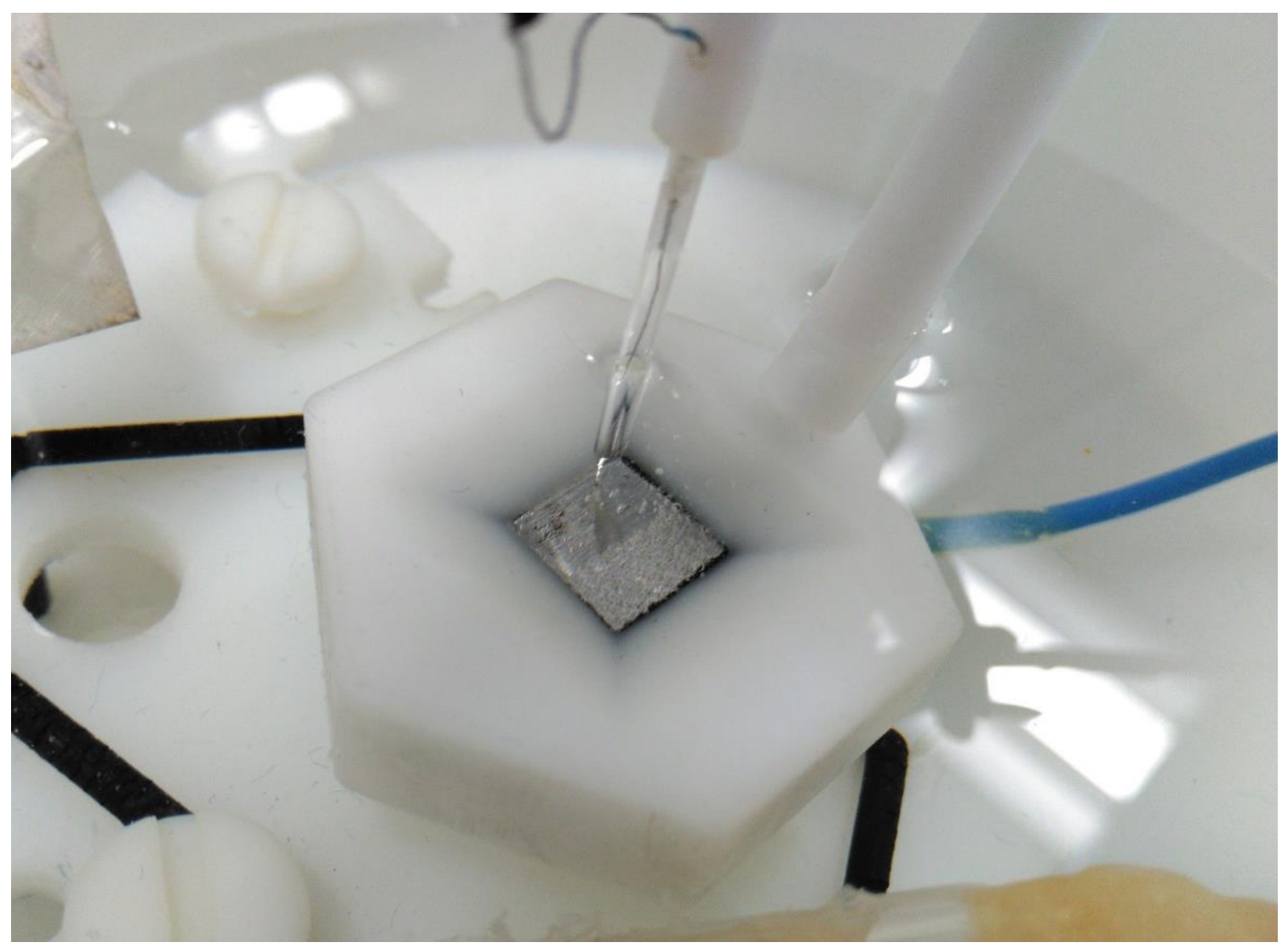

Figure 75 Image of the SVET setup for the half ground sample 


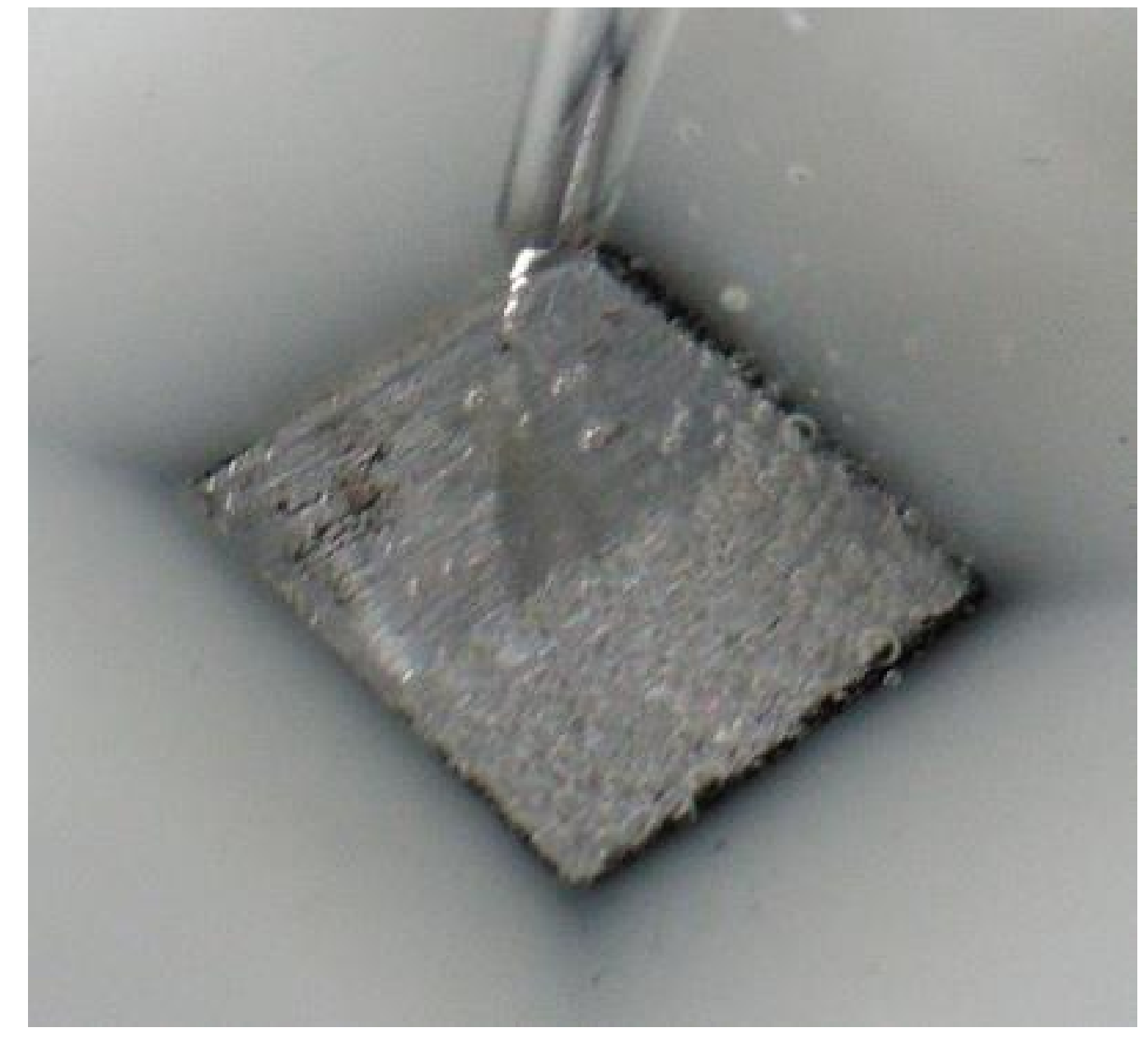

Figure 76 Detail view of experimentation showing apparent increased hydrogen evolution on ground area of sample

\section{Verification of 3D height scan}

In order to confirm that the SVET was capable of accurate surface measurement, surface analysis was completed using white light interferometry (WLI) (2.5).

This was necessary to ensure that the surface topography of the ground sample was suitable for 3D Height scanning. If peaks and troughs were present, but were not separated by a significant distance and the probe was unable to penetrate the troughs due to its physical size, then the SVET-measured 3D height scan would not be an accurate reflection of the surface topography. In this instance, it was important to consider the size of the peaks and troughs, in order to determine the effect they would have on SVET scanning. 
The as received area of the sample had a roughness of $459.28 \mathrm{~nm}$ (Figure 77) with sample tilt removed within Vision $32 \AA$, as measured on a 1.7 × $2.3 \mathrm{~mm}$ randomly selected sample area. The rolling direction was clear on this sample.

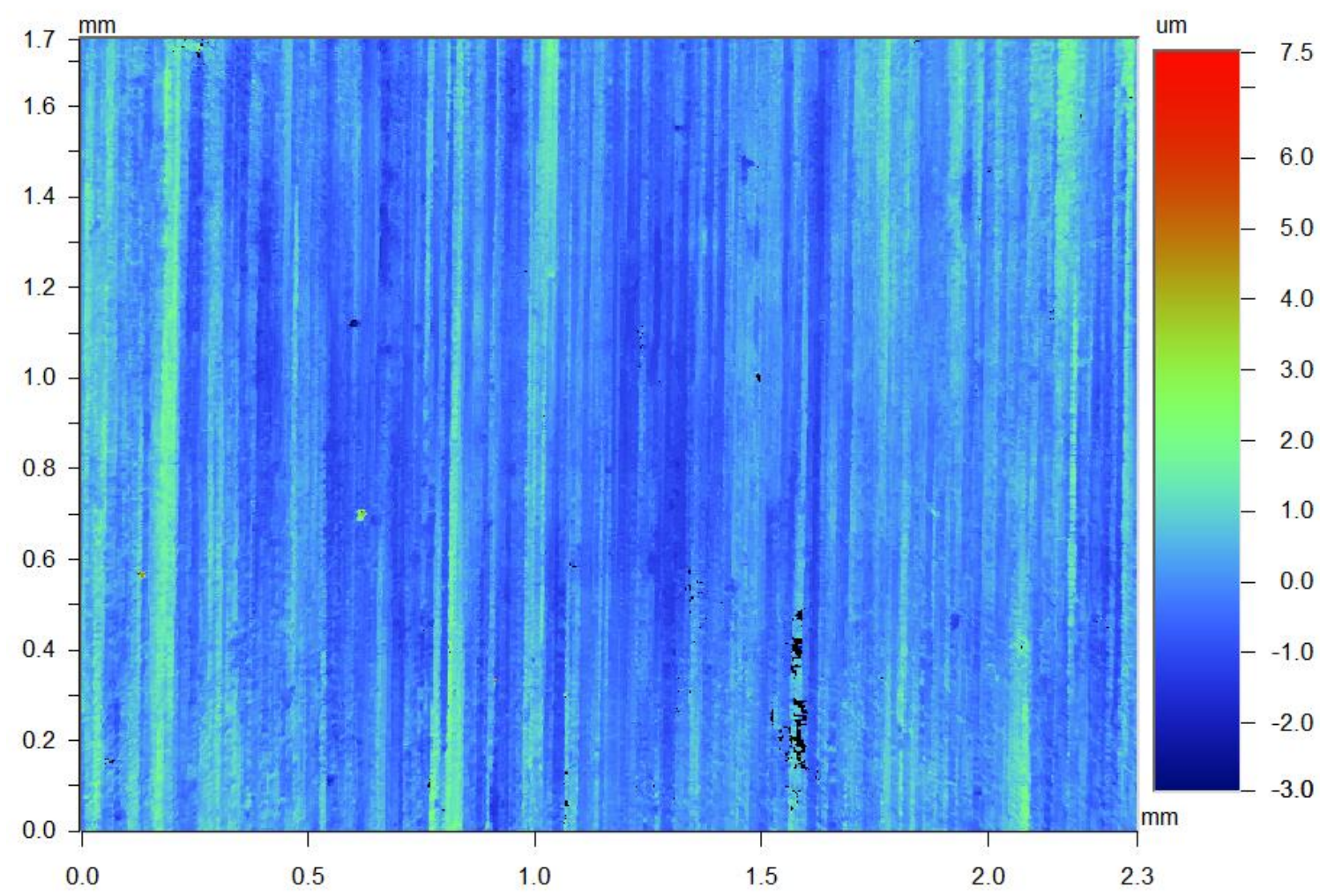

Figure 77 WLI scan of as received surface of steel sample

A line scan of a randomly selected section of the as received area of the sample shows a maximum vertical difference of only $2.600 \mu \mathrm{m}$ within a distance of $0.504 \mathrm{~mm}$ (Figure 78). Due to the motorized hardware which manipulates the probe, the SVET vertical accuracy is $2.5 \mu \mathrm{m}$, so this surface roughness is not considered significant. 


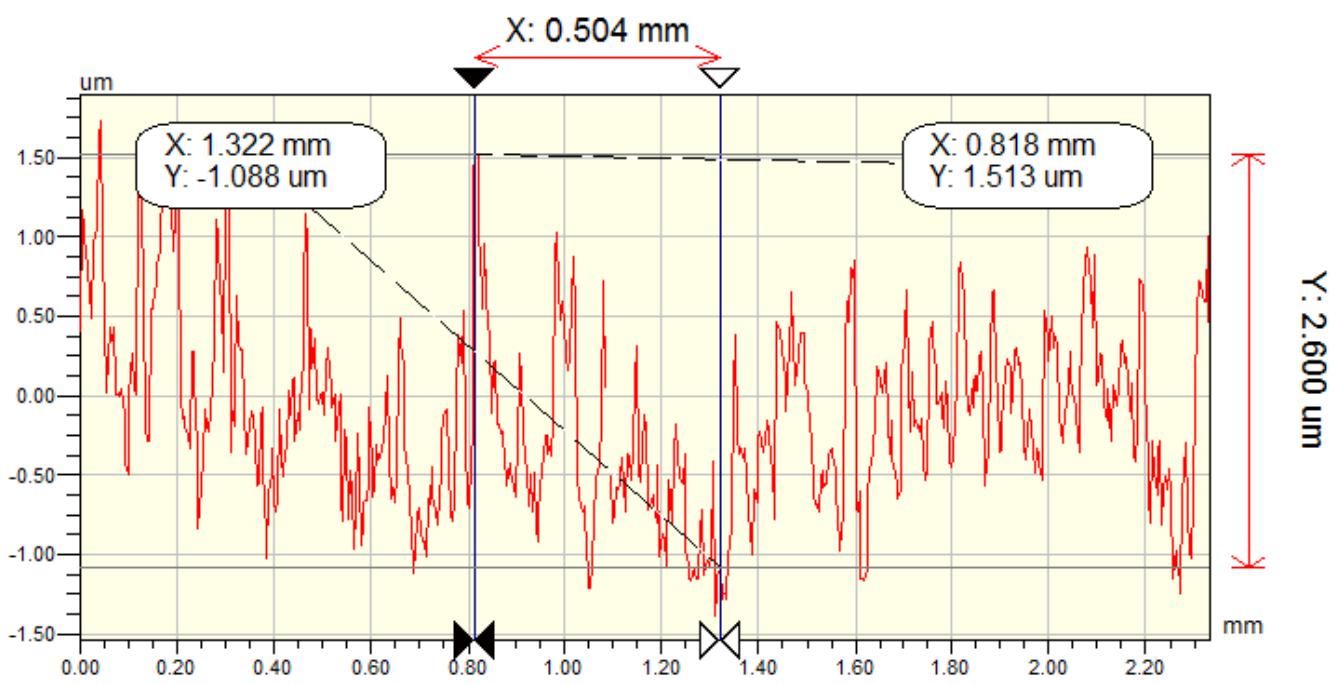

Figure 78 Line scan showing measurement between a peak and a trough on as received surface of steel sample

On the area of the sample which had been ground, Ra was measured at $1.11 \mu \mathrm{m}$ (sample tilt removed), over double of the as received surface. Despite the grinding, the rolling direction was still clearly visible (Figure 79).

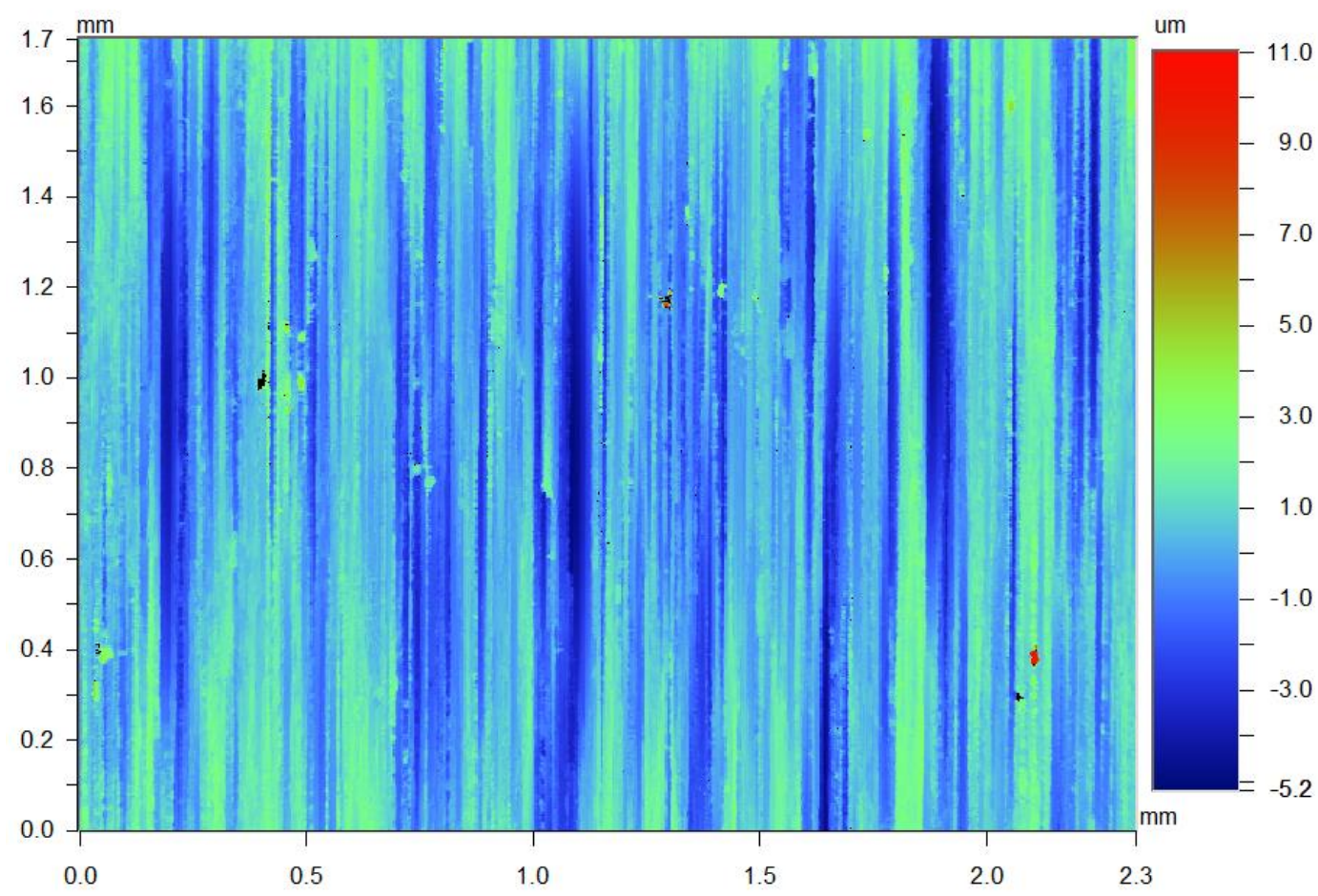

Figure 79 WLI scan of as ground surface of steel sample 
Whilst roughness was approximately doubled by the action of grinding the surface of the sample, it is not considered to have produced an effect which would cause the height scan to be invalid. It is assumed that a roughness of $5.4 \mu \mathrm{m}$ is only slightly larger than the height scan error and not large enough to result in a significant effect on data readings (Figure 80 ).

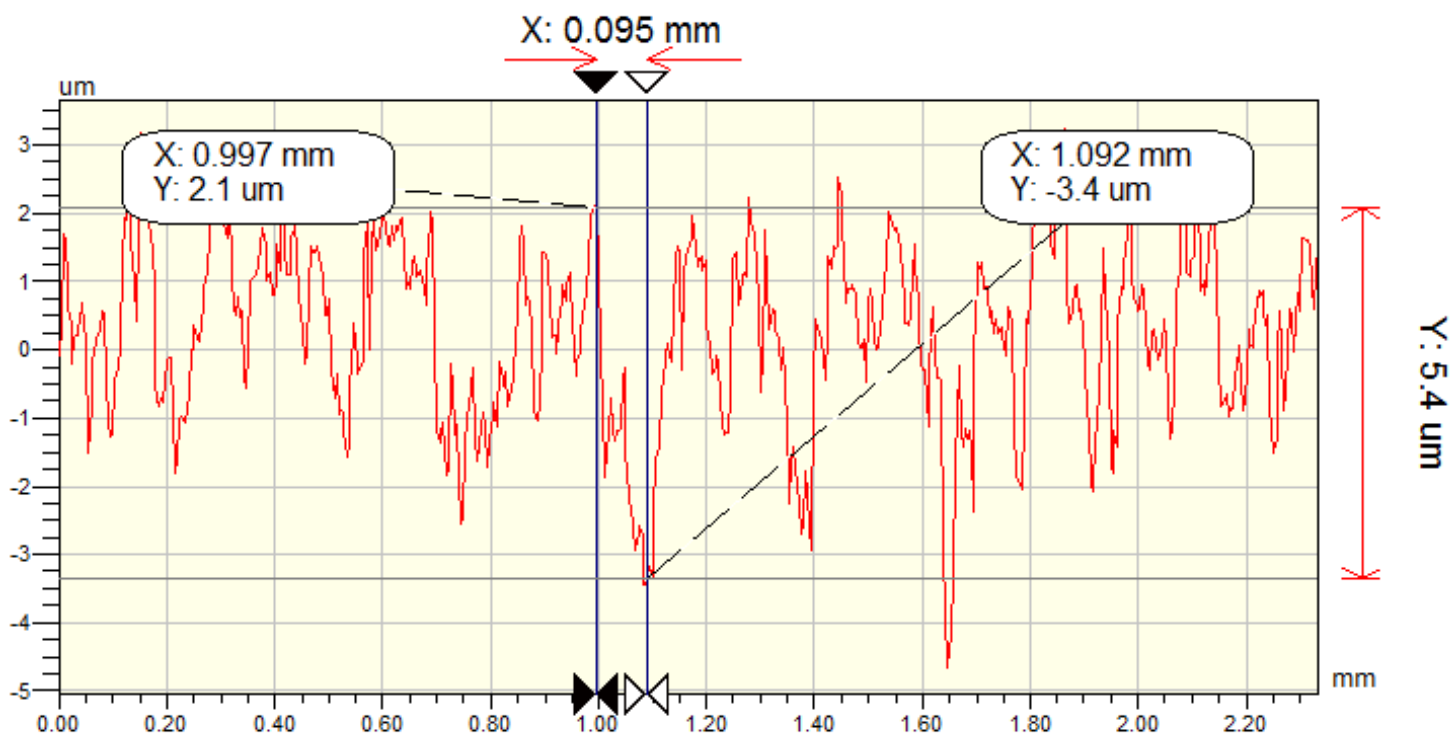

Figure 80 Line scan showing measurement between a peak and a trough on ground surface of steel sample

It is concluded from the WLI recordings that the height scan is still accurate for a sample with this surface topography.

\section{SVET height scan of half ground sample}

The 3D height scan of the sample which is ground on one side shows only small variations in height across the sample area (Figure 81). This is expected and agrees with the data recorded during the WLI investigation. 


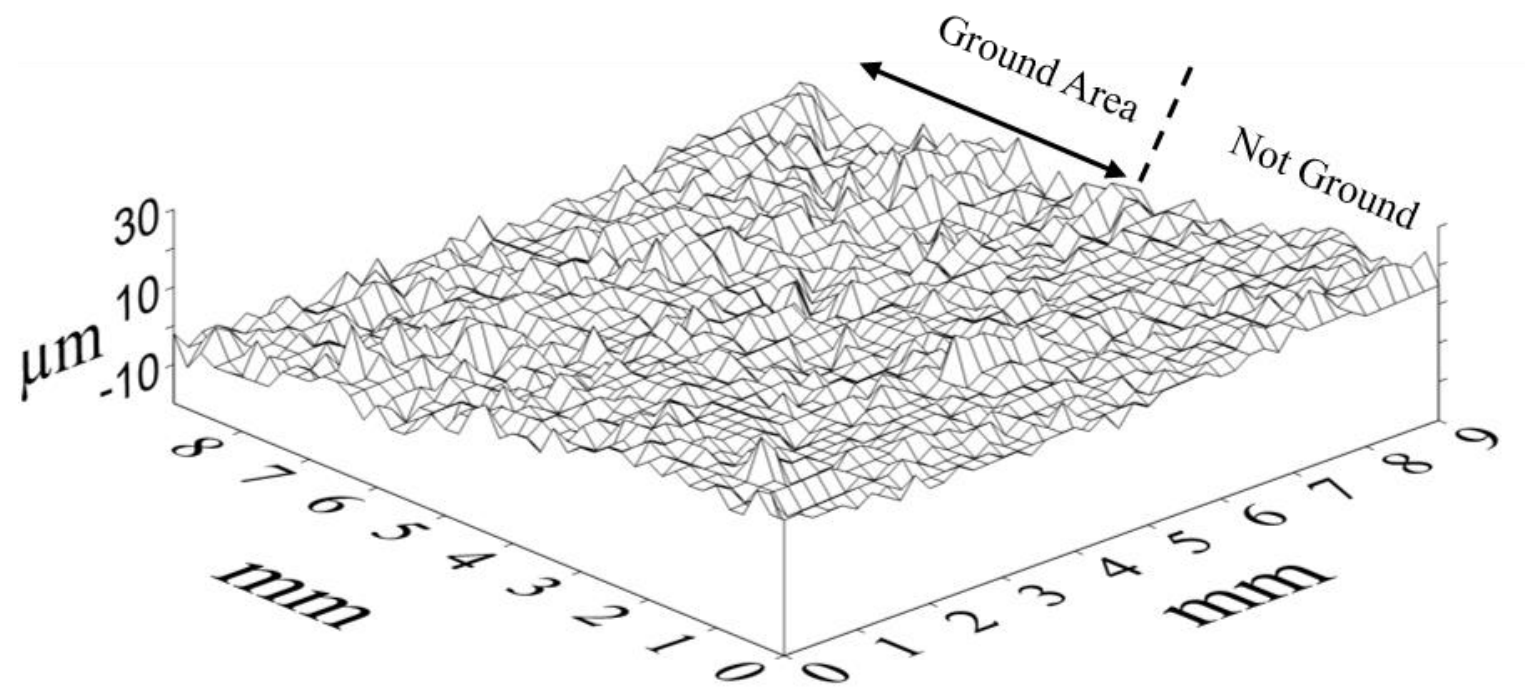

Figure 81 Height scan of half ground sample with annotation for the area which had been ground prior to height scan

\section{SVET data of half ground sample}

Since the sample is no longer considered to be uniform, analysis of the total charge consumed during experimentation is no longer applicable. Instead, Surfer ${ }^{\circledR} 10$ (Golden Software) was used to plot the data as false-color contour maps, as described previously (2.2.6). Figure 82 shows the sample on its $1^{\text {st }}$ SVET scan. The lower half of the image is the area of the sample which had been ground prior to testing. 


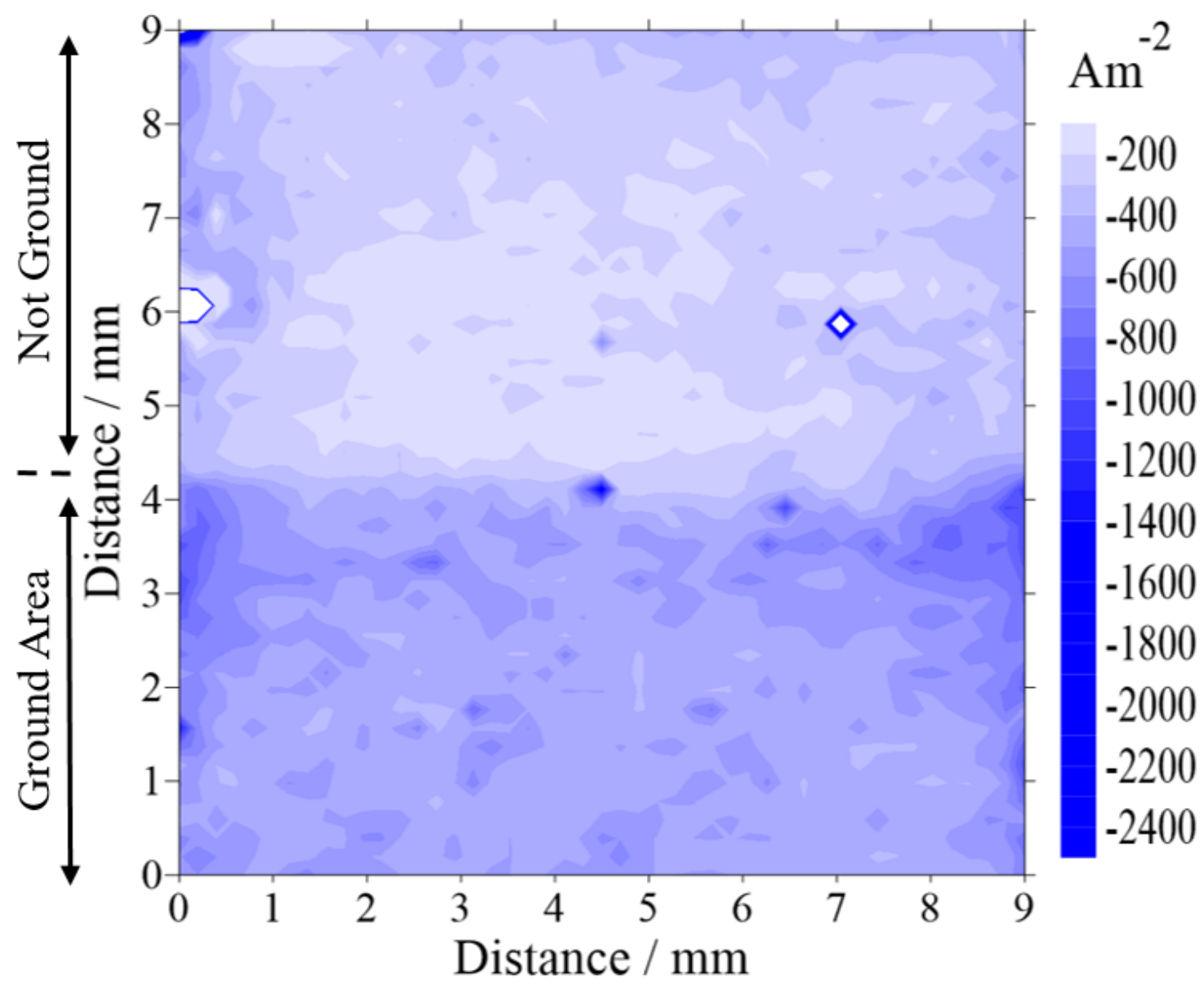

Figure 82 SVET (scan 1) of half ground substrate (ground area lowermost)

The ground area (lower half of image) is, on the whole, darker than the top half (unground substrate). This translates as the sample having a larger amount of cathodic activity occurring on the area which has been ground. The author assumes that this is due to the larger surface area resulting in more material available to support the hydrogen evolution reaction, however no additional analysis has been completed to confirm this. WLI could have been used to calculate the sample surface area, however the sample scans shown previously were not of sufficient size to produce useful data.

On this scan there are two points of interest, $(0.2,6.0)$ and $(7.5,5.8)$. Data is not recorded clearly at these points. It is assumed to be due to a bubble hitting the probe as measurement was taking place. The SVET is unable to record data when the probe is not immersed, as the probe must pass through a current flux. 


\subsubsection{Substrate With an Area of Sintered Iron}

Using the method described in 4.8, a sample was prepared such that a small proportion of the sample area was composed of Iron ink C2150413D1 (Gwent Electronic Materials Ltd.) mixed by hand with Expancel 950 DU 80 blowing agent (Boud). This produced discrete 'mounds' of iron, showing an extreme change in surface topography when compared with the half ground substrate. This sample was investigated in order to show the potential of the SVET for examining complex electrode surfaces. A photograph of this sample is visible in Figure 83.

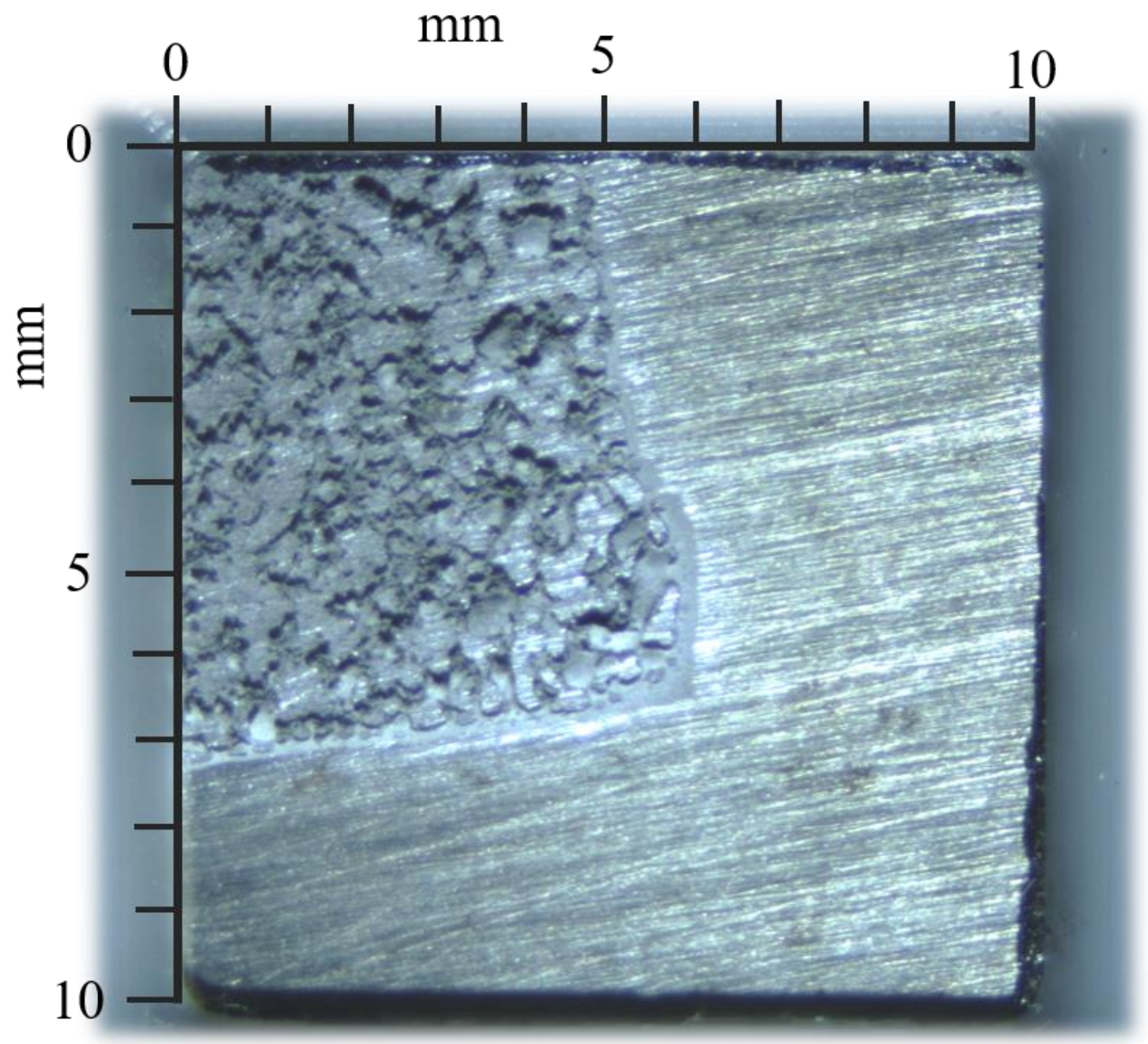

Figure 83 Macro image of substrate with an area of sintered iron 


\section{Verification of 3D height scan}

As in 5.5.1, height scan verification was completed using WLI. On this sample, the entirety of the substrate has been ground, prior to coating with iron and sintering. It has previously been demonstrated that the ground substrate is compatible with the SVET 3D height scanning.

The sintered surface is broken up due to the use of the blowing agents. Some areas are uncoated and the substrate is visible, some areas contain 'lumps' of sintered iron. Figure 84 shows the height of these features, as identified by white light interferometry.

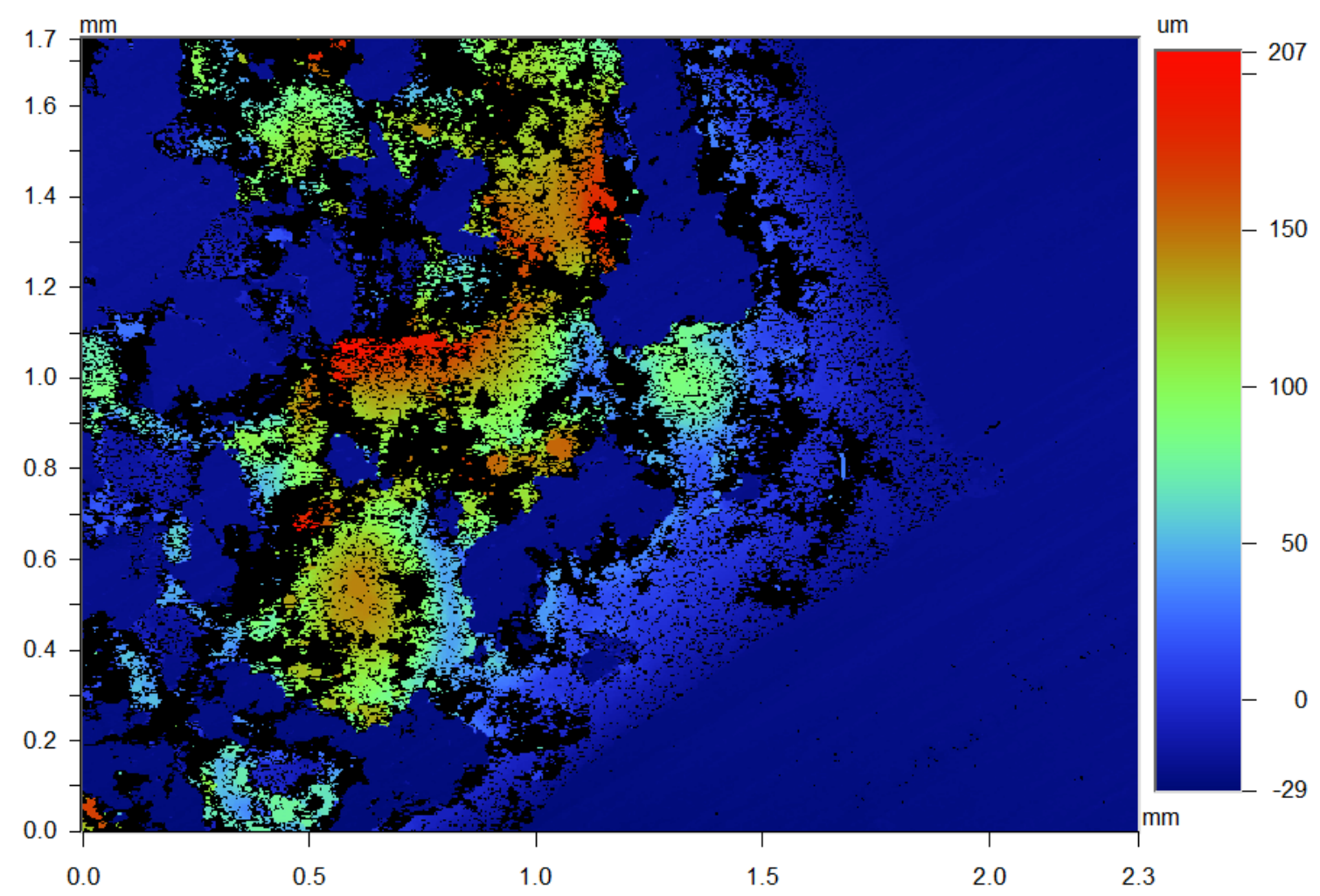

Figure 84 WLI scan showing structure of sintered iron (left) on substrate (right)

In order to assess the compatibility with SVET height scanning, the distance between features needs to be examined. 

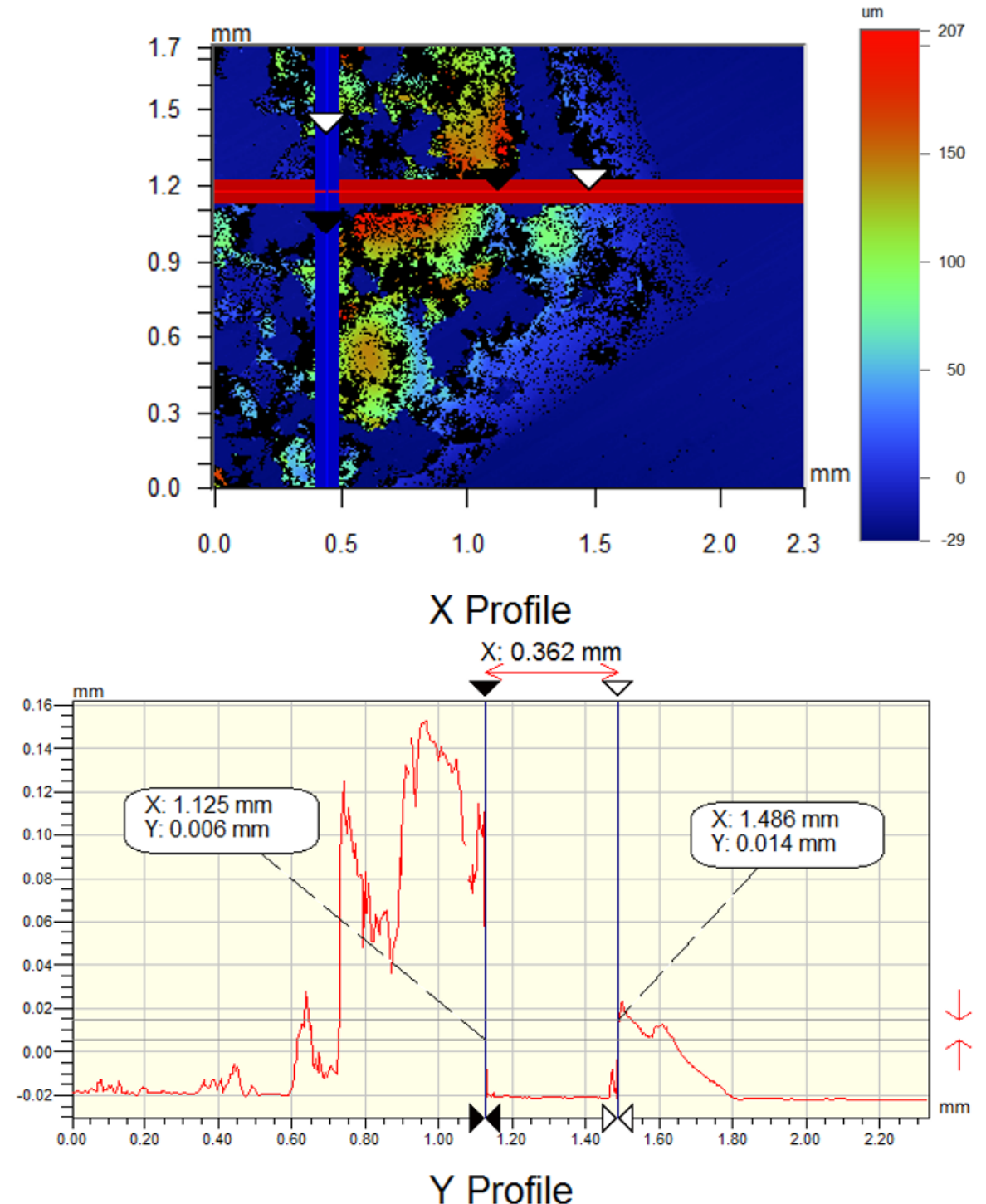

$\prec$
$\circ$
$\circ$
0
0
콩

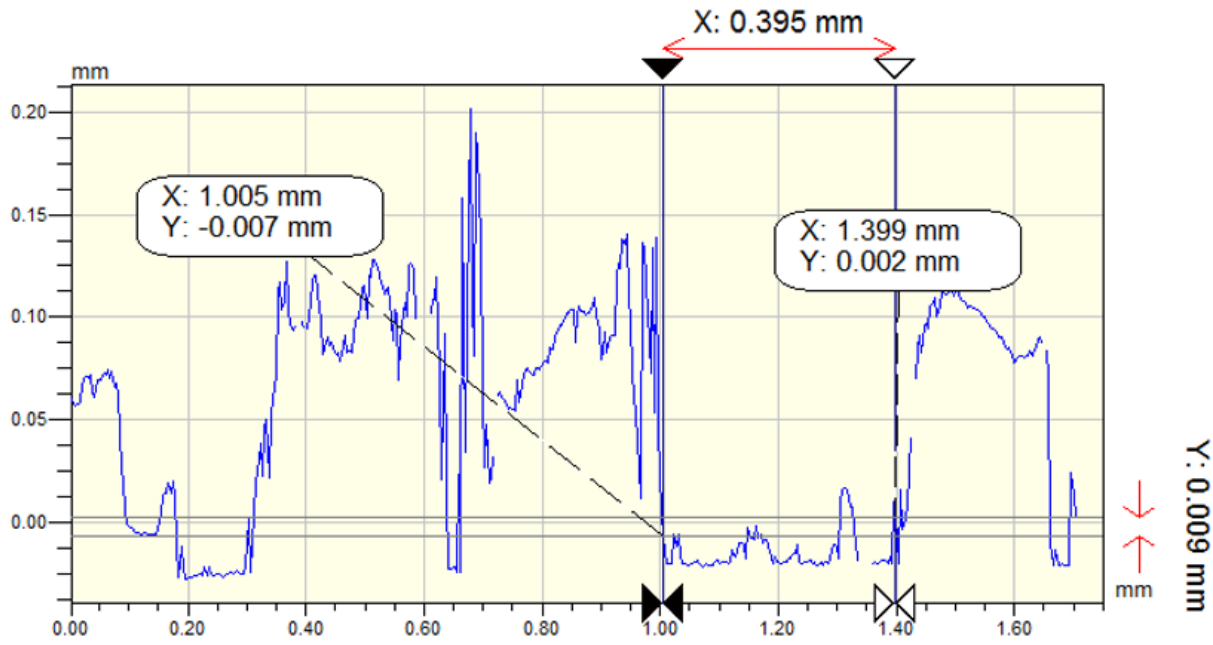

Figure 85 Cross section view of two sintered iron troughs 
Figure 85 shows the size of various features on the sintered area of the iron electrode. On the $\mathrm{X}$ profile, one feature was highlighted. This trough has occurred where no sintered iron is present and the substrate surface is visible. The width is measured at $0.362 \mathrm{~mm}$, which is slightly larger than the width of the glass SVET probe (5.3.2), suggesting that the probe is able to fit inside the pit for height scan measurements. Additionally, the small feature on the right of the $\mathrm{X}$ profile is measured at $39 \mu \mathrm{m}$ height from the substrate, so any errors resulting from the probe failing to observe the marked trough will be minimal.

On the Y profile, a large trough is visible with a width of $395 \mu \mathrm{m}$ in the $\mathrm{Y}$ direction. This is large enough for the probe to fit, allowing measurement to the bottom of the trough, excluding the features in the trough of $35 \mu \mathrm{m}$ height.

Overall, it is concluded that reasonable accuracy is possible through use of the SVET with a 3D height scan. Some errors, such as those described by Searle [178], will result from small features, such as narrow troughs/valleys and sharp points. Use of the blowing agent was crucial to providing a structure suitable for 3D height scanning.

\section{Height scan of substrate with area of sintered iron}

A wireframe of the SVET 3D height scan of the sintered iron sample can be seen in Figure 86. This shows that the majority of the surface of the sample was recorded as having very little height change, in agreement with Figure 83. On the area where sintered iron is present, it is clear that a range of heights were recorded. If the SVET probe was unable to fit into the narrow troughs/valleys, then the entirety of this area of the sintered iron would have been recorded as being raised - this is not the case. 


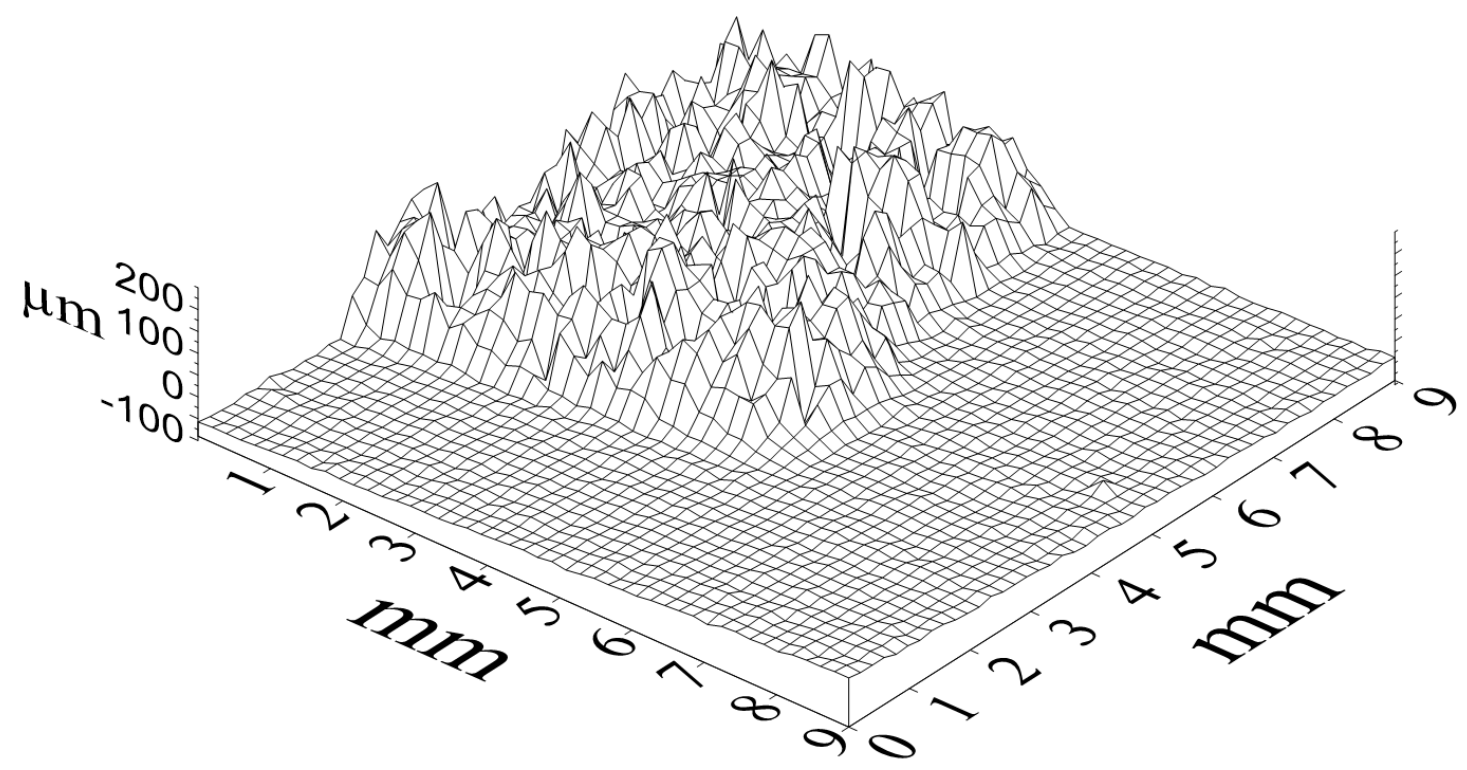

Figure 86 Wireframe representation of 3D height scan performed by SVET

The clear evidence of valleys/troughs in the height scan data was considered to prove that the height scan for the sintered iron sample was valid.

\section{SVET data of substrate with area of sintered iron}

A data scan of the sintered iron sample was performed with an impressed potential of -1.4 $\mathrm{V}$ vs. $\mathrm{Hg} / \mathrm{HgO}$. Figure 87 shows the current density map which was recorded in scan 1 of the SVET probe. A range of current densities are recorded across the sintered area of the sample and relative to this, the uncoated substrate appears to be mostly homogenous. 


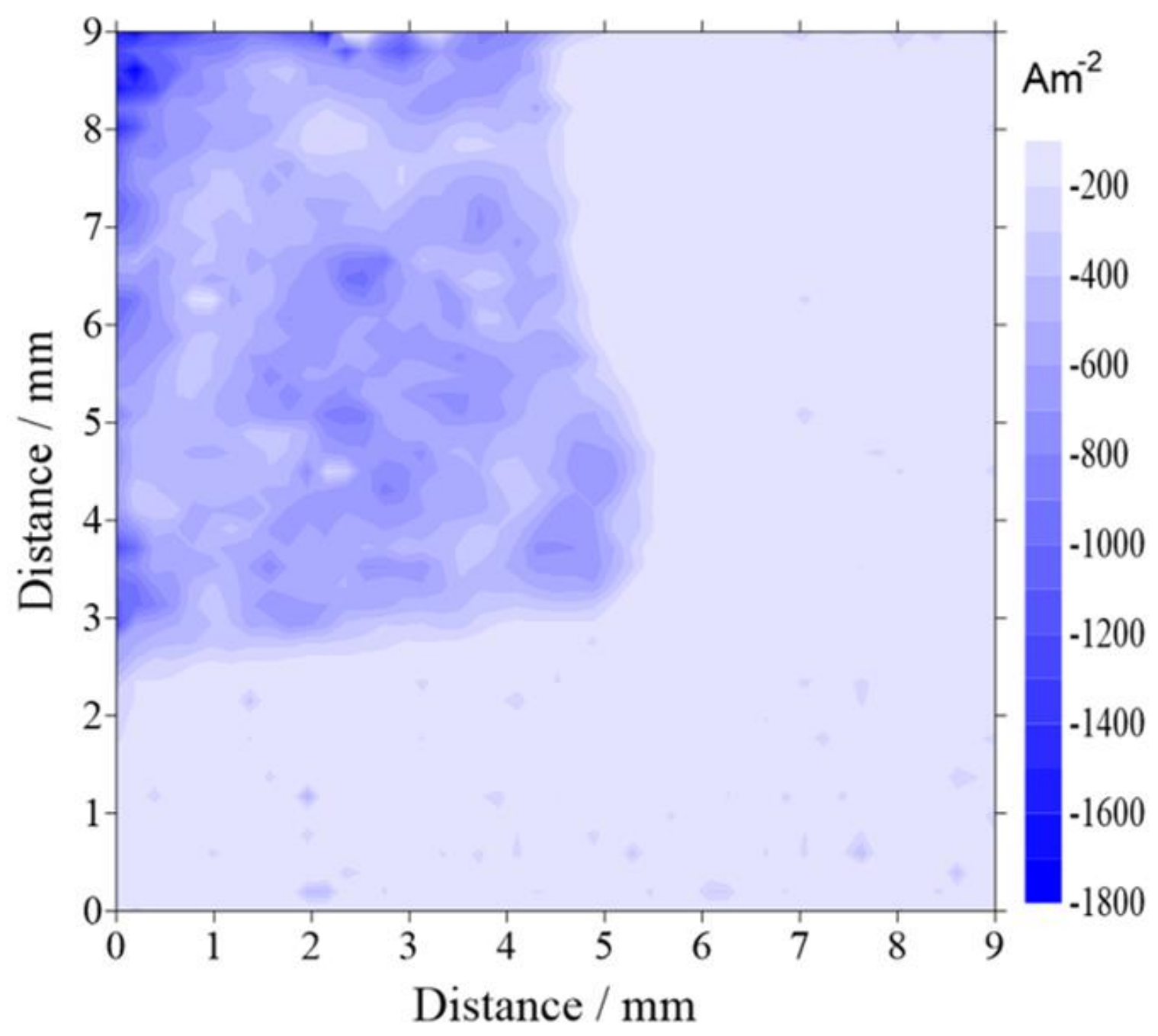

Figure 87 Current density as recorded by the 3D SVET, scan 1

In several areas on the uncoated substrate, points of data are observed. It is possible that the false color map used has exaggerated the significance of these features slightly, yet they are likely to be errors as shown previously in Figure 82. 


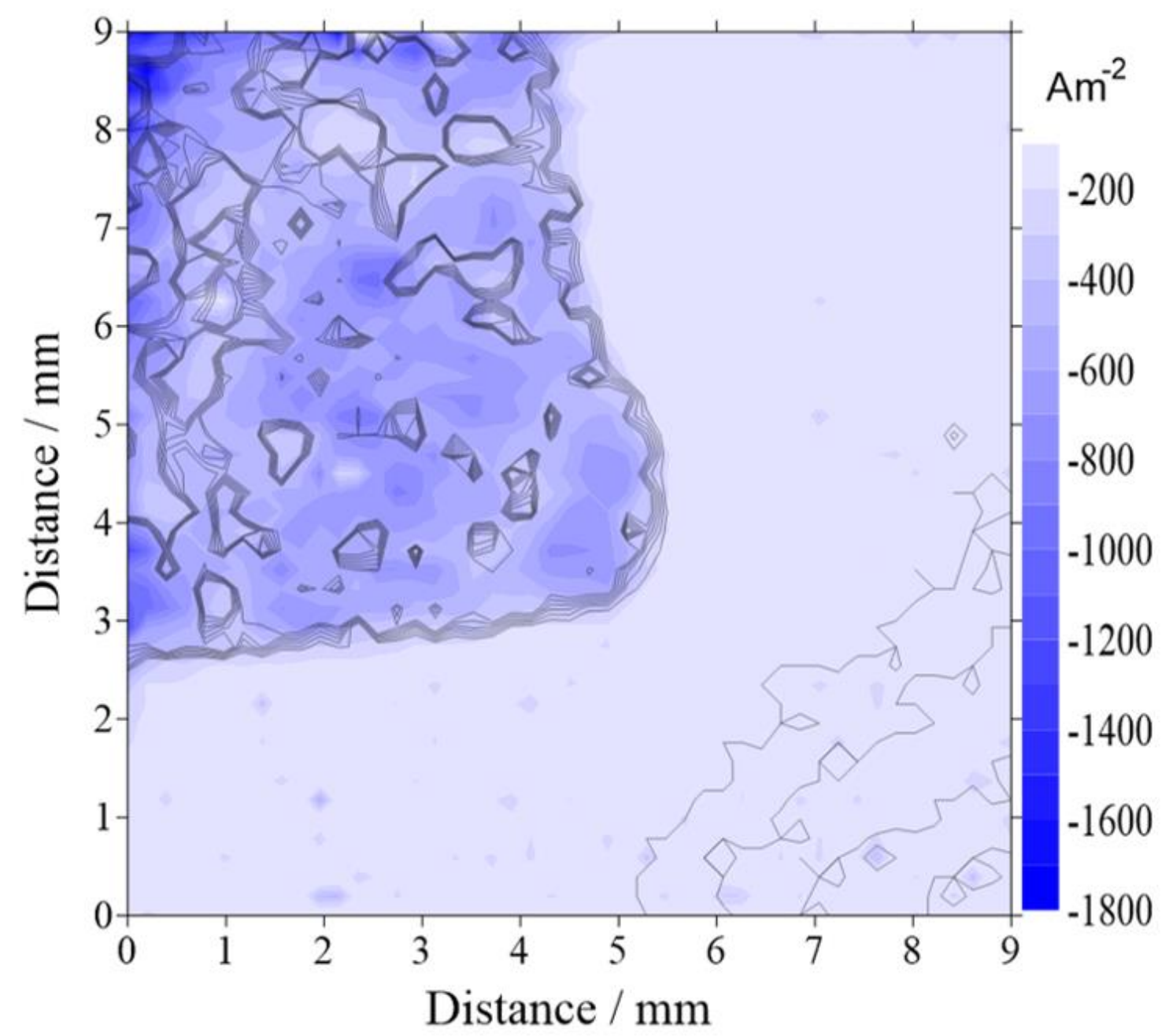

Figure 88 Contour map showing current density as recorded by the 3D SVET (colored shading), with overlaid contour lines (grey solid lines) showing regions of lower height as recorded by the height scan

In Figure 88, the height scan data has been overlaid as contour lines on the data scan that was shown in Figure 87. This allows for further investigation into the accuracy of the data scan. It is clear that there are multiple areas where low current density is recorded and these also correspond to valleys/troughs on the height scan. This suggests that the probe is able to penetrate these troughs, however it does not confirm that the data produced from scanning over these troughs is an accurate representation of the surface activity in this location. An alternative view of this data is shown in in Figure 89. 


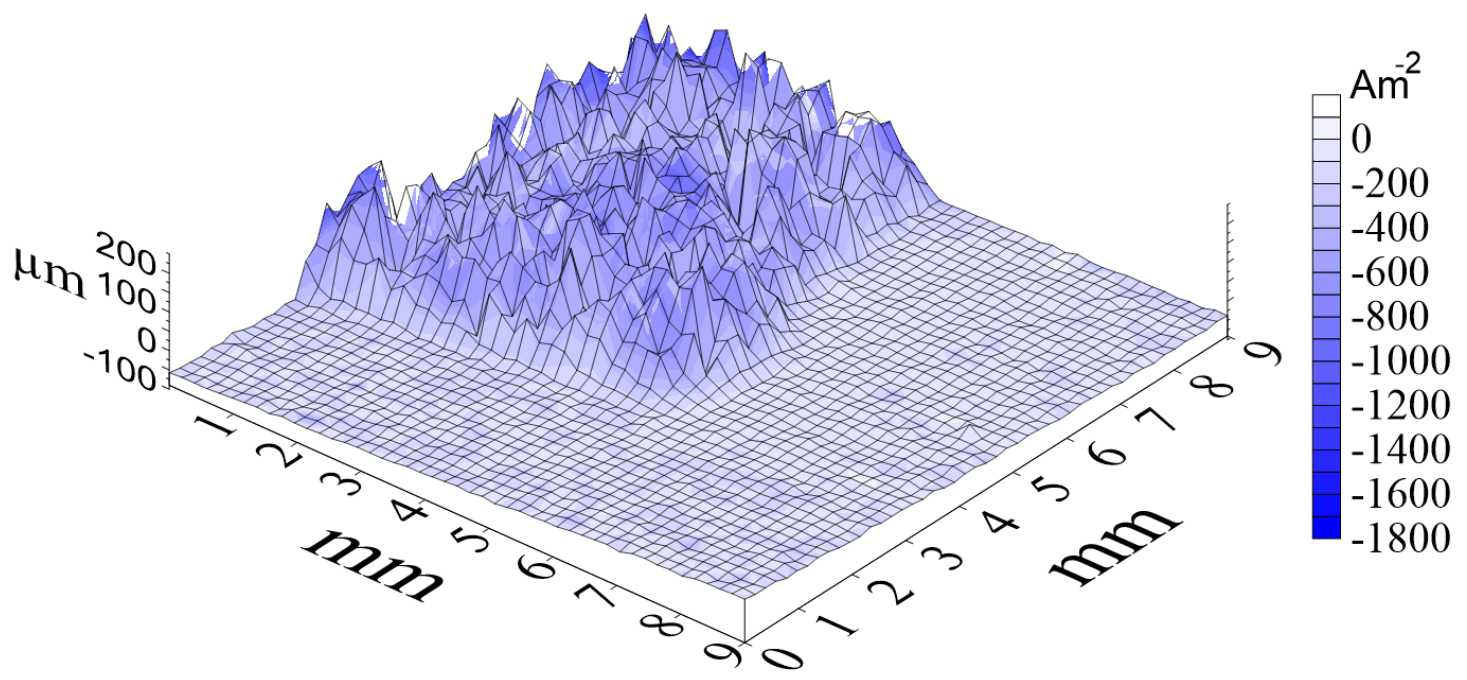

Figure 89 3D wireframe with SVET-derived current density (scan 1) overlaid

\subsection{Conclusions}

The Scanning Vibrating Electrode Technique has been used for the first time in energy storage, as a means of analyzing electrochemical storage devices in situ. This was enabled by this researcher's design and manufacture of sample holders and related equipment.

The SVET was used to demonstrate the effective reduction in hydrogen evolving from a charging iron electrode when fully immersed in $\mathrm{KOH}$ electrolyte containing additions of inhibitors. A ranking order was established where thiourea $>$ sulfur $>$ lithium hydroxide.

These results are in agreement with verification experiments carried out using a volumetric measurement technique and cyclic voltammetry. SVET has been shown to be accurate in quantitative evaluation and offer a significant advantage over conventional techniques in its spatial resolution and ability to measure heterogeneity and transient reactions. The SVET thus shows potential for further investigation for aqueous energy storage electrochemistry, both for iron electrodes and other chemistries.

Additionally, this technique is further expanded in order to be utilized on non-planar electrodes, a previously-impractical environment. It has been shown that electrodes with a half ground surface can be accurately analyzed. When electrodes which contain a 
physically rough area of sintered iron are analyzed, it appears that the height scan is correctly detecting large features, when compared to data gathered by white light interferometry. SVET measurements of extremely rough samples, such as the substrate with an area of sintered iron is considered to be sufficient quality for qualitative analysis of electrodes of similar surface topography.

Development of the SVET for use in aggressive alkaline conditions is important as it allows for the scanning electrochemical technique to be utilized in the investigation of iron in an energy-storage context. Previously, it was not possible to operate the SVET in these conditions, however this work has shown the potential for the technique to be expanded to the analysis of alkaline battery electrodes. 


\section{Chapter 6: A Scanning Kelvin Probe Study of the Hydrogen Absorption on the Iron Electrode}

\subsection{Introduction}

In continuation of the scanning electrochemistry work discussed in the previous chapter, the Scanning Kelvin Probe is used to investigate the mechanism of action of inhibitors. Whilst the different inhibitors function in different ways, the mechanisms are unclear. It was not possible to determine the mechanism with the Scanning Vibrating Electrode Technique as used previously.

Thiourea is studied mechanistically, following on from its strong hydrogen evolution inhibiting in the previous chapter. Additionally, Benzotriazole (BTA) is an interesting inhibitor which has not been used so far by this researcher. Traditionally, it is used as a corrosion inhibitor on Copper alloys [124]-[127]. Colleagues within my research group have reported interesting findings with benzotriazole on ferrous substrates, however, these have not been published at the time of the author's writing. Research involving benzotriazole and steel is reported in literature [132], [213], [214] and benzotriazole has also been used in Li Ion battery research [215].

The use of benzotriazole on a wide range of metallic substrates encouraged the author to investigate its effects on hydrogen evolution on iron substrates.

Initially, the Scanning Vibrating Electrode Technique was utilized in the same manner as in Chapter 5, with benzotriazole incorporated as an additive in the electrolyte. This allowed a comparison of performance of the two inhibitors, such that a rank could be established.

However, in order to build on the research using the Scanning Vibrating Electrode Technique, further experimentation was required to understand the mechanism of inhibition. A powerful effect had previously been observed with Thiourea as a soluble inhibitor for hydrogen evolution (5.4). A mechanism for the action of Thiourea was proposed in research reported by Amokrane et al [123]. This study also used benzotriazole and proposed a contrasting mechanism for the two inhibitors. 
Hydrogen evolution is a multi-step process, where atoms of hydrogen diffuse within the lattice of the material before recombining on the surface and forming a hydrogen molecule. A study by Amokrane et al reported that benzotriazole inhibits penetration of $\mathrm{H}_{2}$ into the metal by reducing the rate of the Volmer reaction, as shown in Equations 6.1-1 and 6.1-2:

$$
H^{+}+e^{-} \rightarrow H_{a d}
$$

Whereas, thiourea increases penetration of $\mathrm{H}_{2}$ into the metal by reducing the rate of the recombination reaction:

$$
H_{a d}+H_{a d} \rightarrow H_{2 a d}
$$

It is known that the presence of atomic hydrogen in a metal foil results in variations in the local Volta potential [173], [174]. The Scanning Kelvin Probe (SKP) has previously been used in similar experimentation. The Volta potential, also known as the Volta potential difference or contact potential difference, is the potential between two metals which are in electrical contact. Heterogeneity can occur across the surface of an electrode due to locational effects such as inclusions, surface cleanliness or, in this case, variations in the oxidation state of the surface oxide film [216]. By scanning the electrode surface, a map of the local Volta potential can be produced and the heterogeneity measured.

Experimentation was devised where the SKP was used to map the changes in Volta potential in regions of iron which had undergone charging in $\mathrm{KOH}$ solution with additions of thiourea or benzotriazole as inhibitors. This was compared to areas of the same electrode which had not undergone hydrogen evolution. Contrast in the methods of inhibition were expected to result in variations in the amount of atomic hydrogen adsorbed in to an iron foil and subsequently, the local Volta potential (1.7.3). This would allow for the mechanisms proposed by Amokrane et al to be verified.

\subsection{Ranking Performance of inhibitors using SVET}

Initially, the SVET is used to understand the performance of thiourea and benzotriazole as hydrogen evolution inhibitors. 


\subsubsection{Materials}

In line with previous work, discs of iron (99.5\% purity), $1 \mathrm{~mm}$ thickness were sourced from Goodfellow Metals Ltd. Iron samples were polished to a mirror finish and degreased in ethanol immediately prior to testing. Potassium hydroxide was used at a concentration of $30 \% \mathrm{w} / \mathrm{v}$ with $0.1 \mathrm{M}$ additions of thiourea and benzotriazole. Iron was used to represent the active material in the iron electrode. The polished surface was included to reduce variation between samples.

\subsubsection{Method}

SVET experimentation was conducted as per previous experiments in Chapter 5. Scans were completed in both the presence and absence of inhibitors. Further details on this technique, including the set-up and calibration procedures and data analysis methodology can be found in 2.1 .

\subsubsection{Results and Discussion}

Hydrogen evolution was measured using the SVET (Figure 90). Additions of thiourea and benzotriazole both show a reduction in the hydrogen evolution rate when compared to the uninhibited example. Thiourea achieved a $92 \%$ reduction in hydrogen evolution rate after $15 \mathrm{~h}$ and benzotriazole a $66 \%$ reduction in the same period. It is determined that thiourea is more effective at blocking the hydrogen evolution reaction than benzotriazole. Additions of both inhibitors achieve a decrease in the amount of hydrogen evolved relative to the uninhibited sample. 


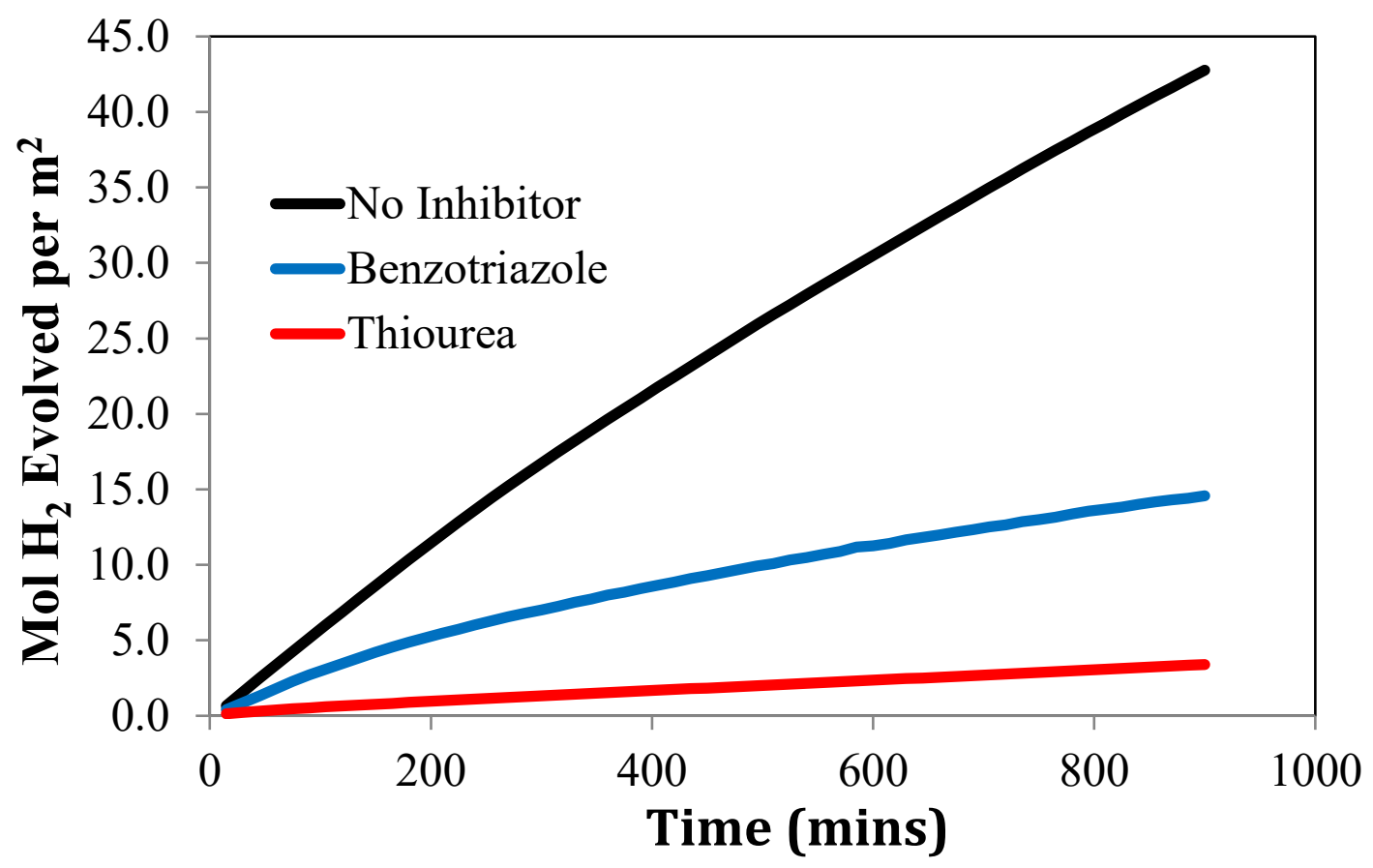

Figure 90 Plot showing amount of hydrogen evolved from an iron electrode charging potentistatically at $-1.4 \mathrm{~V}$ vs. $\mathrm{Hg} / \mathrm{HgO}$ when fully immersed in $\mathrm{KOH}$ $(30 \% \mathrm{w} / \mathrm{v})$ with inhibitors of thiourea $(0.1 \mathrm{M})$ and benzotriazole $(0.1 \mathrm{M})$

Verification with a traditional technique is not necessary as the accuracy of the performance of the SVET in these conditions was evidenced in Chapter 5. Cathodic current density maps (Figure 91) were examined for heterogeneity, however all results appeared homogeneous. Small erroneous readings have been recorded in some areas of the scans, this was previously assumed to occur when bubbles hit the probe exactly as measurement took place (Chapter 5.5). A higher cathodic current density is typically recorded at the periphery of the scan thought to be due to the effects of the sample holder on nucleation of bubbles of hydrogen. 

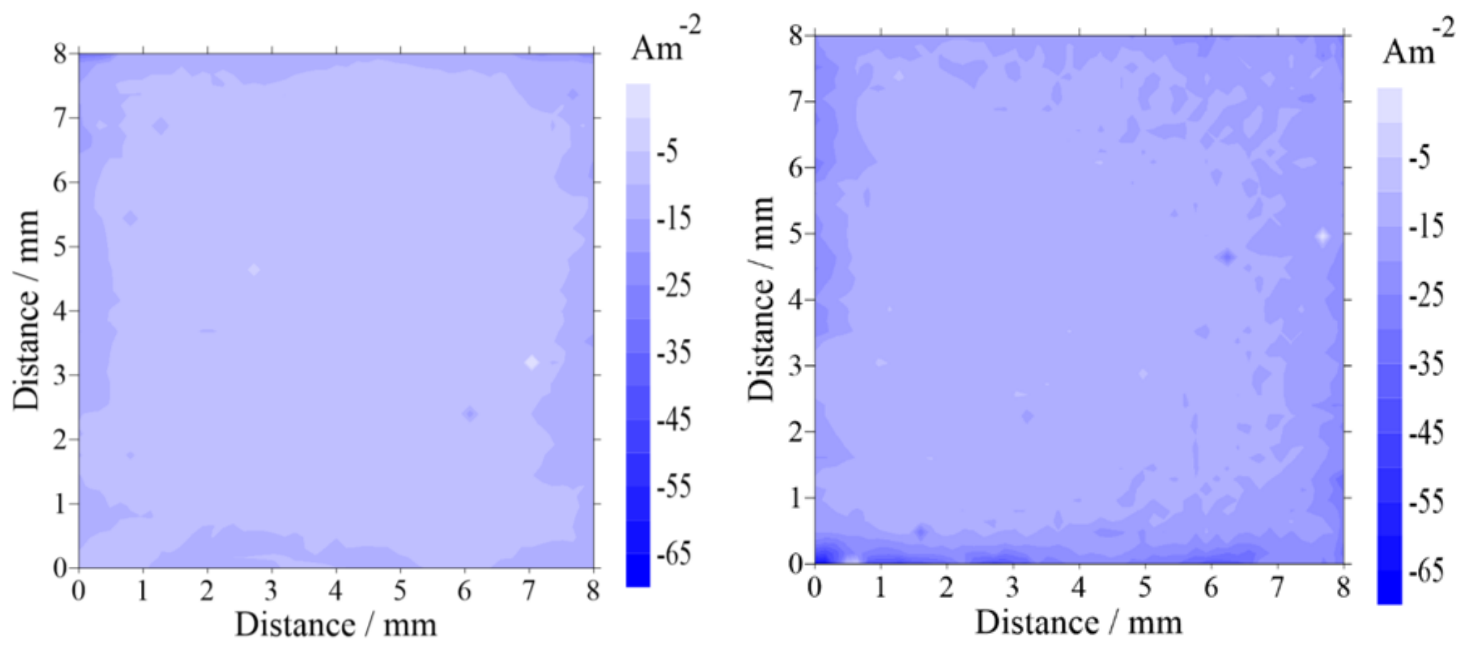

Figure 91 Cathodic current density maps from representative SVET scans. Left:

0.1 M thiourea addition repeat $5,24^{\text {th }}$ scan (6 hours). Right: $0.1 \mathrm{M}$ thiourea addition repeat $7,9^{\text {th }}$ scan $(2.25$ hours $)$

Through this work, SVET has established a ranking order for the inhibitors such that, thiourea $>$ benzotriazole $>$ no inhibitor. SKP analysis will now examine this relationship further.

\subsection{Mechanistic Study using SKP}

As the performance of the two inhibitors is now shown, the SKP will be used to investigate the different methods proposed in the introduction.

\subsubsection{Materials}

Iron foil (99.9\% purity), $0.1 \mathrm{~mm}$ thickness were sourced from Goodfellow Metals Ltd and prepared as described in Chapter 2.4. As with previous electrochemistry, iron was used as it is the same as the iron activity material used in an iron electrode. It was not technically appropriate use the steel substrate which was available from the project sponsor for this work.

Potassium hydroxide was used at $0.2 \mathrm{M}$ with $0.1 \mathrm{M}$ additions of thiourea and benzotriazole. All chemicals were supplied by Aldrich Chemicals. 


\subsubsection{Method}

It has previously been shown that the Scanning Kelvin Probe can be used to map Volta potentials resulting from hydrogen permeation. This is due to the effect of hydrogen on the oxidation state of the iron [173], [174].

Prior to SKP testing, samples were charged for varying time periods (1-20 minutes) in a $0.2 \mathrm{M}$ potassium hydroxide solution with either $0.1 \mathrm{M}$ thiourea or $0.1 \mathrm{M}$ benzotriazole added as inhibitors. A Solartron SL 1286 was used at $-1.4 \mathrm{~V}$ vs. $\mathrm{Hg} / \mathrm{HgO}$ and with a $\mathrm{Pt}$ counter electrode in order to evolve hydrogen from the surface. The sample was held in such a way that a circular area of $5 \mathrm{~mm}$ diameter on one face of the sample was exposed to the solution and the remainder of the sample was covered.

Immediately after the charging operation had completed, the sample was removed from the solution and rinsed with distilled water and cleaned with ethanol. The sample was then placed in the SKP chamber with the charged surface facing downwards, with the intention that SKP measurements would be detecting the effects of atomic hydrogen which had diffused through the bulk sample.

The SKP employed a $100 \mu \mathrm{m}$ diameter wire gold reference probe to scan across the sample at a fixed height of $100 \mu \mathrm{m}$. Further details of this equipment can be found in Chapter 2.4. Scans were carried out at room temperature and at a fixed $95 \%$ relative humidity. A scan area of $1 \mathrm{~cm}^{2}$ was used and this was centered on the reverse of the 5 $\mathrm{mm}$ diameter circle which had previously been evolving hydrogen. Volta potential maps were produced by scanning at 10 points $/ \mathrm{mm}$ at 60 minute intervals for 15 hours. Scans typically commenced within 10 minutes of stopping the hydrogen charging operation. Repeats were taken of all test points due to occasional erroneous results. Errors were typically caused by the cell leaking when immersed in solution, or damage to the electrode surface.

Efforts were made to align the setup of the experimentation with that performed by Amokrane et al, which mirrored previous experimentation by Gabrielli et al [218], with note to the different techniques in use. Thickness of the iron foil was altered as appropriate to the equipment (SVET/SKP) used in each experiment. Potassium hydroxide was used 
over sodium hydroxide due to its frequency of use in iron electrode batteries, however concentrations were comparable to experimentation conducted by these other researchers.

Additional details on the set up and operation of the SKP are available in Chapter 2.4.

\subsubsection{Results and Discussion}

As a result of scanning the probe over the sample surface, 2D maps were produced showing the $\mathrm{E}_{\text {corr }}$ vs. distance data. An example of this is included in Figure 92. Whilst hydrogen was previously evolved on the reverse face, the same area is visible on the face scanned with the SKP, showing that the atomic hydrogen has permeated through the iron foil. This type of image shows that there is a clear effect on the $\mathrm{E}_{\text {corr }}$ as a result of hydrogen evolution, however it is difficult to quantify the data in this format.

\section{$E_{\text {corr }}(m V$ vs SHE)}

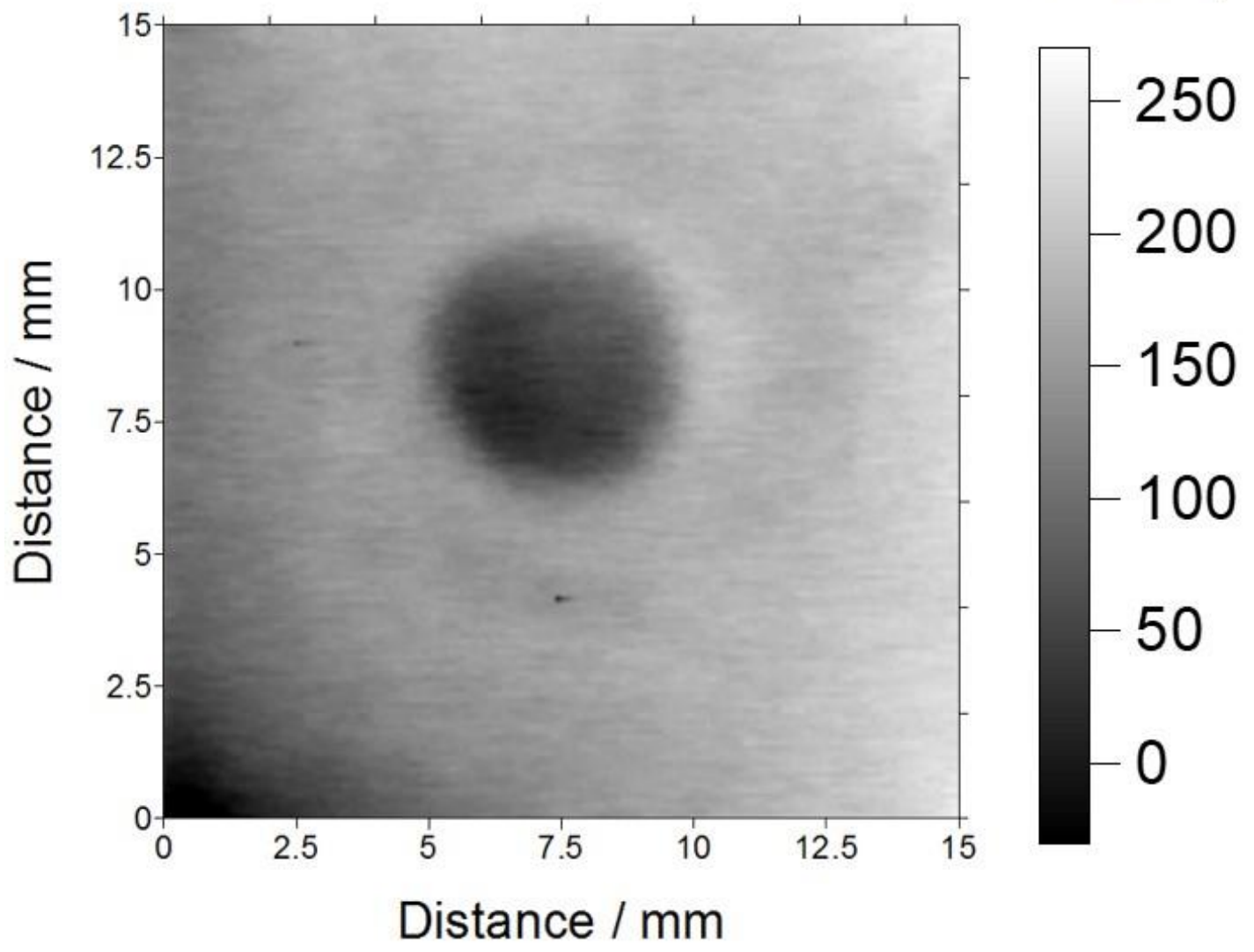

Figure 92 Example 2d map from SKP-derived data of a sample post charging 
To aid comparison, 1D line scan was used for further analysis. SKP was used to examine the Volta potential drop between the area which had not been exposed to hydrogen evolution and the area which had been exposed to hydrogen evolution. This was performed in the manner described previously (2.4.4). Volta potential drop is shown against the hydrogen evolution charging time in Figure 93.

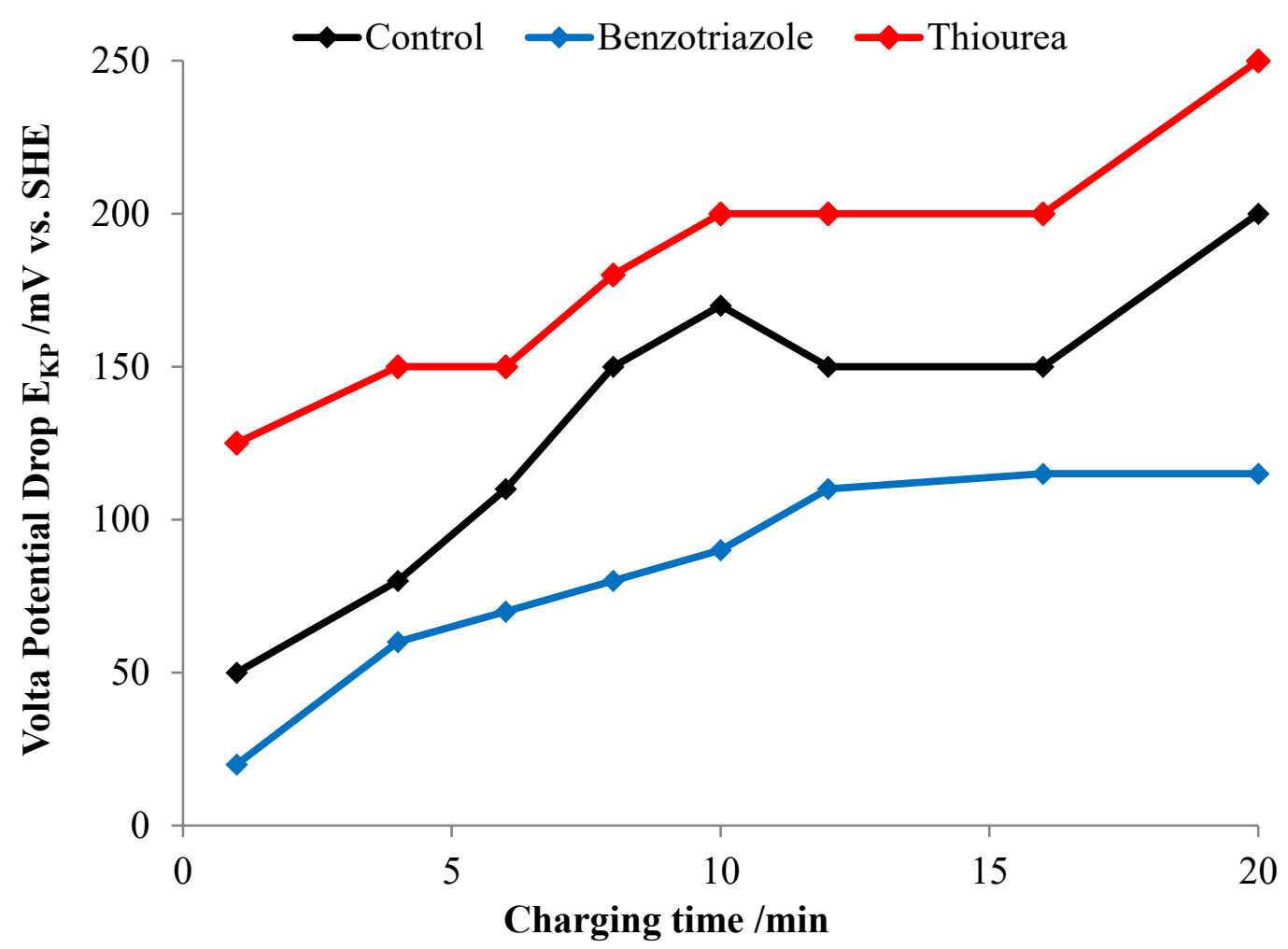

Figure 93 Volta potential drop vs. charging time resulting from hydrogen evolution on an iron susbstrate with a $0.2 \mathrm{M}$ potassium hydroxide electrolyte and addititions of benzotriazole and thiourea

In all experiments, evolution of hydrogen resulted in a depression in the Volta potential with respect to the bare metal substrate which had not evolved hydrogen. The magnitude of the Volta potential drop is different depending on both the charging time and the presence or absence of inhibitors.

Thiourea shows a greater depression in the Volta potential in the area which had been exposed to hydrogen evolution, compared to the equivalent area in the control samples. This suggests that thiourea encourages atomic hydrogen permeation in to the substrate. 
Conversely, benzotriazole additions result in a smaller depression in Volta potential when compared with the uninhibited control sample. This suggests that benzotriazole blocks hydrogen permeation into the substrate.

Results follow an approximate linear trend from a charge time of 1 minute up to 20 minutes. The control sample in particular shows a peak when the charging pre-treatment was for 8-10 minutes. This is thought to be within experimental error or caused by effects which are not understood.

In experiments where the charging time exceeded 20 minutes, the Volta potential drop did not continue to increase, as had generally been observed in charging times below 20 minutes. In this region where Volta potential drop was independent of charging time, it was considered to be due to saturation of the charging area with atomic hydrogen. However, when the sample was charged in the presence of benzotriazole, the Volta potential depression was $49 \%$ of the control sample. It is possible that benzotriazole limited the amount of hydrogen that could enter the sample, thus meaning that saturation was not reached or that a steady state was reached with hydrogen entering and leaving the charging area.

\subsection{Conclusions}

Prior to this study, a mechanism for the action of thiourea and benzotriazole was proposed but unverified and the SKP had not been used in energy storage studies. Initially, the inhibitors were ranked using the newly expanded Scanning Vibrating Electrode Technique, in the same manner as in the previous chapter. It was shown that additions of benzotriazole and thiourea to a potassium hydroxide electrolyte both result in a decrease in the amount of hydrogen evolved. Thiourea achieved the largest reduction in the hydrogen evolution rate of $92 \%$ vs. the control sample, whereas benzotriazole produced a rate reduction of a $66 \%$ vs. the control sample.

In line with the thesis aims, analysis of the mechanism of inhibition of benzotriazole and thiourea was performed using the SKP. Following a 'charging' pre-treatment where hydrogen was evolved from the sample, the SKP was used to scan the reverse face across the area where hydrogen had previously been evolved. A Volta potential drop was 
observed between the hydrogen evolution area and the area of the sample and the control area where no hydrogen had evolved. Literature reported that Volta potential difference observed was the effect of diffusing atomic hydrogen on the oxide layer through the sample surface. The Volta potential drop varied with respect to the duration of the charging pre-treatment and also with respect to the inhibitors used. The Volta potential drop was largest with thiourea and smallest with benzotriazole, with the uninhibited control sample falling between the two.

Given that thiourea was shown to be a more effective inhibitor for hydrogen evolution in the SVET experimentation, it could be expected that it would show the lowest Volta potential drop from the three samples. This assumption would be on the basis that if less hydrogen had evolved, there would be less atomic hydrogen present in the sample and, accordingly, less of an effect on the measured Volta potential (less of a Volta potential drop). Consistently in experimentation across the varying duration of charging pre-treatments, a greater potential drop was observed. This suggests a larger impact to the surface $\mathrm{Fe}^{\mathrm{II}}-\mathrm{Fe}^{\mathrm{III}}$ oxide layer, which is counterintuitive as the thiourea is more effective at halting hydrogen evolution. From this observation it is deduced that thiourea acts to block or slow the recombination reaction and thus does not stop the penetration of atomic hydrogen into the metal substrate.

Conversely, whilst benzotriazole is a less effective inhibitor than thiourea (measured using SVET), it still results in a significant reduction in hydrogen evolution when compared to the control sample with no inhibitor. As would be expected, a smaller Volta potential drop is observed between the benzotriazole tests than the control sample, across the range of the pre-treatment duration studied. Accordingly, it is determined that benzotriazole acts to inhibit the Volmer reaction, which in turn, inhibits the penetration of hydrogen into the metal substrate.

To conclude, whilst both additives act to inhibit the hydrogen evolution reaction, the mechanisms are different. Thiourea slows the recombination reaction, essential to hydrogen evolution, but as atomic hydrogen is already present in the electrode, it does not stop the penetration of atomic hydrogen into the metal substrate. This results in a larger amount of atomic hydrogen being present in the iron foil when thiourea is used as 
an inhibitor, compared to when no inhibitor is used (control) or when benzotriazole is used as an inhibitor. Benzotriazole inhibits a prior step in hydrogen evolution, the Volmer reaction and as a result there is less atomic hydrogen present in the bulk. Both effects are evidenced through the impact of atomic hydrogen on the surface $\mathrm{Fe}^{\mathrm{II}}-\mathrm{Fe}^{\mathrm{III}}$ oxide layer and subsequently, the local Volta potential differences that are observed.

Results from this study, using the SKP, are in agreement with those which were obtained by Amokrane et al using the Devanathan-Stachurski electrochemical double cell.

In addition to the agreement between results discussed by Amokrane et al, this experimentation also further confirms the ability for SKP to be used in hydrogen evolution studies.

Understanding the mechanism of inhibition of these inhibitors could have important implications for energy storage.

It is possible that a less effective inhibitor, such as benzotriazole, may be preferred if does not encourage atomic hydrogen to be present. This is because ferrous materials are sensitive to hydrogen embrittlement due to the presence of atomic hydrogen. Whilst some inhibitors may be less effective in an electrochemical sense, they may have advantages in the propensity to encourage hydrogen embrittlement.

\subsection{Further work}

Studies using the Kelvin probe have shown that palladium can be used to obtain quantitative measurements of mobile atomic hydrogen [176]. Evers and Rohwerder used physical vapor deposition (PVD) to plate a $25 \mu \mathrm{m}$ layer of palladium on to an iron membrane. In an extension of work documented in this Chapter, palladium plating could be employed to obtain quantitative measurements of hydrogen diffusion due to the electrode potential of palladium varying with respect to its atomic hydrogen concentration. In fact, this author attempted preliminary work where a layer of palladium of undetermined thickness was deposited using PVD. In results obtained, repeatability was not deemed sufficient to continue this work and this was not progressed further due 
to this researcher not having access to equipment which could be used to assess the thickness of the PVD palladium layer.

Further improvements in SKP experimentation could also be achieved by development of a dedicated cell which would allow access by the Scanning Kelvin Probe with simultaneous hydrogen evolution by cathodic polarization. Ability to commence and halt polarization, without alterations to experimental setup, would enhance the range of tests available to the researcher and further improve the understanding of the saturation point for atomic hydrogen. Additionally, it would be possible to add inhibitors to the electrolyte during experimentation to observe their effect and better understand their mechanism.

As noted by Evers et al, hydrogen has long been associated with embrittlement in ferrous metals [175]. Masuda performed a study using Kelvin Force Microscopy (KFM) to explore crack growth as a result of hydrogen embrittlement [219]. If an iron electrode is used in an in-service battery and there is some level of hydrogen evolution, hydrogen embrittlement could be exposed as a potential failure mechanism. An in depth understanding of hydrogen evolution and crack propagation in iron electrodes could be gained using the above-described methodology which could result in expansions to cell lifetime and cycle life.

Heterogeneity of electrodes could also be studied using SKP, in a similar manner to experimentation performed in Chapter 3. A height scan could be utilized to explore the effects of surface contours, in an experiment where SKP is used to assess the effect of hydrogen on the metal surface oxide layer.

Storage of hydrogen in metals in the form of metal hydrides is consistently of interest in the field of energy storage [175]. Further development of this technique may result in future research applications in this area. 


\section{Chapter 7: Conclusions and Further Work}

\subsection{Conclusions}

The below conclusions are drawn from the work described in this thesis:

- Rapid manufacture of iron electrodes from PTFE containing ink was attempted using near infrared (NIR) heating. Adhesion between the coating and various substrates was insufficient and electrodes delaminated during the volume expansion which occurred during charging and discharging.

- Sintered electrodes were rapidly manufactured in approximately 5 minutes using an infrared furnace. These electrodes were cycled successfully with a typical iron electrode voltage profile.

- Whilst a range of substrates were used, significant variation in results meant it was not possible to discern the effect of the substrate surface modification.

- The Scanning Vibrating Electrode Technique (SVET) was developed through the design and manufacture of sample holders and related equipment such that could be applied for the first time for an investigation in energy storage.

- SVET was successfully verified against a traditional gas collection technique for in situ study of hydrogen evolution and used to rank inhibitors with the advantage of mapping activity across the sample surface.

- It was further demonstrated that the SVET could be utilized on rough sintered electrodes by assessment of sintered electrodes which were manufactured in earlier work.

- The Scanning Kelvin Probe (SKP) was used to study the mechanism and rank the performance of hydrogen inhibitors thiourea and benzotriazole through their effect on the levels of atomic hydrogen in an iron electrode.

- SKP findings agreed with results obtained through use of a Devanathan-Stachurski cell reported by other researchers, with the additional advantage of spatial resolution. 
- It was determined that thiourea, may not perform as well in long term cycling as benzotriazole, due to its effect of significantly increasing the amount of atomic hydrogen present in the electrode which may result in hydrogen embrittlement.

\subsection{Further Work}

In order to progress research in this field, further work is recommended on the themes of research discussed in this thesis. For low temperature iron electrode manufacture:

- Due to the previously discussed failures in adhesion of low temperature rapid manufactured iron electrodes, further work in this area should focus on efforts to improve the adhesion to the substrate. In 3.6, use of a flux is suggested, however a goal of this work should be benchmarking performance against similar systems reported in literature.

- Other approaches to low temperature iron electrodes could involve changes to the ink formulation to better suit rapid heating by reducing the content of volatile organic compounds (VOCs). Whilst this may prevent screen printing, if it enables successful rapid manufacture it may allow for layered electrodes to be developed which could offer simultaneous improvements to capacity and power capability (3.6).

To improve on the sintered iron electrodes discussed in Chapter 4:

- Focus on modification of the ink formulation in order to enable increased heating rates without suffering solvent boil as discussed in 4.5.3.

- Inclusion of a reducing agent mixed into the ink could allow for sintering in air without suffering excessive oxidation, which would make the process more economically viable (4.2).

- As the furnace used in this work was limited (4.5.1), sintering speed advantages could be sought by using a more suitable near infrared oven.

- Blowing agents may allow for a lower density structure to be produced, or a layered structure with a graduated porosity, expanding on work in 4.8. 
To further the analytical electrochemical techniques (Chapter 5 and Chapter 6):

- Expand on the capability of the SVET and SKP by testing a wider range of materials and electrode types, with more complex geometries. This is considered a continuation of work discussed in 5.5.

- Electrode degradation could also be studied using these techniques, by repeatedly scanning the same surface over time, or by cycling of a functioning cell.

- Use palladium in SKP studies to obtain quantitative measurements of atomic hydrogen, as proposed in 6.5. This would expand upon the SKP work presented in Chapter 6, however would require dedicated physical vapor deposition equipment and related analytical equipment to assess the quality of the deposited layer.

- Interesting SKP results could be obtained if a researcher was able to develop a dedicated jig or cell which would allow for simultaneous scanning of an iron electrode and hydrogen evolution. As mentioned in 6.5, this would allow for additions of inhibitors mid-experiment, however this would be a significant undertaking!

Development of analytical techniques and advancement of iron electrode manufacturing are important steps in progressing this technology for utility scale energy storage. 


\section{References}

[1] Chase, Jenny, 'Breakthroughs in Solar Power - It's not just about technology any more', presented at the 2012 Bloomberg New Energy Finance Summit, 05-Apr2012.

[2] A. Louwen, W. van Sark, R. Schropp and A. Faaij, 'A cost roadmap for silicon heterojunction solar cells', Sol. Energy Mater. Sol. Cells, vol. 147, pp. 295-314, Apr. 2016.

[3] Matthew Strong, 'Germany sets renewable energy record', Taiwan news, 09Jan-2018.

[4] 'RE to Meet 36\% of Germany's Electricity Consumption in 2017 | Trends | Renewable Energy Institute', Renewable Energy Institute, 11-Jan-2018.

[5] BBC, 'UK "had greenest year for electricity", BBC News, 28-Dec-2017.

[6] Press Association, 'Renewables generated triple the power of coal in 2017, UK figures show', The Guardian, 28-Dec-2017.

[7] X. Luo, J. Wang, M. Dooner and J. Clarke, 'Overview of current development in electrical energy storage technologies and the application potential in power system operation', Appl. Energy, vol. 137, pp. 511-536, Jan. 2015.

[8] D. Connolly, H. Lund, B. V. Mathiesen and M. Leahy, 'A review of computer tools for analysing the integration of renewable energy into various energy systems', Appl. Energy, vol. 87, no. 4, pp. 1059-1082, Apr. 2010.

[9] T. Nakata, D. Silva and M. Rodionov, 'Application of energy system models for designing a low-carbon society’, Prog. Energy Combust. Sci., vol. 37, no. 4, pp. 462502, Aug. 2011.

[10] S. Bahramara, M. P. Moghaddam and M. R. Haghifam, 'Optimal planning of hybrid renewable energy systems using HOMER: A review', Renew. Sustain. Energy Rev., vol. 62, pp. 609-620, Sep. 2016.

[11] A. Chauhan and R. P. Saini, 'A review on Integrated Renewable Energy System based power generation for stand-alone applications: Configurations, storage options, sizing methodologies and control', Renew. Sustain. Energy Rev., vol. 38, pp. 99-120, Oct. 2014.

[12] Liam Stoker, 'Solar central to Sainsbury's sustainability plan as supermarket trials battery storage', Solar Power Portal.

[13] Russell Lyne, 'Marks \& Spencer crowdfunds solar panels for its stores', $U K$ Solar Generation, 21-Jun-2016.

[14] 'Total number of households by region and country of the UK, 1996 to 2016 Office for National Statistics'. [Online]. Available:

https://www.ons.gov.uk/peoplepopulationandcommunity/birthsdeathsandmarriages/fa milies/adhocs/005374totalnumberofhouseholdsbyregionandcountryoftheuk1996to201 5. [Accessed: 21-Jan-2018].

[15] Chris Wright, 'Storage is accelerating the low-carbon energy revolution', Environment Journal, 12-Oct-2016. 
[16] M. Zheng, C. J. Meinrenken and K. S. Lackner, 'Smart households: Dispatch strategies and economic analysis of distributed energy storage for residential peak shaving', Appl. Energy, vol. 147, pp. 246-257, Jun. 2015.

[17] Hugo Griffiths, 'Nissan sells solar panels and storage batteries to homeowners', Auto Express, 19-Jan-2018.

[18] J. O. G. Posada et al., 'Aqueous batteries as grid scale energy storage solutions', Renew. Sustain. Energy Rev., vol. 68, pp. 1174-1182, Feb. 2017.

[19] C. Yang, A. K. Manohar and S. R. Narayanan, 'A High-Performance Sintered Iron Electrode for Rechargeable Alkaline Batteries to Enable Large-Scale Energy Storage', J. Electrochem. Soc., vol. 164, no. 2, pp. A418-A429, Jan. 2017.

[20] H. Wang et al., 'An ultrafast nickel-iron battery from strongly coupled inorganic nanoparticle/nanocarbon hybrid materials', Nat. Commun., vol. 3, p. 917, Jun. 2012.

[21] G. P. Hammond and T. Hazeldine, 'Indicative energy technology assessment of advanced rechargeable batteries', Appl. Energy, vol. 138, no. Supplement C, pp. 559571, Jan. 2015.

[22] A. K. Manohar, S. Malkhandi, B. Yang, C. Yang, G. K. S. Prakash and S. R. Narayanan, 'A High-Performance Rechargeable Iron Electrode for Large-Scale Battery-Based Energy Storage', J. Electrochem. Soc., vol. 159, no. 8, pp. A1209A1214, Jan. 2012.

[23] Y. V. Makarov et al., 'Wide-Area Energy Storage and Management System to Balance Intermittent Resources in the Bonneville Power Administration and California ISO Control Areas'. Pacific Northwest National Laboratory on behalf of the U.S Department of Energy, Jun-2008.

[24] 'BioSolar's says biobased Super Battery tech can crush energy storage costs : Biofuels Digest'. .

[25] 'Feature: Power shift', Cosmos Magazine. [Online]. Available: https://cosmosmagazine.com/technology/power-shift. [Accessed: 31-Mar-2019].

[26] T. Reddy and D. Linden, Linden's Handbook of Batteries, 4th ed. McGraw-Hill Professional, 2010.

[27] Gates Energy Products, Rechargeable Batteries Applications Handbook. Elsevier, 1998.

[28] A. S. Rajan, S. Sampath and A. K. Shukla, 'An in situ carbon-grafted alkaline iron electrode for iron-based accumulators', Energy Environ. Sci., vol. 7, no. 3, pp. 1110-1116, Feb. 2014.

[29] H. A. Figueredo-Rodríguez et al., 'A Rechargeable, Aqueous Iron Air Battery with Nanostructured Electrodes Capable of High Energy Density Operation', $J$. Electrochem. Soc., vol. 164, no. 6, pp. A1148-A1157, Jan. 2017.

[30] J. H. Pikul, H. Gang Zhang, J. Cho, P. V. Braun and W. P. King, 'High-power lithium ion microbatteries from interdigitated three-dimensional bicontinuous nanoporous electrodes', Nat. Commun., vol. 4, p. 1732, Apr. 2013.

[31] MIT Electric Vehicle Team, 'A Guide to Understanding Battery Specifications'. Massachusetts Institute of Technology, Dec-2008. 
[32] G. Halpert, 'Past developments and the future of nickel electrode cell technology', J. Power Sources, vol. 12, no. 3-4, pp. 177-192, Jul. 1984.

[33] Directive of the European Parliment, 'Directive 2006/66/EC of the European Parliment and of the Council of 6 September 2006 on batteries and accumulators and waste batteries and accumulators and repealing Directive 91/157/EEC'.

[34] Claus Daniel and J. O. Besenhard, Handbook of Battery Materials, 2nd ed. Wiley, 2011.

[35] K. Desmond, Innovators in Battery Technology: Profiles of 95 Influential Electrochemists. McFarland, 2016.

[36] A. J. Salkind, D. T. Ferrel and A. J. Hedges, 'Secondary batteries - 1952-1977', J. Electrochem. Soc., vol. 125, p. 311, Aug. 1978.

[37] I. Mabbett, J. Malone and D. A. Worsley, 'Comparison of Pocket Plate and Sintered Plate Ni Electrodes Used in NiFe Cells for PV Back up Applications', Meet. Abstr., vol. MA2012-02, no. 5, pp. 368-368, Jun. 2012.

[38] Hideo Takeshita, 'Proceedings of the 27th international battery seminar', Fort Lauderdale, FL, USA, Mar-2010.

[39] Bloomberg New Energy Finance, 'Lithium ion battery costs and market', 05-Jul2017.

[40] J. Whitacre, 'Aqueous Electrolyte Polyionic Batteries for Scaled Stationary Energy Storage', Meet. Abstr., vol. MA2016-02, no. 1, pp. 132-132, Sep. 2016.

[41] 'Deep Cycle Batteries | Deep Cycle Battery Solar | Aquion Energy', Aquion. [Online]. Available: http://aquionenergy.com/technology/deep-cycle-battery/. [Accessed: 06-Jan-2018].

[42] Y. Li et al., 'Recent advances of electrode materials for low-cost sodium-ion batteries towards practical application for grid energy storage', Energy Storage Mater., vol. 7, no. Supplement C, pp. 130-151, Apr. 2017.

[43] M. W. Orzech et al., 'Synergic effect of Bi, Sb and Te for the increased stability of bulk alloying anodes for sodium-ion batteries', J. Mater. Chem. A, vol. 5, no. 44, pp. 23198-23208, Nov. 2017.

[44] L. Duchêne et al., 'A stable 3 V all-solid-state sodium-ion battery based on a closo-borate electrolyte', Energy Environ. Sci., vol. 10, no. 12, pp. 2609-2615, Dec. 2017.

[45] W. Zhou, Y. Li, S. Xin and J. B. Goodenough, 'Rechargeable Sodium All-SolidState Battery', ACS Cent. Sci., vol. 3, no. 1, pp. 52-57, Jan. 2017.

[46] 'Lithium-Ion Pioneer Introduces New Battery That's Three Times Better', Fortune. [Online]. Available: http://fortune.com/2017/03/05/lithium-ion-batterygoodenough/. [Accessed: 06-Jan-2018].

[47] J. Conca, 'The Beguiling Promise Of John Goodenough's New Battery Technology', Forbes. [Online]. Available:

https://www.forbes.com/sites/jamesconca/2017/03/17/jack-goodenoughs-batterytechnologies-keep-getting-better/. [Accessed: 06-Jan-2018]. 
[48] M. H. Braga, N. S. Grundish, A. J. Murchison and J. B. Goodenough, 'Alternative strategy for a safe rechargeable battery', Energy Environ. Sci., vol. 10, no. 1, pp. 331-336, Jan. 2017.

[49] 'Goodenough Making Progress With Solid-State Batteries: Q\&A', Bloomberg New Energy Finance, 02-Nov-2017. [Online]. Available:

https://about.bnef.com/blog/goodenough-making-progress-solid-state-batteries-qa/. [Accessed: 06-Jan-2018].

[50] 'Fisker | News'. [Online]. Available: https://www.fiskerinc.com/news. [Accessed: 06-Jan-2018].

[51] F. Lambert, 'Fisker claims solid-state battery "breakthrough" for electric cars with "500 miles range and 1 min charging", Electrek, 14-Nov-2017. .

[52] S. R. Narayanan, G. K. S. Prakash, A. K. Manohar, B. Yang, S. Malkhandi and A. Kindler, 'Materials challenges and technical approaches for realizing inexpensive and robust iron-air batteries for large-scale energy storage', Solid State Ion., vol. 216, pp. 105-109, May 2012.

[53] B. Andersson and L. Ojefors, 'Optimization of Iron-Air and Nickel Oxide-Iron Traction Batteries', Dechema Monogr. Dtsch. Ges. Fuer Chem. Apparatewesen, pp. 329-343, 1979.

[54] J. O. Gil Posada and P. J. Hall, 'Multivariate investigation of parameters in the development and improvement of NiFe cells', J. Power Sources, vol. 262, pp. $263-$ 269, Sep. 2014.

[55] 'Changhong Nickel-lron (Ni-Fe) Batteries', Changhong Batteries. [Online]. Available: http://www.changhongbatteries.com/Nickel-lron(Ni-Fe)_c13_m2.2.1.html. [Accessed: 23-May-2013].

[56] S. U. Falk and A. J. Salkind, Alkaline storage batteries. Wiley, 1969.

[57] A. J. Salkind, 'Storage battery electrodes and methods for making them', US3062908 A, 06-Nov-1962.

[58] M. J. M. Jr and A. J. Salkind, 'Gas depressor additives for iron electrodes', US3484291 A, 16-Dec-1969.

[59] A. J. Salkind, C. J. Venuto and S. U. Falk, 'The Reaction at the Iron Alkaline Electrode', J. Electrochem. Soc., vol. 111, no. 5, pp. 493-495, May 1964.

[60] A. J. Salkind and M. Klein, 'Batteries, Alkaline Secondary Cells', in KirkOthmer Encyclopedia of Chemical Technology, John Wiley \& Sons, Inc., 2000.

[61] L. Öjefors, 'SEM Studies of Discharge Products from Alkaline Iron Electrodes', J. Electrochem. Soc., vol. 123, no. 11, pp. 1691-1696, Nov. 1976.

[62] L. Öjefors, 'Self-discharge of the alkaline iron electrode', Electrochimica Acta, vol. 21, no. 4, pp. 263-266, Apr. 1976.

[63] E. S. Buzzelli, C. T. Liu and W. A. Bryant, 'Iron-air batteries for electric vehicles', presented at the 13th Intersociety Energy Conversion Engineering Conference, 1978, pp. 745-749.

[64] W. A. Bryant, 'The Importance of Physical Structure to the Capacity of Porous Iron Electrodes', J. Electrochem. Soc., vol. 126, no. 11, pp. 1899-1901, Nov. 1979. 
[65] W. A. Bryant, 'The structure and performance of sintered iron electrodes', Electrochimica Acta, vol. 24, no. 10, pp. 1057-1060, Oct. 1979.

[66] B. Hariprakash, S. K. Martha, M. S. Hegde and A. K. Shukla, 'A sealed, starved-electrolyte nickel-iron battery', J. Appl. Electrochem., vol. 35, no. 1, pp. 2732, Jan. 2005.

[67] T. S. Balasubramanian and A. K. Shukla, 'Effect of metal-sulfide additives on charge/discharge reactions of the alkaline iron electrode', J. Power Sources, vol. 41, no. 1-2, pp. 99-105, Jan. 1993.

[68] A. K. Shukla, M. K. Ravikumar and T. S. Balasubramanian, 'Nickel/iron batteries', J. Power Sources, vol. 51, no. 1-2, pp. 29-36, Aug. 1994.

[69] T. S. Balasubramanian, K. Vijayamohanan and A. K. Shukla, 'Mechanisms of the discharge of porous-iron electrodes in alkaline medium', J. Appl. Electrochem., vol. 23, no. 9, pp. 947-950, Sep. 1993.

[70] M. K. Ravikumar, T. S. Balasubramanian and A. K. Shukla, 'A nickel-iron battery with roll-compacted iron electrodes', J. Power Sources, vol. 56, no. 2, pp. 209-212, Aug. 1995.

[71] S. A. Gaffor, B. Haripraksh and A. K. shukla, 'Nickel-iron battery-based electrochemical energy storage systems for rural/remote area telecommunication', in Intelec 2010, 2010, pp. 1-6.

[72] K. Vijayamohanan, A. K. Shukla and S. Sathyanarayana, 'Kinetics of electrode reactions occurring on porous iron electrodes in alkaline media', J. Electroanal. Chem. Interfacial Electrochem., vol. 295, no. 1, pp. 59-70, Nov. 1990.

[73] K. Vijayamohanan, A. K. Shukla and S. Sathyanarayana, 'Open-circuit potential-recovery transients of alkaline porous iron electrodes', J. Power Sources, vol. 21, no. 1, pp. 53-57, Aug. 1987.

[74] M. K. Ravikumar, A. S. Rajan, S. Sampath, K. R. Priolkar and A. K. Shukla, 'In Situ Crystallographic Probing on Ameliorating Effect of Sulfide Additives and Carbon Grafting in Iron Electrodes', J. Electrochem. Soc., vol. 162, no. 12, pp. A2339-A2350, Jan. 2015.

[75] P. Periasamy, B. R. Babu and S. V. Iyer, 'Cyclic voltammetric studies of porous iron electrodes in alkaline solutions used for alkaline batteries', J. Power Sources, vol. 58, no. 1, pp. 35-40, Jan. 1996.

[76] P. Periasamy, B. Ramesh Babu and S. Venkatakrishna Iyer, 'Performance characterization of sintered iron electrodes in nickel/iron alkaline batteries', J. Power Sources, vol. 62, no. 1, pp. 9-14, Sep. 1996.

[77] P. Periasamy, B. Ramesh Babu and S. Venkatakrishna Iyer, 'Electrochemical behaviour of Teflon-bonded iron oxide electrodes in alkaline solutions', J. Power Sources, vol. 63, no. 1, pp. 79-85, Nov. 1996.

[78] C. Chakkaravarthy, P. Periasamy, S. Jegannathan and K. I. Vasu, 'The nickel/iron battery', J. Power Sources, vol. 35, no. 1, pp. 21-35, Jun. 1991.

[79] S. Malkhandi, B. Yang, A. K. Manohar, G. K. S. Prakash and S. R. Narayanan, 'Self-Assembled Monolayers of n-Alkanethiols Suppress Hydrogen Evolution and 
Increase the Efficiency of Rechargeable Iron Battery Electrodes', J. Am. Chem. Soc., vol. 135, no. 1, pp. 347-353, Jan. 2013.

[80] A. K. Manohar, C. Yang, S. Malkhandi, B. Yang, G. K. S. Prakash and S. R. Narayanan, 'Understanding the Factors Affecting the Formation of Carbonyl Iron Electrodes in Rechargeable Alkaline Iron Batteries', J. Electrochem. Soc., vol. 159, no. 12, pp. A2148-A2155, Jan. 2012.

[81] a. K. Manohar, C. Yang, S. Malkhandi, G. K. S. Prakash and S. R. Narayanan, 'Enhancing the Performance of the Rechargeable Iron Electrode in Alkaline Batteries with Bismuth Oxide and Iron Sulfide Additives', J. Electrochem. Soc., vol. 160, no. 11, pp. A2078-A2084, Oct. 2013.

[82] B. Yang, S. Malkhandi, A. K. Manohar, G. K. S. Prakash and S. R. Narayanan, 'Organo-sulfur molecules enable iron-based battery electrodes to meet the challenges of large-scale electrical energy storage', Energy Environ. Sci., vol. 7, no. 8, pp. 27532763, Jul. 2014.

[83] A. K. Manohar, C. Yang and S. R. Narayanan, 'The Role of Sulfide Additives in Achieving Long Cycle Life Rechargeable Iron Electrodes in Alkaline Batteries', $J$. Electrochem. Soc., vol. 162, no. 9, pp. A1864-A1872, Jan. 2015.

[84] S. Narayanan, 'Performance of Sintered Iron Electrodes for Rechargeable Alkaline Batteries for Large Scale Energy Storage', presented at the 225th ECS Meeting (May 11-15, 2014), 2014.

[85] S. Malkhandi et al., 'Electrocatalytic Activity of Transition Metal Oxide-Carbon Composites for Oxygen Reduction in Alkaline Batteries and Fuel Cells', $J$. Electrochem. Soc., vol. 160, no. 9, pp. F943-F952, Jan. 2013.

[86] J. O. G. Posada and P. J. Hall, 'Towards the development of safe and commercially viable nickel-iron batteries: improvements to Coulombic efficiency at high iron sulphide electrode formulations', J. Appl. Electrochem., vol. 46, no. 4, pp. 451-458, Apr. 2016.

[87] J. O. G. Posada and P. J. Hall, 'Controlling hydrogen evolution on iron electrodes', Int. J. Hydrog. Energy, vol. 41, no. 45, pp. 20807-20817, Dec. 2016.

[88] J. O. Gil Posada, A. H. Abdalla, C. I. Oseghale and P. J. Hall, 'Multiple regression analysis in the development of $\mathrm{NiFe}$ cells as energy storage solutions for intermittent power sources such as wind or solar', Int. J. Hydrog. Energy, vol. 41, no. 37, pp. 16330-16337, Oct. 2016.

[89] A. Abdalla, C. Oseghale, J. Gil-Posada and P. Hall, 'Rechargeable Nickel-Iron Batteries for large-scale Energy storage', IET Renew. Power Gener., Sep. 2016.

[90] J. O. G. Posada and P. J. Hall, 'The Effect of Electrolyte Additives on the Performance of Iron Based Anodes for NiFe Cells', J. Electrochem. Soc., vol. 162, no. 10, pp. A2036-A2043, Jan. 2015.

[91] J. O. G. Posada and P. J. Hall, 'Surface response investigation of parameters in the development of FeS based iron electrodes', Sustain. Energy Technol. Assess., vol. 11, Sep. 2015.

[92] R. Ogg, 'Battery comprising a coated iron anode', US20140220430 A1, 07 Aug-2014. 
[93] R. G. Ogg, 'Coated iron electrode and method of making same', US20140220440 A1, 07-Aug-2014.

[94] R. Ogg, 'Nickel-iron battery with high cycle life', US20140220431 A1, 07-Aug2014.

[95] R. G. Ogg and A. P. Seidel, 'Nickel iron battery employing an untreated polyolefin separator with a surfactant in the electrolyte', US20160372794 A1, 22Dec-2016.

[96] R. G. Ogg and C. H. Welch, 'Layered iron electrode', US20140220433 A1, $07-$ Aug-2014.

[97] R. Ogg, C. H. Welch and A. P. Seidel, 'Iron electrode employing a polyvinyl alcohol binder', WO2014121013 A1, 07-Aug-2014.

[98] R. G. Ogg, M. Roders and M. Messe, 'Process of preparing a chemically preformed (CPF) iron negative electrode with water', US9816170 B2, 14-Nov-2017.

[99] R. G. Ogg, C. Welch and A. Seidel, 'Iron electrode employing a polyvinyl alcohol binder', US9478806 B2, 25-Oct-2016.

[100] R. Bonnaterre, R. Doisneau, M. C. Petit and J. P. Stervinou, 'Power sources 7: Research and development in non-mechanical electrical power sources', in Eleventh International Symposium, Journal of Power Sources, September 25-28 1978, Brighton, Sussex, England, 1979, vol. 7, p. 249.

[101] L. Öjefors and L. Carlsson, 'An iron-air vehicle battery', J. Power Sources, vol. 2, no. 3, pp. 287-296, Feb. 1978.

[102] J. Liu et al., 'A Flexible Alkaline Rechargeable Ni/Fe Battery Based on Graphene Foam/Carbon Nanotubes Hybrid Film', Nano Lett., vol. 14, no. 12, pp. 7180-7187, Dec. 2014.

[103] W. Jiang, F. Liang, J. Wang, L. Su, Y. Wu and L. Wang, 'Enhanced electrochemical performances of $\mathrm{FeOx}$-graphene nanocomposites as anode materials for alkaline nickel-iron batteries', $R S C A d v$., vol. 4, no. 30, pp. 15394-15399, Mar. 2014

[104] D. Lei, D.-C. Lee, A. Magasinski, E. Zhao, D. Steingart and G. Yushin, 'Performance Enhancement and Side Reactions in Rechargeable Nickel-Iron Batteries with Nanostructured Electrodes', ACS Appl. Mater. Interfaces, vol. 8, no. 3, pp. 2088-2096, Jan. 2016.

[105] C. Huang, N. P. Young, J. Zhang, H. J. Snaith and P. S. Grant, 'A two layer electrode structure for improved Li Ion diffusion and volumetric capacity in Li Ion batteries', Nano Energy, vol. 31, pp. 377-385, Jan. 2017.

[106] C. Huang et al., 'Layer-by-layer spray deposition and unzipping of single-wall carbon nanotube-based thin film electrodes for electrochemical capacitors', Carbon, vol. 61, pp. 525-536, Sep. 2013.

[107] V. I. Chani, Q. Yang, D. S. Wilkinson and G. C. Weatherly, 'Effect of substrate pre-coating on adhesion of sintered nickel plaques for electrode application in rechargeable batteries', J. Power Sources, vol. 142, no. 1-2, pp. 370-381, Mar. 2005. 
[108] M. R. Gennero de Chialvo and A. C. Chialvo, 'Hydrogen evolution reaction on a smooth iron electrode in alkaline solution at different temperatures', Phys. Chem. Chem. Phys., vol. 3, no. 15, pp. 3180-3184, Jan. 2001.

[109] M. R. Gennero de Chialvo and A. C. Chialvo, 'Hydrogen evolution reaction: Analysis of the Volmer-Heyrovsky-Tafel mechanism with a generalized adsorption model', J. Electroanal. Chem., vol. 372, no. 1, pp. 209-223, Jul. 1994.

[110] C. G. Morales-Guio, L.-A. Stern and X. Hu, 'Nanostructured hydrotreating catalysts for electrochemical hydrogen evolution', Chem. Soc. Rev., vol. 43, no. 18, pp. 6555-6569, Aug. 2014.

[111] A. K. Shukla, S. Venugopalan and B. Hariprakash, 'Nickel-based rechargeable batteries', J. Power Sources, vol. 100, no. 1-2, pp. 125-148, Nov. 2001.

[112] J. O. Gil Posada and P. J. Hall, 'Post-hoc comparisons among iron electrode formulations based on bismuth, bismuth sulphide, iron sulphide and potassium sulphide under strong alkaline conditions', J. Power Sources, vol. 268, no. Supplement C, pp. 810-815, Dec. 2014.

[113] C. A. C. Souza, I. A. Carlos, M. Lopes, G. A. Finazzi and M. R. H. de Almeida, 'Self-discharge of Fe-Ni alkaline batteries', J. Power Sources, vol. 132, no. 1-2, pp. 288-290, May 2004.

[114] C.-Y. Kao and K.-S. Chou, 'Iron/carbon-black composite nanoparticles as an iron electrode material in a paste type rechargeable alkaline battery', J. Power Sources, vol. 195, no. 8, pp. 2399-2404, Apr. 2010.

[115] W. He, H. Shao, Q. Chen, J. Wang and J. Zhang, 'Polarization Characteristic of Iron Anode in Concentrated NaOH Solution', Acta Phys.-Chim. Sin., vol. 23, no. 10, pp. 1525-1530, Oct. 2007.

[116] K.-C. Huang and K.-S. Chou, 'Microstructure changes to iron nanoparticles during discharge/charge cycles', Electrochem. Commun., vol. 9, no. 8, pp. 19071912, Aug. 2007.

[117] C.-Y. Kao, Y.-R. Tsai and K.-S. Chou, 'Synthesis and characterization of the iron/copper composite as an electrode material for the rechargeable alkaline battery', J. Power Sources, vol. 196, no. 13, pp. 5746-5750, Jul. 2011.

[118] U. Casellato, N. Comisso and G. Mengoli, 'Effect of Li ions on reduction of Fe oxides in aqueous alkaline medium', Electrochimica Acta, vol. 51, no. 26, pp. 56695681, Aug. 2006.

[119] A. A. Kamnev, 'The role of lithium in preventing the detrimental effect of iron on alkaline battery nickel hydroxide electrode: A mechanistic aspect', Electrochimica Acta, vol. 41, no. 2, pp. 267-275, Feb. 1996.

[120] M. Jayalakshmi and V. S. Muralidharan, 'Influence of Sulfur on Passivation of Iron in Alkali Solutions', Corrosion, vol. 48, no. 11, pp. 918-923, Nov. 1992.

[121] V. I. Vigdorovich, L. E. Tsygankova, O. V. Alekhina and T. P. D’yachkova, 'Effect of Thiourea on the Kinetics of the Hydrogen Evolution Reaction at Iron and the Transport of Hydrogen through a Steel Membrane in Solutions of C2H4(OH)2H2O-HCl', Russ. J. Electrochem., vol. 41, no. 10, pp. 1046-1052, Oct. 2005. 
[122] J.-N. Han, S.-I. Pyun and T.-H. Yang, 'Roles of Thiourea as an Inhibitor in Hydrogen Absorption into Palladium Electrode', J. Electrochem. Soc., vol. 144, no. 12, pp. 4266-4272, Dec. 1997.

[123] N. Amokrane, C. Gabrielli, G. Maurin and L. Mirkova, 'Effect of organic additives on hydrogen permeation into an iron membrane studied by frequency analysis techniques', Electrochimica Acta, vol. 53, no. 4, pp. 1962-1971, Dec. 2007.

[124] A. Fateh, M. Aliofkhazraei and A. R. Rezvanian, 'Review of corrosive environments for copper and its corrosion inhibitors', Arab. J. Chem., Jun. 2017.

[125] D. Zhang, L. Gao and G. Zhou, 'Inhibition of copper corrosion in aerated hydrochloric acid solution by amino-acid compounds', J. Appl. Electrochem., vol. 35, no. 11, pp. 1081-1085, Nov. 2005.

[126] Y. H. Lei, N. Sheng, A. Hyono, M. Ueda and T. Ohtsuka, 'Effect of benzotriazole (BTA) addition on Polypyrrole film formation on copper and its corrosion protection', Prog. Org. Coat., vol. 77, no. 2, pp. 339-346, Feb. 2014.

[127] N. K. Allam, A. A. Nazeer and E. A. Ashour, 'A review of the effects of benzotriazole on the corrosion of copper and copper alloys in clean and polluted environments', J. Appl. Electrochem., vol. 39, no. 7, pp. 961-969, Jul. 2009.

[128] G. Williams, A. J. Coleman and H. N. McMurray, 'Inhibition of Aluminium Alloy AA2024-T3 pitting corrosion by copper complexing compounds', Electrochimica Acta, vol. 55, no. 20, pp. 5947-5958, Aug. 2010.

[129] N. Eldakar and K. Nobe, 'Effect of Substituted Benzotriazoles on the Anodic Dissolution of Iron in H2SO4', CORROSION, vol. 37, no. 5, pp. 271-278, May 1981.

[130] R. Alkire and A. Cangellari, 'Effect of Benzotriazole on the Anodic Dissolution of Iron in the Presence of Fluid Flow', J. Electrochem. Soc., vol. 135, no. 10, pp. 2441-2446, Oct. 1988.

[131] B. Sathianandhan, K. Balakrishnan and N. Subramanyan, 'Triazoles as inhibitors of corrosion of mild steel in acids', Br. Corros. J., vol. 5, no. 6, pp. 270-273, 1970.

[132] S. M. Abd El Haleem, S. Abd El Wanees and A. Bahgat, 'Environmental factors affecting the corrosion behaviour of reinforcing steel. VI. Benzotriazole and its derivatives as corrosion inhibitors of steel', Corros. Sci., vol. 87, no. Supplement C, pp. 321-333, Oct. 2014.

[133] P. F. Khan, V. Shanthi, R. K. Babu, S. Muralidharan and R. C. Barik, 'Effect of benzotriazole on corrosion inhibition of copper under flow conditions', J. Environ. Chem. Eng., vol. 3, no. 1, pp. 10-19, Mar. 2015.

[134] H. Kitamura, L. Zhao, B. T. Hang, S. Okada and J. Yamaki, 'Effect of binder materials on cycling performance of $\mathrm{Fe} 2 \mathrm{O} 3$ electrodes in alkaline solution', J. Power Sources, vol. 208, pp. 391-396, Jun. 2012.

[135] P. R. Vassie and A. C. C. Tseung, 'High performance, rechargeable sintered iron electrodes - I: The effect of preparative methods and additives on the structure and performance of sintered iron electrodes', Electrochimica Acta, vol. 21, no. 4, pp. 299-302, Apr. 1976. 
[136] E. D. Bendereva and S. T. Vylkanov, 'Activating effect of boron microadditions on sintering of powder alloy based on iron', Metallurgist, vol. 55, no. 9-10, pp. 761768, Jan. 2012.

[137] V. V. Korol'kov and B. Kibak, 'Sintering mechanism of iron powder with microadditions of boron', Powder Metall. Met. Ceram., vol. 36, no. 9-10, pp. 470473, Sep. 1997.

[138] I. Mabbett et al., 'Rapid Processing and Scanning Electrochemical Techniques Applied to Sintered Nickel Electrodes of Varying Thickness and Rate Capability', ECS Trans., vol. 64, no. 18, pp. 1-12, Feb. 2015.

[139] J. Marinaccio, I. Mabbett and C. F. Glover, 'Rapid Processing Techniques Applied to Sintered Nickel Battery Technologies for Utility Scale Applications', Meet. Abstr., vol. MA2015-03, no. 2, pp. 451-451, Jul. 2015.

[140] K. Hooper, M. Carnie, C. Charbonneau and T. Watson, 'Near Infrared Radiation as a Rapid Heating Technique for TiO2 Films on Glass Mounted Dye-Sensitized Solar Cells', Int. J. Photoenergy, vol. 2014, p. e953623, Nov. 2014.

[141] M. Cherrington, T. C. Claypole, D. Deganello, I. Mabbett, T. Watson and D. Worsley, 'Ultrafast near-infrared sintering of a slot-die coated nano-silver conducting ink', J. Mater. Chem., vol. 21, no. 21, pp. 7562-7564, May 2011.

[142] T. Watson, I. Mabbett, H. Wang, L. Peter and D. Worsley, 'Ultrafast near infrared sintering of $\mathrm{TiO} 2$ layers on metal substrates for dye-sensitized solar cells', Prog. Photovolt. Res. Appl., vol. 19, no. 4, pp. 482-486, 2011.

[143] D. A. Worsley, A. Belghazi and S. M. Powell, 'Study of corrosion mechanism occurring at cut edges of organically coated galvanised steels', Ironmak. Steelmak., vol. 26, no. 5, pp. 387-392, Oct. 1999.

[144] D. A. Worsley, H. N. McMurray and A. Belghazi, 'Determination of localised corrosion mechanisms using a scanning vibrating reference electrode technique', Chem. Commun., no. 24, pp. 2369-2370, Jan. 1997.

[145] H. N. McMurray, S. M. Powell and D. A. Worsley, 'Mechanistic changes in cut edge corrosion induced by variation of inhibitor pigmentation in organically coated galvanised steel', Br. Corros. J., vol. 36, no. 1, pp. 42-48, Jan. 2001.

[146] J. Elvins, J. A. Spittle, J. H. Sullivan and D. A. Worsley, 'The effect of magnesium additions on the microstructure and cut edge corrosion resistance of zinc aluminium alloy galvanised steel', Corros. Sci., vol. 50, no. 6, pp. 1650-1658, Jun. 2008 .

[147] D. J. Penney, J. H. Sullivan and D. A. Worsley, 'Investigation into the effects of metallic coating thickness on the corrosion properties of $\mathrm{Zn}-\mathrm{Al}$ alloy galvanising coatings', Corros. Sci., vol. 49, no. 3, pp. 1321-1339, Mar. 2007.

[148] S. M. Powell, H. N. McMurray and D. A. Worsley, 'Use of the Scanning Reference Electrode Technique for the Evaluation of Environmentally Friendly, Nonchromate Corrosion Inhibitors', Corrosion, vol. 55, no. 11, pp. 1040-1051, Nov. 1999. 
[149] H. N. McMurray and D. A. Worsley, 'Scanning Electrochemical Techniques for the Study of Localised Metallic Corrosion', in Advances in Chemical Kinetics, vol. 4, R. G. Compton and G. Hancock, Eds. Blackwell Science, Oxford, 1997, pp. 149-202.

[150] J. Elvins, J. A. Spittle and D. A. Worsley, 'Microstructural changes in zinc aluminium alloy galvanising as a function of processing parameters and their influence on corrosion', Corros. Sci., vol. 47, no. 11, pp. 2740-2759, Nov. 2005.

[151] S. Böhm, H. N. McMurray, S. M. Powell and D. A. Worsley, 'Photoelectrochemical investigation of corrosion using scanning electrochemical techniques', Electrochimica Acta, vol. 45, no. 14, pp. 2165-2174, Mar. 2000.

[152] J. Sullivan, C. Weirman, J. Kennedy and D. Penney, 'Influence of steel gauge on the microstructure and corrosion performance of zinc alloy coated steels', Corros. Sci., vol. 52, no. 5, pp. 1853-1862, May 2010.

[153] A. M. Simões and J. C. S. Fernandes, 'Studying phosphate corrosion inhibition at the cut edge of coil coated galvanized steel using the SVET and EIS', Prog. Org. Coat., vol. 69, no. 2, pp. 219-224, Oct. 2010.

[154] C. F. Glover, 'SVET Corrosion Image'.

[155] G. Williams and H. N. McMurray, 'Localized Corrosion of Magnesium in Chloride-Containing Electrolyte Studied by a Scanning Vibrating Electrode Technique', J. Electrochem. Soc., vol. 155, no. 7, pp. C340-C349, Jul. 2008.

[156] G. Williams, N. Birbilis and H. N. McMurray, 'The source of hydrogen evolved from a magnesium anode', Electrochem. Commun., vol. 36, pp. 1-5, Nov. 2013.

[157] G. Williams, H. Dafydd, H. N. McMurray and N. Birbilis, 'The influence of arsenic alloying on the localised corrosion behaviour of magnesium', Electrochimica Acta, vol. 219, pp. 401-411, Nov. 2016.

[158] S. Fajardo, C. f. Glover, G. Williams and G. s. Frankel, 'The Evolution of Anodic Hydrogen on High Purity Magnesium in Acidic Buffer Solution', CORROSION, vol. 73, no. 5, pp. 482-493, Jan. 2017.

[159] S. Fajardo, C. F. Glover, G. Williams and G. S. Frankel, 'The Source of Anodic Hydrogen Evolution on Ultra High Purity Magnesium', Electrochimica Acta, vol. 212, pp. 510-521, Sep. 2016.

[160] G. Williams and H. N. McMurray, 'Inhibition of corrosion driven delamination on iron by smart-release bentonite cation-exchange pigments studied using a scanning Kelvin probe technique', Prog. Org. Coat., vol. 102, pp. 18-28, Jan. 2017.

[161] H. N. McMurray, G. Williams and S. O'Driscoll, 'Chromate Inhibition of Filiform Corrosion on Organic Coated AA2024-T3 Studied Using the Scanning Kelvin Probe', J. Electrochem. Soc., vol. 151, no. 7, pp. B406-B414, Jul. 2004.

[162] G. Williams and R. Grace, 'Chloride-induced filiform corrosion of organiccoated magnesium', Electrochimica Acta, vol. 56, no. 4, pp. 1894-1903, Jan. 2011.

[163] G. Williams, H. N. McMurray and D. A. Worsley, 'Cerium(III) Inhibition of Corrosion-Driven Organic Coating Delamination Studied Using a Scanning Kelvin Probe Technique', J. Electrochem. Soc., vol. 149, no. 4, pp. B154-B162, Apr. 2002. 
[164] G. Williams and H. N. McMurray, 'Chromate Inhibition of Corrosion-Driven Organic Coating Delamination Studied Using a Scanning Kelvin Probe Technique', J. Electrochem. Soc., vol. 148, no. 10, pp. B377-B385, Oct. 2001.

[165] H. N. McMurray, A. J. Coleman, G. Williams, A. Afseth and G. M. Scamans, 'Scanning Kelvin Probe Studies of Filiform Corrosion on Automotive Aluminum Alloy AA6016', J. Electrochem. Soc., vol. 154, no. 7, pp. C339-C348, Jul. 2007.

[166] M. Stratmann, H. Streckel and R. Feser, 'A new technique able to measure directly the delamination of organic polymer films', Corros. Sci., vol. 32, no. 4, pp. 467-470, Jan. 1991.

[167] A. Q. Fu and Y. F. Cheng, 'Characterization of corrosion of X65 pipeline steel under disbonded coating by scanning Kelvin probe', Corros. Sci., vol. 51, no. 4, pp. 914-920, Apr. 2009.

[168] M. Stratmann, R. Feser and A. Leng, 'Corrosion protection by organic films', Electrochimica Acta, vol. 39, no. 8, pp. 1207-1214, Jun. 1994.

[169] B. Reddy and J. M. Sykes, 'Degradation of organic coatings in a corrosive environment: a study by scanning Kelvin probe and scanning acoustic microscope', Prog. Org. Coat., vol. 52, no. 4, pp. 280-287, Apr. 2005.

[170] G. Williams, H. N. McMurray and D. A. Worsley, 'Latent fingerprint detection using a scanning Kelvin microprobe’, J. Forensic Sci., vol. 46, no. 5, pp. 1085-1092, Sep. 2001.

[171] H. Dafydd, G. Williams and S. Bleay, 'Latent Fingerprint Visualization using a Scanning Kelvin Probe in Conjunction with Vacuum Metal Deposition', J. Forensic Sci., vol. 59, no. 1, pp. 211-218, Jan. 2014.

[172] G. Williams and N. McMurray, 'Latent fingermark visualisation using a scanning Kelvin probe', Forensic Sci. Int., vol. 167, no. 2, pp. 102-109, Apr. 2007.

[173] G. Williams, H. N. McMurray and R. C. Newman, 'Surface oxide reduction by hydrogen permeation through iron foil detected using a scanning Kelvin probe', Electrochem. Commun., vol. 27, pp. 144-147, Feb. 2013.

[174] M. Krasemann, H. Streckel, K. Hoffmann, H. Grabke and M. Stratmann, 'Detection of Hydrogen Ingress into Iron Oxide and Iron Oxy-hydroxide Layers by the Kelvin Probe', in Proceedings of the Symposium on Passivity and Its Breakdown, Paris, 1997, vol. 97, p. 207.

[175] S. Evers, C. Senöz and M. Rohwerder, 'Hydrogen detection in metals: a review and introduction of a Kelvin probe approach', Sci. Technol. Adv. Mater., vol. 14, no. 1, p. 14201, Feb. 2013.

[176] S. Evers and M. Rohwerder, "The hydrogen electrode in the "dry": A Kelvin probe approach to measuring hydrogen in metals', Electrochem. Commun., vol. 24, pp. 85-88, Oct. 2012.

[177] S. Modiano et al., 'Changes on iron electrode surface during hydrogen permeation in borate buffer solution', Electrochimica Acta, vol. 53, no. 10, pp. 36703679, Apr. 2008.

[178] T. Casanova and J. Crousier, 'The influence of an oxide layer on hydrogen permeation through steel', Corros. Sci., vol. 38, no. 9, pp. 1535-1544, Sep. 1996. 
[179] A. Bonnel, F. Dabosi, C. Deslouis, M. Duprat, M. Keddam and B. Tribollet, 'Corrosion Study of a Carbon Steel in Neutral Chloride Solutions by Impedance Techniques', J. Electrochem. Soc., vol. 130, no. 4, pp. 753-761, Apr. 1983.

[180] J. Searle, 'Titanium Dioxide Pigment Photocatalysed Degradation of PVC and Plasticised PVC Coatings', University of Wales Swansea, 2002.

[181] H. S. Isaacs, in Advances in Localised Corrosion, U. Bertocci, J. Kruger and S. Smialouzka, Eds. NACE, 1988.

[182] A. M. Simões, A. C. Bastos, M. G. Ferreira, Y. González-García, S. González and R. M. Souto, 'Use of SVET and SECM to study the galvanic corrosion of an iron-zinc cell', Corros. Sci., vol. 49, no. 2, pp. 726-739, Feb. 2007.

[183] G. Song, A. Atrens, X. Wu and B. Zhang, 'Corrosion behaviour of AZ21, AZ501 and AZ91 in sodium chloride', Corros. Sci., vol. 40, no. 10, pp. 1769-1791, Oct. 1998.

[184] G. Song, A. Atrens, D. Stjohn, J. Nairn and Y. Li, 'The electrochemical corrosion of pure magnesium in $1 \mathrm{~N} \mathrm{NaCl}$ ', Corros. Sci., vol. 39, no. 5, pp. 855-875, May 1997.

[185] G. Song, A. Atrens, D. StJohn, X. Wu and J. Nairn, 'The anodic dissolution of magnesium in chloride and sulphate solutions', Corros. Sci., vol. 39, no. 10-11, pp. 1981-2004, Oct. 1997.

[186] J. Elvins, 'The relationship between the microstructure and corrosion resistance of galfan coated steels', Materials Research Centre, Swansea University, 2005.

[187] Stephen Edward. Price, 'Studies of the aluminium inhibition layer in galvanneal / Stephen Edward Price.', University of Wales Swansea, 1999.

[188] Simon O'Dell, 'Investigation into the iron-zinc coating constitution and crater evolution in galvanneal', Thesis - University of Wales Swansea, 2004.

[189] C. Weirman, 'An examination of the corrosion resistance of zinc-magnesium and zinc-aluminium-magnesium coated steels', Materials Research Centre, Swansea University, 2011.

[190] Iwan Davies, 'Development of a processing route for producing galvanised dual phase steel at the Zodiac Galvanising Line / Iwan Davies.', Swansea University, 2010.

[191] S. Hamblyn, 'The Role of the Plate in the Ink Transfer Process in Flexographic Printing', Doctorate of Philosophy, Swansea University, 2004.

[192] 'Lateral-Scanning Interferometry Takes the Long View | Features | Jul 2002 | Photonics Spectra'. [Online]. Available: http://www.photonics.com/Article.aspx?AID=13066. [Accessed: 08-Sep-2016].

[193] S. R. Narayan, G. K. S. Prakash, R. Aniszfeld, A. Manohar, S. Malkhandi and B. Yang, 'High Efficiency Iron Electrode and Additives for Use in Rechargeable IronBased Batteries', US20130149615 A1, 13-Jun-2013.

[194] B. Philip, E. Jewell, P. Greenwood and C. Weirman, 'Material and process optimization screen printing carbon graphite pastes for mass production of heating elements', J. Manuf. Process., vol. 22, pp. 185-191, Apr. 2016. 
[195] Dr Harry Zervos and Dr Bruce Kahn, 'Thin Film Photovoltaics and Batteries 2012-2022'. IDTechEx, 28-Mar-2012.

[196] A. M. Gaikwad, A. C. Arias and D. A. Steingart, 'Recent Progress on Printed Flexible Batteries: Mechanical Challenges, Printing Technologies and Future Prospects', Energy Technol., vol. 3, no. 4, pp. 305-328, Apr. 2015.

[197] D. Agrawal, '12 - Microwave sintering of metal powders', in Advances in Powder Metallurgy, I. Chang and Y. Zhao, Eds. Woodhead Publishing, 2013, pp. 361-379.

[198] F. Zhang, H. Zhang, J. Ren and C. Qu, 'PTFE based composite anion exchange membranes: thermally induced in situ polymerization and direct hydrazine hydrate fuel cell application', J. Mater. Chem., vol. 20, no. 37, p. 8139, 2010.

[199] I. Parenzanovic et al., A mechanistic study of wetting and dewetting during hot dip galvanising of high strength steels. European Commission Technical Steel Research Report, 2006.

[200] I. O. Iwan Davies, 'Method of galvanising a steel strip in a continuous hot dip galvanising line', EP2458022 A1, 30-May-2012.

[201] L. Bordignon et al., 'Enhanced hot dip galvanising by controlled oxidation in the annealing furnace', Eur. Comm. Tech. Steel Res. Rep., no. 20566, pp. 1-177, 2002.

[202] S. Hills, 'Beneficial Effect of Lithiated Electrolyte on Iron Battery Electrodes', J. Electrochem. Soc., vol. 112, no. 10, pp. 1048-1049, Oct. 1965.

[203] G. Williams, 'The use of electrochemical scanning techniques to elucidate corrosion mechanisms in magnesium', presented at the Annual Conference of the Australasian Corrosion Association 2012, 2012, pp. 37-46.

[204] J. H. Sullivan, H. N. McMurray, D. J. Penney and D. A. Worsley, 'Detection of current from a model PIT using the Scanning Vibrating Electrode Technique (SVET)', presented at the ECS Transactions, 2005, vol. 1, pp. 413-423.

[205] G. Williams, H. N. McMurray and R. Grace, 'Inhibition of magnesium localised corrosion in chloride containing electrolyte', Electrochimica Acta, vol. 55, no. 27, pp. 7824-7833, Nov. 2010.

[206] A. C. Bastos, M. G. S. Ferreira and A. M. Simões, 'Comparative electrochemical studies of zinc chromate and zinc phosphate as corrosion inhibitors for zinc', Prog. Org. Coat., vol. 52, no. 4, pp. 339-350, Apr. 2005.

[207] H. N. McMurray, J. R. Searle, B. P. Wilson and D. A. Worsley, 'Investigating changes in corrosion mechanism induced by laser welding galvanised steel specimens using scanning vibrating electrode technique', Br. Corros. J., vol. 37, no. 3, pp. 225230, 2002.

[208] B. P. Wilson, J. R. Searle, K. Yliniemi, T. B. Jones, D. A. Worsley and H. N. McMurray, 'Investigation into the effect of spot weld electrode life and quality on the corrosion behavior of galvanized automotive steel using the three-dimensional scanning vibrating technique', presented at the ECS Transactions, 2012, vol. 50, pp. $53-64$.

[209] B. P. Wilson, J. R. Searle, K. Yliniemi, D. A. Worsley and H. N. McMurray, 'Effect of probe tip inclination on the response of the Scanning Vibrating Electrode 
Technique to an idealised pit-like feature', Electrochimica Acta, vol. 66, pp. 52-60, Apr. 2012.

[210] G. Williams, H. A. L. Dafydd and R. Grace, 'The localised corrosion of Mg alloy AZ31 in chloride containingelectrolyte studied by a scanning vibrating electrode technique', Electrochimica Acta, vol. 109, pp. 489-501, 2013.

[211] H. McMurray, 'The Localised Corrosion Mechanism of Magnesium in Methanol Resolved Using SVET', presented at the 2014 ECS and SMEQ Joint International Meeting (October 5-10, 2014), 2014.

[212] G. Song, 'Recent Progress in Corrosion and Protection of Magnesium Alloys', Adv. Eng. Mater., vol. 7, no. 7, pp. 563-586, 2005.

[213] G. K. Gomma, 'Corrosion inhibition of steel by benzotriazole in sulphuric acid', Mater. Chem. Phys., vol. 55, no. 3, pp. 235-240, Sep. 1998.

[214] P. Matheswaran and A. K. Ramasamy, 'Influence of Benzotriazole on Corrosion Inhibition of Mild Steel in Citric Acid Medium', Journal of Chemistry, 2010. [Online]. Available: https://www.hindawi.com/journals/jchem/2010/598025/abs/. [Accessed: 01-Oct-2017].

[215] L. Hamenu et al., 'Benzotriazole as an electrolyte additive on lithium-ion batteries performance', J. Ind. Eng. Chem., vol. 53, no. Supplement C, pp. 241-246, Sep. 2017.

[216] A. Nazarov and D. Thierry, 'Application of Volta potential mapping to determine metal surface defects', Electrochimica Acta, vol. 52, no. 27, pp. 76897696, Oct. 2007.

[217] G. Williams, N. Birbilis and H. N. McMurray, 'The source of hydrogen evolved from a magnesium anode', Electrochem. Commun., vol. 36, pp. 1-5, Nov. 2013.

[218] C. Gabrielli, G. Maurin, L. Mirkova and H. Perrot, 'Transfer function analysis of hydrogen permeation through a metallic membrane in a Devanathan cell: Part II: Experimental investigation on iron membrane', J. Electroanal. Chem., vol. 590, no. 1, pp. 15-25, May 2006.

[219] H. Masuda, 'SKFM observation of SCC on SUS304 stainless steel', Corros. Sci., vol. 49, no. 1, pp. 120-129, Jan. 2007. 


\section{Appendix A Gwent Electronic Materials Ltd. Iron Inks}

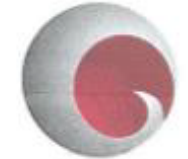

GWENT GROUP

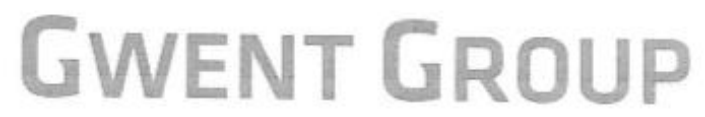

ADVANCED MATERIAL SYSTEMS

\section{CERTIFICATE OF ANALYSIS}

\begin{tabular}{|c|}
\hline C2150413D4 \\
\hline Iron Ink With 10\% PTFE Powder \\
\hline 2150429.07 \\
\hline
\end{tabular}

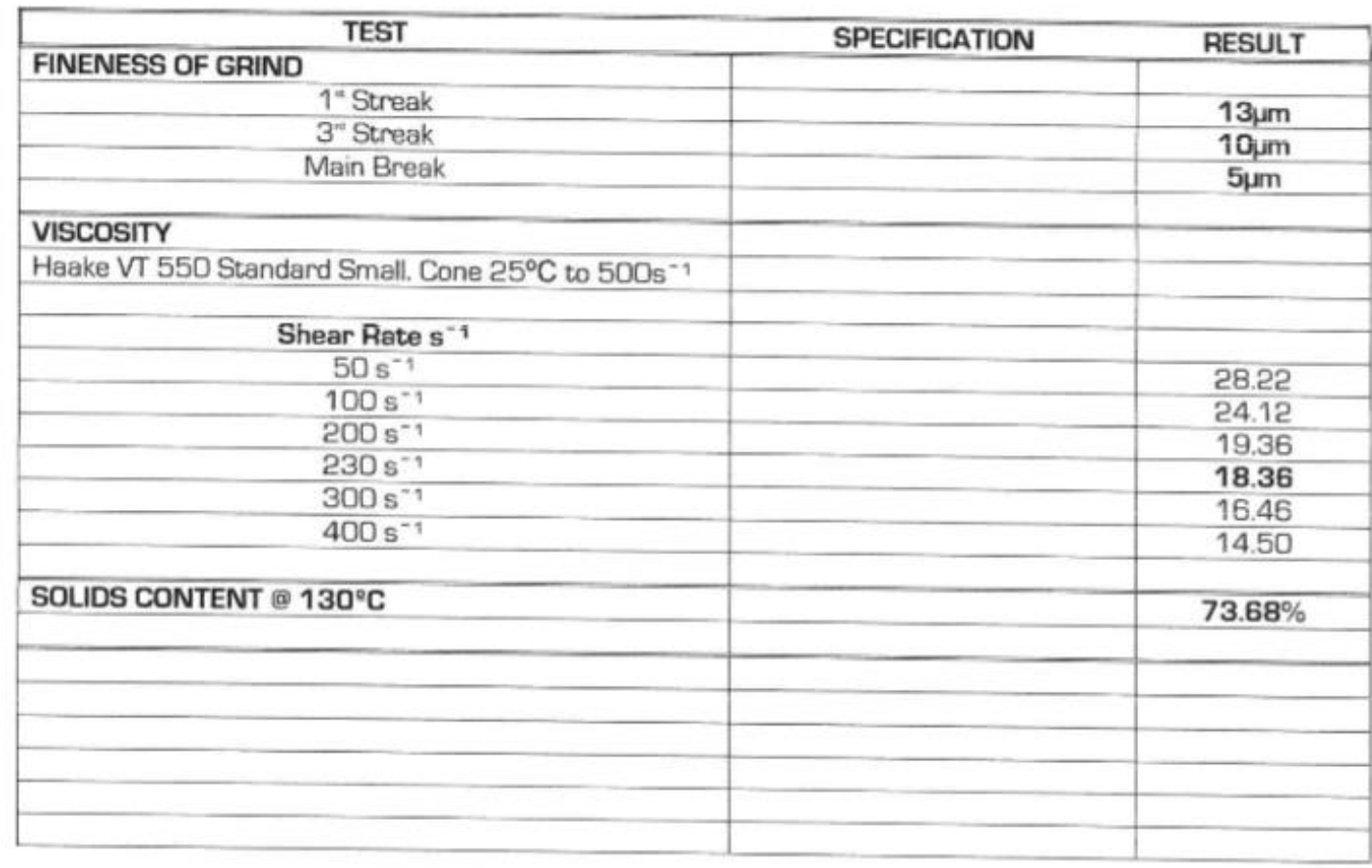

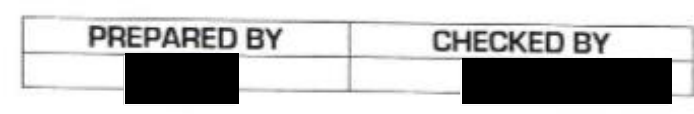

Gwont Electronic Materials Ltd.

Applied Enzyme Technology Lte.

Gwent Biotechnology Systems Lted.

Gwent Sensors itd

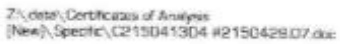

Telephont +44 ing 1905750505

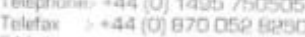

E-Mail quentilquart org

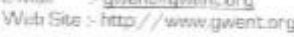

Foge 1 at 1
Monmuvth House.

Mamhiled Park

Pontypoo:

Torfaen.

NP4 OHZ

United Kingdom 


\section{GWENT GROUP}

AdVANCED MATERIAL Systems

GWENT GROUP

CERTIFICATE OF ANALYSIS

\begin{tabular}{|c|}
\hline C2150413D1 \\
\hline Iron Ink \\
\hline 2150429.05 \\
\hline
\end{tabular}

\begin{tabular}{|c|c|c|}
\hline TEST & SPECIFICATION & RESULT \\
\hline \multicolumn{3}{|l|}{ FINENESS OF GRIND } \\
\hline 1 1*Streak & & $11 \mu \mathrm{m}$ \\
\hline $3^{n}$ Streak & & $3 \mu \mathrm{m}$ \\
\hline Main Break & & $1 \mu \mathrm{m}$ \\
\hline \multicolumn{3}{|c|}{ VISCOSITY } \\
\hline \multicolumn{3}{|c|}{ Haake VT 550 Standard Small. Cone $25^{\circ} \mathrm{C}$ to $500 s^{-1}$} \\
\hline \multirow{2}{*}{\multicolumn{3}{|c|}{ Shear Rate s ${ }^{-1}$}} \\
\hline$\frac{\text { Shear Rate s }}{50 \mathrm{~s}^{-1}}$ & & 18.18 \\
\hline$\frac{50 s^{-1}}{100 s^{-1}}$ & & $\begin{array}{l}18.18 \\
16.19\end{array}$ \\
\hline $200 s^{-1}$ & & 13.54 \\
\hline $230 \mathrm{~s}^{-1}$ & & 12.99 \\
\hline $300 s^{-1}$ & & 11.82 \\
\hline $400 \mathrm{~s}^{-1}$ & & 10.70 \\
\hline & & \\
\hline SOLIDS CONTENT @ $130^{\circ} \mathrm{C}$ & & $73.79 \%$ \\
\hline & & \\
\hline & & \\
\hline & & \\
\hline & & \\
\hline & & \\
\hline
\end{tabular}

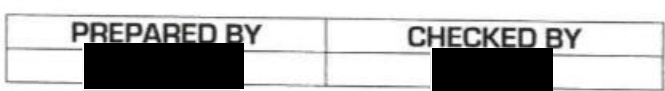

Gwent Electranic Materials Ltd.

Applied Enzyme Technology Ltd

Gwent Biotechnology Systerns Lto

Gwent Sensors Itd.

Telephane -44 (0) 1495750505
Telefax +44 (0) 8700528250

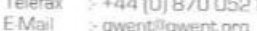

Web Ste hatp

Monmouth House

Mamhilad Park

Pontypool.

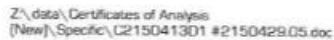

Page 1 \&1

NP4 $\mathrm{OHZ}$

United Kingdom 